\title{
Mixing Processes and Hydraulic Control in a Highly Stratified Estuary
}

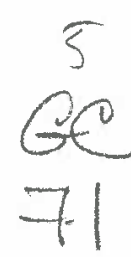

By

\begin{tabular}{|c|c|}
\hline Daniel George MacDonald & MBL / WHOI \\
\hline $\begin{array}{c}\text { B.S.C.E., University of New Hampshire, } 1992 \\
\text { M.S., Cornell University, } 1996\end{array}$ & $\begin{array}{l}\text { LIBRARY } \\
\text { Woods Hole, MA }\end{array}$ \\
\hline Submitted in partial fulfillment of the requirements for the degree of & $\begin{array}{l}\text { Morine Biotogical Laboratory } \\
\text { Woods Hole Oceanographtc } \\
\text { Instrtudian }\end{array}$ \\
\hline
\end{tabular}

at the

MASSACHUSETTS INSTITUTE OF TECHNOLOGY

and the

WOODS HOLE OCEANOGRAPHIC INSTITUTION

February 2003

(C) 2003, Daniel George MacDonald

All rights reserved.

The author hereby grants to MIT and WHOI permission to reproduce paper and electronic copies of this thesis in whole or in part and to distribute them publicly

Signature of Author

$\overline{\text { Joint Program in Oceanography/Applied Ocean Science and Engineering }}$

Massachusetts Institute of Technology and Woods Hole Oceanographic Institution

September 18, 2002

Certified by

Accepted by

W. Rockwell Geyer

Senior Scientist

Thesis Supervisor

Mark A. Grosenbaugh

Chair, Joint Committee for Applied Ocean Science and Engineering

Massachusetts Institute of Technology/

Woods Hole Oceanographic Institution 


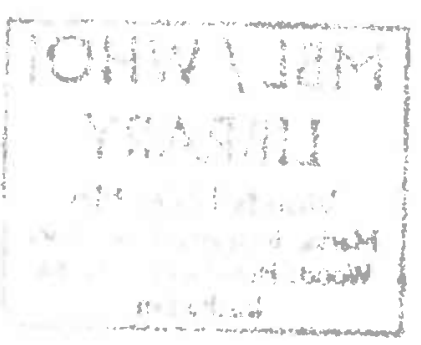




\title{
Mixing Processes and Hydraulic Control in a Highly Stratified Estuary
}

\author{
by
}

\author{
DANIEL G. MACDONALD \\ MIT/WHOI Joint Program in Oceanography and Oceanographic Engineering
}

Submitted to the Massachusetts Institute of Technology/Woods Hole Oceanographic Institution Joint Program in Oceanography and Oceanographic Engineering in September 2002 in partial fulfillment of the requirements for the Degree of Doctor of Philosophy

\begin{abstract}
This thesis utilizes field data from the Fraser River Estuary, a highly stratified system located in southwestern British Columbia, Canada, to investigate the nature of mixing processes in a highly stratified environment, and to extend two-dimensional hydraulic theory to a three dimensional environment.

During the late ebb, a stationary front exists at the Fraser mouth. Although densimetric Froude numbers in the vicinity of the front are supercritical in a frame of reference parallel to the local streamlines, the front itself is oriented such that the value of the Froude number is equal to the critical value of unity when taken in a frame of reference perpendicular to the front. This observation presents a robust extension of established two-dimensional, two-layer hydraulic theory to three dimensions, and implies similarity with trans-sonic flows, in that a Froude angle can be used to identify critical conditions in a manner similar to the Mach angle.

Mixing processes were evaluated at the mouth during the late ebb using a control volume approach to isolate mean vertical entrainment processes from turbulent processes, and quantify the vertical turbulent salt and momentum fluxes. Observed turbulent dissipation rates are high, on the order of $10^{-3} \mathrm{~m}^{2} \mathrm{~s}^{-3}$, with vertical entrainment velocities on the order of $2 \times 10^{-3} \mathrm{~m} \cdot \mathrm{s}^{-1}$. Mixing efficiencies, expressed as flux Richardson numbers, are confined within a range from 0.15 to 0.2 , at gradient Richardson number values between 0.2 and 0.25 . These results are consistent with previous laboratory studies, but represent energetic conditions that are several orders of magnitude higher.

In the estuarine channel, the variability of mixing processes was investigated through the tidal cycle using control volume and overturn scale methods. Spatially, mixing was observed to be more intense near a width constriction on the order of $25 \%$. Temporally, more dominant mixing was observed during ebbs, due to increases in both vertical shear and stratification. Mixing is active and important throughout the tidal cycle, and was found to be the dominant process responsible for removing salt from the estuarine channel during the ebb.
\end{abstract}

Thesis supervisor: Dr. W. Rockwell Geyer

Title: Senior Scientist, Woods Hole Oceanographic Institution 


\section{Acknowledgements}

This work could never have been accomplished without assistance and encouragement from a large number of people. It has truly been a pleasure to work with my advisor, Rocky Geyer, over the last four years. His enthusiasm for his work has been inspiring and I have learned a lot from him. I also appreciate the timely advice and input I have received from the other members of my committee, John Trowbridge, Eric Adams, and Ole Madsen, and I would like to thank Jim Ledwell for chairing my defense.

The Fraser River cruises would not have been possible without help from David Jay, Philip Orton and Doug Wilson. The crew of the R/V Clifford A. Barnes, Ray McQuin and Nikki Hix, was fantastic to work with. Alex Horner was a tremendous help during both cruises, particularly with the collection of the data described in Chapter 4, and its subsequent analysis. Here at WHOI, Terry Donoghue and Jim Irish provided a tremendous amount of assistance in preparation for the two cruises.

The Coastal and Estuarine GFD course at Friday Harbor Labs during the summer of 1999 was a great experience and provided me with a good foundation for the research presented here. I'd like to thank Peter Rhines and Steve Monismith for setting up and running the course, and all the visitors, teaching assistants, and other students for making it such a productive summer. In particular, Jody Klymak and Derek Fong both provided great insight, ideas, and advice as I began to scratch the surface of the Fraser River data.

The other students and staff here at Woods Hole are what make it such a great place to work and study. I'd like to thank the education office staff, Marsha Bissonette, Julia Westwater, and Marcey Simon, for making the administrative side of the Joint Program such a pleasant experience. Other Joint Program students, especially the incoming class of 1998 and all of the AOPE students, have been a great resource for both science and friendship. Many thanks to Charlie Stock, Ben Reeder, Fernanda Hoefel, Joe Warren, Ben Evans, Oscar Pizarro, Jon Woodruff, and many others that have made these years in the Joint Program so worthwhile. Outside of the Joint Program, I have to thank Eric Amundsen for convincing me to head back to graduate school, and Paul Arnold, Dave Corso, and Bruce Eaton for many great hikes over the years.

My most consistent supporters on the long road towards this $\mathrm{Ph} . \mathrm{D}$. have always been my family: my parents, Ken and Claire, and my brothers, Rob and Jon. Their encouragement over the past 32 years has been irreplaceable. My wife Lisa has always provided me with unqualified support in my graduate school and other endeavors since we first met. Her support has been limitless, and so is my appreciation. Thank you Lisa.

This research was funded by Office of Naval Research grants N000-14-97-10134 and N000-14-97-10566, National Science Foundation grant OCE-9906787, a National Science Foundation graduate fellowship, and the WHOI Academic Programs Office. 


\section{Contents}

$\begin{array}{ll}\text { Abstract } & 3\end{array}$

Acknowledgements $\quad 5$

1 Introduction $\quad 15$

1.1 The Physical Significance of Estuaries................................................... 16

1.2 The Physics of Mixing in Stratified Fluids ............................................. 18

1.3 Two-Layer Hydraulic Theory ............................................................... 21

1.4 The Fraser River Estuary .................................................................... 24

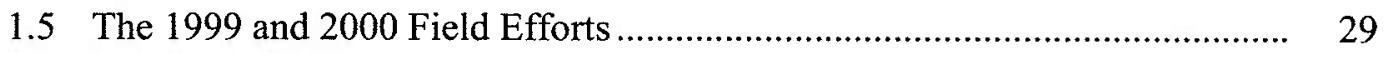

1.6 Structure of the Thesis ......................................................................... 31

2 The Three-Dimensional Structure and Hydraulic Control of a Highly 35 Stratified Estuarine Front

$2.1 \quad$ The Near-Field Plume ....................................................................... 37

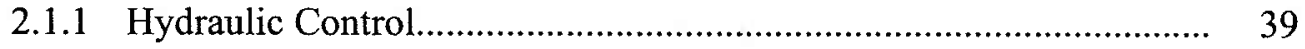

2.1.2 Structure of River Plumes .......................................................... 40

2.2 The Fraser Estuary and the 2000 Field Effort ........................................... 43

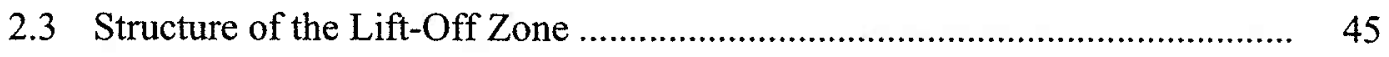

2.3.1 Streamlines of the Upper Layer ................................................... 46

2.3.2 Lift-Off Cross Sections ............................................................... 48

2.3.3 Front Location and Temporal Variability ..................................... 51

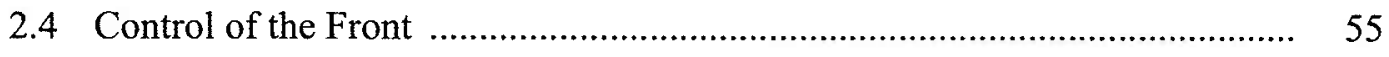

2.4.1 Interface Height Determination ............................................. 58 
2.4.2 Froude Angle .............................................................................. 60

2.4.3 Expansion Control vs. Bottom Control ........................................... 64

2.5 Kinematics of the Salt Supply .................................................................. 66

2.5.1 Distribution of Salt Flux in Salinity and Vertical Space ................... $\quad 70$

2.5.2 Potential Energy Changes Within the Lift-Off Zone ........................ 75

2.6 Cross Channel Dynamics ............................................................................... $\quad 77$

2.7 Implications of Froude Angle Control and Local Kinematics ....................... 81

2.7.1 Importance of Lateral Flow .............................................................. 85

2.8 Concluding Remarks on Lift-Off Zone Structure ……............................... 86

3 Turbulent Energy Production and Entrainment at a Highly Stratified 89 Estuarine Front

3.1 Mixing in Shear-Stratified Flows _........................................................... 91

3.1.1 Turbulent Kinetic Energy and Mixing Efficiency ……...................... 91

3.1.2 Entrainment Velocity .................................................................... 95

3.1.3 Turbulent Length Scales ….......................................................... 100

3.2 The Fraser River and the 1999 Field Effort ……...................................... 102

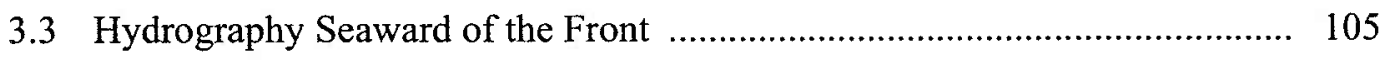

3.4 Estimation of Turbulent Fluxes ……...................................................... 109

3.4.1 Measured Quantities ..................................................................... 113

3.4.2 Volume Conservation and Vertical Velocity ................................... 117

3.4.3 Salt Conservation ...................................................................... 119

3.4.4 Momentum Conservation ……………………............................. 121

3.4.5 Flux Richardson Numbers ......................................................... 126

3.5 Lateral Effects …........................................................................... 128

3.6 Turbulence, Entrainment and Closure ………........................................ 133

3.6.1 Local Production vs. Advected TKE _............................................ 134

3.6.2 Mean Vertical Velocities .............................................................. 136 
3.6.3 Mechanisms of turbulence generation ...................................... 137

3.7 Concluding Remarks on Mixing in the Lift-Off Zone ............................... 139

4 The Variability of Vertical Salt Flux in a Highly Stratified Estuarine 141 Channel

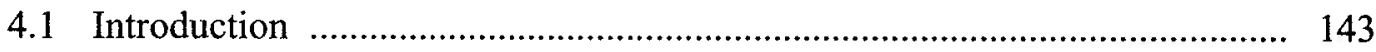

4.2 The Mechanics of Shear-Induced Mixing …............................................. 146

4.2.1 Gradient Richardson Number ............................................... 146

4.2.2 Froude Numbers and Hydraulic Theory ..................................... 147

4.2.3 Tubulent Salt Transport and Buoyancy Flux ............................... 147

4.2.4 Overturn Scales ................................................................ 148

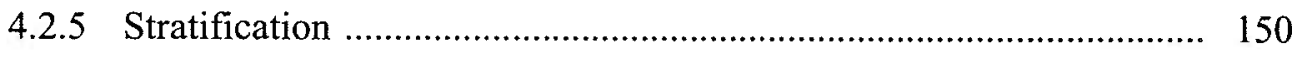

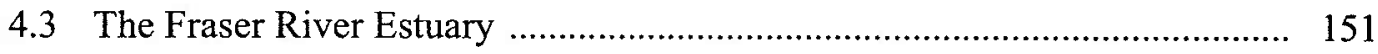

4.4 Data Collection During Freshet, Spring Tide Conditions .......................... 155

4.4.1 Integrated Observations From a Down-Stream Time Series ............ 155

4.4.2 Buoyancy Flux Estimates Using a Control Volume Approach ......... 160

4.4.3 Overturn Scale Estimates ....................................................... 166

4.4.4 Stratification Profiles ................................................................... 170

4.4.5 Richardson Number Profiles ....................................................... 173

4.4.6 Froude Number Profiles .......................................................... 178

4.5 Temporal Variability of Mixing Processes f............................................... 181

4.5.1 Initial Flood ....................................................................... 181

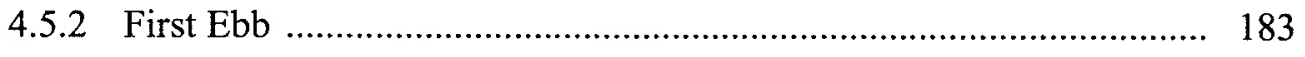

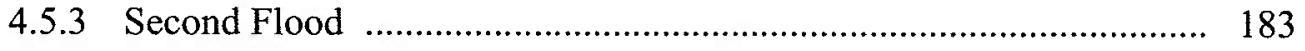

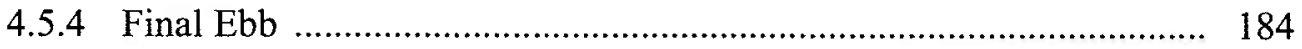

4.6 Spatial Variability of Mixing Processes During Ebb Tide ......................... 185

4.7 A Summary of Mixing Through the Tidal Cycle .................................... 186

4.7.1 Mixing in the Fraser ............................................................. 186 
4.7.2 Comparisons With Other Estuaries ................................................ 191

4.8 Concluding Remarks on Mixing Variability …………................................ 192

5 Conclusions 193

5.1 The Dynamic Cycle of the Fraser River ………………............................. 194

5.2 Contributions of the Thesis _................................................................. 197

5.2.1 Three-Dimensional Extension of Two-Layer Hydraulic Theory ....... 197

5.2.2 Development of Control Volume Method For Turbulent Flux

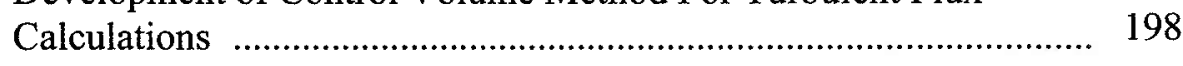

5.2.3 Mixing Efficiencies $\left(R i_{f}\right)$ in Energetic Shear-Stratified Flows ......... 199

5.2.4 Simple Turbulent Closure Scheme for Pure Shear-Stratified Flows .. 199

5.2.5 Importance of Mixing Throughout the Tidal Cycle .......................... 200

5.3 Unresolved Issues ................................................................................... 201

Appendix: A New Stratification Parameter 203

$\begin{array}{ll}\text { Bibliography } & 207\end{array}$ 


\section{List of Figures}

1.1 Location and plan view of the Fraser River Estuary f.................................... 25

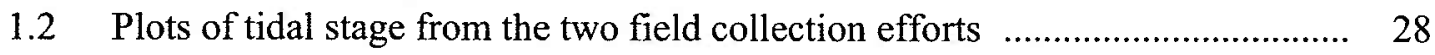

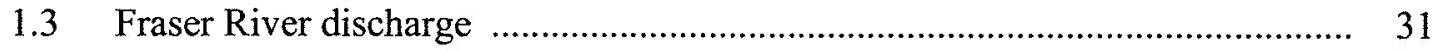

2.1 Definition sketch for front and near-field regions ….................................. 38

2.2 Estuarine channel, and mouth regions of the Fraser River Estuary ................... 44

2.3 Mouth of the Fraser, with upper layer streamlines ....................................... 47

2.4 Along channel cross-sections through the lift-off zone ................................. 49

2.5 Transverse channel cross-sections through the lift-off zone .......................... 50

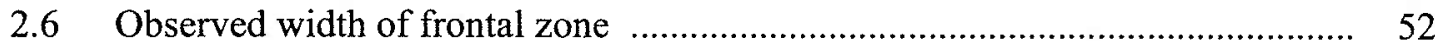

2.7 Maps of the intersection of the $20 \mathrm{psu}$ isohaline with the bottom .................... 54

2.8 Upper layer Froude number across the lift-off zone ..................................... 57

2.9 Streamwise cross section, velocity profiles, and interface height $\ldots . . . \ldots \ldots \ldots \ldots \ldots . . . . . . . .59$

2.10 Propogation of wave fronts for critical and supercritical flow ....................... 61

2.11 Froude angle estimates for lift-off region ................................................. 63

2.12 Froude Angle Path and Prandtl Meyer Expansion Fan at Sand Heads ............. 65

2.13 Zones for control volume salt balance analysis ......................................... 67

2.14 Definition sketch for control-volume salt balance analysis .......................... 68

2.15 Distribution of salt flux relative to salinity ............................................... 71

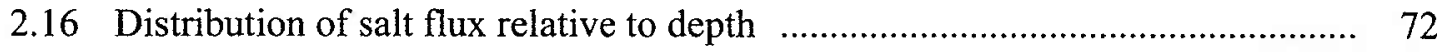

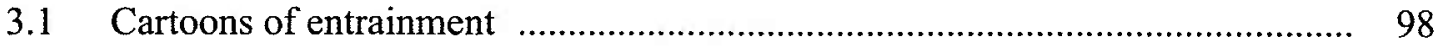

3.2 Plan view of estuary mouth, with ship tracks ......................................... 104

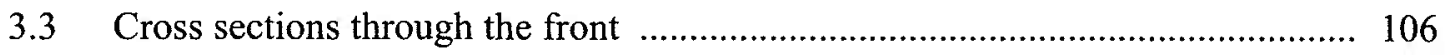

3.4 Profiles of the squared composite Froude number across the front ................. 110

3.5 Two-dimensional schematic of the control volume method ............................. 114 
3.6 Vertical profiles of velocity and salinity in the control volume region ............. 115

3.7 Vertical profiles of the gradient Richardson number .................................... 116

3.8 Vertical profiles of the mean vertical velocities, $w_{h}$ and $w_{j} \ldots \ldots \ldots \ldots \ldots \ldots \ldots \ldots . . . . . . . . . . .118$

3.9 Buoyancy flux, and eddy diffusivity profiles from control volume analysis ... 120

3.10 Estimates of surface elevation across the lift-off region ................................. 122

3.11 Turbulent momentum flux profiles from control volume analysis .................. 124

3.12 TKE production, and eddy viscosity profiles from control volume analyses .... 125

3.13 Flux Richardson number as a function of the gradient Richardson number ...... 127

3.14 Profiles of cross-stream velocity and lateral volume flux ratio ........................ 130

3.15 Lateral influx ratios for both salt and momentum ....................................... 132

4.1 The main arm of the Fraser River ............................................................ 153

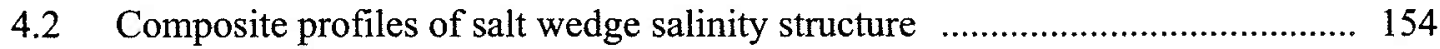

4.3 Tidal height, surface velocity, and bottom velocity at the anchor station ......... 156

4.4 Contours of salinity through a tidal cycle at the anchor station $\ldots \ldots \ldots \ldots \ldots \ldots \ldots \ldots . . . . . . . .157$

4.5 Integrated landward and seaward salt fluxes at anchor station $\ldots . \ldots \ldots \ldots \ldots \ldots \ldots \ldots . . . . . . . .159$

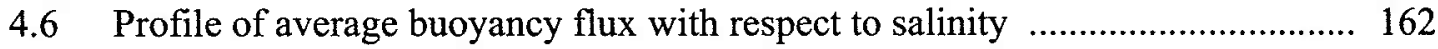

4.7 Spatial variability of buoyancy flux during the ebb (control volume analyses). 165

4.8 Temporal variability of overturn scale and buoyancy flux at anchor station .... 167

4.9 Salinity profile at the anchor station, two hours after second high tide ............ 169

4.10 Spatial variability of overturn scale and buoyancy flux during ebb .................. 171

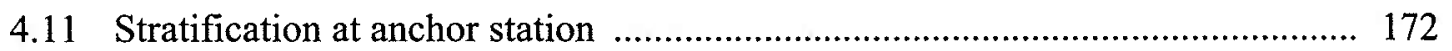

4.12 Subcritical Richardson numbers through the tidal cycle at anchor station ....... 174

4.13 Bulk Richardson number through the tidal cycle at anchor station ….............. 176

4.14 Subcritical Richardson numbers across the channel during ebb ...................... 177

4.15 Froude number time series at anchor station ............................................. 179

4.16 Froude number profiles during ebb ....................................................... 180

4.17 Temperature salinity relationships for time series CTD casts ........................ 182

4.18 Schematic representation of boundary mixing due to secondary circulation $\ldots . .187$ 


\section{List of Tables}

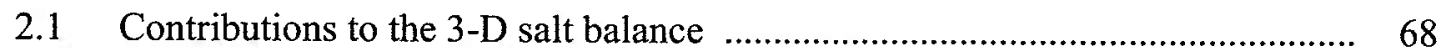

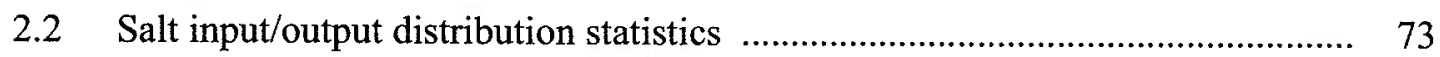

4.1 Summary of buoyancy flux estimates through the tidal cycle ....................... 190 


\section{Chapter 1}

\section{Introduction}




\subsection{The Physical Significance of Estuaries}

The discharge of fresh river water represents a significant source of nutrients, sediments, pollutants and other terrestrially derived material to the world's oceans (Yin et al., 1995; Caspers, 1967; Sommerfield and Nittrouer, 1999). During its journey to the sea, the fresh river discharge first interacts with ocean water within an estuary situated at or near the river mouth. Because very different water masses are colliding within an estuary, estuaries can often be characterized by strong gradients, of which the most dynamically important are density and velocity. Strong gradients of dissolved and suspended constituents such as nutrients, pollutants, and suspended sediment are also typically present, and it is the ultimate disposition of these components that provides much of the motivation for understanding the local physics.

The nature and intensity of the mixing processes within an estuary are driven, to a large extent, by the dynamically important gradients of density and velocity. Shear instabilities resulting from perturbations in the velocity profile large enough to overcome the ambient density stratification provide the primary mechanism for turbulence generation and mixing (Thorpe, 1973). At the seaward end of the estuary, the density difference between the discharge and the ambient coastal water has been greatly reduced, but the brackish residual that remains can continue to persist in the coastal ocean and be carried great distances, sometimes on the order of $10^{2}$ to $10^{3}$ kilometers, as the plume forms a coastal current (Yankovsky and Chapman, 1997). Eventually, through the action of external forcing mechanisms, such as wind and wave action, the signal of the plume is diluted by mixing with ocean water (Fong, 1998; Lentz, 2001). 
The physics of the estuarine region are responsible for setting the initial conditions of the coastal current, ultimately affecting far-field transport and the dilution and distribution of fresh water and river borne material. The use of numerical models to understand and predict the physics of coastal currents is promising, however an important boundary condition for these numerical models must represent the conditions at the river mouth, which can only be assessed through an understanding of the local physics. Specifically, initial values of plume velocity, width, depth, and density are important parameters that represent the integrated product of smaller scale physical processes within the estuary. It is these values that would need to be passed to a numerical model as boundary conditions for a coastal current. Accurate prediction of these parameters has been a goal of estuarine research for at least fifty years (e.g., Stommel and Farmer, 1952), although a satisfactory predictive capacity has yet to be established.

This thesis focuses on field observations from the Fraser River Estuary, a highly stratified estuary in southwestern British Columbia, Canada, in an effort to understand both the physics of mixing processes in highly stratified environments, and the factors controlling the hydrographic structure of an estuary, particularly the estuarine front. The thesis addresses specific mixing and hydraulic control processes which are critical for fixing coastal current boundary conditions.

A second motivating factor for the present study is the relevance of the general principles of fluid dynamics observable in estuaries to a broader range of both basic and applied problems. Similar flows and fluid dynamics are important in a wide range of fields that includes oceanography, meteorology, and engineering. The physics of 
turbulent regions characterized by both velocity shear and stratification has been studied for several decades, primarily through laboratory experiments, numerical models, and oceanic thermocline observations (e.g., Ivey and Imberger, 1991; Smyth and Moum, 2000; Gregg, 1989). The present study complements the current body of knowledge by adding observations from a highly energetic stratified shear layer. Likewise, the present study provides a three-dimensional extension to the concept of two-layer hydraulic control, which has been well studied analytically in one dimension (Armi, 1986; Dalziel, 1991).

\subsection{The Physics of Mixing in Stratified Fluids}

Velocity shear in a fluid of constant density provides a source for instability and the generation of turbulence. Stable stratification within a fluid has the opposite effect, providing a stabilizing influence to the flow. In a stratified shear flow, both of these mechanisms are important and a balance is struck between the two. The nature of the balance can be quantified by the gradient Richardson number, $R i_{g}$, equal to the ratio of the square of the buoyancy frequency to the square of the vertical velocity shear:

$$
R i=\frac{-\frac{g}{\rho} \frac{\partial \rho}{\partial z}}{\left(\frac{\partial u}{\partial z}\right)^{2}}
$$

where $g$ is gravitational acceleration, $\rho$ is density, $u$ is velocity, and $z$ is the vertical coordinate. Miles (1961) and Howard (1961) demonstrated analytically that at values of $R i_{g}>1 / 4$ the density gradient is strong enough to suppress the development of turbulence, 
and that a value of $R i_{g}<1 / 4$ was a necessary, but not sufficient, condition for the generation of turbulence. Thorpe (1973) supplemented this work by demonstrating experimentally that a value of $R i_{g}$ equal to $1 / 4$ was a good indicator for the onset of stratified turbulence, and establishing this threshold as a sufficient condition for turbulence in a laboratory shear flow.

More recent work, over the last few decades, has focused on quantifying rates of turbulent kinetic energy (TKE) production and dissipation in shear stratified environments in an effort to understand both the conversion of mean flow energy into turbulence, and the ultimate partitioning of TKE to potential energy, in the form of buoyancy flux, and dissipation (e.g., Ivey and Imberger, 1991). Most of this work has been performed in the laboratory (e.g., Linden, 1979; Ivey and Imberger, 1991), or is based on observations from the deep ocean thermocline (e.g., Gregg, 1987). This existing body of knowledge suggests that the flux Richardson number, $R i_{f}$, which is a measure of mixing efficiency equal to the ratio of buoyancy flux to TKE production, should be approximately constant for fully developed shear-stratified turbulence at a value between 0.15 and 0.25 . Most of the data upon which this conclusion is based are from systems with relatively low dissipation rates and small mean flow and turbulent scales, which may not be representative of actual environmental flows.

One scaling measure that can be used to describe stratified turbulence is a Reynolds number constructed from turbulent scales (Stillenger et al, 1982):

$$
\operatorname{Re}_{r}=\frac{q L_{o}}{v}=\frac{\varepsilon}{v N^{2}}
$$


where $q$ and $L_{o}$ are characteristic velocity and length scales of the turbulence, $v$ is the molecular viscosity of the fluid, $\varepsilon$ represents dissipation of turbulent kinetic energy, and

$$
N=\left(-\frac{g}{\rho} \frac{\partial \rho}{\partial z}\right)^{\frac{1}{2}}
$$

is the buoyancy frequency. The value of this turbulent Reynolds number is limited in the laboratory by the limited length scales associated with laboratory apparatus, and, as a result, field scale values cannot be approximated in the laboratory. At values of this Reynold's number that are too small, molecular diffusion effects for salt and/or heat can begin to impact the mixing processes. Another potential drawback of laboratory work is that laboratory studies are often confined to two dimensions, removing the effects of secondary flows from the analysis. Values of $R e_{T}$ for laboratory flows typically fall below $10^{2}$, whereas naturally occurring environmental flows are often characterized by values several orders of magnitude higher. Little is known about the mechanics of shearstratified turbulence at these scales, particularly the relationship of TKE production to the mean flow, and the nature of the flux Richardson number.

TKE quantities are typically measured in the field using microstructure profilers, which have sufficiently high resolution to measure turbulent fluctuations effectively (e.g., Gargett and Moum, 1995; Peters, 1999). An alternative technique for directly measuring TKE production involves analyzing the variance associated with measurements from a stably positioned acoustic Doppler current profiler (ADCP), as described by Stacey et al. (1999). This technique can be effective when there is minimal ambient motion of the ADCP unit. An indirect measurement of buoyancy flux can also be achieved through the 
use of overturn scale methods (e.g., Thorpe, 1979; Osborn, 1980; Dillon, 1982), using simple observations of density profiles. Although these methods can all be effective, each suffers from constraints imposed by cost, implementation, and/or the necessity of assumptions related to the turbulent processes that are the subject of the investigation. No direct measurements of TKE quantities have been published that utilize velocity and density profiles derived from ADCP and conductivity-temperature-depth (CTD) measurements. These instruments are widely available and are two of the most common pieces of instrumentation for modern observational physical oceanography. Therefore, a method utilizing this type of data could be a useful alternative to the three methods mentioned above for identifying TKE quantities under certain conditions.

\subsection{Two-Layer Hydraulic Theory}

The understanding of stratified flows has advanced substantially in the past 50 years. Early studies focused on the large-scale advective effects associated with the motion of varying density fluids such as fresh and salt water, often referred to as the "lock exchange problem", (Schijf and Schonfeld, 1953). Additional work has focused more specifically on issues of hydraulic control in these types of flows. An internal Froude number, $G$, for a two-layer flow can be defined as the square root of the sum of the squares of the individual layer Froude numbers (e.g., Armi, 1986):

$$
G^{2}=F_{1}^{2}+F_{2}^{2}, \quad F_{j}^{2}=\frac{u_{j}^{2}}{g^{\prime} h_{j}},
$$


where the subscripts 1 and 2 refer to the upper and lower layers, respectively, $F_{j}$ is the layer Froude number, $h_{j}$ is the layer thickness, and $g^{\prime}=g\left(\rho_{1}-\rho_{2}\right) \rho_{\circ}^{-1}$ is a reduced gravity. As early as 1952, Stommel and Farmer first suggested that a critical Froude number value equal to one was a necessary condition at an estuarine salt wedge front. Several decades later, hydraulic theory for two-layer flows was developed through several different analytical methods (Armi, 1986; Armi and Farmer, 1986; Farmer and Armi, 1986; Dalziel, 1991). Two-layer hydraulic theory can be considered an extension of classical hydraulic theory for uniform density open channel flow. In a manner analogous to traditional hydraulics, the internal Froude number can be used to describe the state of the flow with respect to the communication of information, in this case via long interfacial waves, in both the upstream and downstream directions.

A value of $G<1$ is representative of subcritical flow, indicating that the ambient fluid velocity is less than the wave speed, which allows waves to propagate in both directions. Supercritical flow is achieved when $\mathrm{G}>1$, indicating that both the upstream and downstream directed wave fronts are swept downstream by the mean current, preventing information from propagating in the upstream direction. Hydraulic control can be established at transition points in the flow, where Froude numbers are forced to a critical value equal to 1 , establishing a consistent relationship between discharge and interfacial depth. A common example of hydraulic control in a uniform density open channel flow is that of flow over a weir (e.g., Henderson, 1966, pp. 174-234). This phenomenon has been long exploited as an efficient means of measuring flow rates. 
Armi and Farmer (1986) and Farmer and Armi (1986) presented classification schemes for the hydraulic control of two-layer flows through contractions and over sills, respectively, based on barotropic flow components and the internal, or individual layer, Froude numbers. The changes in channel geometry due to the sill and/or contraction result in a convective acceleration of the fluid, with significant changes in Froude number. The flow is then controlled at a specific point, depending on the barotropic component of the flow, where the local velocity and depth are matched to result in a critical Froude number of unity. According to Armi and Farmer (1986) and Farmer and Armi (1986), a point of hydraulic control is located at an arrested density front, with supercritical conditions behind the front.

The relevance of two-layer hydraulic theory to observations of estuarine fronts has been approached in several previous studies of highly stratified estuaries, including the Mississippi River Estuary (Wright and Coleman, 1971), and the Ishikari River Estuary (Kashawamura and Yoshida, 1978). These observations have indicated that the Froude number is close to one near observed fronts. Observations of the Mississippi River plume indicate that vertical mixing was probably intensified as a result of an observed increase in Froude number to marginally supercritical conditions near the river mouth. The Froude number was observed to decrease within the plume, outside of the mouth, to marginally subcritical values, suggesting a possible feedback mechanism with a natural tendency towards criticality. Kashawamura and Yoshida (1978) found a similar distribution of Froude number associated with the Ishikari River in Japan. Subcritical flow was found inside the river channel, with approximately critical conditions at the 
mouth, but a decrease of Froude number outside the mouth was not consistently observed.

The studies of Wright and Coleman (1971) and Kashawamura and Yoshida (1978) used observations of approximately critical Froude numbers near estuarine fronts to validate the existing two-dimensional theory (i.e., $G=1$ at points of hydraulic control). These conclusions were based in part on historical limitations in measurement equipment, and a limited number of observation points, which made it difficult to determine the nature of hydraulic control in these estuaries more precisely. Other fronts (i.e., Geyer et al., 1988) have been observed in estuaries at significantly supercritical values, which are inconsistent with existing two-layer hydraulic theory. Although a three-dimensional approach has been undertaken for the description of hydraulic control in unstratified open channel flows (Ippen, 1936) and similar controls in trans-sonic gas flows (Garvine, 1982), a similar approach has not been used to expand the scope of two-layer hydraulic theory. With the advent of modern oceanographic instruments, specifically the ADCP and CTD, previous observational deficiencies can be overcome, and much needed observations of frontal structure can be made in order to develop and test a threedimensional extension of classic two-layer hydraulic theory.

\subsection{The Fraser River Estuary}

The Fraser River Estuary is located at the mouth of the Fraser River, which flows into the Strait of Georgia in the southwestern corner of British Columbia, approximately $20 \mathrm{~km}$ south of the City of Vancouver (Figure 1.1). With a total length of $1,368 \mathrm{~km}$, the 

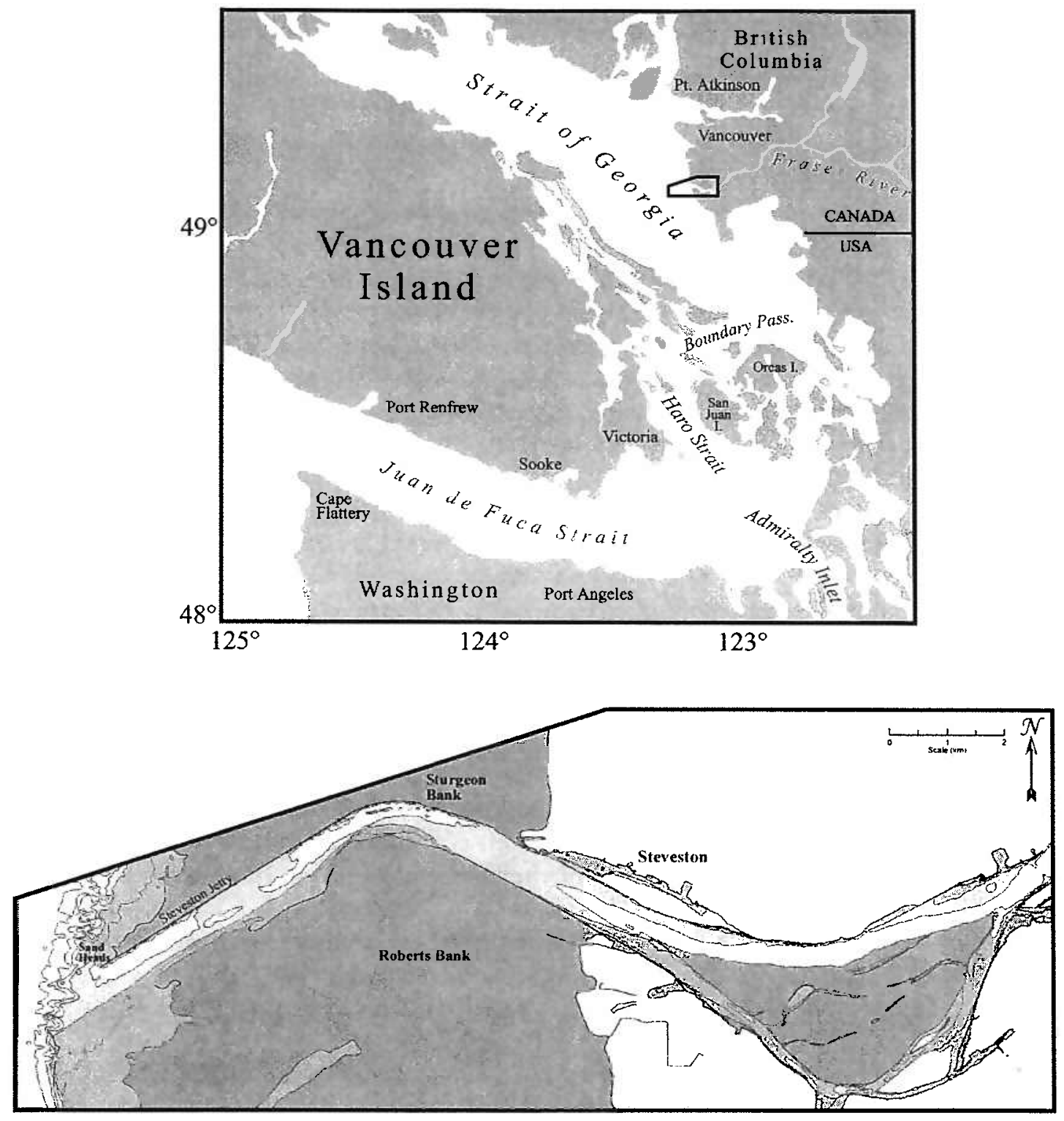

Figure 1.1: Location and plan view of the Fraser River Estuary, British Columbia, Canada. The second panel shows the lower $20 \mathrm{~km}$ of the main arm of the Fraser River and its confluence with the Strait of Georgia. Darkest shading represents banks that are submerged at high water. Remaining shading represents depths of 0-5 m, 5-10 m and greater than $10 \mathrm{~m}$ from dark to light. Depths within the main channel generally range from 10 to $15 \mathrm{~m}$, with depths seaward of Sand Heads dropping rapidly to greater than $50 \mathrm{~m}$. 
Fraser River is the longest river in British Columbia, and together with the Columbia and Peace Rivers, drains the majority of the western slopes of the Canadian Rockies and the Coast Range mountains. The watershed of the Fraser encompasses $234,000 \mathrm{~km}^{2}$, making the Fraser the dominant source of freshwater to the inland sea created by the Strait of Georgia, the Strait of Juan de Fuca, and Puget Sound. The freshet in the Fraser typically occurs in late May or early June as snow pack at the higher elevations of the inland mountains begins to melt. Peak freshwater discharge during the freshet is on the order of 10,000 cubic meters per second.

This study focuses on the dynamics of the Fraser River Estuary during the summer freshet, which is highly stratified through the majority of the tidal cycle. High stratification is maintained by the high fresh water discharge. In addition, the estuary is also affected by strong tides, potentially leading to more energetic mixing than other highly stratified, or salt wedge, estuaries. The conditions in the Fraser are well suited for observations of general fluid mechanics phenomena in a high energy, field scale environment. The flow in a highly stratified system such as the Fraser is more similar to an idealized two-layer flow than the circulation patterns observed in other types of estuaries (i.e., well-mixed, or partially mixed), which makes the Fraser suitable for observations of both the mixing processes along a highly stratified interface, and the structure and position of the stratified front with respect to two-layer hydraulic theory.

Bathymetry within the estuary is relatively uniform due to maintenance of the channel for navigation. The main stem of the channel, which is dredged regularly and confined by jetties, varies from 600 to $1,000 \mathrm{~m}$ in breadth, with a fairly uniform depth of 
approximately 10 to $15 \mathrm{~m}$. Depths increase rapidly to over $50 \mathrm{~m}$ outside the river mouth in the Strait of Georgia. The lower $12 \mathrm{~km}$ of the river channel is predominately straight, with the notable exception of Steveston Bend, a bend of roughly $60^{\circ}$ located approximately $6 \mathrm{~km}$ landward of the mouth.

The combination of high discharge and large tidal amplitude in the Fraser River Estuary creates a highly energetic system at the river mouth. Large amplitudes, typically ranging from 2.5 to 4.0 meters, and a pronounced diurnal component characterize tides in this area, as shown in Figure 1.2. The resulting salt wedge typically intrudes some 10 to $20 \mathrm{~km}$ landward of Sand Heads, which represents the seaward end of the Steveston Jetty, on each tidal cycle, retreating to the mouth during the larger of the two daily ebbs. The front then remains nearly stationary at the mouth for several hours prior to reentering the channel on the following flood.

The stratification in the Fraser River is controlled almost exclusively by salinity. Although temperature variations on the order of $6^{\circ} \mathrm{C}$ can be observed, local salinity differences on the order of 20 to 30 psu dominate changes in density. This simplifies calculations, as isopycnal surfaces can be assumed to be coincident with isohalines

The work of Geyer and Smith (1987), Geyer (1988), and Geyer and Farmer (1989) in the Fraser River Estuary focused on the time-dependent variation of the salt wedge across the tidal cycle. The salt wedge was observed to advance landward during the flood as a density current, with little mixing along the density interface. The shape of the intrusion changed significantly on the ebb, as the structure was eroded by shear instabilities, primarily Kelvin-Helmholtz and Holmboe instabilities, along the interface. The 
(a)

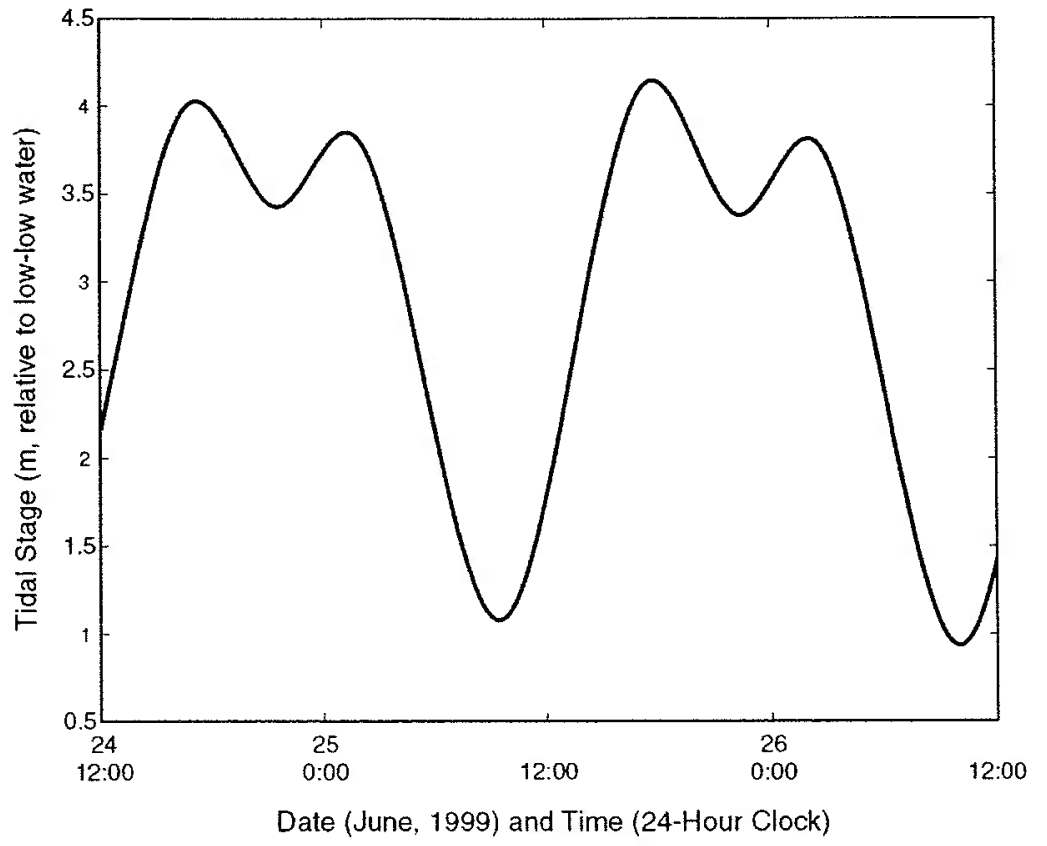

(b)

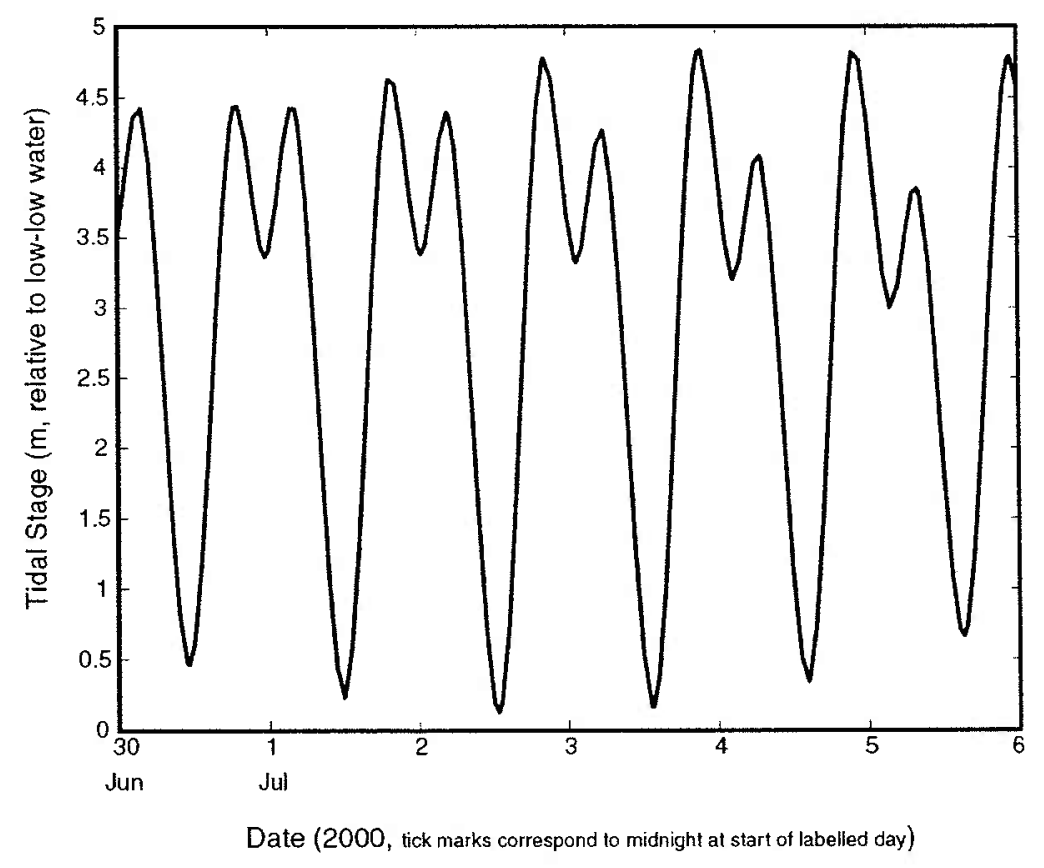

Figure 1.2: Plots of tidal stage from the two field collection efforts. (a) 1999. (b) 2000. Local time is shown, Pacific Daylight Savings Time (PDST). 
difference between these two states was attributed to a transition of the internal hydraulic state of the flow. On the flood, the advancing current was characterized by subcritical densimetric Froude numbers, but during the ebb, a transition towards a supercritical state was observed. It was hypothesized that more intense mixing was associated with the supercritical regions, which were typically found at and seaward of localized narrows in the channel.

Although these studies (Geyer, 1985; Geyer and Smith, 1987; Geyer, 1988, Geyer and Farmer, 1989) provide a solid framework for understanding the dynamics of the Fraser River salt wedge through the tidal cycle, quantitative measurements of turbulence and mixing could not be made. Advances in instrumentation over the last 15 years have made such measurements more accessible. Estimates of buoyancy flux through the tidal cycle from observations during the current study, with comparisons to the earlier conclusions, are presented in Chapter 4.

\subsection{The 1999 and 2000 Field Efforts}

This thesis is based on observations from two field efforts, during the summer freshets of 1999 and 2000. Both field efforts involved the collection of hydrographic data from shipboard instruments, using the UNOLS vessel R/V Clifford A. Barnes out of the University of Washington. Data was collected primarily from two hull-mounted Acoustic Doppler Current Profilers (ADCPs), and a towed Ocean Sensors 200 Series conductivity-temperature-depth (CTD) unit. Combined use of two ADCPs, at $1200 \mathrm{kHz}$ 
and $300 \mathrm{kHz}$, enabled consistent bottom tracking coupled with a vertical resolution on the order of $25 \mathrm{~cm}$ across the top $25 \mathrm{~m}$ of the water column.

Data collection at the mouth during the 1999 field study consisted of repeated ship tracks across the front during the late ebb portion of the tidal cycle. In 2000 , parallel tracks were used, in order to develop a three-dimensional picture of the frontal structure. Data from an anchor station time series, and transects within the lower $10 \mathrm{~km}$ of the estuarine channel during the ebb are also incorporated into the present study.

The tidal range during the course of both field efforts was on the order of $4 \mathrm{~m}$, as shown in Figure 1.2. All of the data from the 1999 effort incorporated into the present study was collected on July 25 , five days past neap tide. Data collection during the 2000 freshet was conducted between June 30 and July 4, and was centered around the spring tide, which occurred on July $2^{\text {nd }}$, with a tidal amplitude of approximately $4.25 \mathrm{~m}$.

The freshet of 1999 was unusually large, and it occurred later in the year than usual. River discharge on July $25^{\text {th }}$ was approximately 7,500 cubic meters per second (cms), as measured at the most seaward gauging station, located at Hope, approximately $150 \mathrm{~km}$ upstream of the mouth. The peak discharge in 1999 occurred in late June and was on the order of $11,000 \mathrm{cms}$, compared with an average peak on the order of 7,000 cms. This was the result of record-breaking snowfall in the watershed during the winter of 1998-99, followed by cold spring and summer weather. The resulting freshet lasted more than six weeks from late June into the beginning of August. The extended length of the freshet averted the significantly higher discharges and potentially disastrous flooding that would have occurred under more typical, warmer conditions, and provided a longer period for 
observations. The 1999 discharge stands in contrast to the more typical discharge observed during the 2000 freshet. Discharge during the 2000 field effort increased steadily from approximately 6,500 to $7,200 \mathrm{cms}$, as measured at Hope, over the five day cruise. Discharge curves for both 1999 and 2000 are compared with an 85-year mean in Figure 1.3.

Despite significant differences between the overall discharge curves between 1999 and 2000 , hydrologic conditions during the two sampling periods were quite similar. These conditions establish a riverine velocity of approximately $50 \mathrm{~cm} \cdot \mathrm{s}^{-1}$, an estuarine velocity of approximately $100 \mathrm{~cm} \cdot \mathrm{s}^{-1}$, and a tidal velocity on the order of $200 \mathrm{~cm} \cdot \mathrm{s}^{-1}$.

\subsection{Structure of the Thesis}

The goal of this thesis is to enhance the current understanding of shear stratified turbulence and two-layer hydraulic control theory through observations from an energetic and highly stratified estuary. The majority of the study focuses on the stationary front present at the mouth of the Fraser River during the late ebb. In addition, estuarine dynamics are addressed with respect to the temporal and spatial variability of mixing processes by quantifying buoyancy flux in the estuarine channel through a complete tidal cycle.

The thesis is organized as follows. An examination of the stationary frontal structure during the late ebb, including a survey of the local kinematics and an assessment of the relevance of two layer hydraulic theory, is presented in Chapter 2. Estimates of the entrainment velocity, and the vertical turbulent flux of both salt and momentum in the 


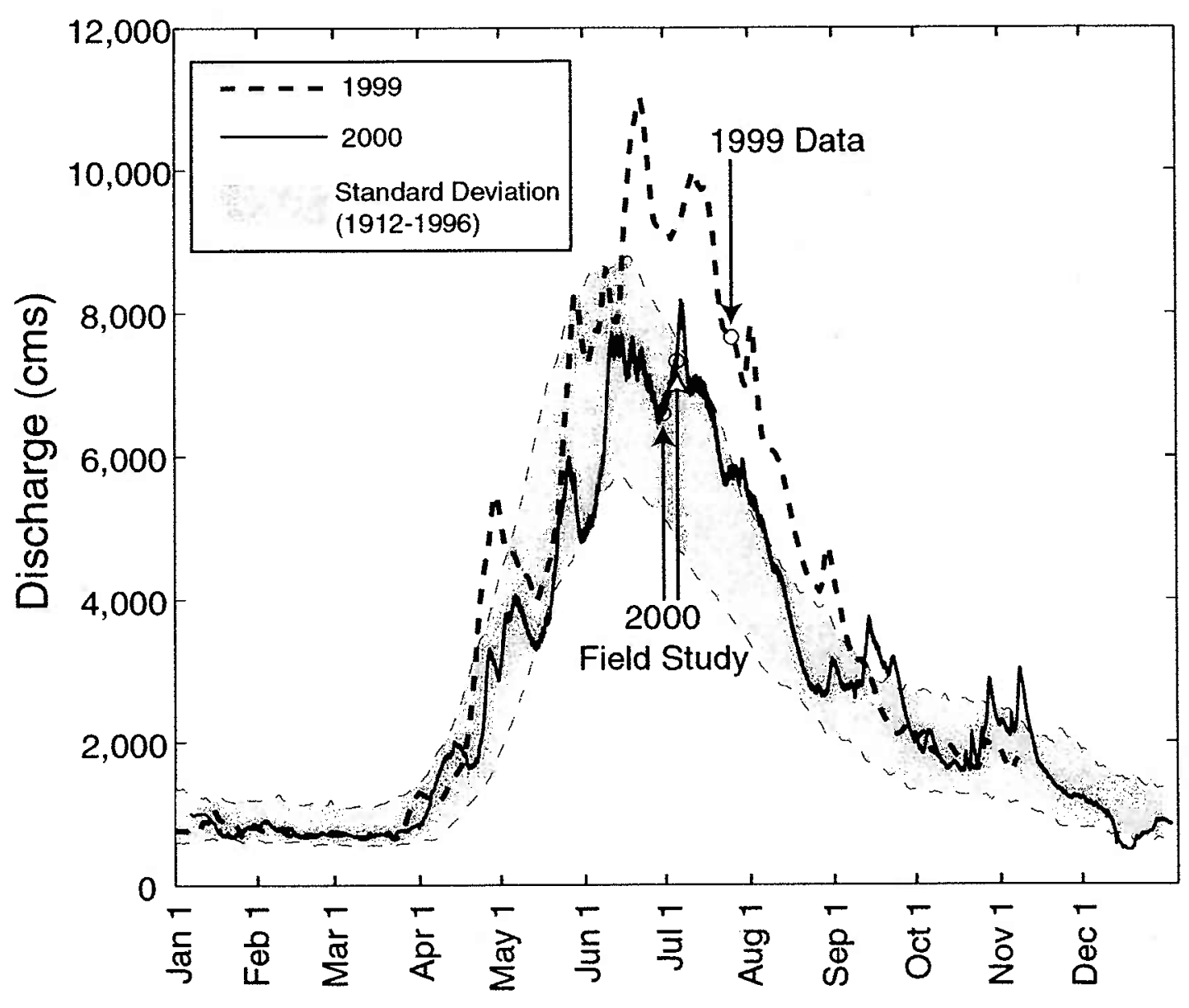

Figure 1.3: Fraser River discharge in cubic meters per second $\left(\mathrm{m}^{3} \mathrm{~s}^{-1}\right)$, as measured at Hope, approximately $150 \mathrm{~km}$ upstream of Sand Heads. Discharge curves are shown for 1999 and 2000, along with the standard deviation around the mean for historical flows from 1912-1996. Data collection periods are identified on the respective curves. 
region seaward of the stationary front are presented and discussed in Chapter 3. Chapter 4 focuses on the estuarine channel, evaluating the spatial and temporal variability of the mixing processes by quantifying buoyancy flux rates, and addressing the mechanism responsible for the retreat of the salt wedge during the ebb. A summary, and conclusions of the entire work, are presented in Chapter 5. Chapters 2 through 4 contain focused introductions that enhance the material presented here, and add detail specific to the chapter. 


\section{Chapter 2}

The Three-Dimensional Structure and Hydraulic Control of a Highly Stratified Estuarine Front 


\section{ABSTRACT}

Observations at the mouth of the Fraser River (British Columbia, Canada) indicate an abrupt frontal zone between unstratified river outflow and a highly stratified river plume, with differences in salinity greater than 25 psu across a few meters in the vertical and several hundred meters in the horizontal. The front roughly follows a natural break in the bathymetry, crossing the channel at an angle of approximately $45^{\circ}$, and is essentially stationary for a period of approximately 3.5 hours centered on the low tide following the larger of two daily ebbs. This chapter is focused on understanding the structure of the front and associated circulation during this period. The location of the front is coincident with observations of significantly supercritical internal Froude numbers at the front, based on velocities in the along-flow direction. However, because the front is oriented obliquely to the outflow, a coordinate system can be selected that is normal to the front, and for which a critical Froude number of one is obtained. This indicates that a Froude angle, similar in application to a Mach angle for trans-sonic flows, can be used to determine critical conditions when the front is oblique to the principal flow direction. A three-dimensional salt balance indicates that the salt feeding turbulent mixing processes seaward of the front is delivered to the region through a subpycnocline current flowing from the north in an along-isobath direction. This current exists independent of the fresh water discharge, in association with the ebbing tide within the Strait of Georgia. 


\subsection{The Near-Field Plume}

The shape of a river plume in the immediate vicinity of the river mouth can take different forms (Kashiwamura and Yoshida, 1967), depending on the interaction of inertial and buoyant forces within the flow field. The near field of the river plume can be defined as that portion of the plume where inertia dominates over buoyancy (Jirka et al., 1981), and Froude numbers are supercritical. The near field is typically a very small region in comparison to the far field, or buoyancy dominated, portion of the plume, yet it is a region of intense and dramatic change in the structure of the plume. Mixing processes and spreading characteristics initiated in the near-field are likely to have significant impacts in the far-field, by establishing the thickness of the plume and its initial dilution. The ramifications of the near-field dynamics also provide a physical foundation for other important biological and chemical processes (Yin et al., 1994).

A distinct frontal zone is a definitive characteristic of a highly stratified estuary, but less significant in well-mixed or partially mixed estuaries, as discussed in Chapter 1. In this regard, it is the presence of a strong river outflow that is typically responsible for establishing and maintaining a well-defined front (e.g., Geyer, 1988). An idealized crosssection through a highly stratified frontal zone is shown in Figure 2.1. Here, the nearfield and far-field regions are delineated, and the frontal zone is identified. The frontal zone is the point where the interface intersects the bottom. In Figure 2.1, a sharp interface between the two layers is shown; field observations typically identify an interface of some finite width across which the transition between water masses occurs. 


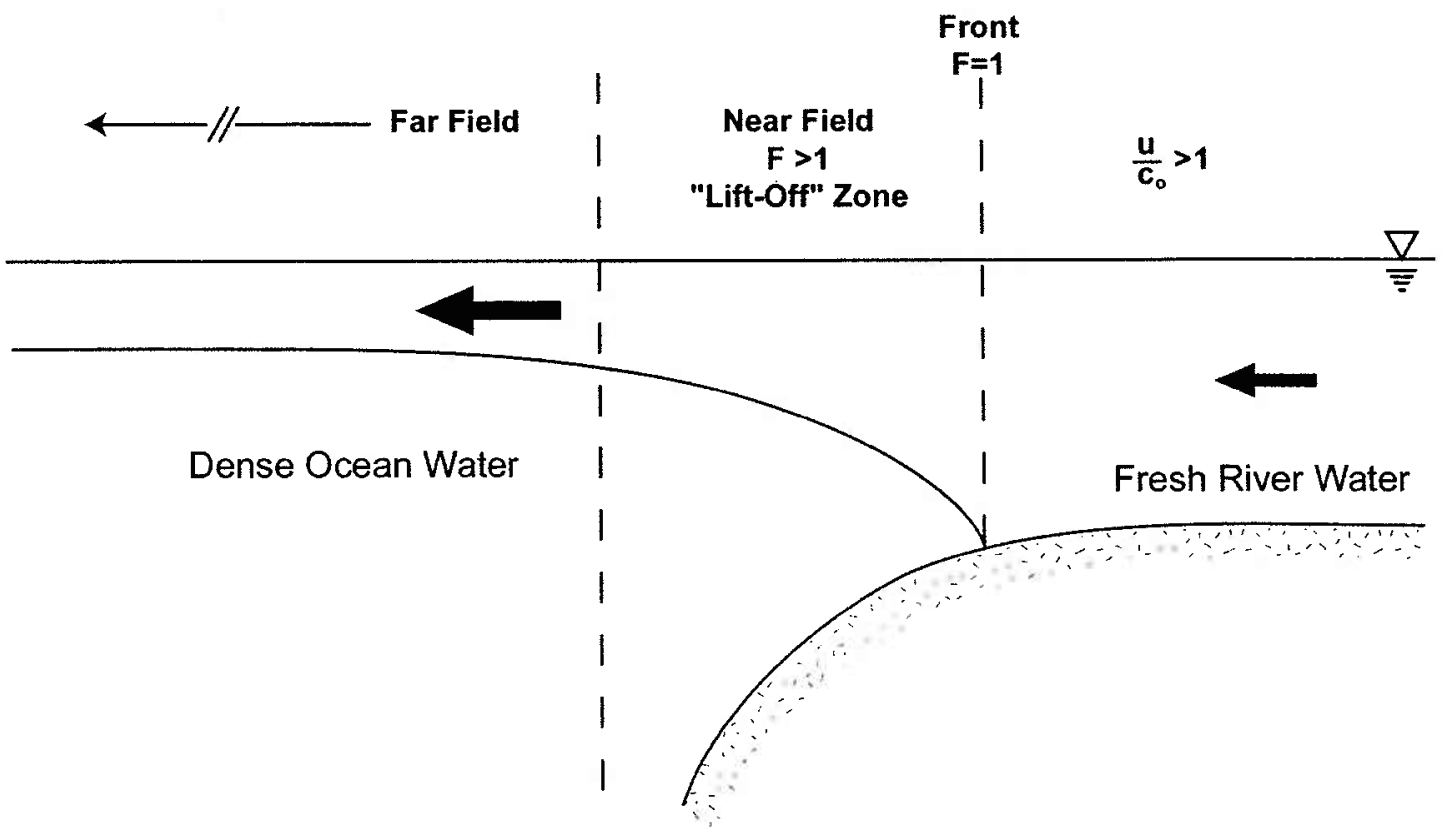

Figure 2.1: Definition sketch for front and near-field regions. Fresh river water is discharging to the ocean, establishing a front at the point where the internal Froude number, $\mathbf{F}$, of the upper layer flow is equal to one. The region immediately seaward of the front, where the depth of the interface is adjusting rapidly and Froude numbers are supercritical is considered the near field region and sometimes referred to as the "lift-off" zone. The far-field lies seaward of this adjustment region, and extends far downgradient. The near field physics are dominated by inertial influences. Buoyancy differences and external forcing (e.g., wind stress) dominate in the far field. In the region landward of the front, the ratio of velocity to a wave speed representative of the density difference at the front is greater than one. This ratio represents a "virtual" Froude number, illustrating that the location of the front is pushed sufficiently downslope to satisfy the critical condition. 
This chapter focuses on frontal zone structure and hydraulic control processes as well as near field plume dynamics, illustrated with data from the Fraser River Estuary. The front and near field of the plume comprise a region that will be further referred to as the "liftoff' zone because across this zone the river discharge loses contact with the bottom, and the interface rises rapidly in the seaward direction.

\subsubsection{Hydraulic Control}

According to two-dimensional, inviscid theory, the internal Froude number, which represents the ratio of flow velocity to internal wave speed, should be critical and equal to one at the front (Armi and Farmer, 1986; Farmer and Armi, 1986), thus providing a dynamic constraint on the front location, as shown in Figure 2.1. The composite Froude number, $G$, and layer Froude numbers, $F_{i}$, were defined in Chapter 1, as equation (1.2).

According to Armi and Farmer (1986) and Farmer and Armi (1986), the flow seaward of the front is supercritical (i.e., Froude number values greater than unity) as the upper layer thins and accelerates. Landward of the front, the velocity exceeds the phase speed $c_{o}$, as described in Figure 2.1. Thus the front is constrained by the deceleration of flow over the crest of the bathymetry, and is positioned at the precise point where the mean flow velocity is equal to $\sqrt{g^{\prime} H}$, where $H$ represents the water column depth.

Stommel and Farmer (1952) first suggested that a critical Froude number value of unity is necessary at the front of an arrested salt wedge, and provided a steady, inviscid two-layer analytical model for its development. Bowden (1967) reiterated the importance of the Froude number, suggesting that the flow in natural estuaries adjusts so that a critical Froude number of 1 is reached at the mouth. The rigorous analytical approach 
conducted by Armi and Farmer (1986), and Farmer and Armi (1986), provided similar results.

Given these conditions, a general deceleration of the flow would result in a landward, or upslope, migration of the front. This is seen at the beginning of the flood, as the front begins to migrate landward into the channel. In a similar manner, upper layer acceleration would result in a repositioning of the front further down slope.

Armi and Farmer (1986) draw a clear distinction between an arrested density front, and an advancing front as described by Benjamin (1968). Jirka and Masamitsu (1987) identify bottom friction as the key mechanism distinguishing the blunt shape of an advancing density current from the wedge-like shape of an arrested front. The lift-off front in the Fraser is essentially stationary for several hours, and is more characteristic of an arrested front. However, as the tide begins to flood and the salt wedge begins to migrate landward, the structure of the front transitions towards the blunt shape described by Benjamin (1968) and Jirka and Masamitsu (1987). The blunt frontal structure of the Fraser River salt wedge was observed as the front passed an upstream anchor station location. The anchor station data is discussed in more detail in Chapter 4.

\subsubsection{Structure of River Plumes}

Seaward of the front, within the near-field region of the plume, the upper layer thins and expands laterally. Various researchers have studied the dynamics of this region over the past 50 years, and several models have been used to elucidate the near-field plume expansion and structure. Bates (1953) treated a river plume as a turbulent jet spreading laterally above a passive salt-water mass, and suggested that lateral mixing was 
principally responsible for entrainment. Takano $(1954,1955)$ introduced another early model, which treated the river water as a homogeneous layer expanding laterally between hyperbolic boundaries and thinning in the vertical. Both of these models ultimately provided unsatisfactory descriptions of the near-field expansion due to their neglect of buoyancy. Although inertia generally dominates over buoyancy in this region, the effect of buoyancy cannot be neglected. This is reflected by the importance of the Froude number to the dynamics of the region, and the observation that the Froude numbers, although generally supercritical, are still of order one.

Bondar (1970) provided a description of plume expansion that combined buoyancy effects, expressed through a surface elevation anomaly, with streamwise inertia. Wright and Coleman (1971) extended this idea by showing the importance of vertical mixing to the streamwise momentum budget, and thus the observed spreading rate.

More recent research has focused on the structure of the near-field plume, particularly with regards to the relationship between the expansion rate and the threedimensional structure of the frontal zone. Atkinson (1993) used numerical simulations to focus on the case of a surface discharge over a shallow sloping bottom. The results of these simulations, and similar laboratory experiments by Adams and Stolzenbach (1977), have indicated that the interplay between buoyant and inertial forcing can result in a wedge shaped detachment, with the bottom attached region tapering in width in the seaward direction. This geometry is manifested by the tendency for the upper portion of the discharged fluid to spread laterally, and the lower portion to rise. This allows denser ambient fluid to move in from the sides, generating a frontal structure at an oblique angle 
to the streamlines of the discharging fluid. The Adams and Stolzenbach (1977) model of detachment indicates that lateral mixing may be important for certain flow geometries. This stands in contrast to Wright and Coleman (1971), where vertical mixing was observed to be responsible for the majority of entrainment in the Mississippi plume. The aspect ratio, or ratio of depth to width, of the Mississippi plume is several orders of magnitude smaller than the laboratory flows investigated by Adams and Stolzenbach (1977), which may be more directly relevant to smaller scale industrial discharge plumes, or to localized regions of river plumes due to the local geometry. In general, the relative importance of vertical mixing processes should decrease with width so that at larger aspect ratios, lateral mixing processes should be more important.

The Fraser River provides an ideal field setting in which to investigate the dynamics discussed above. This chapter is focused on the near field lift-off dynamics of the highly stratified front that sets up at the mouth of the Fraser River during the end of the ebb portion of the tidal cycle. A main goal of the chapter is to assess the relevance of twodimensional hydraulic theory (e.g., Farmer and Armi, 1986) to three-dimensional frontal structures, such as the front observed at the mouth of the Fraser. A second goal of the chapter is to delineate the kinematics associated with the local salt balance at the front, and to identify the source of the entrained salt. Here, the relative importance of lateral and vertical mixing processes in the Fraser lift-off will be discussed. Finally, the observed expansion and near field plume structure at the mouth of the Fraser is considered with respect to the existing models and observations discussed above. 


\subsection{The Fraser Estuary and the 2000 Field Effort}

The mouth of the Fraser River consists of a channel that is confined by a jetty along its northern boundary, with shallow tidal flats to the south, and an average channel depth on the order of 10 to 15 meters, as shown in Figure 2.2. The jetty along the northern portion of the channel ends at Sand Heads, allowing lateral expansion of the outflow to the north. On the south side, tidal flats continue $1.5 \mathrm{~km}$ seaward beyond Sand Heads. Approximately 0.5 to $1 \mathrm{~km}$ beyond Sand Heads the bathymetry breaks sharply, reaching depths greater than 50 meters in less than 500 meters in the along channel direction. This bathymetric break is situated at an angle of roughly $45^{\circ}$ to the channel, and the foreslope is characterized by an irregular pattern of gullies with scales on the order of 100's of meters.

The dynamics in the estuary are driven by an influx of buoyancy and inertia from the river discharge, and the tides in the Strait of Georgia. The highly energetic estuarine environment set up by the interaction of these three strong forcing mechanisms creates intense stratification and an energetic turbulent environment. The resulting salt wedge is characterized by salinity differences of greater than 20 psu across only a few meters in the vertical and less than 500 meters horizontally at its head. The salt wedge advances landward some 15 to $20 \mathrm{~km}$ into the channel on each tidal cycle, only to be flushed back to the mouth daily during the strong ebb (Geyer and Farmer, 1989). At this point in the cycle, after the salt wedge has retreated to the mouth, there is a period of quasi steadystate dynamics as the front location remains stable for several hours, prior to beginning 


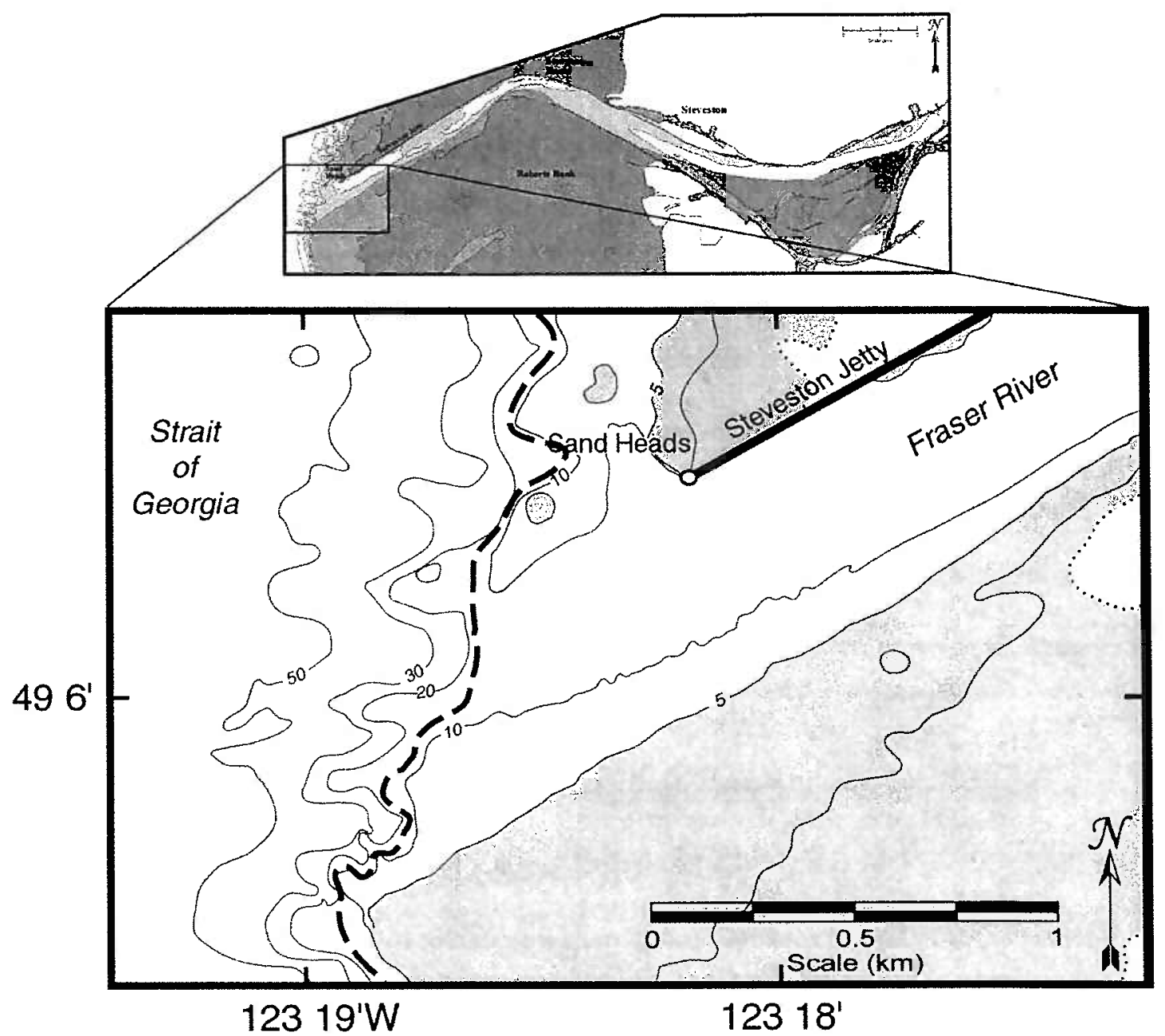

Figure 2.2: Estuarine channel, and mouth regions of the Fraser River Estuary. In the bottom panel, the bold dashed line represents the location of the $12 \mathrm{~m}$ bathymetric contour, which serves as a marker for the bathymetric break. Contours are shown in meters, and are based on a compilation of bathymetric data from Canadian Hydrographic Service (Chart 3490, 1997), and shipboard measurements (July 2002). 
its next advance into the channel on the flooding tide. This chapter focuses on the structure and dynamics of the lift-off zone during this period.

The 2000 study was timed to coincide both with the freshet and spring tides, and was conducted between June 30 and July 4, 2000. Data was collected from shipboard instrumentation, including two hull-mounted ADCPs, operating at $1200 \mathrm{kHz}$ and 300 $\mathrm{kHz}$, and a towed Ocean Sensors 200 Series CTD unit. Three to five parallel passes, oriented in the along-channel direction, and spaced evenly across the channel, were used to identify the three dimensional structure of the lift-off zone. Strong velocities in the along-channel direction precluded the towing of instruments on cross-channel transects. The seaward extent of the data transects rarely exceeded $2 \mathrm{~km}$ beyond Sand Heads, as the focus of the study was confined to the front and the near field of the plume.

\subsection{Structure of the Lift-Off Zone}

There are two topographic features that should affect the position of the front: the sharp bathymetry break, and the end of the jetty along the north side of the channel at Sand Heads. The presence of the sharp break in bathymetry should provide a fixed seaward limit for the location of the front, due to the large mass of salt water positioned below the break, through mechanisms similar to the hydraulic control influences of a sill described by Farmer and Armi (1986). The end of the jetty represents a relaxation of the lateral constriction confining the river discharge to the channel and allows the less dense fresh water to spread laterally to the north. This expansion of the channel should also influence hydraulic control, as discussed by Armi and Farmer (1986). The presence of a 
source of ambient ocean water at the edge of the discharge plume, seaward of Sand Heads, may hasten the lift-off through lateral impingement mechanisms (e.g., Adams and Stolzenbach 1977).

The structure and dynamics of the lift-off zone are established by these local bathymetric features. Later sections of this chapter investigate these influences in more detail, through determination of local Froude numbers and an analysis of hydraulic control. First, observations of the lift-off zone structure are described.

\subsubsection{Streamlines of the Upper Laver}

Data from five passes during the late ebb on June 30 were used to construct upper layer streamlines for the lift-off region, as shown in Figure 2.3. Velocity data from the five passes were interpolated laterally using an elliptic interpolation scheme that preferentially weighted values located along a major axis, which in this case was aligned roughly with the bathymetry break. In this scheme, distances perpendicular to the defined major axis are scaled by a constant value (with a factor of ten typical for the liftoff analyses), then all the available data is averaged using a weighting factor dependent on the scaled distance raised to a constant power (typically 0.1). After a series of twodimensional interpolations along horizontal planes at $1 \mathrm{~m}$ depth intervals, velocities were averaged vertically across the upper layer, which was defined as all fluid with salinity less than $20 \mathrm{psu}$, and streamlines were generated from the resulting velocity grid. The direction of the streamline was defined parallel to the mean velocity at each point, such that the mean cross-streamline velocity was equal to zero. 


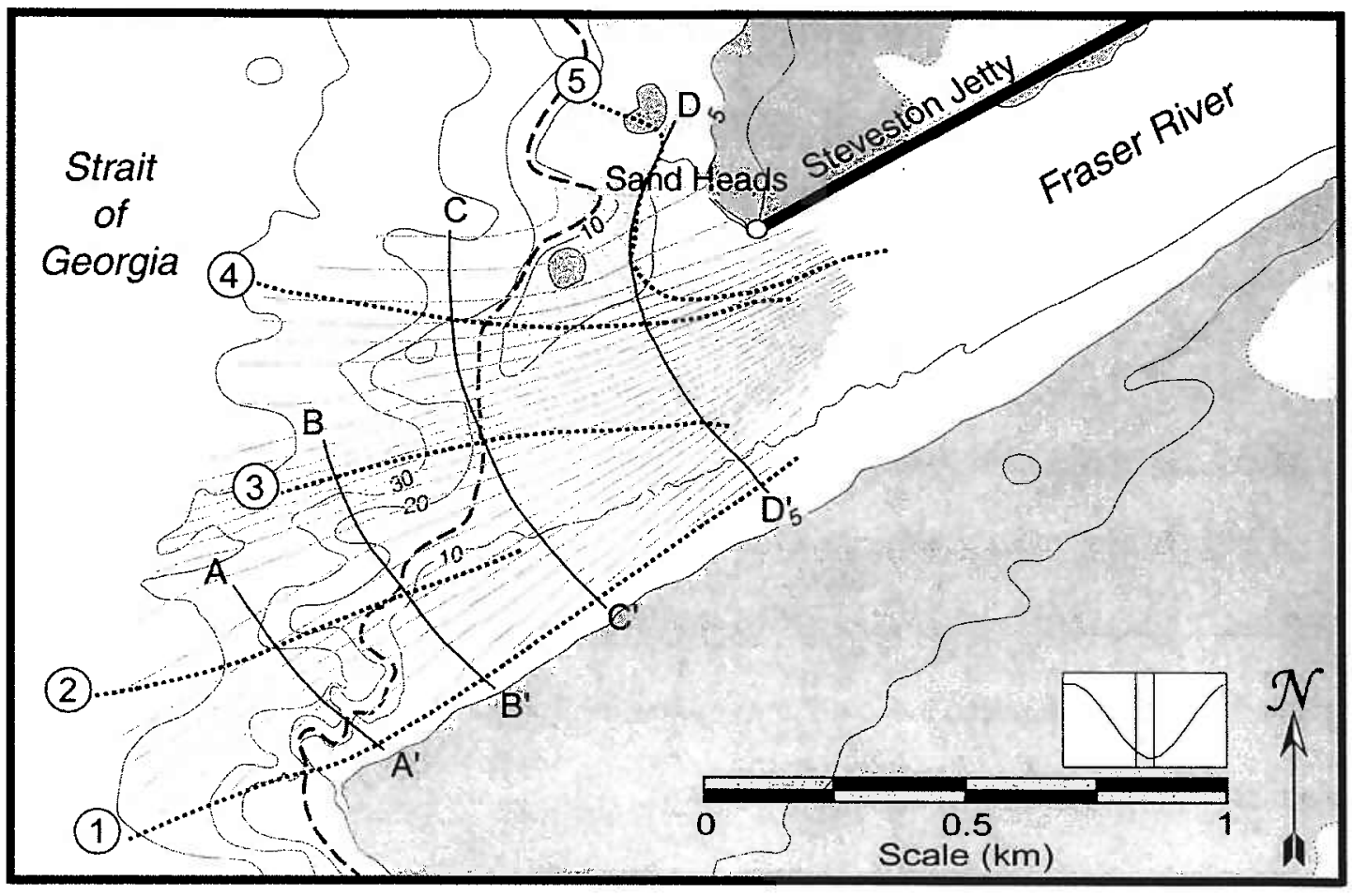

Figure 2.3: Mouth of the Fraser, showing upper layer streamlines and the location of cross sections shown in Figures 2.4 and 2.5. Streamlines are shown as the solid gray lines, and cruise tracks are identified as the heavier dotted lines. The heavy dashed line delineates the location of the $12 \mathrm{~m}$ isobath, and the approximate location of the bathymetric break. Cruise tracks numbered 1 through 4 are shown in cross section in Figure 2.4. Transverse cross sections A-A' through D-D' are shown in Figure 2.5. Streamlines are generated from $\mathrm{ADCP}$ velocity data collected along the identified cruise tracks. Data was collected during the late ebb, as shown in the inset frame. Streamline normals are shown behind the streamlines, and together with the streamlines create a natural curvilinear coordinate system. 
A new coordinate system was generated from the established streamlines by defining grid lines normal to the streamlines, and measuring distances along the deformed streamlinenormal grid mesh. The normals are shown on Figure 2.3 as dashed lines. This new coordinate system facilitated calculations in the lift-off region due to alignment with the upper layer flow direction.

\section{$\underline{\text { 2.3.2 Lift-Off Cross Sections }}$}

The three dimensional structure of the lift-off zone is illustrated with a series of cross sections. Eight cross sections through the lift-off zone are indicated on Figure 2.3, and shown in profile in Figures 2.4 and 2.5 representing the along-channel, and acrosschannel structure, respectively.

The series of along-channel sections shown in Figure 2.4 indicate that stratification is high, and relatively uniform across the channel. This is evidenced by the similarly close spacing of the isohalines in each panel. The front itself is defined by the bottom intersection of the isohalines. It is relatively sharp across the channel, although somewhat less so in the center of the channel, where velocity-shear driven mixing processes may broaden the gradient zone.

The cross-channel sections of Figure 2.5 demonstrate the inclination of the isohalines, which slope upwards to the north. Discharge is dominated by a high velocity region in the center of the plume that spreads laterally and shoals in the seaward direction. The cross-channel velocity structure generally exhibits characteristics of an exchange flow, with surface flows directed northward, and deeper flows moving towards the south. The deeper flows are associated with the ebbing tide in the Strait of Georgia, 

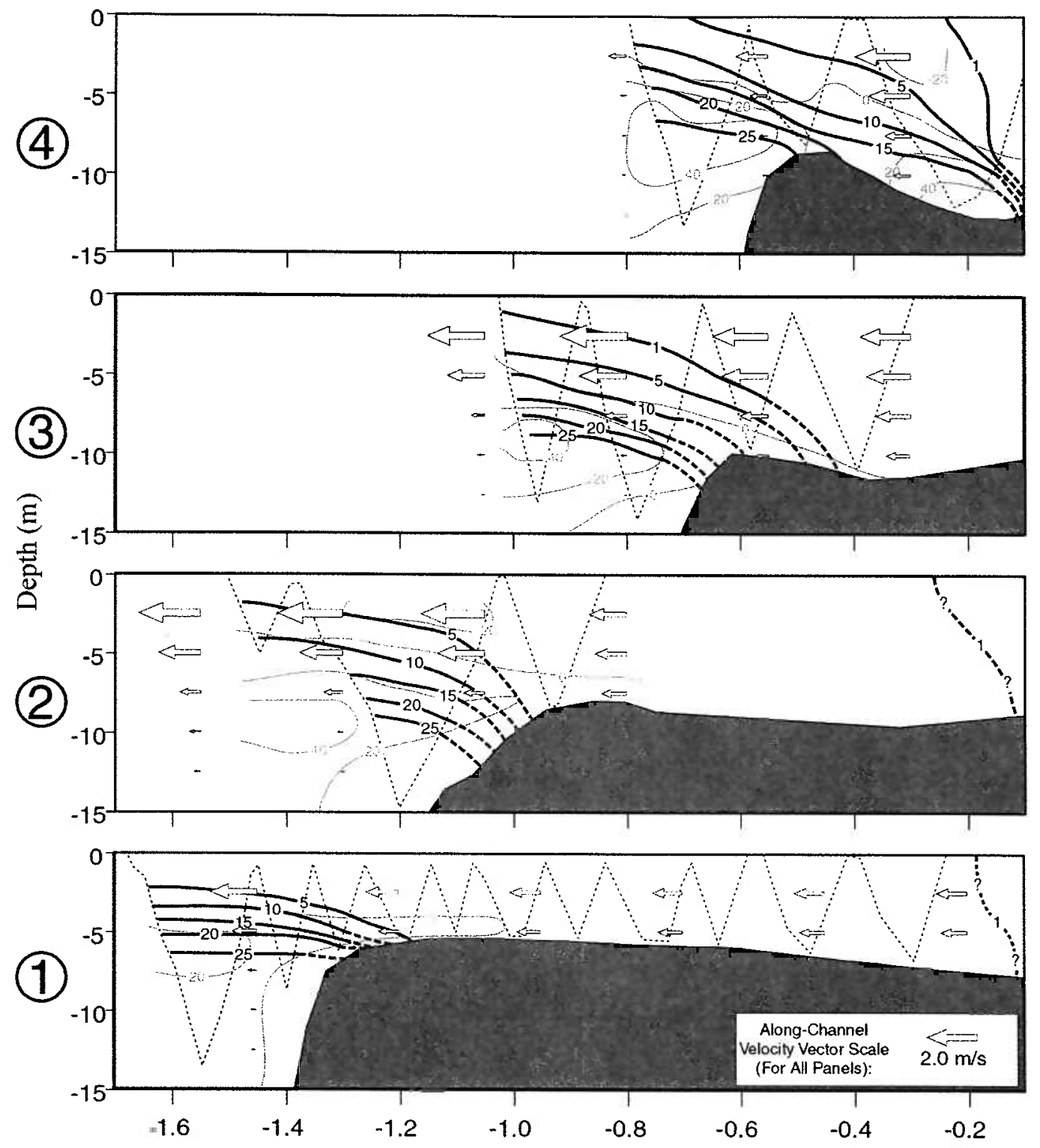

Along Channel Coordinate ( $\mathrm{km}$, origin at Sand Heads, positive landward)

Figure 2.4: Along channel cross-sections through the lift-off zone along cruise tracks as shown in Figure 2.3. Salinity contours $(1,5,10,15,20$, and $25 \mathrm{psu})$ are shown in black, with arrows representing the magnitude and direction of the streamwise velocity component. Cross channel velocity (i.e., along the streamline normals shown dotted in Figure 2.3) is represented by the gray contours, in units of $\mathrm{cm} \cdot \mathrm{s}^{-1}$, with positive values representing southward directed flows. The dotted line represents the approximate path of the towed CTD unit. 


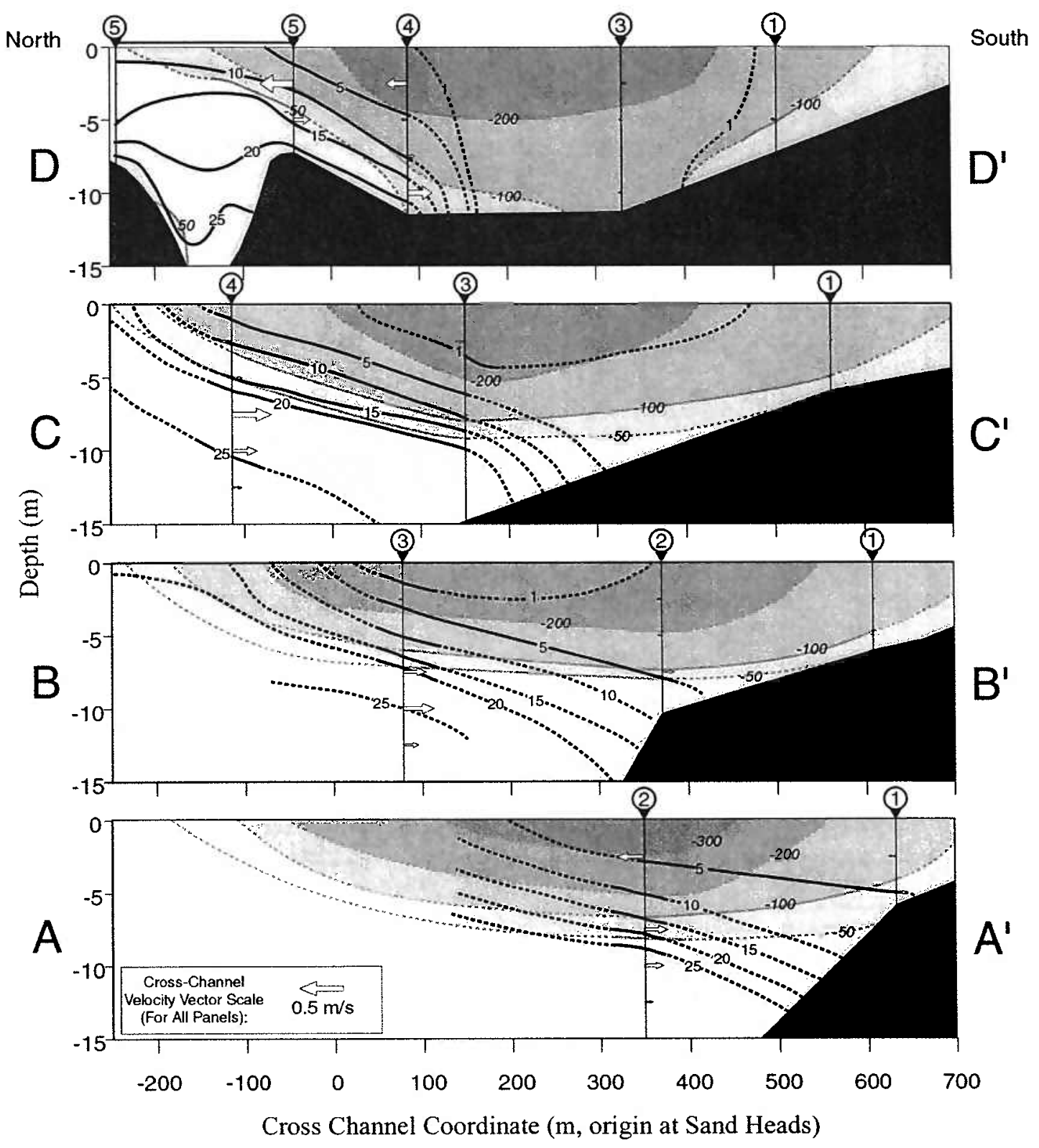

Figure 2.5: Transverse channel cross-sections through the lift-off zone at progressively seaward positions as identified by the cross-section locations in Figure 2.3. The ordinate axis represents crosschannel distance, in meters, from an origin aligned with Sand Heads. Positive values are directed in a generally southward direction (normal to the Steveston Jetty). Salinity contours $(1,5,10,15,20$, and $25 \mathrm{psu}$ ) are shown in black, with arrows representing the magnitude and direction of the cross-channel velocity component (i.e., along the streamline normals shown dotted in Figure 2.3). Streamwise velocity is represented by shading and contours, in units of $\mathrm{cm} \mathrm{s}^{-1}$. The black triangles and numbers at the top of each cross section indicate the location and number of the cruise tracks from which data for these cross sections is extracted. The region between the two connected triangles in cross-section D-D' lies along a cruise track, as shown in Figure 2.3. Dashed lines represent visual extrapolations of the observed data. 
when currents are directed south and west through the Strait of Juan de Fuca to the open Pacific Ocean.

\subsubsection{Front Location and Temporal Variability}

As indicated in Figure 2.4, the bottom front is relatively compact in the along channel direction. This is illustrated again on Figure 2.6, where the frontal zones for the early and late portions of the lift-off period are delineated by the width of the region where water with salinities of 5 psu to 20 psu intersects the bottom. The shaded region in Figure 2.6 is based on observations from several different days, and may overestimate the width of the frontal zone due to the horizontal resolution of the CTD tow-yo passes, as indicated by the dotted lines in Figure 2.4 .

In addition to the compact nature of the frontal zone, one of the most striking characteristics is its oblique orientation with respect to the channel and the along stream direction. Across the southern half of the channel, the frontal zone appears to be relatively uniform in width and orientation. A bulging area is observed in the northcentral portion of the channel, where there is a broad shoaling region landward of the bathymetric break. At the northern end of the frontal zone, the bottom attachment of isohalines is tightly grouped, similar in width to the southern portion of the front, but the front is pushed landward of the bathymetric break and Sand Heads. In this region, the intrusion of high salinity fluid in the vicinity of Sand Heads is made possible by the existence of a scoured trench just seaward of the end of the jetty.

Comparison of the two panels in Figure 2.6 indicates that the front tends to become more uniform in structure during the beginning of the flood. Although the bulging region 

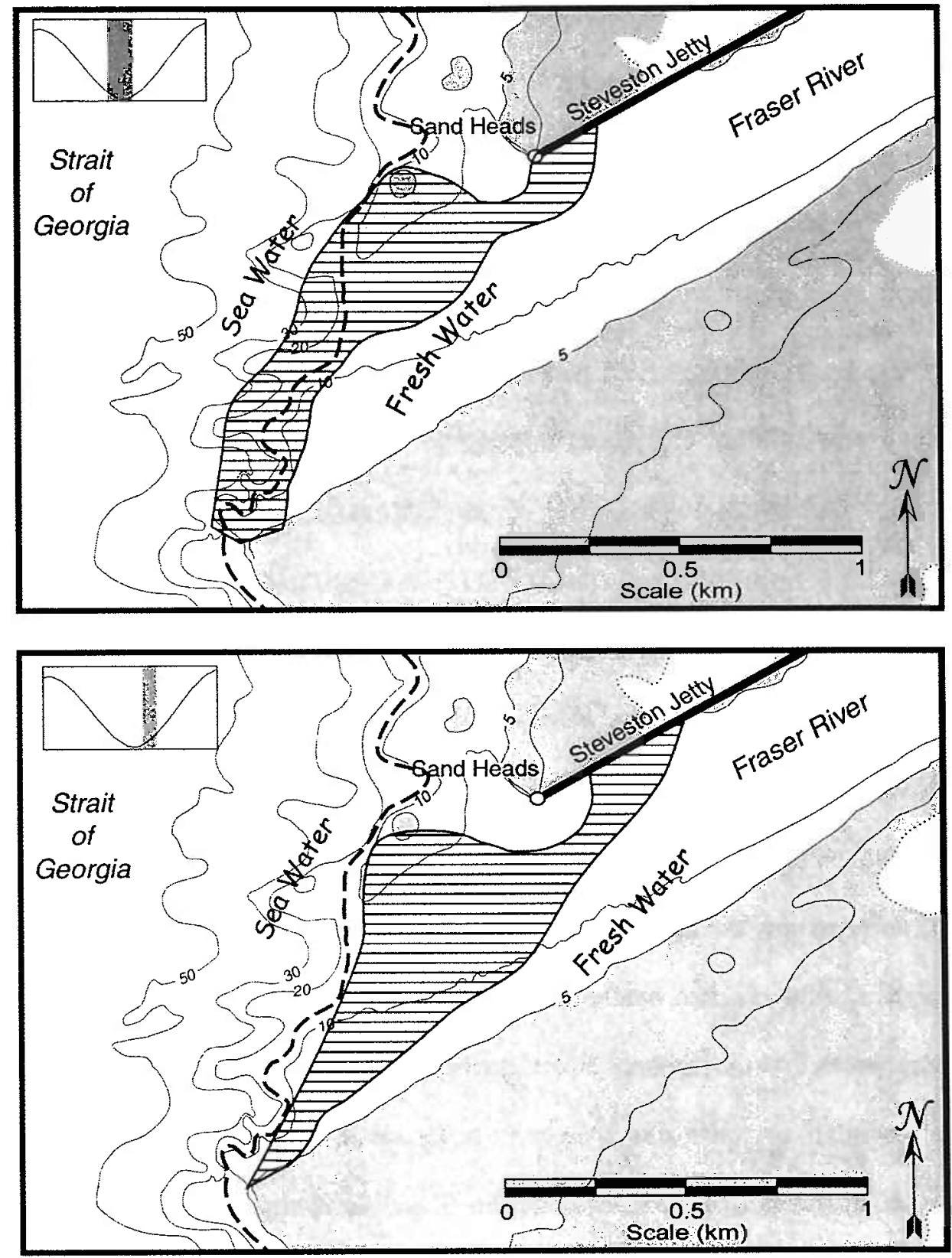

Figure 2.6: Width of frontal zone as defined by presence of bottom water between 5 and 20 psu during the early and late stages of the lift-off period as shown in the tidal curve inset in each upper left hand corner. The hatched regions are based on data observed during multiple passes through the lift-off zone between June 30 and July 3, 2000. For each pass, the landward edge of the region was represented by the most seaward bottom water sample with salinities less than 5 psu. The seaward edge is defined by the most landward observation of water with a salinity greater than $20 \mathrm{psu}$ at any point in the water column. The limits of the frontal zone are drawn liberally to encompass the observed front locations, and without regard to local bathymetry. Data for the 20 psu boundary are shown on Figure 2.7. 
in the north central portion of the front is still evident during the later period, the leading edge of the frontal zone appears to be established by an influx of salt from both the central portion of the channel and the scoured trench around Sand Heads.

There appears to be a significant amount of variability in the position of the frontal zone at scales on the order of $100 \mathrm{~m}$. This is best illustrated by considering the observed bottom attachment of a single isohaline. In Figure 2.7, the bottom attachment of the 20 psu isohaline is shown for both early and late stages of the lift-off period, as in Figure 2.6. Here, the raw observations are represented by the closed and open circles, which represent inherent uncertainty in the data based on the horizontal resolution of the CTD tow-yo passes. The hatched region is drawn to encompass this data without regard to bathymetry. The resulting region, which is relatively broad, can be further constrained by assuming that variations in local bathymetry play an important role in the structure of the front. The bold line provides a location for the 20 psu isohaline bottom attachment that is consistent with the observations and follows local isobaths where possible. Note that the channels and gullies that are present along the bathymetric break are well correlated with the observed front locations, suggesting that these features contribute substantially to the spatial uncertainty suggested by the rigid interpretation of the data shown by the hatched regions.

A potential second source of this spatial uncertainty could be related to temporal variations in the frontal location, as the accumulated data span across several hours of the tidal cycle and are compiled from observations from several different days. The temporal evolution of the front was evaluated by comparing all front locations observed during the 

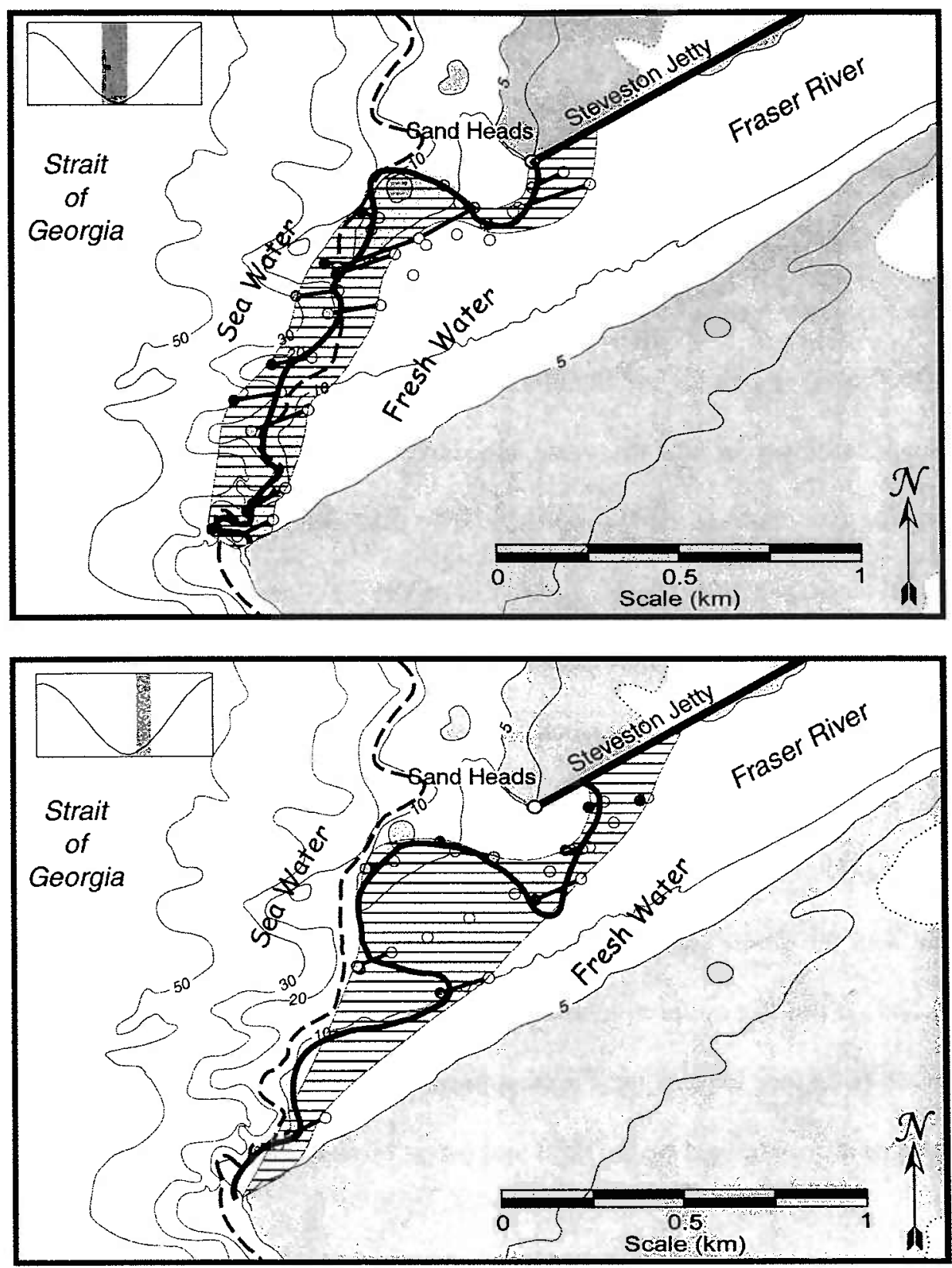

Figure 2.7: Maps of the intersection of the 20 psu isohaline with the bottom based on the same data set used to draw the frontal zones in Figure 2.6. The two panels represent the early and late stages of the lift-off period as shown in the tidal curve inset in each upper left hand corner. Open and filled circles represent definitive locations on the landward and seaward sides, respectively, of the 20 psu bottom attachment. The hatched area represents an envelope for bottom attachment of the 20 psu isohaline, based only on the observations, without regards to local bathymetry. The bold line represents an approximation of the front location based on both the observations, and the local bathymetry. 
five-day field study, within the three-hour period each day centered on the low tide following the largest daily ebb. Time dependent variability of the front location was found to be of the same order or smaller than, and indistinguishable from, the spatial variability discussed above. The correlation between the observed front locations and the bathymetry, as shown on Figure 2.7, however, suggests that most of the variability is driven by spatial irregularities in the bathymetry. In any case, a frontal velocity on the order of 50 meters per hour $(0.014 \mathrm{~m} / \mathrm{s})$ was observed during the lift-off period. This compares with front propagation speeds during the early flood on the order of 1,800 meters per hour $(0.5 \mathrm{~m} / \mathrm{s})$, as observed on several occasions.

\subsection{Control of the Front}

According to two-layer hydraulic theory, as discussed in Armi and Farmer (1986) and Farmer and Armi (1986), the front is established at the point where the fluid velocity and local internal wave speed are equal, resulting in an internal Froude number of one. However, the geometries considered in the analytical solutions of Armi and Farmer (1986) and Farmer and Armi (1986) are essentially two-dimensional, and may not be directly applicable to systems where variations in the cross-channel dimension are important, such as the Fraser lift-off region. This section investigates the relevance of the Armi and Farmer (1986) and Farmer and Armi (1986) solutions to the front observed at the mouth of the Fraser River, as shown on Figures 2.6 and 2.7.

Froude numbers were calculated for the lift-off region using a depth-averaged, upper layer velocity, which incorporated extrapolated values for the upper two meters of the 
water column not measured by the ADCP. The Froude numbers were based on velocities aligned in the direction of the upper layer streamlines, as they were calculated using the total magnitude of the depth-averaged velocity vector. The calculations assumed a layer interface coincident with the 14 psu isohaline surface. This choice represents the midpoint of the salinity range ( 0 to $28 \mathrm{psu}$ ), and is also supported by the velocity structure within the immediate area of the bathymetric break. The lower layer Froude number is nearly zero due to low velocities or velocities orthogonal to the streamwise flow across most of the region, so the composite Froude number is essentially equal to the upper layer Froude number. Landward of the front, "virtual" upper layer Froude numbers were calculated using a representative value of $g^{\prime}$, and the total water depth, as suggested in Figure 2.1. The results of these calculations are shown in Figure 2.8, where contours of the upper layer Froude number are plotted across the lift-off zone using data from June 30, 2000.

The contours shown in Figure 2.8 indicate that the flow is supercritical across the entire lift-off region, with the front stable at a Froude number in the vicinity of 1.5 across the majority of the channel. Supercritical velocities occur significantly landward of the front, near the end of the confined channel at Sand Heads. Even considering potential errors, this a significant departure from an expected Froude number equal to unity at the front. Prior to exploring the implications of these observations, a review of the calculations and alternative methods for interface determination is warranted. 


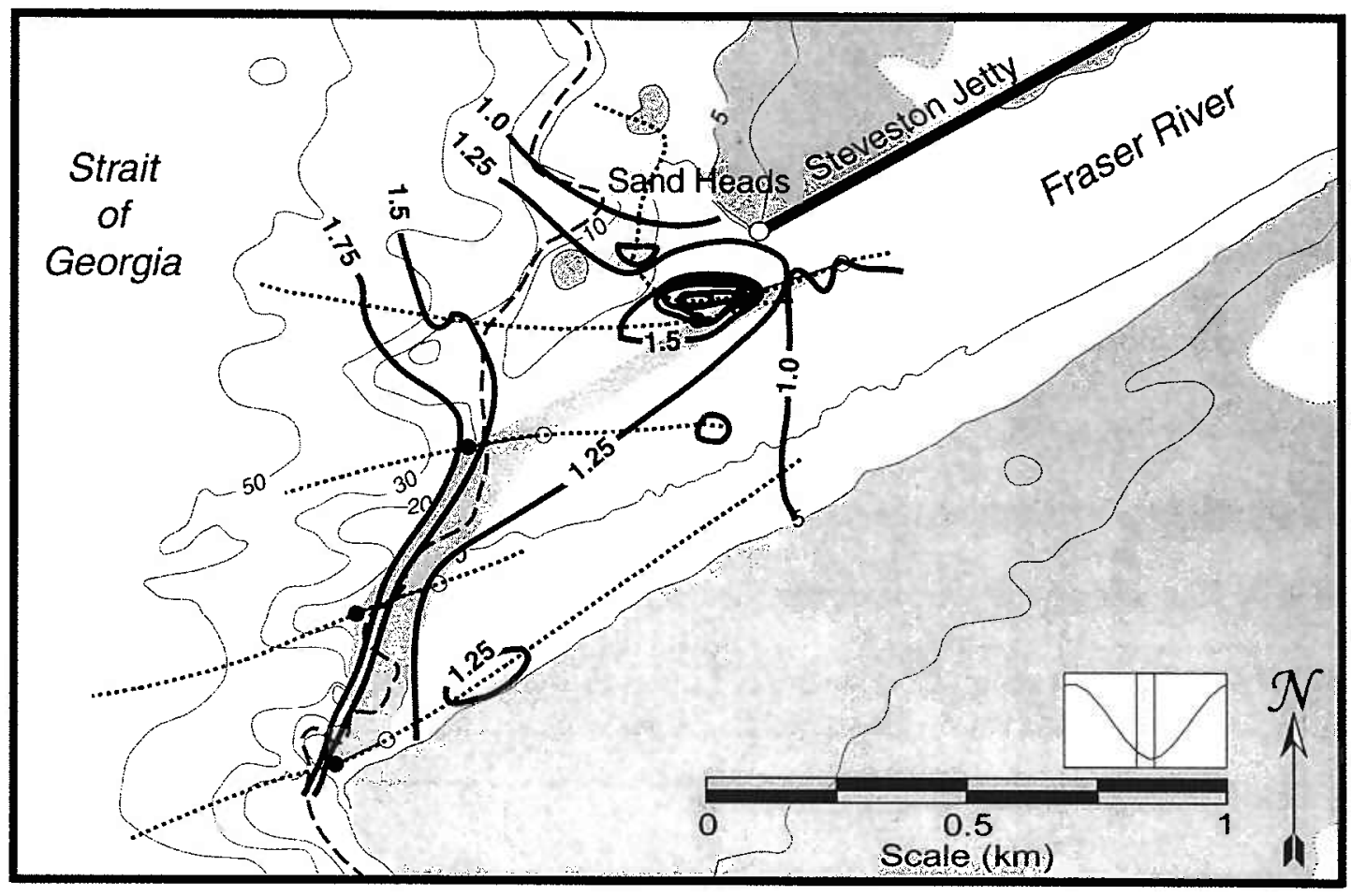

Figure 2.8: Upper layer Froude number across the lift-off zone during the late ebb on June 30, 2000. The 14 psu front is defined by the open and closed circles (as in Figure 2.7), and the shaded region. Upper layer Froude numbers across the region are estimated using a representative value of $g^{\prime}$. The layer interface was defined as the location of the 14 psu isohaline. Dotted lines represent ship tracks. Dashed line represents the $12 \mathrm{~m}$ isobath, as in Figure 2.3. 


\subsubsection{Interface Height Determination}

The Froude numbers characterized on Figure 2.8 were calculated from mean layer velocities averaged across a layer depth determined by the location of a particular isopycnal, in this case $14 \mathrm{psu}$. It is possible that this isopycnal does not adequately describe the dynamically important surface meant to approximate the sharp distinction between two distinct layers, and that a different isopycnal, or some other means of identifying the surface should be used. As an alternative to selecting an isopycnal, the velocity structure can be used to define the interface. A layer interface can be defined by identifying the point of the most severe transition in a vertical velocity profile, or the peak in the second derivative of velocity, $\frac{\partial^{2} u}{\partial z^{2}}$.

An interface defined in this manner is shown with vertical profiles of the streamwise velocity on Figure 2.9, superimposed over the transect 3 cross section from Figure 2.4. This estimate of the interface elevation demonstrates a bottom attachment very near the lip of the bathymetry break, as identified in the figure, and coincident with isohalines in the range from 10 to $15 \mathrm{psu}$. The lack of significant velocity gradient below this level indicates that the effective interface elevation must be at this point or higher. For the determination of the Froude number near the lift-off point, the choice of a layer interface coincident with the $14 \mathrm{psu}$ isohaline surface is consistent with the velocity structure. Further seaward, the velocity defined interface in Figure 2.9 quickly crosses isohalines until stabilizing between 20 and 25 psu no more than 150 meters behind the front. A hypothetical lift-off location, corresponding to the interface depth at which a critical 


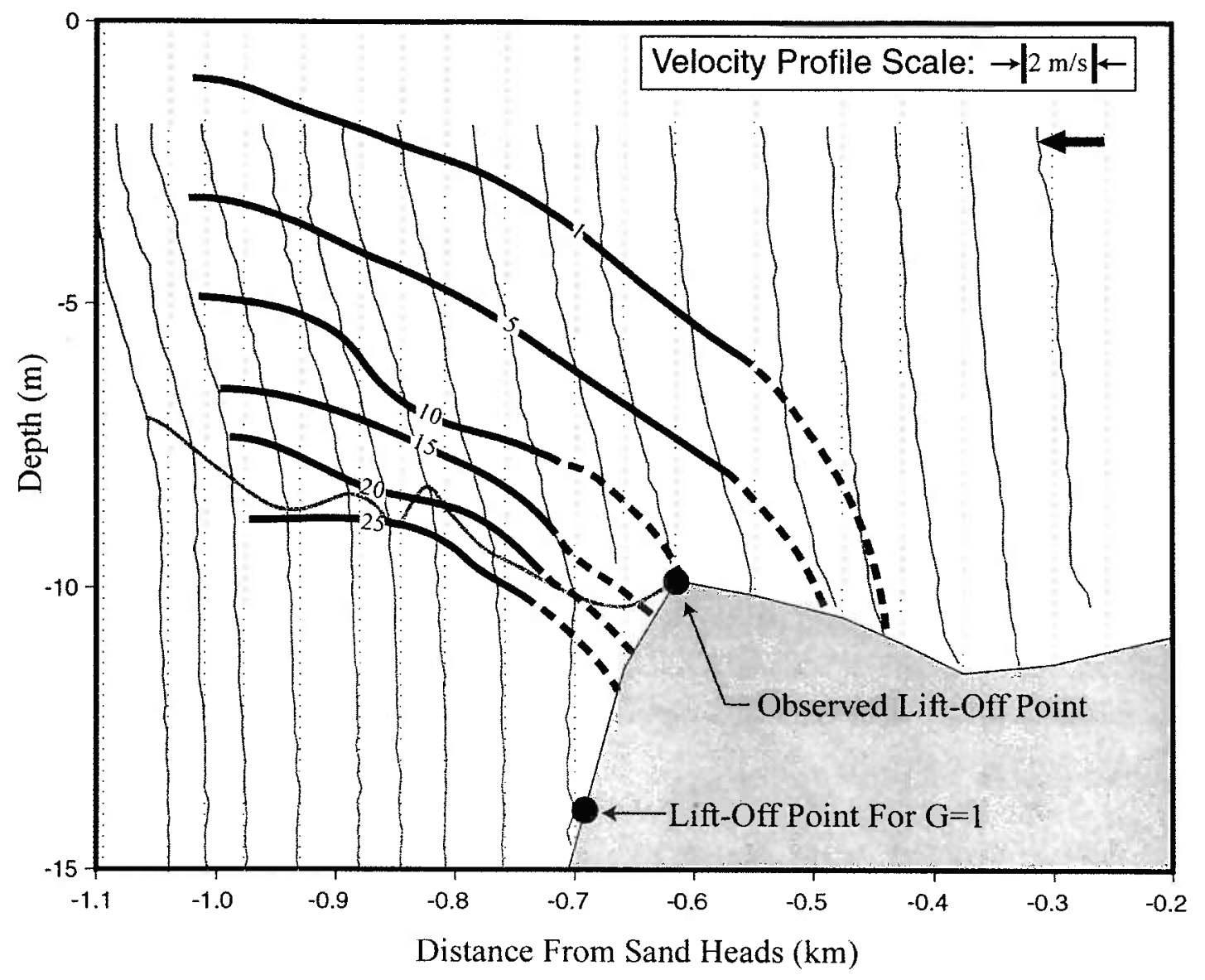

Figure 2.9: Streamwise velocity profiles overlaid on the lift-off cross section identified as transect (3) in Figure 2.3. The bold gray line represents the interface boundary as defined by a visual assessment of the point of the most abrupt change in the slope of the velocity profiles. At locations where robust velocity data does not extend deep enough, a lowest case estimate has been made by extrapolating the observable slope to $u=0$ (dotted lines). The two filled circles indicate the difference between the observed lift-off point, using the visual method just described, and the lift-off point required for a streamwise upper layer Froude number of 1 (using a vertical mean of the streamwise velocity). In order to meet the constraint of $G=1$, a significant deflection of the isohalines near the steeply soping portion of the bottom would be required. 
Froude number could be obtained using the observed velocity structure, was also identified and is shown on Figure 2.9. This downslope location is not consistent with the observed data.

\subsubsection{Froude Angle}

The concept of the Froude number is typically considered in one dimension, parallel to the orientation of the local streamlines. The two-dimensional structure of these transcritical flows can be understood by considering the growth of a wave front emanating from a point of disturbance in a moving flow (e.g. Liggett, 1994, pp. 319-323). Because the Froude number is dependent on depth, this two dimensional structure is sufficient for describing flows in three dimensions, such as the hydraulic control established at the mouth of the Fraser.

Under critical flow conditions, the flow velocity matches the wave speed, and the upstream edge of the wave front remains fixed at the point of the disturbance as shown in Figure 2.10 (a), while the radius grows with time. Under supercritical conditions, the entire disturbance is swept downstream, but the envelope of information transmittal is not limited to the axis of the flow direction, and defined by the difference between the ambient flow velocity and the wave speed, as in the one dimensional case. Here, as shown in Figure 2.10 (b), the envelope of influence of the disturbance can be identified by the half-angle formed between the direction of the ambient flow and a line emanating from the point of the disturbance tangent to the wave circle at all times. This angle, the Froude angle, is equivalent to the Mach angle from supersonic flow theory (Garvine, 


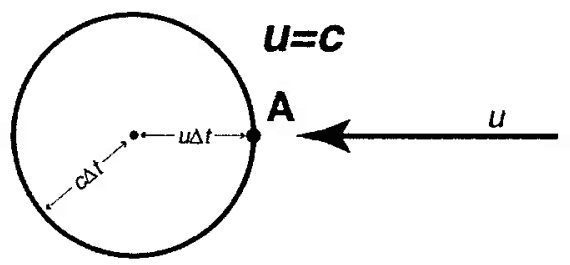

(a) Critical Flow
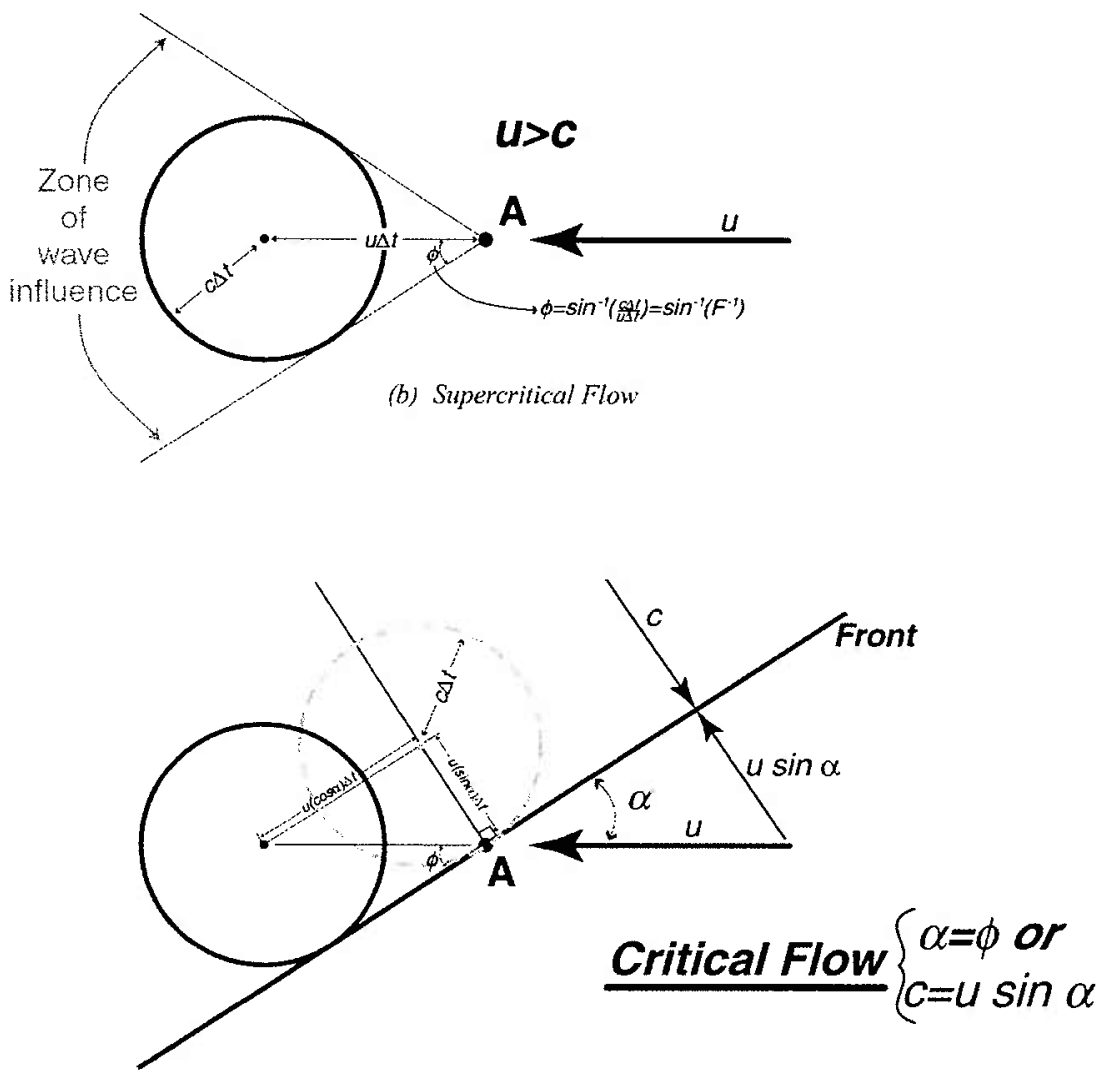

(c) Critical Flow Relative to a Two-Dimensional Front

Figure 2.10: Propogation of wave fronts for critical and supercritical flow, and critical conditions for an oblique front. In all cases a flow with velocity $u$ flows from right to left, and an instantaneous disturbance is initiated at point A. Critical flow is demonstrated in (a), where the wave speed of the fluid, $c=\sqrt{g^{\prime} h}$, is equal to $u$, and the right hand edge of the wave field forms a stationary wave at $A$, but propagates to the left elsewhere. In supercritical flow (b), all portions of the wave front move away from $\mathrm{A}$ in the same direction due to the $u>c$ condition. Here the envelope of the wave influence can be described by a Froude angle, $\phi$, as shown. In (c), it is shown how this concept of supercritical flow can represent critical flow relative to a two-dimensional front, when the Froude angle is equal to the angle of inclination between the front and the oncoming flow, $\alpha$. In this case, a Froude number calculated using the velocity component normal to the front, as demonstrated by the dashed representation of the wave front and the velocity components shown perpendicular to the front, is equal to one, as in the onedimensional case presented in (a). 
1982), and can be defined as:

$$
\phi=\sin ^{-1}\left(F^{-1}\right)
$$

Ippen (1936) derived this angle for supercritical hydraulic flows, and discussed its implications with regards to curved sections of uniform density open channel flow. Although Ippen's flow structure differs from the one presented here, the basic theory is the same: that information can only be propagated in the region centered along the direction of mean velocity and bounded by the Froude angle to either side. This region, bounded by lines tangent to the expanding wave circle at all times, represents the envelope of all characteristics emanating from the point of disturbance.

Consider a front defined by a line at an arbitrary angle, $\alpha$, with respect to the ambient flow, as shown in Figure 2.10 (c). The flow will be critical with respect to the front as long as information can be transmitted along the front, but not to the upstream side of the front. This is accomplished only when $\phi=\alpha$, so that the front must always be oriented at an angle with respect to the ambient flow equal to the Froude angle. This is equivalent to the expression $F \sin \alpha=1$, which implies that the Froude number, using the component of velocity perpendicular to the front, is critical in the traditional onedimensional sense. This interpretation is also shown in Figure 2.10 (c).

This theory was tested using the streamwise Froude numbers presented in Figure 2.8. The Froude angle was calculated for each Froude number evaluated near the observed front. These angles are shown graphically in Figure 2.11, along with the observed front locations for each transect. The consistency of these two constraints, as shown by the approximation of the front, is strong evidence that this theory is reliable in predicting the 


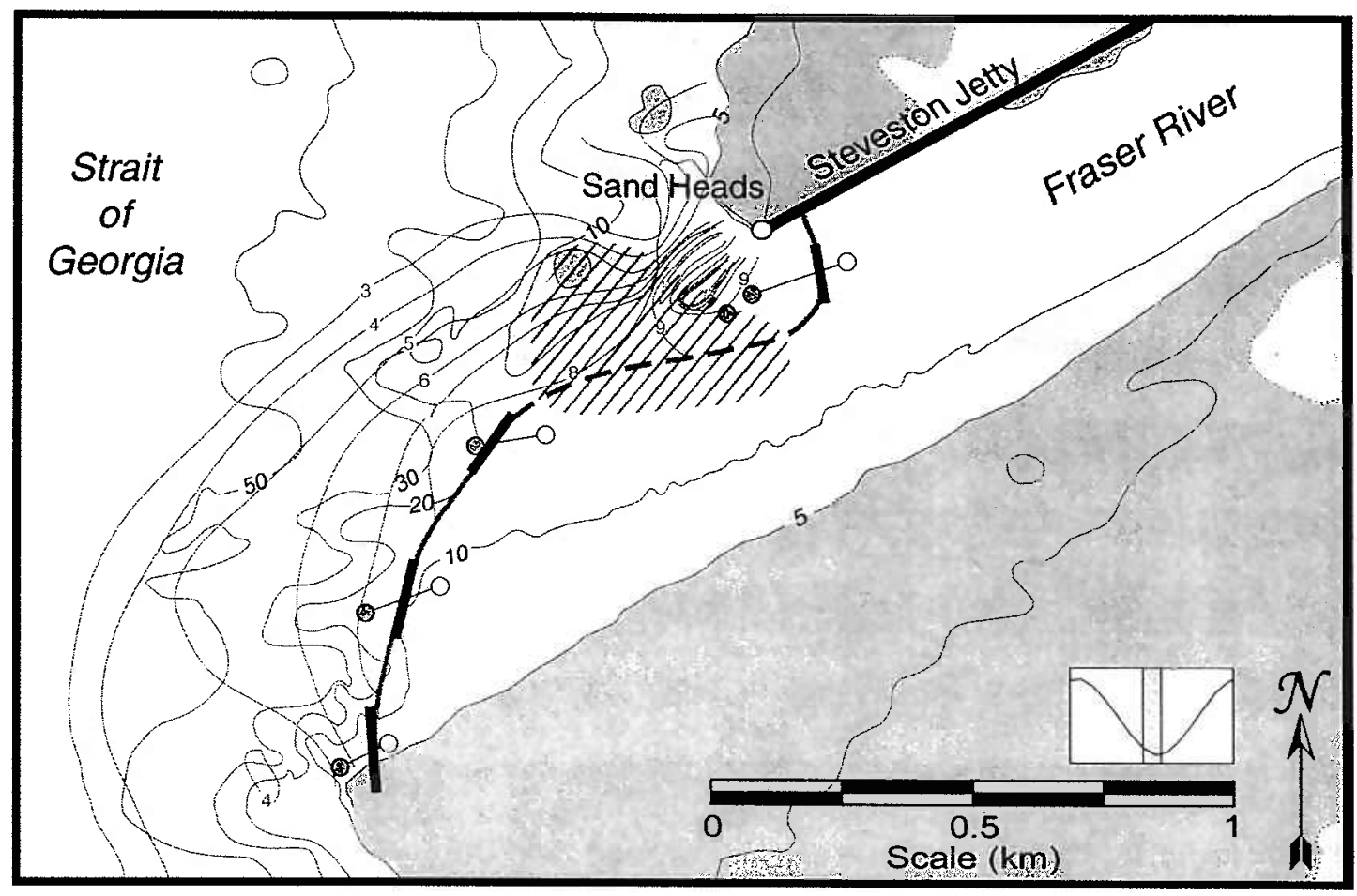

Figure 2.11: Froude angle estimates for lift-off region, calculated from the Froude numbers shown in Figure 2.8. The calculated Froude angles are shown as the bold black line segments. These segments are only shown in the vicinity of the 14 psu front, as identified by the open and closed circles, similar to Figure 2.7. The bold gray line represents the front, and meets the constraints of both the observed front location, and the Froude angle at all points. The hatched area, and dashed portion of the front, represent a region where the front is not well constrained by the available data. The front in this region may not be well defined, and dominated more by mixing processes and viscous effects than Froude number dynamics. Contours represent the depth of the $14 \mathrm{psu}$ isohaline surface. 
location and control of the front. The gray hatched area in Figure 2.11 represents a region where the location of the front is poorly constrained by the available data, and where the front may not be hydraulically controlled. The dynamics of this portion of the front are discussed in more detail later in the chapter.

\subsubsection{Expansion Control vs. Bottom Control}

Two bathymetric features are present in the Fraser liftoff zone that may provide the controlling influence on the location of the front were introduced in the previous section: the end of the lateral constraint at Sand Heads, allowing lateral expansion of flow to the north, and the presence of the bathymetric break allowing rapid expansion of flow in the vertical. Because the transmittal of information from any point is constrained by the Froude angle, the presence of the expansion at Sand Heads can only influence the location of the front to the extent that it lies within a region bounded by a path emanating from Sand Heads, and defined by the local Froude angle at all points. An approximation of this path is shown in Figure 2.12, which indicates that the Froude angle is large enough to encompass the majority of the frontal region within the zone of transmitted information. The influence of the local bathymetry, affects the entire front, so that the majority of the front, particularly the region south of the hatched area in Figure 2.11 could be influenced by either the expansion, or the rapid increase in depth.

As described above, the Froude number ultimately controls the position of the front, but changes in the Froude number are affected by both the expansion and bathymetric influences, through flow deceleration and adjustment of the layer depth. The relative importance of these two mechanisms to the value of the local Froude number must vary 


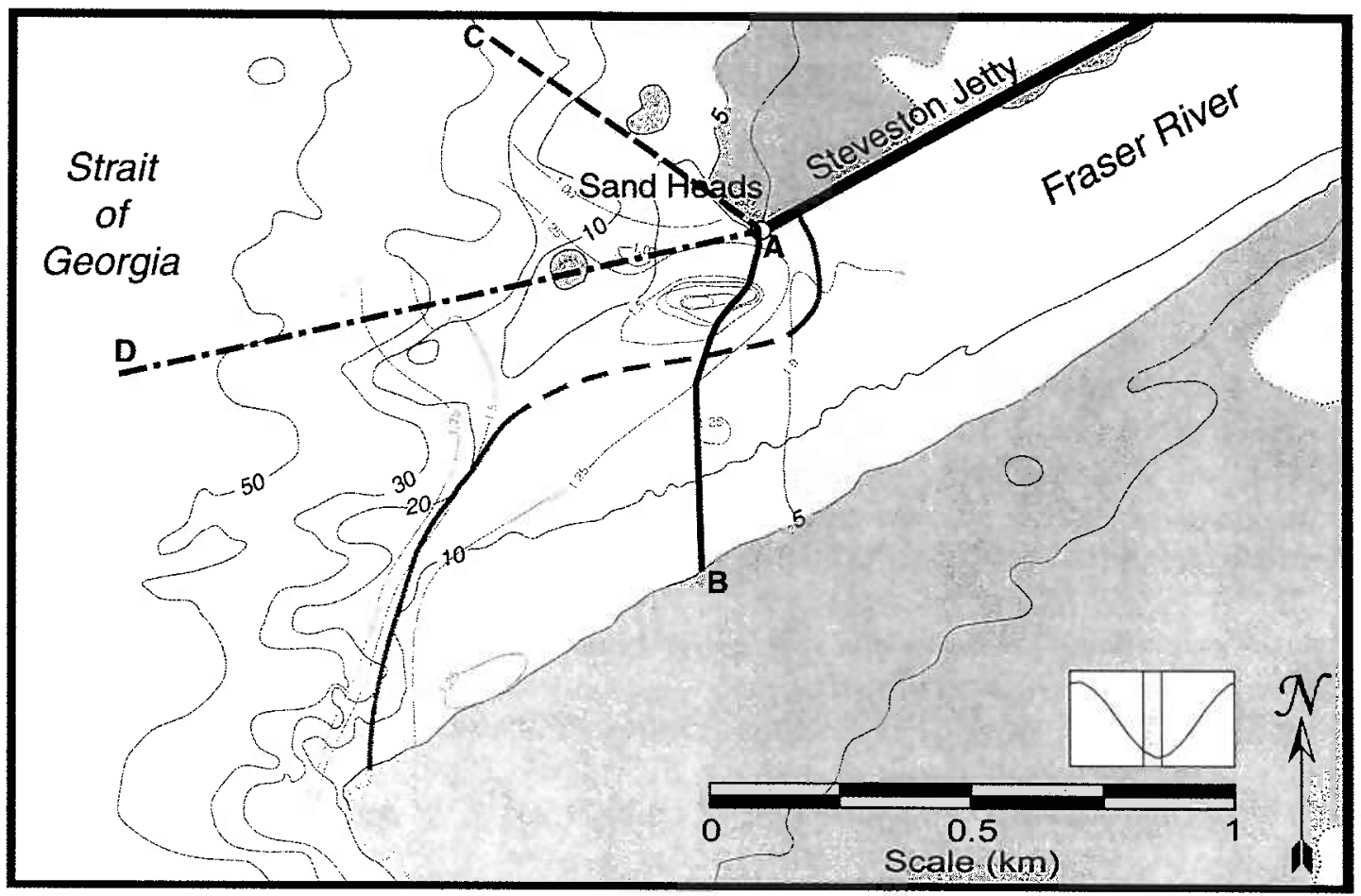

Figure 2.12: Path of information transfer from Sand Heads across the channel (AB), defined at each point by the local Froude angle. The local Froude number, and approximate location of the $14 \mathrm{psu}$ front are also shown. Lines $\mathrm{AC}$ and $\mathrm{AD}$ represent theoretical turning angles for the flow as defined by an inviscid Prandtl-Meyer expansion fan around Sand Heads. Line AC represents the maximum turning angle (i.e., $F_{1} \rightarrow \square$ ), and line $A D$ represents the angle associated with a Froude number equal to 2 , which is the point at which the inviscid assumption may break down. Streamlines, as shown in Figure 2.4 , do not turn northward to the extent of line $\mathrm{AC}$, and are more consistent with line $\mathrm{AD}$. This suggests that turbulent mixing processes may play an important role in the lateral expansion of the plume. 
with position across the channel. The front that exists landward of the line A-B shown in Figure 2.12 is controlled by bathymetric influences, as no information about the expansion can be transmitted to the region. The remainder of the front is influenced by both mechanisms.

\subsection{Kinematics of the Salt Supply}

A series of control-volume salt flux calculations were performed to investigate the kinematics of the lift-off zone. As shown in Figure 2.7, the location of the salt front expresses significant cross-channel variability. In an attempt to capture some of this variability within the salt flux calculations, control volumes were established along the northern, central, and southern portions of the lift-off region, as delineated in Figure 2.13. The northern control volume was further subdivided due to significant variability along the northern edge, particularly the dynamics associated with the scour trench in the immediate vicinity of Sand Heads. The boundaries of the resulting control volumes (IA, IB, II and III) are aligned parallel to and normal to the upper layer streamlines.

A schematic control volume, identifying the various fluxes included in the analysis is shown in Figure 2.14. Fluxes were separated into streamwise and normal orientations, and then further subdivided by geographic direction and direction relative to the control volume (i.e., in or out). A flux was calculated for each grid point on the control volume boundary, identified by a streamwise coordinate, a normal coordinate and a depth coordinate, as the product of the local salinity, the velocity perpendicular to the control volume boundary, and an area based on the resolution of the gridded data. Data from the first set of passes on June 30 were interpolated onto the streamwise-normal coordinate 


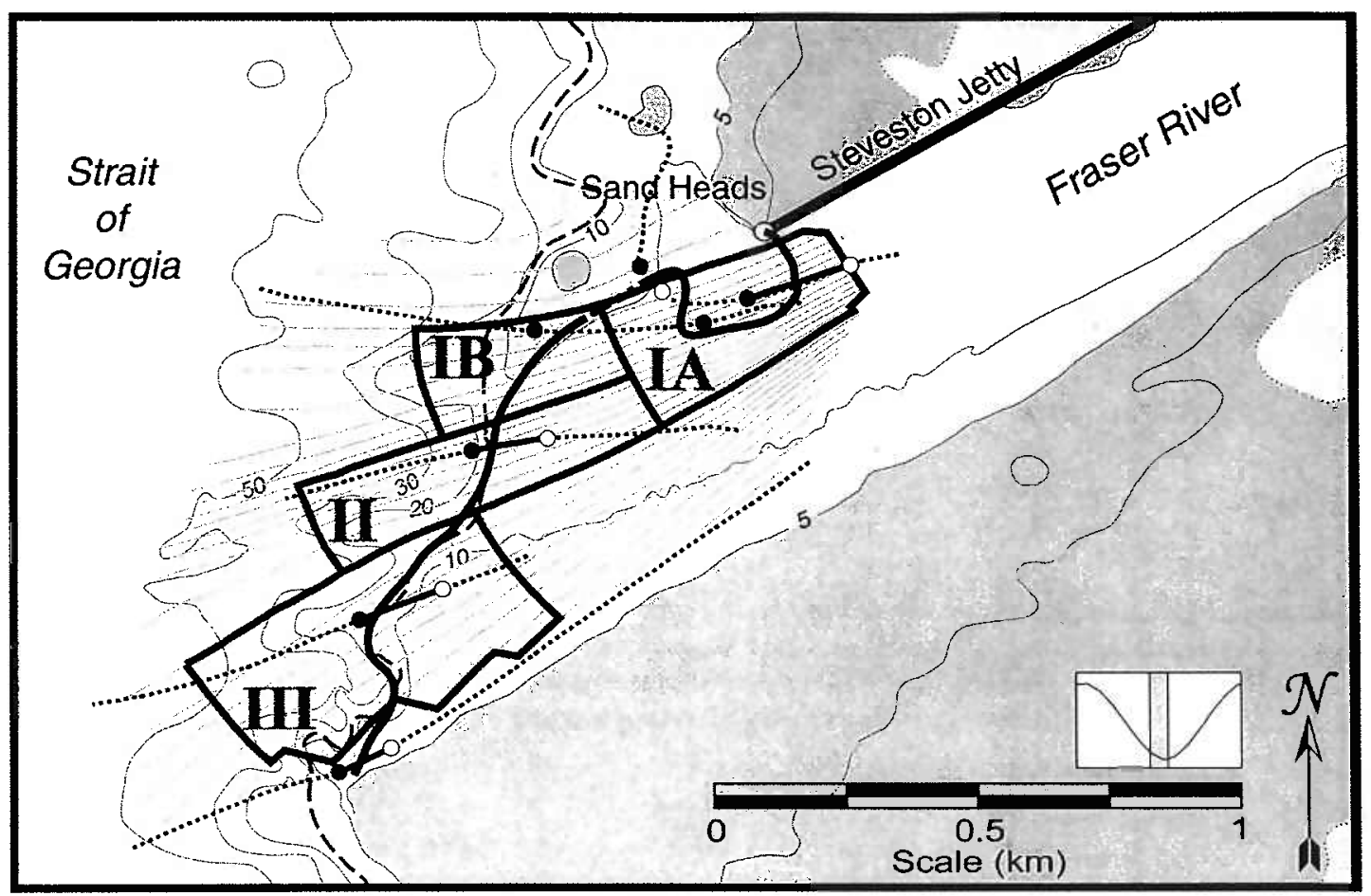

Figure 2.13: Zones for control volume salt balance analysis. Zones are arranged with boundaries coincident with streamlines and normals. The lift-off plume is divided into three regions, representative of the northern portion (I), the central portion (II), and the southern portion (III). Zone I is further subdivided to segregate the unique dynamics occurring in the vicinity of Sand Heads. An approximation of the frontal location is also shown in the figure, based on the data (filled and open circles, as in Figure 2.7), and knowledge of the local bathymetry. 


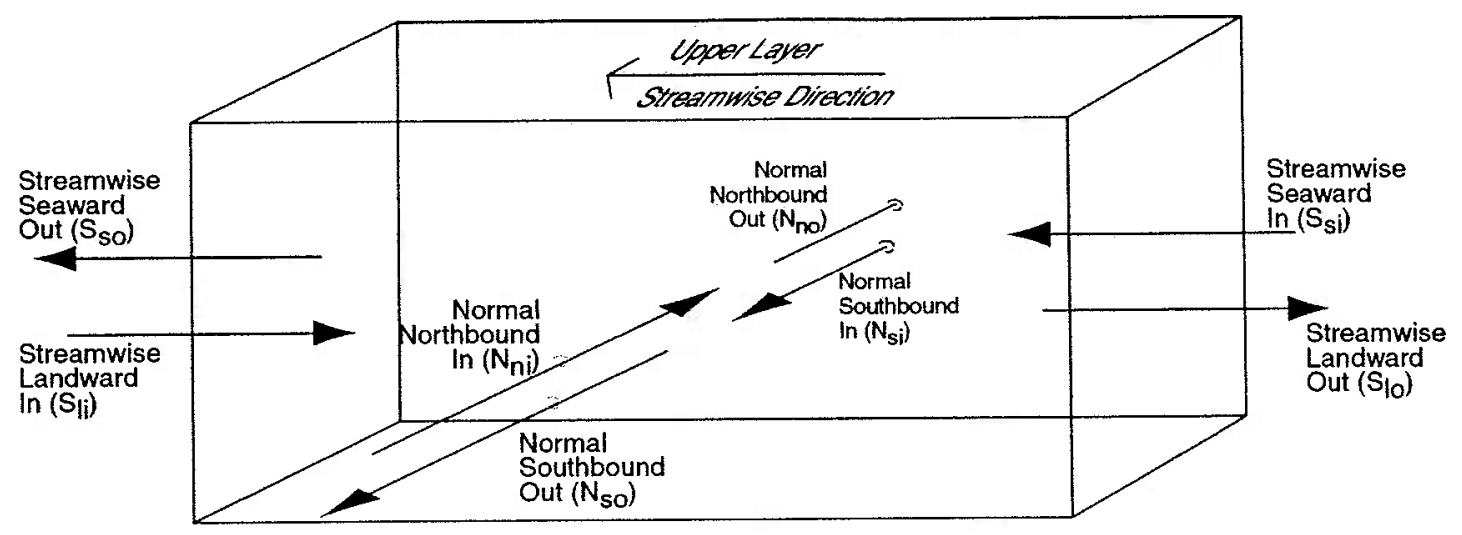

Figure 2.14: Definition sketch for control-volume salt balance analysis. Salt fluxes are considered normal or streamwise fluxes depending on which boundary of the control volume they cross. Further classification relates to direction (landward or seaward for streamwise fluxes; northbound or southbound for normal fluxes), and whether the flux is directed into or out of the control volume.

\begin{tabular}{|c|c|c|c|c|c|c|c|c|c|c|}
\hline \multirow[b]{3}{*}{ Zone } & \multicolumn{4}{|c|}{ Streamwise Flux (\%) } & \multicolumn{4}{|c|}{ Normal Flux (\%) } & \multirow{3}{*}{$\begin{array}{c}\text { Total } \\
\text { Outward } \\
\text { Directed } \\
\text { Flux } \\
\text { (psu m} / \mathrm{s} \text { ) }\end{array}$} & \multirow[b]{3}{*}{$\begin{array}{c}\text { Error } \\
(1-\operatorname{In} / O u t) \\
\text { as } \% \\
\end{array}$} \\
\hline & \multicolumn{2}{|c|}{ Seaward } & \multicolumn{2}{|c|}{ Landward } & \multicolumn{2}{|c|}{ Southbound } & \multicolumn{2}{|c|}{ Northbound } & & \\
\hline & $\begin{array}{c}\text { Out } \\
\text { (W) } \\
\mathrm{S}_{\mathrm{so}}\end{array}$ & $\begin{array}{l}\text { In } \\
\text { (E) } \\
\mathrm{S}_{\mathrm{si}}\end{array}$ & $\begin{array}{c}\text { Out } \\
(\mathrm{W}) \\
\mathrm{S}_{\mathrm{lo}}\end{array}$ & $\begin{array}{l}\text { In } \\
\text { (E) } \\
\mathrm{S}_{\mathrm{ij}}\end{array}$ & $\begin{array}{l}\text { Out } \\
\text { (S) } \\
\mathrm{N}_{\text {so }}\end{array}$ & $\begin{array}{c}\text { In } \\
(\mathrm{N}) \\
\mathrm{N}_{\mathrm{si}}\end{array}$ & $\begin{array}{l}\text { Out } \\
\text { (N) } \\
N_{n o}\end{array}$ & $\begin{array}{l}\text { In } \\
(S) \\
N_{n i} \\
\end{array}$ & & \\
\hline IA & 93 & 1 & 0 & 0 & 0 & 41 & 7 & 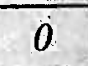 & 20,700 & 58 \\
\hline IB & 83 & 50 & 0 & 0 & 14 & 54 & 3 & 11 & 31,900 & -15 \\
\hline II & 70 & 11 & 0 & 3 & 19 & 67 & 11 & 1 & 40,600 & 17 \\
\hline III & 94 & 8 & 0 & 0 & 5 & 79 & 1 & 0 & 95,600 & 13 \\
\hline
\end{tabular}

Table 2.1: Contributions of each of the flux components shown in Figure 2.14 for the four controlvolume zones of Figure 2.13. Input and output components are identified by the subscripts shown on Figure 2.14, with the face of the control volume through which the flow is passing identified in parenthesis (i.e., east, west, north, south). Contributions are expressed as percentages of total outward directed flux, the value of which is also indicated. Closure error for each zone is shown as a percentage in the last column. 
system, and used for calculation of fluxes. These incremental fluxes were categorized by direction, as described in Figure 2.14, and summed across the entire control volume boundary.

The results of this process are shown on Table 2.1. The outbound flux (non-shaded columns) is dominated primarily by the streamwise seaward component, indicating that salt mixed within the lift-off region is being carried seaward within the plume. This is consistent with the classic two-dimensional model of estuarine circulation. The inbound flux (shaded columns) is not consistent with such a two-dimensional model, however, as the majority of salt enters the control volume in the normal southbound direction, with almost no salt entering in the streamwise landward direction. Thus, the flow supplying salt to the plume is not an estuarine circulation, but rather is a lateral inflow.

This pattern of salt entering from the north and exiting within the plume is consistent across the region, as evidenced by similar results for all four control volumes. Variations from the dominance of the normal southbound and streamwise seaward flux components are due to local effects within individual control volumes. For instance, in control volume IB, a significant fraction of the incoming salt is oriented in the streamwise seaward direction, an artifact of being directly downstream of control volume IA. A minor, but significant, component of salt exits control volumes IB, II and III in the normal southbound direction, which represents a portion of the salt supply for the region immediately to the south. This salt passes through the control volume at depth and is relatively unaffected by mixing processes higher in the water column. 
These results show that lateral input is a key component of the salt balance, and indicate the importance of cross-channel processes within the estuarine framework. This lateral delivery of salt is accomplished through a sustained velocity component in the normal direction.

\subsubsection{Distribution of Salt Flux in Salinity and Vertical Space}

The control volume approach identified that most of the salt entering the plume is advected from the north. The importance of this influx to the general dynamics of the near-field plume is dependent on whether the influx of salt is passively advected seaward, or whether the incoming flux is diluted through vertical or lateral mixing processes. These issues can be addressed by evaluating the distribution of the incoming salt with respect to both salinity and depth. To accomplish this, all boundary fluxes associated with the control volume were binned into appropriate salinity and depth bins, maintaining the directions defined in Figure 2.14, and summed.

Figures 2.15 and 2.16 show the distribution of salt flux in salinity coordinates and depth coordinates, respectively. The figures show the net streamwise flux $\left(\mathrm{S}_{\mathrm{si}}+\mathrm{S}_{\mathrm{li}}-\mathrm{S}_{\mathrm{so}}\right.$ $\left.-S_{\mathrm{lo}}\right)$ and normal flux $\left(\mathrm{N}_{\mathrm{si}}+\mathrm{N}_{\mathrm{ni}}-\mathrm{N}_{\mathrm{so}}-\mathrm{N}_{\mathrm{no}}\right)$, with respect to direction into or out of the control volume, normalized by the total outward directed flux for each zone. In Figure 2.15 , it can be seen that salt enters the control volume at significantly higher salinities than it exits in all four cases, implying that significant dilution through mixing is occurring within the lift-off zone. The change of the center of mass of salt within the control volume, with respect to salinity space, is shown in the third column of Table 2.2 . These results suggest that vertical entrainment processes are a significant component of 

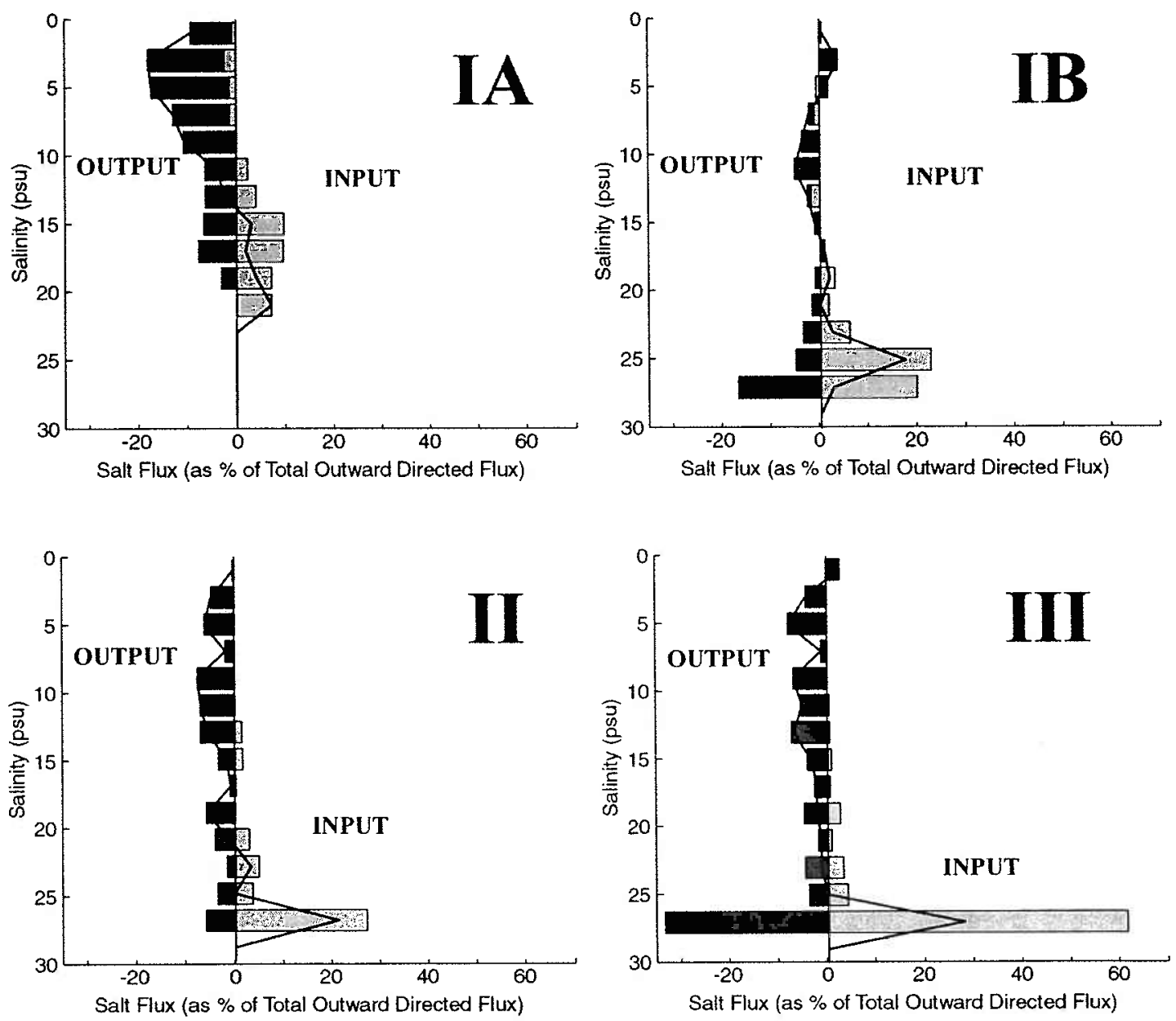

Figure 2.15: Distribution of salt flux relative to salinity for the salt flux calculation zones identified in Figure 2.13. Positive values indicate flux into the control volume, and negative values flux out of the control volume. Fluxes are aggregated into 2 psu wide bins, and normalized by the total outward directed flux for each zone (as shown on Table 1). Shading indicates alignment of flux: light shading represents normal fluxes, with dark shading representing flux in the streamwise direction. Values shown represent net fluxes in the streamwise and normal directions (i.e., streamwise flux $=\mathrm{S}_{\mathrm{si}}+\mathrm{S}_{\mathrm{ij}}-$ $\mathrm{S}_{\mathrm{so}}-\mathrm{S}_{\mathrm{lo}}$; normal flux $=\mathrm{N}_{\mathrm{si}}+\mathrm{N}_{\mathrm{ni}}-\mathrm{N}_{\mathrm{so}}-\mathrm{N}_{\mathrm{no}}$ ), which precludes the values shown from summing to $100 \%$. The black line connects the overall net flux associated with each salinity value. 

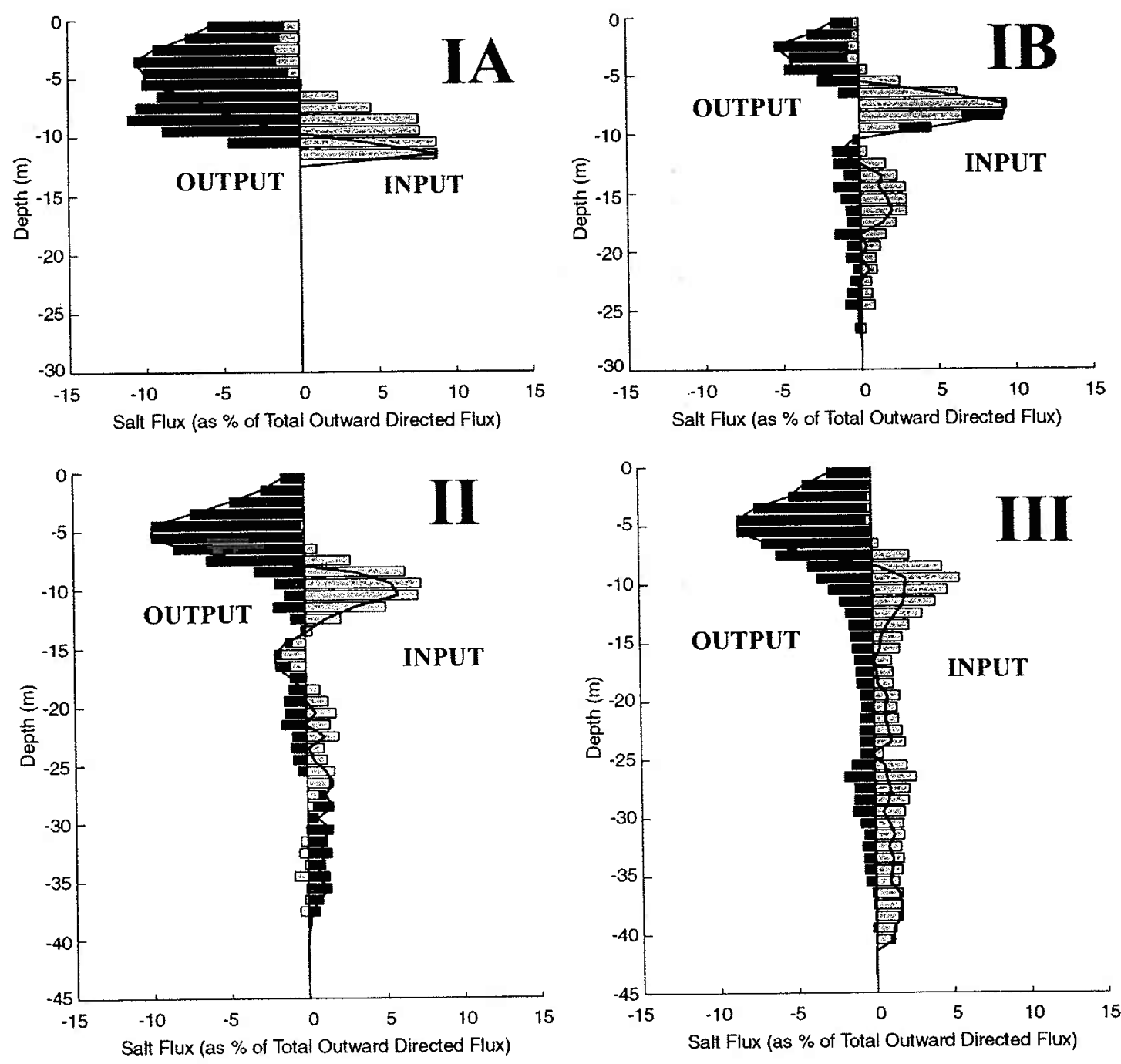

Figure 2.16: Distribution of salt flux relative to depth for salt flux calculation zones. Legend and normalization scheme are the same as Figure 2.15. 


\begin{tabular}{|c|c|c|c|c|}
\hline Zone & $\begin{array}{l}\text { Mean Salinity of } \\
\text { Input Salt Flux } \\
\text { (psu) }\end{array}$ & $\begin{array}{c}\text { Percentage of } \\
\text { Input Salt Greater } \\
\text { Than } 20 \mathrm{psu}\end{array}$ & $\begin{array}{l}\mathrm{OUT}_{\mathrm{s}}-\mathrm{IN}_{\mathrm{s}} \\
\quad(\mathrm{psu})\end{array}$ & $\begin{array}{l}\mathrm{OUT}_{\mathbf{z}}-\mathbb{I N}_{\mathrm{z}} \\
(\mathrm{m})\end{array}$ \\
\hline IA & 16.8 & 18 & -8.8 & 4.0 \\
\hline IB & 21.9 & 75 & -2.6 & 2.3 \\
\hline II & 24.1 & 82 & -10.1 & 8.7 \\
\hline III & 25.3 & 89 & -7.0 & 10.5 \\
\hline
\end{tabular}

Table 2.2: Salt input/output distribution statistics, gleaned from data in Figures 2.15 and 2.16. OUT and IN are the centers of mass of the output salt and input salt, respectively. The subscript $s$ indicates salinity space (psu) and the subscript $\mathrm{z}$ indicates vertical space $(\mathrm{m})$.

the local dynamics, particularly in Zones IA, II and III. The distribution in Zone IB is impacted by the large influx of mixed water entering in the streamwise direction.

The complicating influences of mixed water influx are confined primarily to the northern edge of the plume. The first two columns of Table 2.2 show the pronounced increase in the salinity of the input salt flux as one moves along the front southward from Sand Heads (Zone IA to III). The input salt flux to Zone IA consists exclusively of mixed water, with less than $20 \%$ of the input flux at salinities greater than 20 psu. In contrast, the input flux in Zone III consists almost exclusively of unmixed Georgia Strait water, with a mean salinity well in excess of $20 \mathrm{psu}$. Zone III exhibits the most welldefined two-layer entrainment regime, where two water masses of decidedly different salinity are interacting. The kinematics in Zone IA are more complex, with previously mixed water of intermediate salinity feeding mixing processes, and no clear density distinction between the two major water masses. 
The distribution in Figure 2.16 clearly indicates that the center of mass of the output salt is situated higher in the water column than the center of mass of the input salt. This can also be seen in the last column of Table 2.2, which indicates a more significant vertical increase along the southern edge of the channel, where the source salt is relatively unmixed Georgia Strait water. The northern portion of the front has limited access to deep water and relies on the shallower source of partially mixed water residing north of Sand Heads for dilution of the discharging fresh river water.

The along-channel sections presented in Figure 2.4 identify a zone of high southbound directed velocities just below the pycnocline across the entire region. This current, with maximum velocities approaching $0.5 \mathrm{~ms}^{-1}$, is consistent with the distributions shown in Figure 2.15, where strong velocities at high salinities result in large fluxes. It is also consistent with Figure 2.16, where the peaks in inbound salt flux tend to occur high in the water column, just below the region of high seaward velocity within the plume.

The lateral flux of salt identified as the primary source of salt supplying the entrainment processes within the lift-off zone is driven by this southward-directed flow, which is part of a tidal motion in the Strait of Georgia. Because the steady-state lift-off period occurs during the ebbing tide, the receiving waters of the Strait of Georgia are ebbing and directed southward, towards the Strait of Juan de Fuca and the open ocean. A model of tidal velocities in the Strait of Georgia, absent any influence from the Fraser River, predicts southward-directed velocities on the order of 20 to $40 \mathrm{~cm} \mathrm{~s}^{-1}$ in the vicinity of Sand Heads during the ebb tide (Crean et al., 1988). These model results are 
consistent with the observations shown in Figure 2.4, and indicate that the ambient cross flow of the receiving waters plays a crucial role in defining the structure of the lift-off region.

\subsubsection{Potential Energy Changes Within the Lift-Off Zone}

The distributions shown in Figure 2.15 indicate that mixing plays a dominant role within the lift-off zone, as evidenced by the apparent dilution of inbound salt flux. The distributions in Figure 2.16 further suggest that the dilution is the product of vertical mixing, given the difference in the vertical center of mass between the outbound and inbound flux. A more rigorous examination of the potential energy change within the control volumes is required, however, in order to demonstrate that vertical mixing is clearly dominant over lateral mixing. For example, lateral mixing, particularly along the inclined side boundaries of a plume detaching as described by Adams and Stoltzenbach (1977), could be responsible for the dilution of incoming flux. However, in the absence of vertical mixing and entrainment there would be no change in the center of mass (or potential energy) of the salt, and any vertical motion would require a separate advective process.

An upper bound estimate of the potential energy increase that could be accomplished in the absence of vertical entrainment was accomplished with the Zone III data by focusing on the potential energy increase that would be required through advection. For this analysis, a salinity profile representative of the deepest observation of each particular salinity value was compared with the observed output salinity profile. The potential energy increase necessary to transform the first salinity profile into the second, given the 
observed fluxes, is only approximately one-third of the observed increase. This implies that salt must travel further in the vertical than possible through only advective processes, and that mixing across isohalines must be required.

In contrast, a mean salinity profile for Zone III was used to generate an estimate of the potential energy increase that would be associated strictly with vertical mixing and entrainment. For this simplified estimate it is assumed that the salinity profile does not change between the input and output, but the distribution of flux with respect to salinity does vary, as indicated by the distributions in Figure 2.15. This method yielded an energy change approximately $30 \%$ greater than the observed change. Using these two ratios, and distributing the total observed salt flux between vertical and lateral mixing in order to match the observed energy change (i.e., $\frac{1}{3} M_{L}+\frac{4}{3}\left(1-M_{L}\right)=1$, where $M_{L}$ is the fraction of diapycnal salt flux accomplished through lateral mixing) suggests that, as an upper limit, approximately one third of the observed salt flux could be occurring through lateral processes.

This exercise confirms that the majority of the salt related potential energy changes observed within the lift-off zone are the result of vertical mixing. At most, only approximately a third, and more likely much less, of the observed potential energy increase could be due to a combination of lateral mixing followed by vertical advection. If lateral mixing were dominant a much steeper streamwise baroclinic gradient would be required in order to match the observed potential energy increase.

As discussed in Section 2.1, the aspect ratio of the discharge, i.e., the ratio of depth to width, is important in determining the relative importance of lateral mixing processes. 
In the Fraser the aspect ratio is quite small, on the order of $10^{-2}$. Together with the results of the potential energy comparisons, this geometry supports the conclusion that lateral mixing processes are not the dominant mixing mechanism in the lift-off zone. Similar potential energy comparisons for flows with much larger aspect ratios should indicate that lateral processes would be more important.

It is possible that lateral impacts may play an enhanced role near the northern edge of the plume, where there is a lateral intrusion of salt at depth and a lift-off characterized by side impingement. A local aspect ratio in this region, estimated from the slope of the isohalines shown in cross section $\mathrm{D}^{-\mathrm{D}^{\prime}}$ of Figure 2.5 , is approximately a factor of two larger than the overall aspect ratio of the plume, but still considerably smaller than the aspect ratios associated with the lift-off experiments of Adams and Stolzenbach (1977).

\subsection{Cross Channel Dynamics}

The previous sections have indicated that the spreading of the plume is an important aspect of the dynamics. In the immediate vicinity of Sand Heads, the expansion of flow to the north can be considered analogous to the Prandtl-Meyer expansion of a compressible gas, as discussed by Garvine (1982), and Baines (1995, p. 88). Based on simple wave theory, the Froude number should be constant along radial lines of wave propagation emanating from the point of transition (in this case Sand Heads). The Froude number increases with the size of the angle between the radial and the initial flow direction. Radials are crossed by streamlines as they turn due to the expansion, so that 
the Froude number along a streamline monotonically increases. Following Garvine (1982), the Prandtl-Meyer function for hydraulics can be written as:

$$
v=3^{\frac{1}{2}} \tan ^{-1}\left[3^{-\frac{1}{2}}\left(F^{2}-1\right)^{\frac{1}{2}}\right]-\tan ^{-1}\left[\left(F^{2}-1\right)^{\frac{1}{2}}\right]
$$

where $F$ is the local Froude number. This angle between the initial flow direction, and the radial associated with a specific Froude number is equal to the difference between corresponding values of the Prandtl-Meyer function

$$
\theta=v(F)-v\left(F_{e}\right)
$$

where $F_{e}$ is the initial Froude number of the flow at the expansion.

A maximum turning angle can be associated with a local Froude number theoretically equal to infinity (e.g., Chapman and Walker, 1971, pp 369-391). Because the Froude number of a real flow cannot equal infinity, streamlines cannot cross this radial, and it defines an outer limit for inviscid expansion. Using (2.3), with $F \rightarrow \infty$, and an initial Froude number $\left(F_{e}\right)$ of 1.2 , the maximum turning angle is approximately $63^{\circ}$. This angle is shown as line segment $\mathrm{AC}$ on Figure 2.12. The radial for an angle, $\theta$, corresponding to $F=2$ is also shown on Figure 2.12 as line segment AD. At Froude numbers higher than approximately a value of 2 , the presence of intense mixing may invalidate the inviscid assumption. Comparison of the streamlines in Figure 2.3 to the Prandtl-Meyer angles in Figure 2.12 suggest that plume expansion is more consistent with the $F=2$ assumption, and that interfacial mixing must play a significant role in setting the rate of plume expansion. 
An evaluation of the important terms in the lateral momentum balance can shed further light on the dynamics responsible for setting the observed expansion rate, although the resolution of the present data set in the cross-channel direction is not sufficient to completely resolve the balance.

Upper and lower layer transverse momentum equations can be written following Geyer (1993). By subtracting the equation of the lower layer from that of the upper layer, the unknown barotropic term can be removed from the analysis:

$$
\frac{u_{s 1}^{2}}{R}-\frac{u_{s 2}^{2}}{R}+u_{s 2} \frac{\partial u_{n 2}}{\partial s}+u_{n 2} \frac{\partial u_{n 2}}{\partial n}-f u_{s 1}+f u_{s 2}=-g^{\prime} \frac{\partial h_{i}}{\partial n}-\frac{\tau_{b}}{\rho h_{2}}+\frac{\tau_{i}}{\rho} \frac{\left(h_{1}+h_{2}\right)}{h_{1} h_{2}}
$$

Here $u$ represents velocity, with the subscripts $s, n, 1$, and 2 referring to the streamwise direction (referenced to the upper layer), the normal direction, the upper layer, and the lower layer, respectively. The Coriolis parameter is represented by $f, g^{\prime}=g(\Delta \rho) \rho_{\circ}^{-1}$ is a reduced gravity based on the density difference between the upper and lower layers, $R$ is the radius of curvature for the upper layer streamlines (clockwise positive), $\tau_{b}$ and $\tau_{i}$ represent bottom and interfacial stress, respectively, $h_{i}$ represents the height of the layer interface above an arbitrary datum, and $h_{1}$ and $h_{2}$ represent the individual layer depths. In this formulation the frame of reference is established so that $u_{n l}$ is zero everywhere. The curvature terms (represented by the first two terms in the equation) represent the centripetal acceleration of the fluid.

Estimation of the terms in (2.4) was conducted using data from the five transects interpolated onto the streamwise-normal grid. To estimate the interfacial stress term, a drag coefficient on the order of $5 \times 10^{-4}$ was used, the derivation of which is described in 
more detail in the next chapter. The exercise indicated that the most dominant terms in (2.4) are the upper layer curvature term, $\frac{u_{s 1}^{2}}{R}$, and the baroclinic term, $g^{\prime} \frac{\partial h_{i}}{\partial n}$.

Although the Coriolis term is important at larger scales across the far field of the plume (e.g., Fong, 1998), the length scales of the lift-off zone are too small for the term to have a significant impact in the near-field region. As a result, the Coriolis term in Equation (2.4) is roughly an order of magnitude smaller than the curvature term, and the near field region can be considered in a non-rotating reference frame.

There are at least two regions where the transverse baroclinic gradient is well constrained in the cross-channel transects shown in Figure (2.5): cross section C-C' between cruise tracks 3 and 4, and cross section B-B' between cruise tracks 2 and 3 . Assuming a simple balance between curvature and baroclinicity, as suggested above, would require a radius of curvature on the order of 1 to $2 \mathrm{~km}$. A radius of this magnitude is consistent with observed curvature near the northern end of cross section C-C', near cruise track 4, but does not appear representative of the remainder of these two regions. The lateral resolution of the present data set may not be sufficient to accurately measure localized radii of curvature, which may be affected by variability at small scales due to local bathymetry. Despite these limitations, it is likely that the balance between curvature and baroclinicity is adequate to describe the cross channel dynamics of the near-field plume. 


\subsection{Implications of Froude Angle Control and Local Kinematics}

The analyses summarized in Figure 2.11 suggest that flow is controlled in the lift-off region not by the Froude number, but by the Froude angle. This results in a significantly relaxed constraint on front position and orientation, allowing a steady front to occur under supercritical conditions. This relaxed constraint has significant implications for the dynamics associated with estuarine fronts in many diverse situations.

An important aspect of the use of the Froude angle concept is that no universal critical condition can be formulated to describe the position of the front at a point, or to predict the location of the front along a 2-D transect. However, when attempting to identify the location of a front using this concept, an additional constraint exists. The Froude angle concept requires that the front be continuous, because a wave emanating from any point on the front must propagate along the front as it expands. In the salt front analogy with nonzero viscosity (the physically realistic case), it is the transport of salt that is important. A flux of salt is required along the froude angle defined path in order to replenish salt lost to entrainment and maintain the stability of the front. At the upstream endpoint of the front, there can be no along Froude-angle salt flux from an upstream position, implying that the front must be locally critical at the endpoint (i.e., $G=F_{1}=1$ ), and perpendicular to the oncoming flow with $\alpha=90^{\circ}$. This condition provides the needed constraint, anchoring the front at a particular location, and allowing the shape of the front to be predicted by propagating a hypothetical wave front across the channel following the direction of the local Froude angle. The endpoint of a density front could be forced by the presence of a wall or other boundary in a confined channel. 
Alternatively, local cross channel symmetry centered on a bathymetric depression could have a similar effect. It is unclear if this constraint would exist for the invisicid case, or if it is possible for the front to be oblique to the oncoming flow along its entire length. In the latter case the location of the front could not be controlled without an additional constraint.

Consideration of the Froude angle represents a significant extension of the twodimensional theory presented by Farmer and Armi (1986), and Armi and Farmer (1986). The classic two-dimensional theory represents a limiting case for the Froude angle concept, characterized by a front perpendicular to the oncoming flow, $\alpha=90^{\circ}$. A second limiting case would occur at $\alpha=0^{\circ}$, with the front parallel to the oncoming flow. Because this case requires that the Froude number must go to infinity at the front for a critical condition to exist, no critical condition can be supported. The resulting front cannot be balanced by inviscid dynamics, and would be characterized by weaker gradients and transient fluctuations.

Most of these dynamic implications of the Froude angle can be observed in Figure 2.11. At its northern endpoint, the front is consistent with the limiting case of $\alpha=90^{\circ}$, intersecting the Steveston Jetty perpendicular to the channel with a Froude number approximately equal to one. Following the front southward, the shape of the front is defined by the local Froude angle, but it does not take a shape similar to the line $\mathrm{AB}$ shown in Figure 2.12, which is the Froude angle defined path emanating from Sand Heads. Instead the front must approach the limiting case of $\alpha=0^{\circ}$, running approximately parallel to the channel through the hatched region in Figure 2.11 until it 
once again becomes hydraulically controlled and stable near the bathymetric break. An explanation for this discrepency can be found by once again examining the Froude number contours of Figure 2.8. The broad region of the channel adjacent to the hatched region is characterized by relatively constant Froude numbers. A front may stabilize in this region briefly at the beginning of the lift-off period as it retreats from the channel. However, any perturbation in flow rate that briefly increases the local Froude number would drive the front seaward. Upon relaxation of the increase, the salt wedge cannot return landward to its original position because conditions remain critical at the new location. In this manner, the front is ultimately pushed to the edge of the bathymetric break where there is a stronger Froude number gradient. In the case of the Fraser, this results in a break in the continuity of the controlled front, as represented by the hatched region in Figure 2.11. Because of a source of salt water supplied through the scour trench around Sand Heads, the northern portion of the front is dynamically isolated from the southern portion, and can remain stable in its original location.

The resulting shape of the front within the hatched region must be uncontrolled at least across part of its length, and ultimately stabilized only by the existence of controlled fronts to either side. One possibility is that the front is characterized by a large fold as suggested in Figure 2.7. In this case, the most seaward portion of the front within the hatched region would likely be controlled by a critical Froude angle, although control of the landward portion (a "reverse" front, as the bottom layer would disappear as one moves in the streamwise direction) would not be characterized by the same dynamics. Alternatively, an unsteady transport of salt from the hatched region towards the south 
may be triggered in order establish the front along an unbroken Froude angle path. However, any salt transported south of the hatched region will ultimately be flushed seaward by the mechanism described above, resulting in an unstable "fluttering" front.

The concept of Froude angle control should be applicable in many different hydraulic control scenarios. Garvine (1982), developed a model for plume expansion, which incorporated a similar Froude angle dependence for defining the surface fronts of buoyant plumes. The model was used to predict the location and orientation of the surface front with respect to an ambient crossflow, and explained the structure of the Connecticut River plume front. Bottom-attached fronts with similar orientation to the front observed at the mouth of the Fraser have been predicted analytically and observed in laboratory experiments for buoyant jets discharged over a uniformly sloping bottom (Adams and Stolzenbach, 1977). The bottom attached region in these cases consisted of a long and narrow triangular region, with the bottom front emanating into the jet region at an angle from the end of both channel boundaries. The basic geometry of this solution is consistent with the Froude angle theory. A similar example of Froude angle criticality has been seen within the confines of the estuarine channel in the Hudson River (New York), where angled fronts have been observed at supercritical streamwise Froude numbers (Geyer et al., 1998). These observed angled fronts are driven by the significant cross-channel variability in bathymetry within the Hudson channel.

The importance of the Froude angle may also be significant in hydraulic control situations where both layers are active, although three-dimensional observations of these phenomena are scarce. Klymak and Gregg (2001) emphasize the importance of three 
dimensional structure to the dynamics of a stratified overflow in Knight Inlet, identifiying lateral recirculations in the lee of the sill. This particular overflow has been well studied along the thalweg (Farmer and Armi, 1999), with observations that are quite consistent with the Farmer and Armi (1986) theory. However, the importance of lateral structure outside of the thalweg, as shown by Klymak and Gregg (2001), suggests that there could also be lateral implications for hydraulic control. In this case the symmetry present in the thalweg may locally force the orientation of the front towards $\alpha=90^{\circ}$,

\subsubsection{Importance of Lateral Flow}

Analysis of the salt supply components indicated that the ebbing tidal structure in the Strait of Georgia is responsible for creating a sub-pycnocline flow transverse to the streamlines of river discharge, which plays a significant role in the dynamics and kinematics of the front. The Fraser is not unique in that respect, as many rivers discharge into regions of active cross-flow, including the Connecticut (Garvine, 1974), and the Columbia (Hickey et al, 1998). Salt entrainment in the Amazon plume is also believed to be from lateral sources (Geyer and Kineke, 1995), possibly due to an ambient crossflow. At large scales, the absence of a cross-flow would affect the transport of plume waters away from the estuary mouth, resulting in the growth of a brackish buoyant layer. This layer would eventually impact the relatively "clean" dynamics of the Fraser lift-off region through re-entrainment and broadening of the pycnocline. In fact, it is the delivery of relatively unmixed Strait of Georgia water to the majority of the lift-off zone that allows a simplification of the region's dynamics. For example, salt budgets in this region can be carried out effectively in the streamwise direction, without a thorough analysis of the 
cross-channel influences, by assuming that all salt input enters at salinities equivalent to that found in the deep water observed within the two-dimensional transect. The input of salt through the re-entrainment of brackish water would confound such analyses, as the observation of brackish output water could not necessarily be attributed to mixing.

The transverse communication of information, as discussed with respect to the Froude angle concept, does not require the existence of an ambient transverse current, and it does not appear that the cross stream flow is a direct controlling factor in setting the location of the front. The location and angle of the front is established to match the conditions of the oncoming river flow, as modified exclusively by local bathymetry and channel constraints. Due to the angled nature of the front relative to the streamlines, there is a non-zero component of velocity in the along front direction which is sufficient for the transmittance of information and mass. In the absence of an ambient cross-flow, the front would likely remain in a similar position, and the salt mass required to balance entrainment processes would be drawn from deeper local sources rather than the lateral sources observed. Similarly angled fronts are observed within the laterally confined channel of the Hudson River, where no ambient cross channel flow is present (Geyer et al, 1998).

\subsection{Concluding Remarks on Lift-Off Zone Structure}

The Fraser River liftoff is a good example of highly stratified, or two-layer, arrested flow, and illustrates many aspects of estuarine fronts common to other physical settings. The steady front that forms outside the mouth of the Fraser during late ebb is controlled 
by the interplay between the sudden expansion of the channel and the local bathymetry. The alignment of the front is established in response to the local Froude angle of the flow within the channel. This importance of the Froude angle as the measure of criticality, as opposed to the Froude number itself, represents a significant three-dimensional extension of the two-dimensional theory presented by Armi and Farmer (1986) and Farmer and Armi (1986). Previous data collected from other estuaries (Wright and Coleman, 1971; Kashiwamura and Yoshida, 1978; Park et al., 1993) has tended to support the twodimensional theory, indicating that the Froude number is in the vicinity of one near the front. In most cases, these studies have not consisted of enough data to define the front in three dimensions, and researchers have been left to discuss an inherently threedimensional problem by loosely applying a two-dimensional theory to the limited data. In some cases, where the orientation of the front has been determined sufficiently to locate measurements in a velocity field normal to the front, the two-dimensional theory suffices (Huzzey, 1982). The data presented here enables the delineation of the front in three-dimensions, and shows that the Froude number is exactly unity when calculated perpendicular to the front.

Calculation of the salt budget within the lift-off zone indicates that most of the salt used to balance entrainment processes is supplied by an ambient southward-directed, tidally-driven current in the Strait of Georgia. Although this current plays an important role in the kinematics of the lift-off zone, it does not appear to be an important factor in the positioning of the front. In the absence of an ambient current, however, the 
kinematics seaward of the front would be markedly different, as more salt would be drawn from the local depths.

The location of an estuarine front, and the dynamics associated with it, are of great practical interest for many biological, geomorphological, and engineering related issues. A more detailed study of the mixing and entrainment processes local to the lift-off region will be examined in the next chapter. Combined, these issues provide a significant physical treatment of the lift-off zone, a region that has a significant impact on far-field plume characteristics, in addition to interesting local dynamics. 


\section{Chapter 3}

\section{Turbulent Energy Production and Entrainment at a Highly Stratified Estuarine Front}




\section{ABSTRACT}

Rates of turbulent kinetic energy (TKE) production and buoyancy flux in the region immediately seaward $(\sim 1 \mathrm{~km})$ of a highly stratified estuarine front at the mouth of the Fraser River (British Columbia, Canada) are calculated using a control volume approach. The calculations are based on field data obtained from shipboard instrumentation, specifically velocity data from a ship mounted acoustic Doppler current profiler (ADCP), and salinity data from a towed conductivity-temperature-depth (CTD) unit. The results allow for the calculation of mean vertical velocities in the water column, and the total vertical transport of salt and momentum. The vertical turbulent transport quantities $\left(\overline{u^{\prime} w^{\prime}}, \overline{S^{\prime} w^{\prime}}\right)$ can then be estimated as the difference between the total transport and the advective vertical transport. Estimated production is on the order of $10^{-3} \mathrm{~m}^{2} \mathrm{~s}^{-3}$, yielding a turbulent Reynolds number, $\frac{\varepsilon}{v N^{2}}$, on the order of $10^{4}$. This rate of TKE production is at the upper limit of reported values for ocean and coastal environments. Flux Richardson numbers in this highly energetic system generally range from 0.15 to 0.2 , with most mixing occurring at gradient Richardson numbers slightly less than $1 / 4$. These values compare favorably with other values in the literature that are associated with turbulence observations from regimes characterized by scales several orders of magnitude smaller than are present in the Fraser. 


\subsection{Mixing in Shear-Stratified Flows}

Many important geophysical flows are both stratified and sheared. Turbulence can be generated in these flows when the stabilizing influence of the stratification is not sufficient to damp the destabilizing effects of the velocity shear (Thorpe, 1971, 1973). The onset of turbulence in such flows can greatly enhance the vertical transport of momentum, buoyancy, and other dissolved constituents. Shear stratified flows can be found within the pycnocline in oceans, lakes, reservoirs and estuaries as well as stratified atmospheric dynamics. Most investigations concerning the nature of shear-stratified turbulence, however, are based on laboratory experiments (e.g. Ivey and Imberger, 1991), or observations from relatively low-energy geophysical regimes (e.g. Gregg, 1989). In this chapter, turbulence measurements, obtained from momentum and buoyancy budgets at the mouth of a large river, are presented as an opportunity to evaluate the nature of shear-stratified turbulence in a relatively high energy regime.

\subsubsection{Turbulent Kinetic Energy and Mixing Efficiency}

The turbulent kinetic energy (TKE) budget in a stratified flow can be written as (e.g. Osborn, 1980):

$$
\frac{D}{D t} \frac{1}{2} \overline{u_{i}^{\prime} \cdot u_{i}^{\prime}}=\left(2 v \frac{\partial}{\partial x_{j}} \overline{u_{i}^{\prime} e_{i j}}-\left(\bar{\alpha}+\alpha^{\prime}\right) u_{i}^{\prime} \frac{\partial p^{\prime}}{\partial x}\right)-\frac{\mathbf{P}}{-\overline{u_{i}^{\prime} u_{j}^{\prime}} \frac{\partial u_{i}}{\partial x_{j}}-\frac{g}{\rho_{o}} \overline{\rho^{\prime} u_{3}^{\prime}}-2 v \overline{e_{i j} e_{i j}}}
$$

where $u_{i}$ is the velocity vector, ? represents density, $p$ pressure, ? the coefficient of kinematic viscosity, $\alpha$ the specific volume, $e_{i j}$ the fluctuating strain rate, given by 
$e_{i j}=\frac{1}{2}\left(\frac{\partial u_{i}^{\prime}}{\partial x_{j}}+\frac{\partial u_{j}^{\prime}}{\partial x_{i}}\right), x_{3}$ is directed vertically upward, and primes denote fluctuating quantities or a departure from the mean quantity, which is represented by an overbar. When the turbulence field is homogenous, and in steady state, equation (3.1) reduces to a balance between the shear production (denoted by $\mathbf{P}$ in 3.1), buoyant production (B), and dissipation (e):

$$
\mathrm{P}=\mathrm{B}+\varepsilon
$$

In stably stratified flows, the buoyant production term is an energy sink, representing the conversion of kinetic energy to potential energy through the redistribution of the vertical density profile. In this context, buoyant production, also referred to as buoyancy flux, is representative of the amount of energy input into irreversible mixing, and can also be expressed in terms of a vertical turbulent eddy diffusivity for density, $K_{z}^{\rho}$ :

$$
K_{z}^{\rho}=\frac{\overline{\rho^{\prime} w^{\prime}}}{\frac{\partial \rho}{\partial z}}=\frac{B}{N^{2}}
$$

where $N=\left(\frac{-g}{\rho} \frac{\partial \rho}{\partial z}\right)^{\frac{1}{2}}$ is the buoyancy frequency. A similar eddy diffusivity for momentum, also referred to as an eddy viscosity, $K_{z}^{u}$, is often assumed equal to $K_{z}^{\rho}$, and is defined similarly as $\frac{\overline{u^{\prime} w^{\prime}}}{\frac{\partial u}{\partial z}}$. TKE not converted to potential energy through the buoyancy flux term is dissipated at viscous scales as represented by the $e$ term in (3.1). 
The flux Richardson number represents a mixing efficiency defined as the ratio of buoyancy flux to shear production, and indicates the fraction of available TKE that is converted to potential energy through vertical turbulent transport:

$$
R i_{f}=\frac{B}{P}
$$

Many studies have attempted to quantify this ratio under varying turbulent conditions. Osborn (1980) suggested a value of $R i_{f}$ equal to 0.15 based on observations of oceanic turbulence. Ivey and Imberger (1991) employed laboratory experiments to investigate the relationship between $R i_{f}$ and several turbulent parameters, concluding that the flux Richardson number should fall in a range between 0.15 and 0.2 for flows with turbulent Reynolds numbers $\left(\operatorname{Re}_{T}=\frac{\varepsilon}{v N^{2}}\right)$ greater than a value approximately equal to 10 , assuming an overturn Froude number $\left(F r_{T}=\varepsilon^{\frac{1}{3}} / g^{, \frac{1}{9}} L_{d}{ }^{\frac{1}{6}}\right.$, where $g^{\prime}$ is a reduced gravity and $L_{d}$ a centered displacement scale), equal to one. This assumption is consistent with most oceanic thermocline measurements (Gregg, 1987, Peters et al., 1988), where Kelvin-Helmholz billows are believed to be the primary mechanism of turbulence generation. Although their laboratory data was limited to $\frac{\varepsilon}{v N^{2}}$ values less than 20 , Ivey and Imberger suggested that a similar value should hold for field scale turbulence with significantly larger turbulent Reynolds numbers based on dimensional arguments.

A simple dimensional argument would suggest that the flux Richardson number should be equal to the gradient Richardson number, $R i_{g}=-N^{2}\left(\frac{\partial u}{\partial z}\right)^{-2}$, under the 
assumption that vertical eddy diffusivities for mass and momentum are equal. The gradient Richardson number represents the ratio of time scales associated with the stratification and the shear. Linear stability analyses (Miles, 1961; Howard, 1961) have defined a critical value of $R i_{g}=\frac{1}{4}$ as the theoretical threshold for growth of instabilities. This critical value has subsequently been interpreted as a threshold for the onset of turbulence, based in part on experimental evidence from laboratory (e.g., Thorpe, 1973), and field studies (e.g., Geyer and Smith, 1987). Instabilities can grow, triggering turbulent motions, for any flows with a gradient Richardson number less than the critical value. An equivalent value of the flux Richardson number would fall within the typical range, as reported by Osborn (1980), Ivey and Imberger (1991), Linden (1979) and others.

Recent studies have suggested values of the flux Richardson number outside of the 0.15 to 0.2 range. Barry et al. (2001) report the results of laboratory studies of gridgenerated turbulence, indicating that the mixing efficiency may decrease to zero at high values of $\frac{\varepsilon}{v N^{2}}$, particularly for $\frac{\varepsilon}{v N^{2}}>1000$, where it is speculated that the turbulent cascade cannot support the transfer of energy to scales small enough to support molecular diffusion and mixing. Field observations of the interior of two stratified lakes, where turbulence is driven mainly by internal wave breaking, with little mean shear, found negligible mixing efficiencies at $\frac{\varepsilon}{v N^{2}}$ values ranging from $10^{-1}$ to $10^{3}$ (Etemad-Shahidi and Imberger, 2001). These observations were based on the mean value derived from 
many instantaneous microstructure profiles. Although instantaneous buoyancy fluxes were observed, an equilibrium between upgradient and downgradient fluxes resulted in a negligible mixing efficiency. Similar upgradient fluxes have also been observed in the oceanic thermocline (Moum, 1996), suggesting dynamics similar to the lake interior. Etemad-Shahidi and Imberger suggest that the differences between these results and the earlier laboratory studies are due to differences in the mechanisms triggering the turbulence: oscillating grids and shear mechanisms in the laboratory experiments, and internal wave breaking in the lake interior and oceanic thermocline. Similarly, Balmforth et al. (1998) have suggested that grid-generated turbulence may not be representative of shear-produced turbulence.

Significant net buoyancy flux has been observed in the field associated with the turbulence generated by shear production at a highly energetic tidal front (Gargett and Moum, 1995). Estimates of the mean flux Richardson number for this flow were on the order of 0.4 . This brief review of mixing efficiencies suggests that there is considerable variability among existing observations, which may or may not be related to the turbulent generation mechanisms, as suggested by Etemad-Shahidi and Imberger (2001). In 1991, Imberger and Ivey recognized that a "strong debate" had been underway for several years regarding the magnitude of the flux Richardson number. Despite significant effort over the last decade, this debate remains unresolved, particularly for highly energetic flows.

\subsubsection{Entrainment Velocity}

One factor complicating the study of turbulence in energetic shear-stratified flows is the concept of entrainment. Morton et al. (1956) and Ellison and Turner (1959) define 
entrainment as the rate at which ambient fluid is added to an advancing plume.

Assuming plume motion predominantly in the horizontal plane, entrainment can be represented by a vertical velocity across some surface defined by the plume. In the context of stratified flows (e.g., Dallimore et al, 2001), it is traditional to discuss entrainment with respect to isopycnals. However, entrainment can also be observed in unstratified conditions, such as entrainment into a turbulent jet (e.g., Tennekes and Lumley, pp. 127-132). In this case entrainment can be considered with respect to a mean streamline bounding the initial fluid contained within the jet, or some volumetric subset of the initial jet. Assuming the receiving waters of a stratified plume are of constant density, the bounding edge of the plume must lie along an isopycnal, and the entrainment velocity at the edge, as calculated by either method, should be equal. Within the interior of the plume, significant differences between the two definitions of entrainment would be observed depending on the orientation of the respective surfaces.

Due to vagueness associated with generic use of the term "entrainment velocity", this term will not be used here to refer to any specific velocity structure. Instead, two vertical velocities will be defined which represent mean transport across isohalines, and across surfaces defined by the volumetric expansion of the original plume or river discharge. The first of these velocities, $\vec{w}_{h}$, can be defined as

$$
\vec{w}_{h}=\vec{u} \cdot \vec{n}_{h}
$$

where $\vec{w}_{h}$ can be referred to as a diahaline velocity, $\vec{u}$ is the local velocity vector, and $\vec{n}_{h}$ is the unit vector normal to the isohaline. The definition of the second velocity, which will be referred to as the jet entrainment velocity, by analogy to entrainment into and 
within an unstratified jet is similar, but taken with respect to surfaces bounding specific fractions of the initial discharge

$$
\vec{w}_{j}=\vec{u} \cdot \vec{n}_{j}
$$

where the subscript $\mathrm{j}$ refers to a volume defined surface. Each of these definitions provides an established reference frame and each defines a specific type of entrainment. The diahaline velocity represents entrainment into a mixed layer, which must incorporate fluid from two directions, if the layer is to grow, so that the diahaline velocity can be of either sign. The jet entrainment velocity represents entrainment into a region defined by a specified fraction of the original discharging fluid. In the case of a stratified plume discharged at the surface this implies that all jet entrainment velocities must be of the same sign, and directed upward. In either case, the entrainment velocity represents the vector difference between a mean flow streamline and a vector representing the isohaline or volume-defined surface. The simple diagrams in Figure 3.1 show the distinction between these two concepts of entrainment.

Ellison and Turner (1959) examined the entrainment of ambient fluid into a turbulent gravity current, relating the observed diahaline velocity across the bounding surface of the plume $\left(w_{\text {plume }}=w_{h(\text { bounding })}=w_{j(\text { bounding })}\right)$ to the mean horizontal flow, $U$. The constant of proportionality in their relationship, $E$, is referred to as the entrainment coefficient.

$$
E=\frac{w_{\text {plume }}}{U}
$$




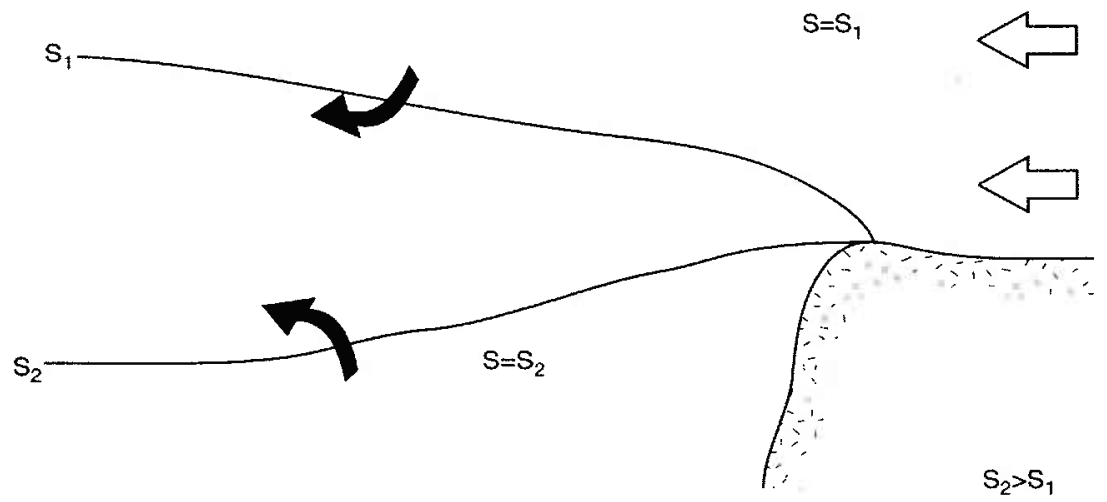

(a) Diahaline entrainment

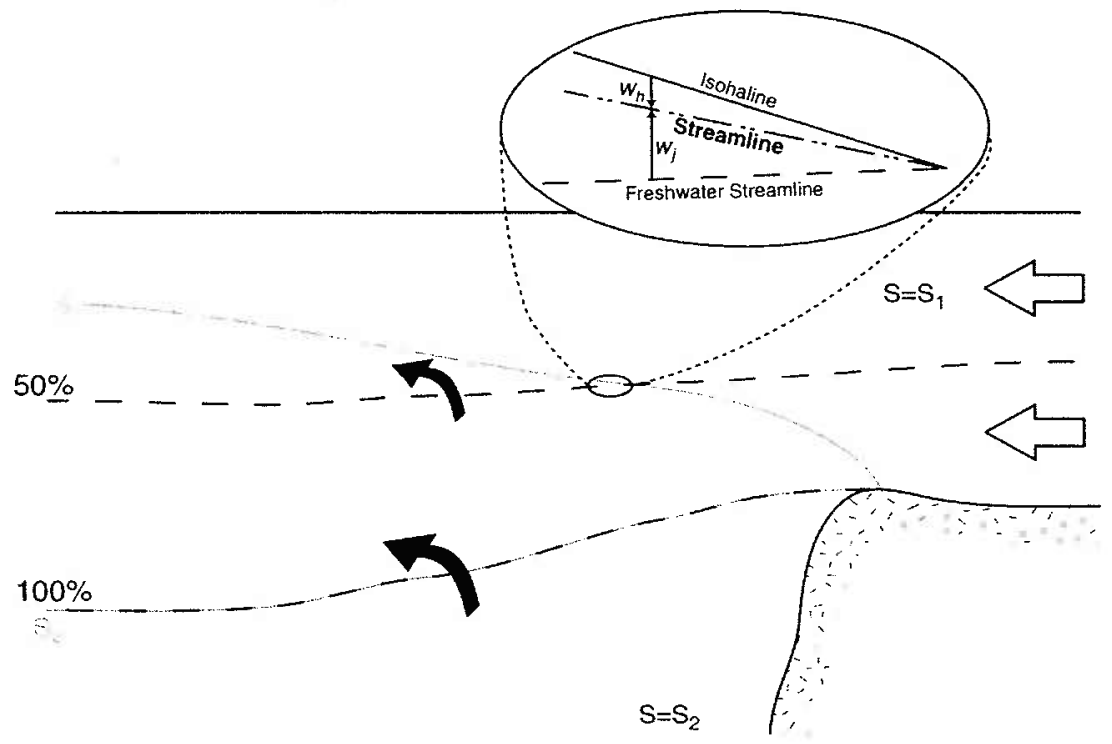

(b) Jet entrainment

Figure 3.1: Cartoons of entrainment relative to isohalines (a) and surfaces of constant initial discharge (b), where the dashed lines represent the bounding limits of the upper $50 \%$ and $100 \%$ of the initial discharge as it expands into the ambient fluid. In the first case, entrainment, as shown by the solid arrows can be of opposite sign at different levels in the water column, resulting in mixed layer growth. In the second case, the sign of the entrainment is always positive. The inset in (b) demonstrates that the entrainment velocity represents the vector difference between a mean streamline and a vector representation of the respective surface. 
The laboratory experiments carried out by Ellison and Turner, and more recent experiments by Pawlak and Armi (2000), suggest that the value of $E$ is a function of the bulk Richardson number, $\frac{g^{\prime} H}{U^{2}}$, where $H$ is the plume depth.

A unique aspect of the local diahaline velocity, $w_{h}$, is that all fluid crossing an isohaline must be diluted through mixing, in order to conserve salt on the isohaline surface. This balance (for the vertical direction only) can be represented, following McDougall (1984), as:

$$
\frac{\partial}{\partial z}\left(K_{z}^{\rho} \frac{\partial \rho}{\partial z}\right)=w_{h} \frac{\partial \rho}{\partial z}
$$

For a region of $\frac{\partial^{2} \rho}{\partial z^{2}}=0$, Equation (3.8) indicates that $w_{h}=\frac{\partial K_{z}^{\rho}}{\partial z}$, showing that the diahaline velocity is a direct consequence of the gradient in turbulence intensity, and is not an independent variable of the flow.

Vertical velocities induced in the water column by turbulence provide an efficient means for the vertical transport of salt and momentum in the water column, in addition to volume. Diahaline transport can also have important implications for hydraulic control as shown by Gerdes et al. (2002). In order to adequately characterize turbulent vertical transport from observations of total vertical transport, the effect of this mean vertical transport must be considered. 


\subsubsection{Turbulent Length Scales}

The range of turbulent length scales in shear stratified flows is typically represented by several internal scales, notably the Ozmidov scale, $L_{O}$, Corrsin scale, $L_{C}$, and Kolmogorov scale, $L_{K}$ : :

$$
\begin{aligned}
& L_{O}=\left(\frac{\varepsilon}{N^{3}}\right)^{\frac{1}{2}} \\
& L_{C}=\left(\frac{\varepsilon}{(\partial u / \partial z)^{3}}\right)^{\frac{1}{2}} \\
& L_{K}=\left(\frac{v^{3}}{\varepsilon}\right)^{\frac{1}{4}}
\end{aligned}
$$

The Ozmidov and Corrsin scales represent upper limits of eddy size with respect to deformation by stratification and shear, respectively, as described by Smyth and Moum (2000). The Kolmogorov scale represents the range of smaller scales where TKE is dissipated (Tennekes and Lumley, 1972, p. 67). Each of these scales is a function of the dissipation, $e$, implying that an a priori knowledge of the turbulence field is necessary for their estimation.

An overturn scale, $L_{T}$, is a much more readily accessible quantity from observational data, and is often assumed equal to the Ozmidov scale, although the actual relationship between these two length scales may be much more complicated (Baumert and Peters, 2000). The overturn scale is defined as the rms displacement distance identified when comparing an observed vertical density profile to its stably re-sorted counterpart (Thorpe, 1977). Assuming a suitable prediction of $L_{O}$ can be derived from $L_{T}$ (e.g. Osborn, 1980; Dillon, 1982; Baumert and Peters, 2000) dissipation can then be estimated using (3.9). 
The overturn scale, however, remains a statistical approximation of an outer turbulence scale based on observations.

As pointed out by Imberger and Ivey (1991), only two independent variables exist in a pure shear-stratified flow: one representing the shear, $\Delta u$, and the other representing the stratification, $\Delta \rho$, which can be represented as a reduced gravity, $g^{\prime}=g \frac{\Delta \rho}{\rho_{o}}$. Furthermore, Imberger and Ivey suggest length and dissipation scales from these variables based on simple dimensional analysis. Representative dissipation and mixed layer length scales for the flow should be proportional to these stratified shear scales:

$$
\begin{aligned}
& \varepsilon=\gamma\left(\Delta u g^{\prime}\right) \\
& \delta=\varphi\left[\frac{(\Delta u)^{2}}{g^{\prime}}\right]
\end{aligned}
$$

where $\delta$ represents the thickness of the mixed layer, and $\gamma$ and $\varphi$ are coefficients of proportionality, which may vary as the shear layer develops.

In this chapter, the turbulence associated with the highly stratified front at the mouth of the Fraser River is investigated. The goals of the study were to develop an effective method for estimating turbulent quantities from bulk water column observations, to relate the observed turbulent quantities to mean scales representative of the shear-stratified flow, and to comment on the observed mixing efficiencies in the high Reynolds number turbulence near the front. At the Fraser River front, both of the shear-stratified independent variables, $\Delta u$, and $g^{\prime}$, are high compared to other oceanic and coastal 
flows, providing an ideal location to observe energetic turbulence with high values of

$$
\frac{\varepsilon}{v N^{2}}
$$

\subsection{The Fraser River and the 1999 Field Effort}

The study was conducted at the mouth of the Fraser River, in southwestern British Columbia, Canada, as introduced in Chapter 1. Bathymetric and hydrographic considerations relevant to the local region at the mouth have been discussed in Chapter 2 . As discussed previously, the dynamics in the estuary are dominated by an interaction between tidal and discharge energies. Tides in the Strait of Georgia are mixed semidiurnal and diurnal, with amplitudes typically ranging from 2.5 to 4 meters. River discharge is dominated by the summer freshet, during which discharge typically increases by a factor of ten relative to low flow, when snow pack from the higher elevations of the inland mountains melts. The highly energetic estuarine environment that results from the interaction of these two strong forcing mechanisms is characterized by intense stratification and intense horizontal salinity gradients. The resulting salt wedge exhibits salinity differences of greater than $20 \mathrm{psu}$ across only a few meters in the vertical and less than 500 meters in the horizontal at the head. This salt wedge advances landward some 10 to $20 \mathrm{~km}$ into the channel on each tidal cycle, only to be flushed back to the mouth daily during the strong ebb (Geyer and Farmer, 1989). At this point in the cycle, after the salt wedge has retreated to the mouth, there is a period of quasi steady-state dynamics as the front location remains stable for several hours, prior to beginning its next advance 
into the channel on the flooding tide. The frontal region during this period will be referred to as the "lift-off" zone, as in Chapter 2, and is the focus of the analyses presented in the remainder of this chapter.

The data evaluated in this study was collected on July 25,1999 , five days past neap tide, during a summer characterized by an unusually large, and delayed, freshet discharge. River discharge on July 25 th was approximately $7,500 \mathrm{~m}^{3} \mathrm{~s}^{-1}$, as measured at the most seaward gauging station, located at Hope, approximately $150 \mathrm{~km}$ upstream of the mouth. This compares with an average peak freshet discharge of approximately $7,000 \mathrm{~m}^{3} \mathrm{~s}^{-1}$ typically occurring in early June, and a 1999 peak of approximately 11,000 $\mathrm{m}^{3} \mathrm{~s}^{-1}$ which occurred in late June.

Data was collected from shipboard instrumentation aboard the R/V Clifford Barnes (University of Washington), including two hull-mounted Acoustic Doppler Current Profilers (ADCPs), operating at $1200 \mathrm{kHz}$ and $300 \mathrm{kHz}$, and a tow-yoed Ocean Sensors 200 Series CTD unit. Combined use of the two ADCPs allowed for consistent bottom tracking coupled with a vertical resolution on the order of $25 \mathrm{~cm}$ across the top $25 \mathrm{~m}$ of the water column. Vertical resolution of the CTD unit was on the order of 5 to $10 \mathrm{~cm}$. The data discussed herein was collected from repeated passes along a transect line across the front, oriented parallel to the discharging river flow as shown on Figure 3.2. Measurements were taken from the vessel as it drifted seaward with the flow across a typical length of 2 to $3 \mathrm{~km}$. Three passes were completed within approximately two hours, representing the essentially steady state conditions during the low tide period. 


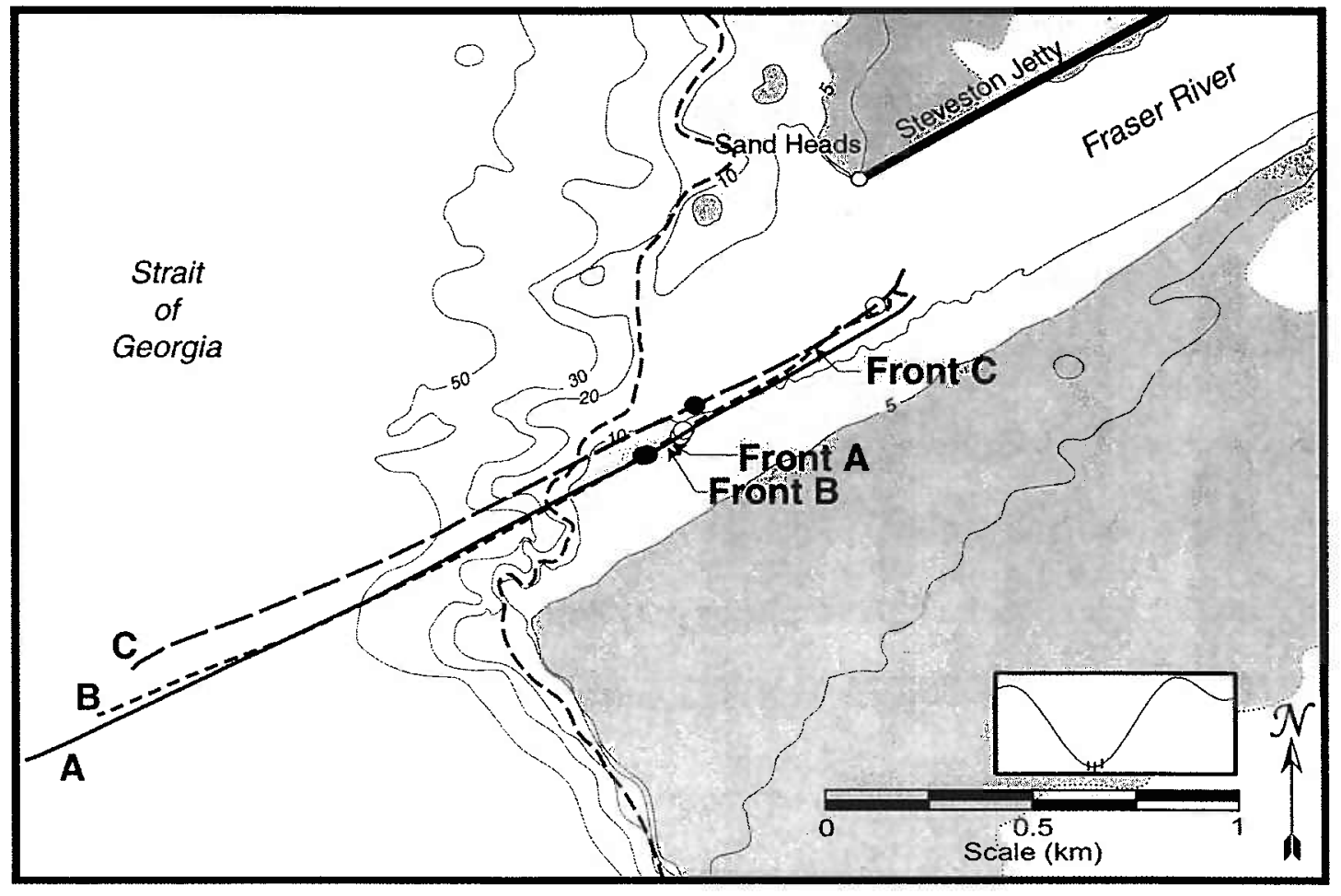

Figure 3.2: Plan view of estuary mouth, showing ship tracks of three passes during low tide on July 25,1999 . Dashed line represents the $12 \mathrm{~m}$ isobath, approximating the location of the bathymetric break. Observed location of the front during each pass is indicated by the open and closed circles, representative of the absence of 14 psu salinity fluid, and the presence of 14 psu salinity fluid, respectively. 


\subsection{Hydrography Seaward of the Front}

Cross sections through the front are shown in Figure 3.3, representing all three passes. The strong stratification, as indicated by the close spacing of the isohalines, and the convective acceleration of the discharge as it passes the front, can be clearly seen in the three panels. The bold lines in the figure are mean fresh water streamlines, and represent the bounding surfaces for specific fractions of the original river discharge. These surfaces were estimated from the measured salinity and velocity fields based on fresh water conservation, as follows. Defining the salinity of pure Georgia Strait water as $S_{o}=27 \mathrm{psu}$, a fresh water flux conservation equation can be written as:

$$
Q_{o}=\int_{z_{S_{o}}}^{0} u \frac{\left(S_{o}-S\right)}{S_{o}} b d z
$$

where $Q_{o}$ is constant and equal to the total fresh water flux in the cross section, $u$ is the velocity parallel to a mean upper layer flow direction (defined as the $x$ direction), $S$ is the

local salinity, and $b=b(x, z)$ a relative plume width in the cross-stream direction. Using (3.11) and some simple assumptions regarding the shape of the vertical profile of $b$, the value of $b$ can be determined at each point in the cross section, allowing the trajectory of fresh water streamlines to be generated following a specified fraction of the total fresh water flux. The fresh water streamlines shown in Figure 3.3 represent $20^{\text {th }}$ percentiles of the total freshwater flux. Overall, five different assumptions regarding the shape of the width profile relative to depth were compared to evaluate the sensitivity of the process to the uncertainty in the shape of the plume. These included a depth-constant width, and several variations of linear and parabolic width distributions. In these cases, the plume is 
(a)

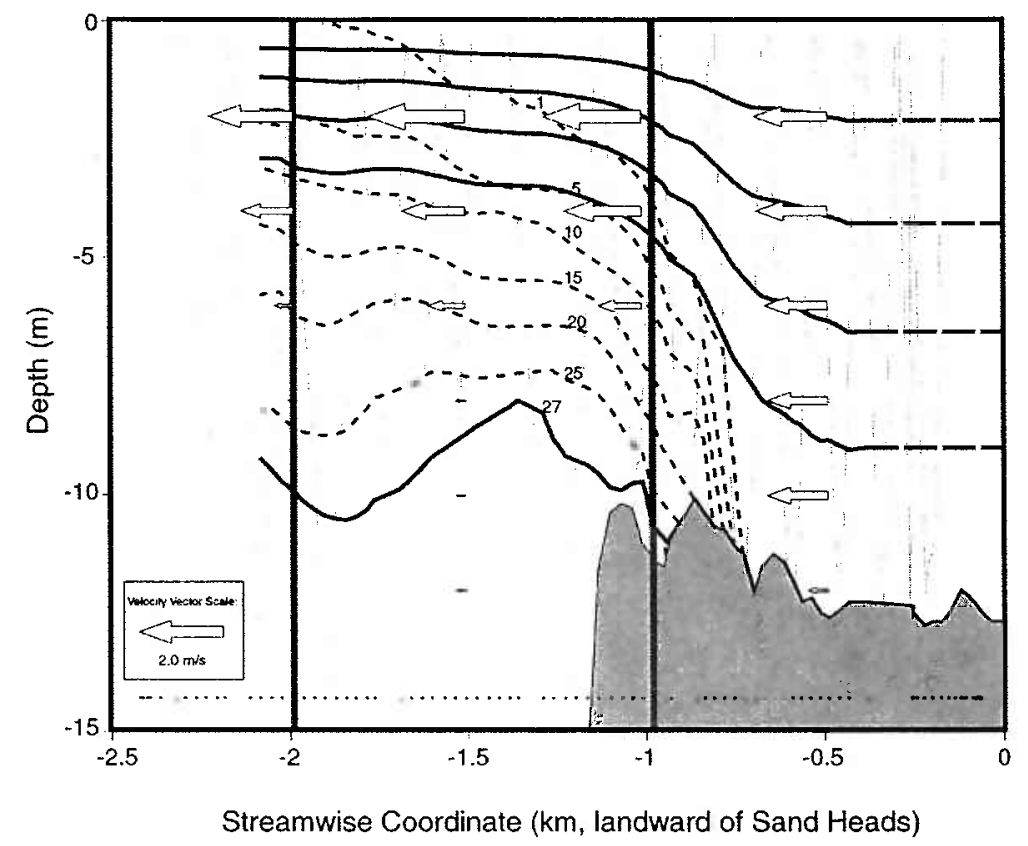

(b)

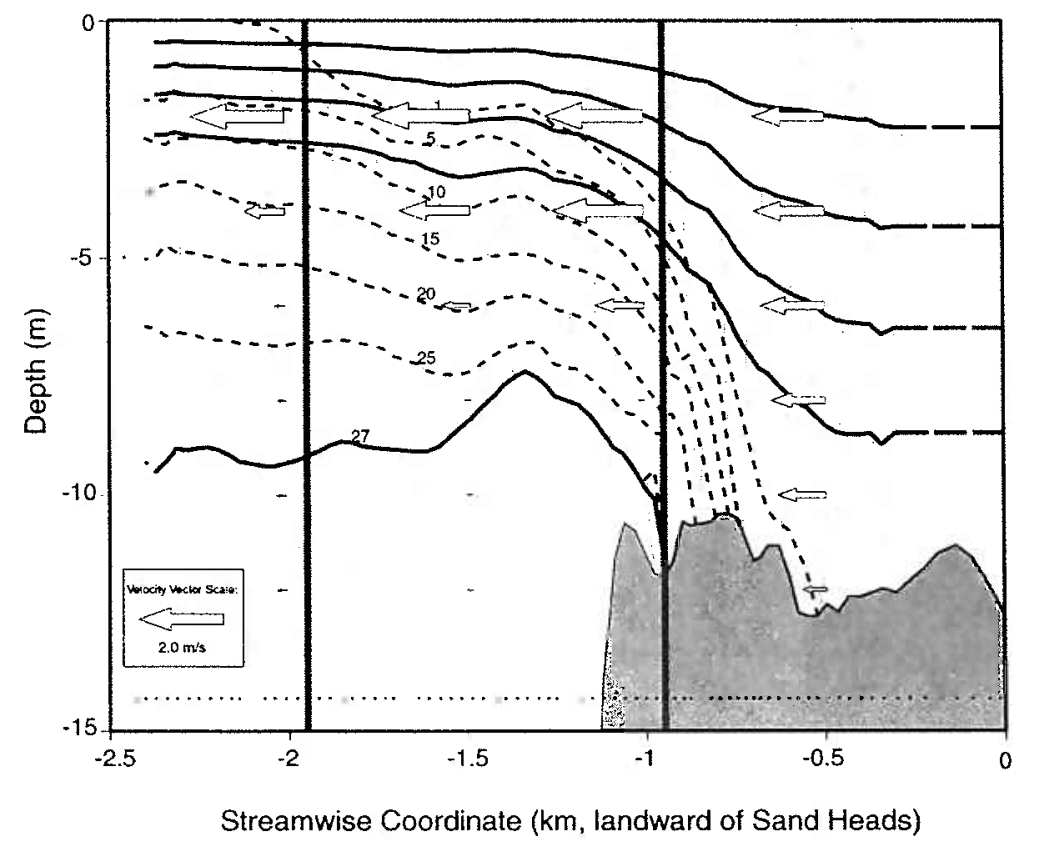

Figure 3.3: Cross section through the front for the three passes, (a) A, (b) B, and (c) C. Dashed contours represent salinity, in psu. Arrows represent streamwise velocity, based on the scale in the lower left hand corner of the figure. Bold solid lines are freshwater streamlines, calculated through the conservation of freshwater as described in the text. CTD tow-yo track is shown in the background of each figure, and ADCP profile locations are identified by the dots near the bottom of each panel. Regions used for control volume analyses are delineated by the two vertical lines. 


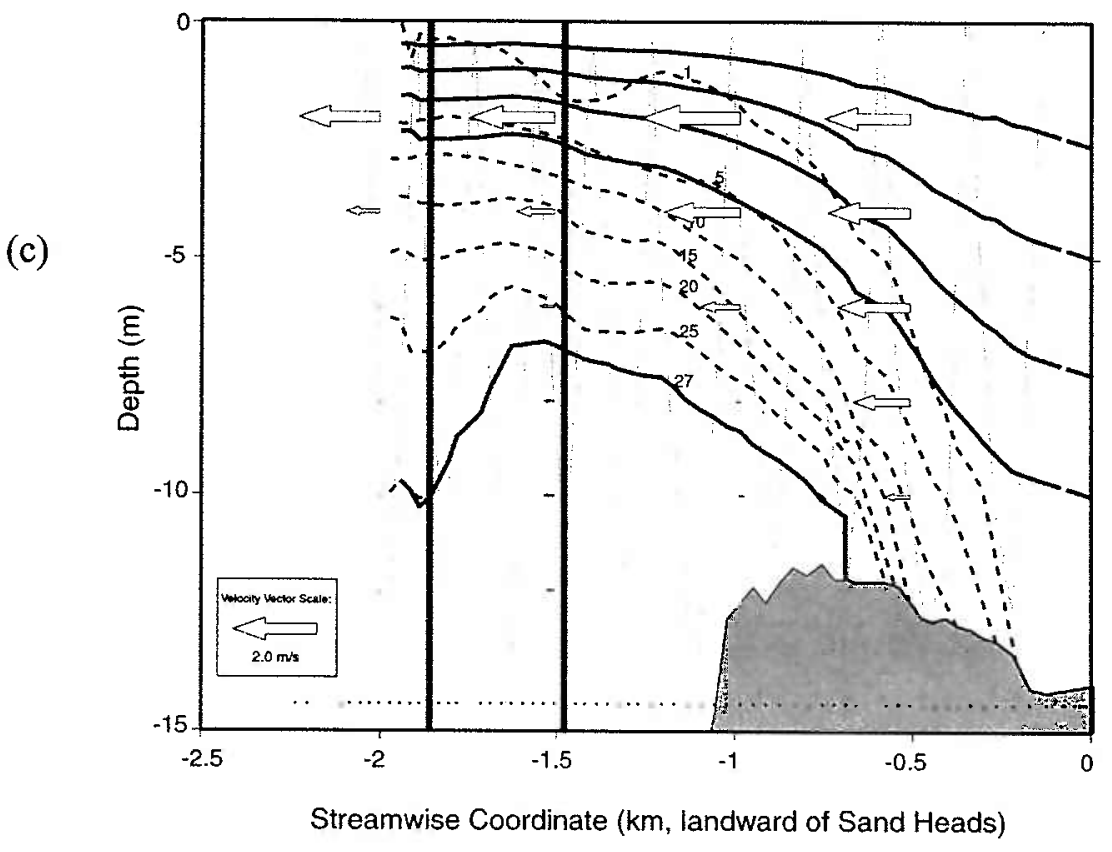

Figure 3.3 (c): For caption see previous page. 
assumed to be wider near the surface in order to approximate an increased spreading near the surface due to buoyancy.

The angle of the freshwater streamlines relative to the isohalines in Figure 3.3 implies that significant cross streamline entrainment (i.e., "jet entrainment") is occurring at all depths seaward of the front, likely coupled with a high degree of turbulent mixing, and the generation of mean diahaline velocities. Analytical and numerical models of river plumes (Garvine, 1982), and arrested fronts (Farmer and Armi, 1986; Armi and Farmer, 1986) typically assume that the Froude number within the plume increases monotonically seaward of the front, implying invisicid conditions within the lift-off zone. The Froude number was introduced in Chapter 1 and discussed with respect to hydraulic control in Chapter 2. Here the upper layer Froude number is most significant, given that the lower layer is arrested:

$$
G^{2} \approx F_{1}^{2}=\frac{u_{1}^{2}}{g^{\prime} h_{1}}
$$

where the subscript 1 refers to representative upper layer values. In Chapter 2 it was shown that for fronts oriented obliquely to an oncoming flow, the Froude number need not maintain a critical value of unity, as suggested in much of the literature (e.g., Farmer and Armi, 1986), but that a Froude angle, defined as $\phi=\sin ^{-1}\left(G^{-1}\right)$ is critical when it is equal to the angle between the front and the oncoming flow. In the present context, it is the change in Froude number in the streamwise direction, and not the local value of the Froude number, that is of primary concern. 
A plot of the upper layer Froude numbers associated with each of the three passes is presented in Figure 3.4. Here we see supercritical values of the Froude number at the front, and an increase in Froude number for approximately the first $700 \mathrm{~m}$ seaward of the front. Beyond about $700 \mathrm{~m}$, however, the profiles are characterized by a steady decrease. This may indicate a loss of energy through dissipative mixing processes, suggesting that the inviscid assumption may not adequately characterize conditions in the lift-off zone.

Both the orientation of the fresh water streamlines, and the decreasing trend in the Froude number profiles indicate that significant mixing may be occurring near the front. The following section outlines a procedure used to estimate TKE production and buoyancy flux in the lift-off zone using the available ADCP and CTD data.

\subsection{Estimation of Turbulent Fluxes}

The control volume analysis used for the estimation of turbulent transport quantities relies on the three conservation constraints of volume, salt, and momentum. The three unknowns resolved are the mean velocity across the bottom surface of the control volume (assuming the top surface of the control volume is taken coincident with the surface), and the vertical turbulent transport of both salt and momentum. The results of the turbulent flux calculations for salt and momentum are independent of the shape of the control volume, but the mean velocity resulting from the analyses is relative to the shape of the bottom control volume surface. For this reason, it is most instructive to use bottom control volume surfaces aligned with isohaline and/or freshwater streamline surfaces. 


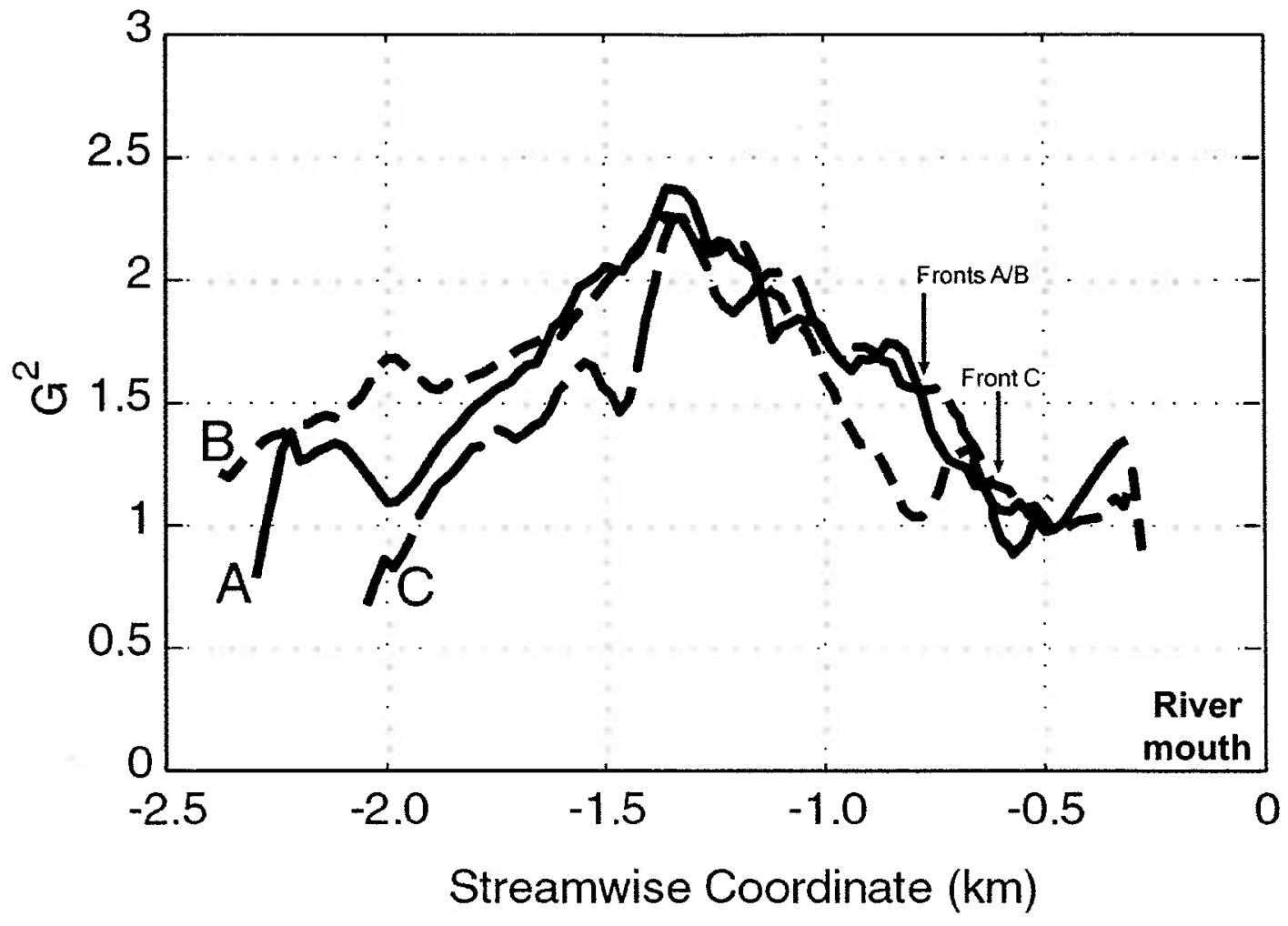

Figure 3.4: Profiles of the squared composite Froude number, G, along each of the three ship-track passes presented in Figure 3.2. The observed front locations are noted for each pass. 
Volume conservation is used to identify the mean velocity across the bottom control volume surface. Written as an integral across the control volume surface, this can be expressed as:

$$
\iint_{C S} \vec{U} \cdot d \vec{A}=\frac{\partial V}{\partial t}
$$

where $\vec{U}$ represents the local velocity at the control volume surface, $\vec{A}$ is a unit vector perpendicular to the surface, the integral represents summation over the entire control volume surface, and $V$ is the total volume contained within the control surface. Assuming no contribution from lateral fluxes, the control volume equation can be rewritten in differential form as:

$$
\frac{\partial}{\partial x}\left[\int b u d z\right]+\bar{w} b=\frac{\partial}{\partial t}\left[\iint b d z d x\right]
$$

where the integrals are taken across the vertical limits of the control volume, and $\bar{w}$ represents the mean vertical velocity, which is equal to $w_{h}$ if the bottom control volume surface is coincident with an isohaline, or $w_{j}$ if the bottom of the control volume is aligned with a freshwater streamline.

A similar treatment of the salt balance allows for estimation of the total vertical salt flux, $\overline{S w}$, where

$$
\iint_{C S} S \vec{U} \cdot d \vec{A}=\frac{\partial}{\partial t}\left[\iint_{C V} S d V\right]
$$

represents the control surface equation, with salinity represented by $S$. This can be written in differential form as: 


$$
\frac{\partial}{\partial x}\left[\int S b u d z\right]-\overline{S w} b=-\frac{\partial}{\partial t}\left[\iint S b d z d x\right]
$$

Once an estimate of the total vertical salt flux has been generated, the turbulent part can be extracted following a Reynolds decomposition, and making use of the vertical velocity estimated in (3.14):

$$
\overline{S^{\prime} w^{\prime}}=\overline{S w}-\bar{S} \bar{w}
$$

where $\bar{S}$ is the mean salinity of the bottom control volume surface. If the bottom surface is coincident with an isohaline, $\bar{S}$ is equal to the salinity associated with the isohaline.

Treatment of the momentum balance requires knowledge of the net force on the control volume associated with the local pressure, but is otherwise similar to the salt balance. Estimation of the turbulent momentum flux can be represented by the following equations:

$$
\begin{gathered}
\sum F_{x}=\iint_{C S} u \rho \vec{U} \cdot d \vec{A}+\frac{\partial}{\partial t}\left[\iiint_{C V} u \rho d V\right] \\
\frac{\partial}{\partial x}\left[\int S b u d z\right]-\overline{S w} b=-\frac{\partial}{\partial t}\left[\iint S b d z d x\right] \\
\overline{u^{\prime} w^{\prime}}=\overline{u w}-\bar{u} \bar{w}
\end{gathered}
$$

where $F_{x}$ represents force in the streamwise direction associated with the local pressure.

The size of the control volumes used for the analyses was on the order of 0.5 to $1 \mathrm{~km}$ in the $x$ direction (as identified on Figure 3.3). The top surface of the control volume was always coincident with the fluid surface, and lateral width was defined by $b$ as in (3.11). The bottom control volume surfaces were represented by isohalines for the diahaline 
velocity and turbulent salt flux estimates. Due to the wide spacing of isohalines near the surface, where a majority of the momentum flux occurs, an independent set of calculations for the momentum flux was conducted using bottom control volume surfaces coincident with the fresh water streamlines as identified on Figure 3.3.

A schematic of the control volume process is shown in Figure 3.5. The implementation of each step of this calculation process is discussed below in further detail. The time dependent terms incorporated into Equations (3.13) through (3.20) were estimated from the data by comparing subsequent passes, but were generally small due to the quasi steady state nature of the plume during the sampling transects.

\subsubsection{Measured Quantities}

Mean velocity and salinity profiles for the three passes are presented in Figure 3.6. These profiles identify the two independent variables in the shear stratified environment, $\Delta u$, and $\Delta \rho$. The highly sheared streamwise velocity profiles are presented in the first panel, characterized by a $\Delta u$ on the order of $2 \mathrm{~m} \cdot \mathrm{s}^{-1}$. Likewise, the salinity profiles identify a zone of strong stratification between roughly 3 and 8 meters below the surface, characterized by a $\Delta S$ on the order of 20 psu, or an equivalent $g$ ' value of approximately $0.15 \mathrm{~m} \cdot \mathrm{s}^{-2}$

Profiles of the gradient Richardson number, based on the mean profiles of Figure 3.6, are shown in Figure 3.7. Each of the three profiles contains a significant portion of the water column with $R i_{g}$ values below the critical value of $1 / 4$. These critical regions, shown shaded in Figure 3.7, are generally positioned between 2 and $6 \mathrm{~m}$ below the surface. It is at these depths that the most significant mixing is expected. 


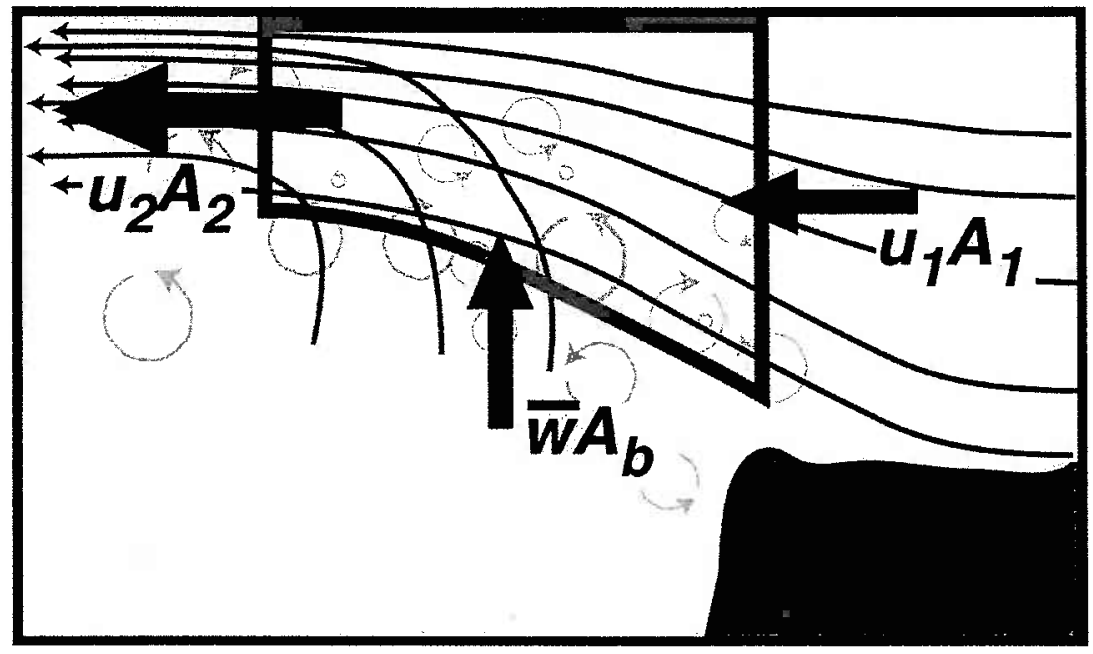

(a) Volume Conservation

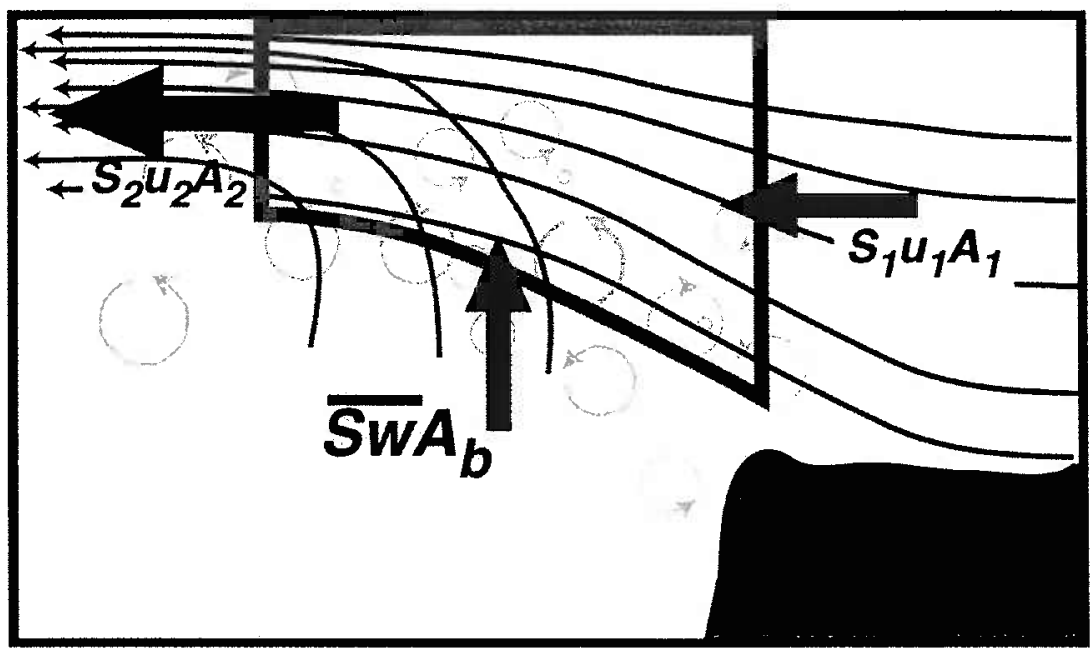

(b) Salt Conservation

Figure 3.5: Two-dimensional schematic of the control volume method, identifying the volume conservation process (a) for isolating the mean vertical entrainment velocity $\bar{w}$, and the salt conservation process (b), which allows for the calculation of the total vertical slat flux, $\overline{S w}$. In both cases, A represents the projected area of a specific face of the control volume, $S$ is salinity, and $u$ and $w$ are streamwise and vertical velocities, respectively. The calculation process shown in these schematics is simplified in that incoming and outgoing fluxes are not represented as integrals over the surface, and the time dependent terms are not represented. The control volume analysis for momentum is not shown, but is similar to the salt analysis, with the additional component of an integration of pressure forces across the control surface. 

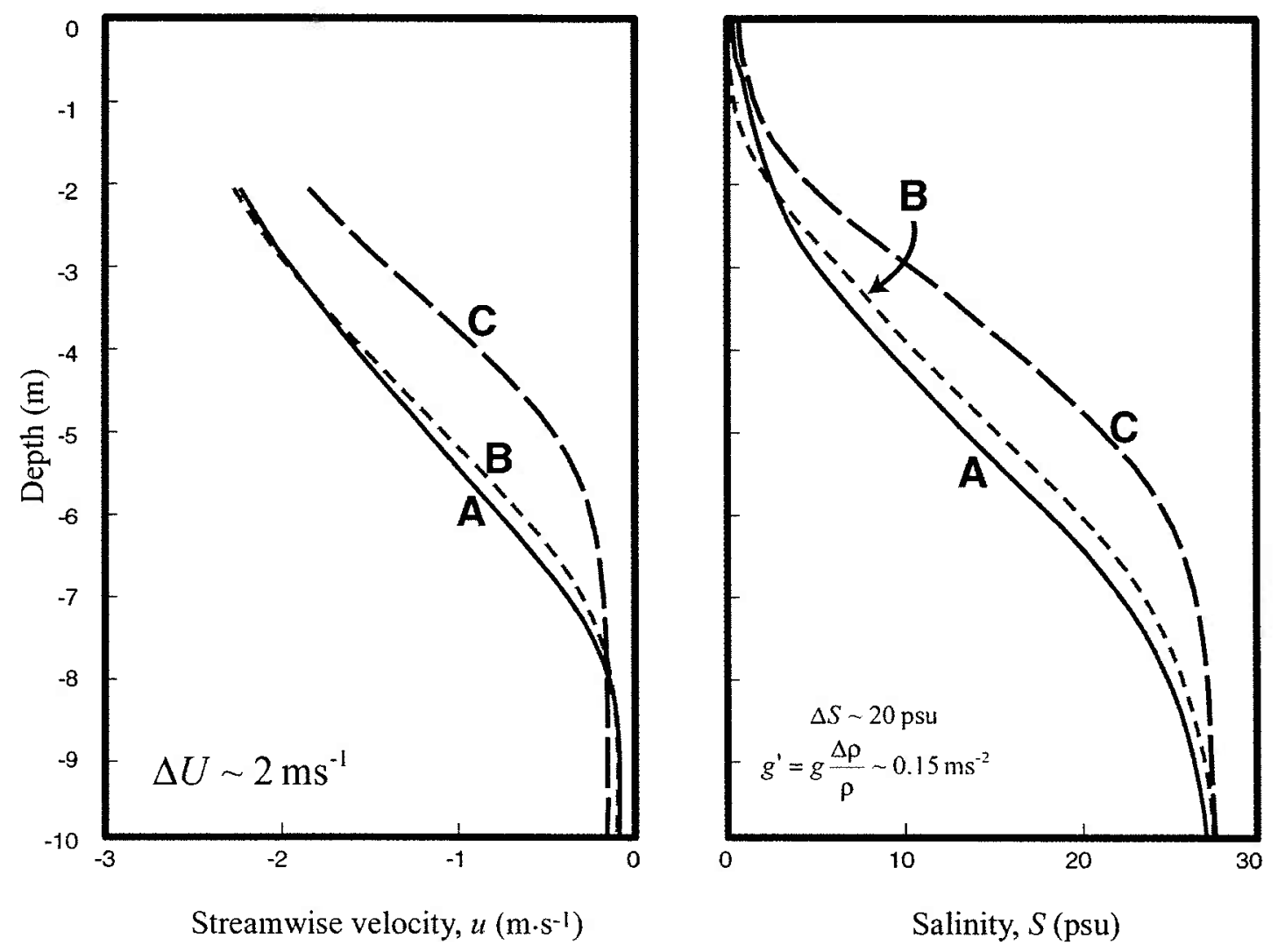

Figure 3.6: Vertical profiles of measured quantities, velocity and salinity, in the control volume region for each of the three lift-off passes. Plots represent mean profiles across the control volume region, which has a streamwise dimension of approximately $1 \mathrm{~km}$ for passes $\mathrm{A}$ and $\mathrm{B}$, and 


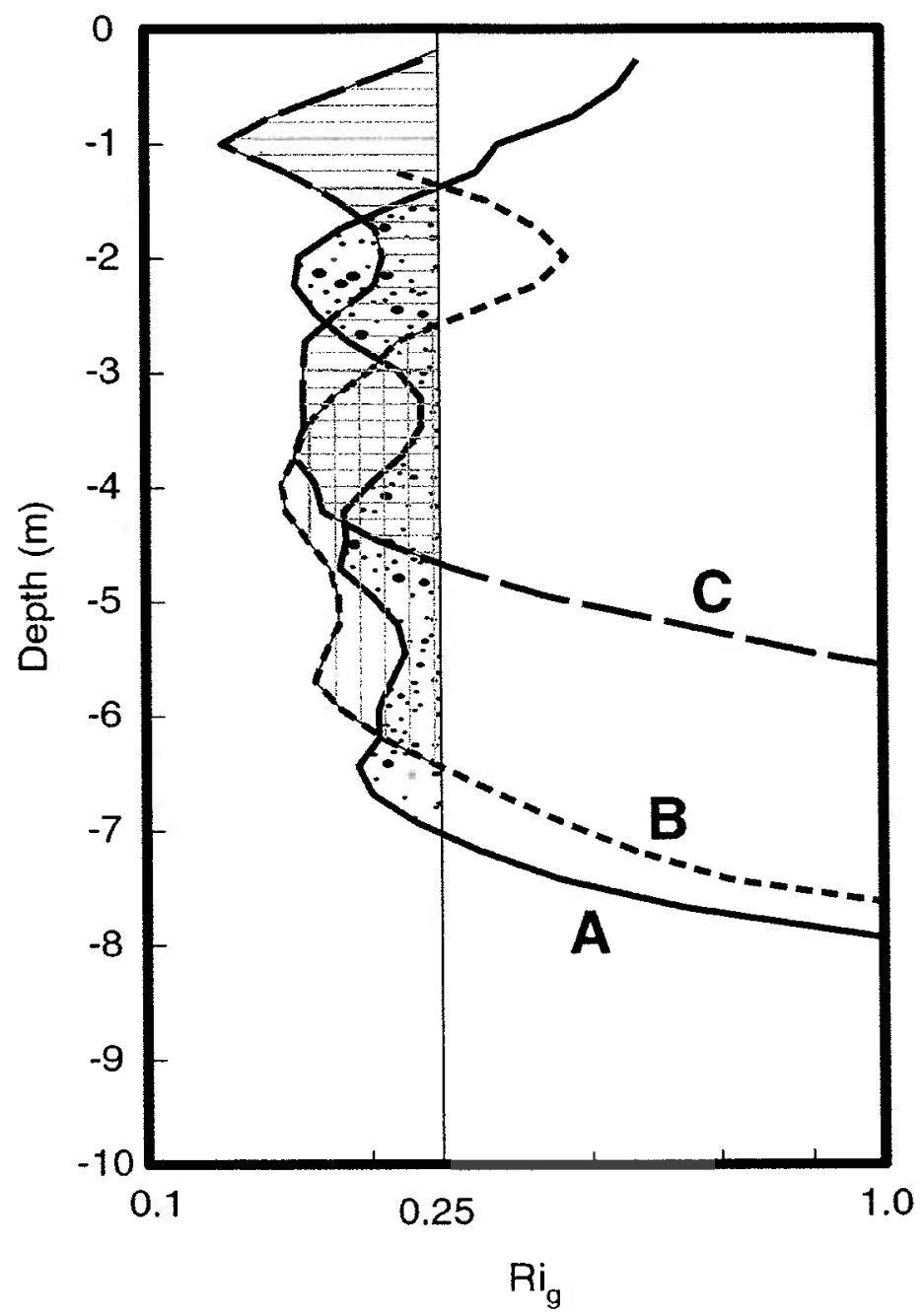

Figure 3.7: Vertical profiles of the gradient Richardson number, based on the mean quantities shown in Figure 3.6. Profiles are smoothed across approximately $1.5 \mathrm{~m}$ in the vertical. The vertical line represents a value of $1 / 4$, and the shaded regions indicate the locations where the gradient Richardson 


\subsubsection{Volume Conservation and Vertical Velocity}

The concept of entrainment velocity was discussed in Section 3.1, where two vertical velocities were defined relative to specific surfaces within the developing flow structure. In the context of the Fraser lift-off, these two surfaces are represented by the isohalines, and the freshwater streamlines. Under inviscid conditions, streamlines would follow lines of constant density, which in this case would be coincident with isohalines. The migration of isohalines across the freshwater streamlines is indicative of entrainment.

Mean vertical entrainment velocity in the Fraser lift-off is calculated from observations of volume entrainment within the seaward flowing discharge plume, following the calculation scheme outlined above. Initial calculations utilized bottom surfaces representing isohalines, in order to generate a vertical profile of the diapycnal velocity, $w_{h}$. The process was then repeated using bottom surfaces coincident with the fresh water streamlines associated with each percentile increase in flow fraction, providing a vertical profile of $w_{j}$.

Mean vertical profiles of $w_{h}$ and $w_{j}$ for each of the passes are presented in the two panels of Figure 3.8. Error ranges shown on Figure 3.8 represent the standard deviation of the results associated with the various width shape assumptions, as discussed above. This error is negligible at depths less than approximately five meters, and generally less than $30 \%$ at depth. The profiles of $w_{h}$, in the panel at the left show entrainment of both surface and deep water into a developing mixed layer centered at approximately 3 to $4 \mathrm{~m}$ depth and approximately 10 to 14 psu. In the panel on the right, the profiles of $w_{j}$ 

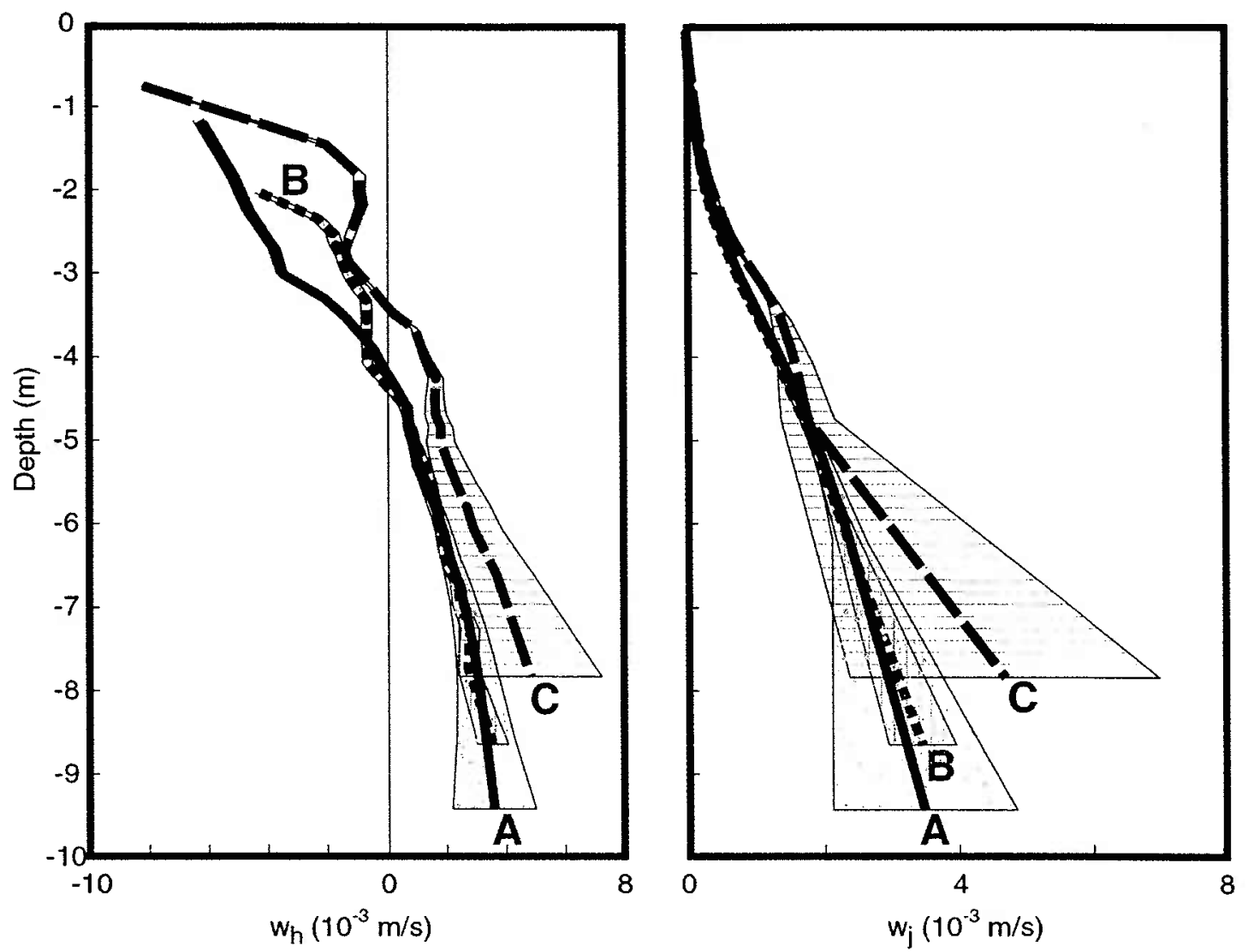

Figure 3.8: Vertical profiles of the mean vertical velocities, $w_{h}$ and $w_{j}$, as calculated from the control volume analyses. Estimates are bracketed by error bounds, based on the standard deviation of the results using five different width assumptions. 
indicate that ambient fluid is being entrained into all portions of the initial discharging plume, with entrainment increasing relative to depth. At the bounding limit of the plume, $w_{h}$ is equal to $w_{j}$ because the bottom boundary of the expanding discharge is coincident with the $27 \mathrm{psu}$ isohaline.

\subsubsection{Salt Conservation}

Total vertical salt transport is calculated as described above, utilizing a series of isohaline surfaces as the bottom control volume surface for purposes of generating a vertical profile. In this case, the vertical velocity represented by $w_{h}$ is used with the salinity of the isohaline surface to estimate the component of the total vertical salt flux attributable to entrainment.

The first panel of Figure 3.9 shows profiles of turbulent salt flux, expressed as buoyancy flux, as in (3.1):

$$
B=g \beta \overline{S^{\prime} w^{\prime}}
$$

where $\beta=\left(\frac{1}{\rho} \frac{\partial \rho}{\partial S}\right)=0.77 \times 10^{-3} \mathrm{psu}^{-1}$. In the second panel, profiles of eddy diffusivity, derived from the turbulent salt flux profiles using (3.3) are plotted. The buoyancy flux profiles are similar for the three passes, which peak near $2 \times 10^{-4} \mathrm{~m}^{2} \mathrm{~s}^{-3}$ in the midst of the pycnocline near $4 \mathrm{~m}$ depth. The profiles of eddy diffusivity indicate values of $K_{z}^{\rho}$ on the order of 2 to $6 \times 10^{-3} \mathrm{~m}^{2} \mathrm{~s}^{-1}$ across the middle portion of the water column.

All of the profiles in Figure 3.9 are shown bracketed by the standard deviation associated with the various width shape assumptions, as above. The negative values of both buoyancy flux and eddy diffusivity observed below about $8 \mathrm{~m}$ for pass B are 

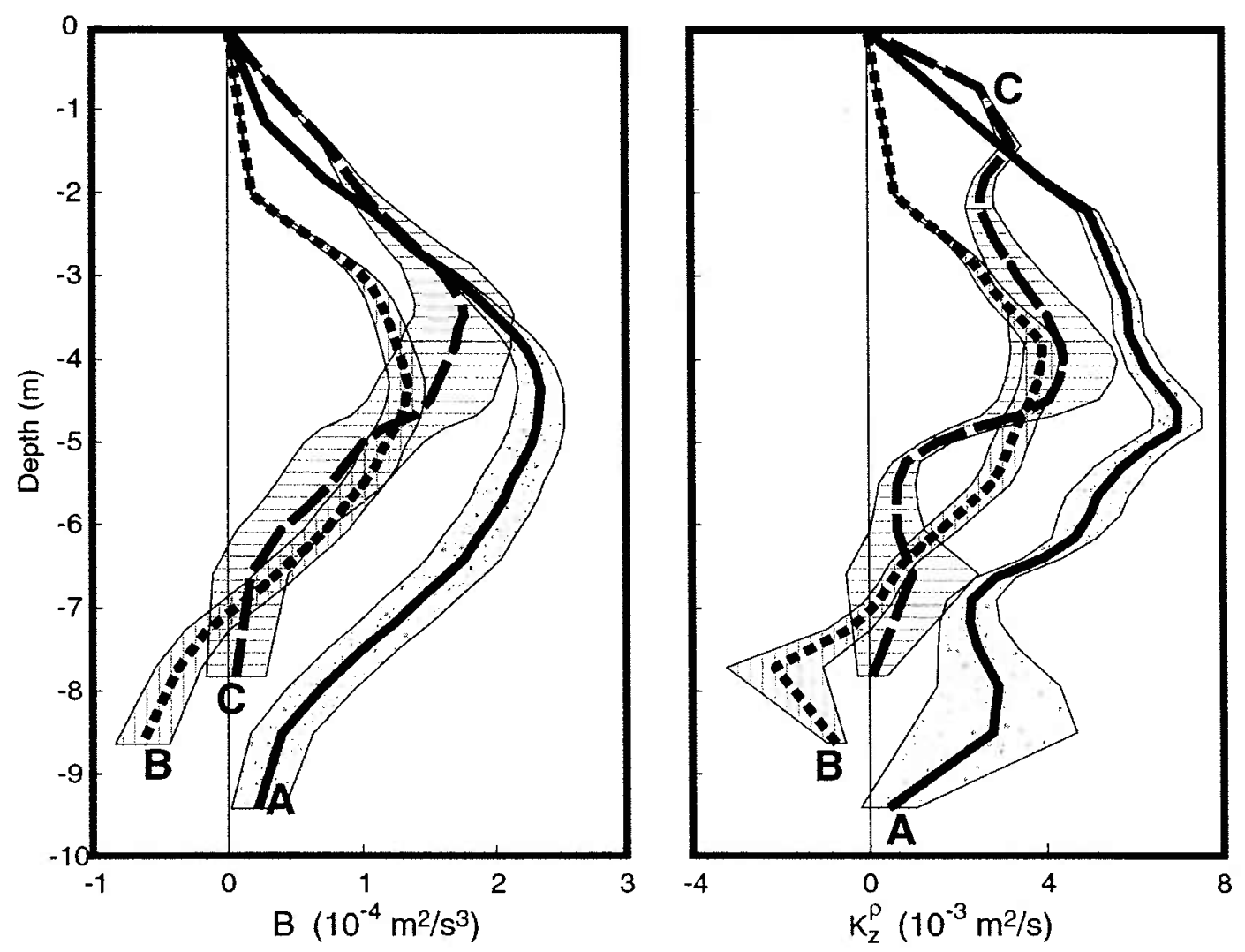

Figure 3.9: Buoyancy flux (B), and eddy diffusivity $\left(K_{z}^{\rho}\right)$ profiles from control volume analysis. Each estimate is bracketed by standard deviation as in Figure 3.8. Note that estimates below approximately 5 to 6 meters are also influenced by unresolved lateral fluxes as discussed in the text. 
unrealistic. They can be explained by unresolved lateral influxes of salt and volume, which are discussed more thoroughly later in this chapter.

\subsubsection{Momentum Conservation}

The procedure for calculation of the total vertical momentum flux is similar to the volume and salt calculations, but complicated by the necessity of estimating the net streamwise directed force on the control volume associated with the pressure gradient, as described above. A hydrostatic force balance is assumed, including both barotropic and baroclinic contributions. The baroclinic contribution is calculated based on density profiles within the control volume. The barotropic contribution is determined from a surface gradient, $\partial \eta / \partial x$, estimated as the gradient required to produce a layer of no motion, as observed at depths sufficiently below the plume. The reference level for this layer was typically taken at $10 \mathrm{~m}$ below the surface, in order to extend the control volumes landward of the bathymetric break. In the case of Pass $C$, which occurred at the very early stages of the flood, weak landward currents in the lower layer near the front produced conditions which invalidated the no motion and zero-stress-divergence assumptions, so the control volume region had to be shortened and moved offshore. The limits of the control volume regions used for each of the three passes are shown on the cross sections in Figure 3.3.

Profiles of the surface elevation, $\eta$, are shown in Figure 3.10, where the layer of no motion method is compared with two other estimates: one generated using a Bernoulli

approach along the extrapolated surface velocities (i.e., $\eta=\frac{1}{2 g} u^{2}+\frac{1}{g} \int \frac{\partial u}{\partial t} d x$ ), and the 

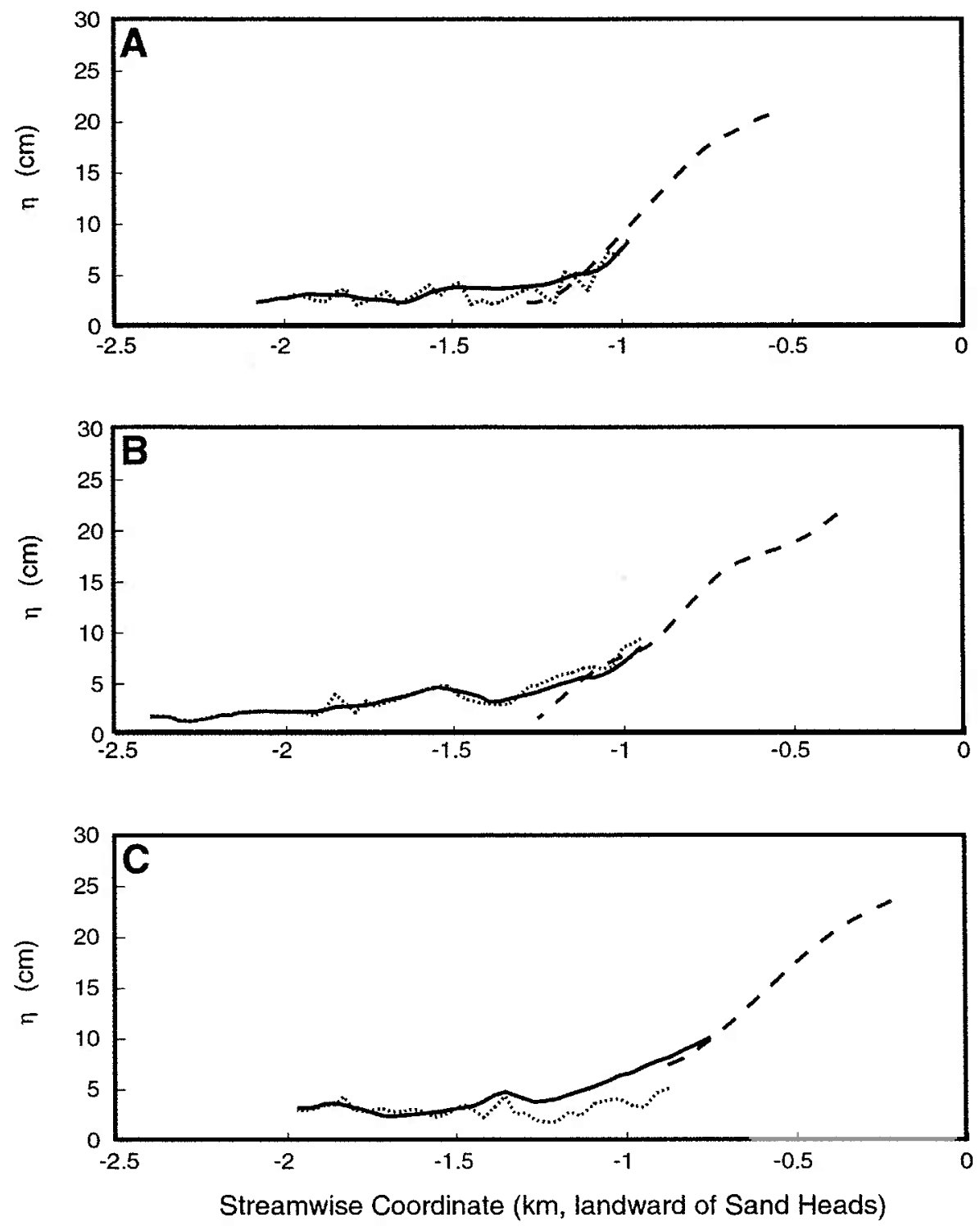

Figure 3.10: Estimates of surface elevation across the lift-off region based on three different methods of estimating the surface slope, $\partial \eta / \partial x$. The solid line represents the assumption of a layer of no motion, and zero stress divergence at a depth of $10 \mathrm{~m}$ below the surface. These results were used for subsequent momentum budget calculations. The dotted line represents the surface profile required to force the stress to zero at depth $(\sim 15 \mathrm{~m}$ below the surface) using a modified form of the control volume analysis. The dashed profile was derived using a Bernoulli approach based on the extrapolated surface velocities, assuming zero stress divergence at the surface. The dashed profile is terminated in the seaward direction where surface salinities exceeded 1 psu, providing evidence of mixing and an invalidation of the zero stress divergence assumption. 
other determined as the surface elevation profile required to force the turbulent momentum transport $\left(\overline{u^{\prime} w^{\prime}}\right)$ to zero at depth. Note the general consistency between the three estimates, and the steepness of the profiles near the front. This significant drop in surface elevation, on the order of $20 \mathrm{~cm}$ across roughly $0.5 \mathrm{~km}$, contributes a significant amount of kinetic energy to the system. The unresolved dynamics limiting the size of the control volume for Pass $\mathrm{C}$ can also be seen in the divergence of the no-motion estimate and the zero-stress estimate landward of roughly $-1.4 \mathrm{~km}$.

Freshwater streamlines are used to define the bottom surface for the control volumes, as used for estimating profiles of $w_{j}$. The turbulent momentum transport is opposite in sign as compared to the mean transport, so that the total vertical momentum flux can be of either sign depending on the relative magnitude of each component. This indicates that the seaward velocities within the plume can be reduced either through the downward export of momentum, or the upward transport of fluid with less momentum.

Profiles of turbulent momentum flux, $\overline{u^{\prime} w^{\prime}}$, are shown in Figure 3.11. These profiles peak between approximately 2 and $5 \mathrm{~m}$ below the surface at values on the order of 1 to $2 \times 10^{-3} \mathrm{~m}^{2} \mathrm{~s}^{-2}$. In Figure 3.12 vertical profiles for two quantities derived from the turbulent momentum flux are shown. In the first panel, profiles of TKE production are plotted, calculated as the product of the turbulent momentum flux and the local shear:

$$
P=-\overline{u^{\prime} w^{\prime}} \frac{\partial u}{\partial z}
$$

These profiles are similar in shape to the turbulent momentum flux profiles, with production peaking at values on the order of 0.5 to $1 \times 10^{-3} \mathrm{~m}^{2} \mathrm{~s}^{-3}$. Profiles of eddy 


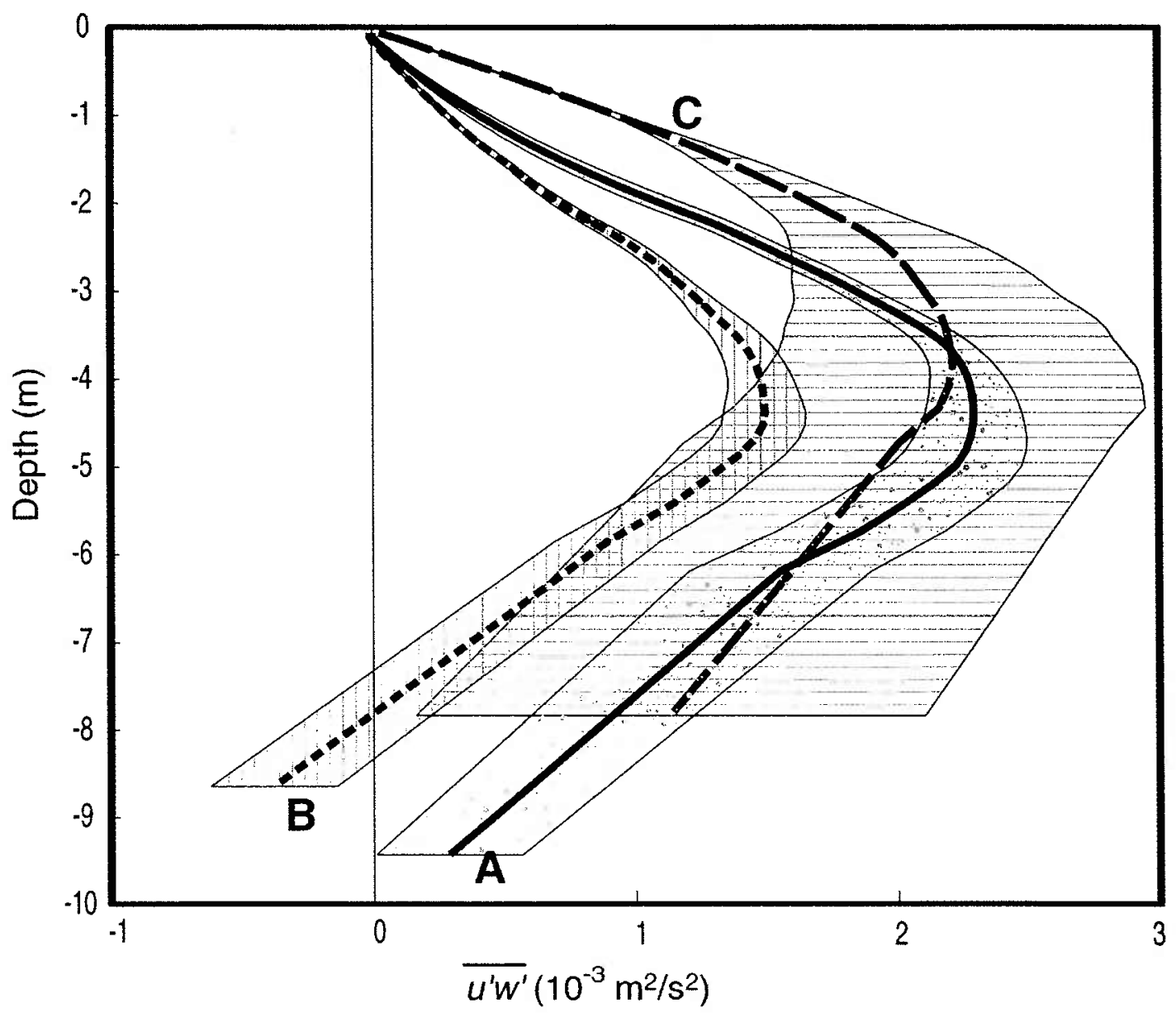

Figure 3.11: Turbulent momentum flux $\left(\overline{u^{\prime} w^{\prime}}\right)$ profiles from control volume analysis. Each estimate is bracketed by standard deviation as in Figure 3.8. Note that estimates below approximately 5 to 6 meters are influenced by unresolved lateral fluxes as discussed in the text. 

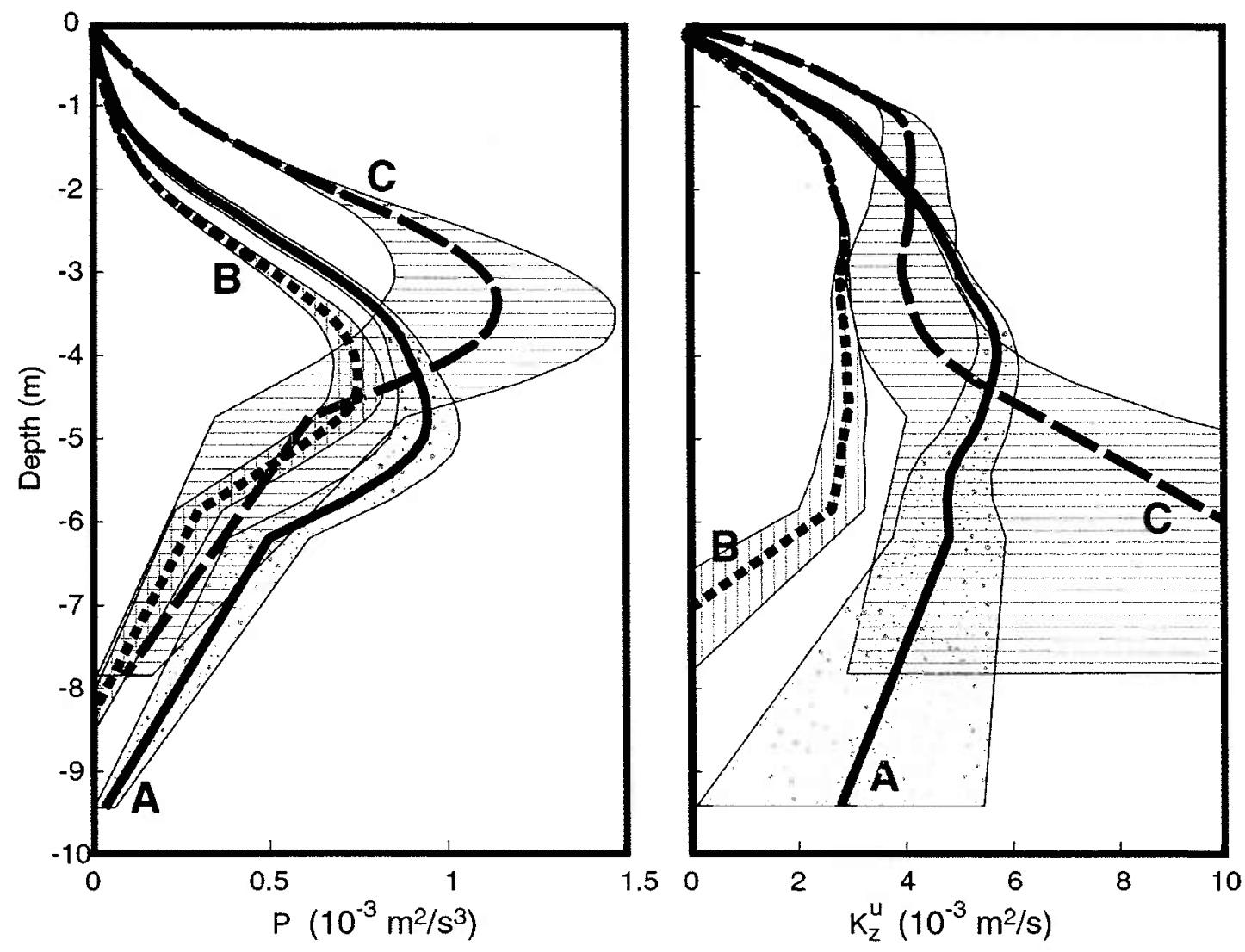

Figure 3.12: TKE production (P), and eddy viscosity $\left(K_{z}^{u}\right)$ profiles from control volume analyses. Each estimate is bracketed by standard deviation as in Figure 3.8. Note that estimates below approximately 5 to 6 meters are also influenced by unresolved lateral fluxes as discussed in the text. Eddy viscosity profiles for passes $B$ and $C$ exceed the bounding limits of the plot at depth. The scale was constrained to provide enhanced detail in the central portion of the water column. 
viscosity are shown in the second panel. These profiles are consistent in shape and magnitude with the profiles of eddy diffusivity shown in Figure 3.9, indicating that momentum and salt are diffused similarly through turbulent processes.

\subsubsection{Flux Richardson Numbers}

Estimates of the flux Richardson number, generated from the independently estimated production and buoyancy flux profiles, are plotted in Figure 3.13 against the local gradient Richardson number. Values are taken from each pass at 1 psu intervals, from 5 psu through $15 \mathrm{psu}$, representing depths between about 2.5 to $5.5 \mathrm{~m}$, so that the set of values from any particular pass are not necessarily mutually independent. The dashed line superimposed over the data, represents the simple model that $R i_{f}=R i_{g}$. As discussed earlier, this model is based on the assumption that vertical eddy diffusivities for mass and momentum are equal. Comparison of Figures 3.9 and 3.12 indicate that this assumption is reasonable in the lift-off zone. A conclusion that is consistent with the position of the dashed line in Figure 3.13 relative to the cloud of data points.

In general the results shown in Figure 3.13 indicate that most mixing in the Fraser lift-off occurs at gradient Richardson numbers slightly less than $1 / 4$, with flux Richardson numbers between 0.15 and 0.25 . These results are consistent with previous observations of highly energetic shear-induced turbulence (Gargett and Moum, 1995), and laboratory experiments and field observations at low values of $\frac{\varepsilon}{v N^{2}}$ (e.g. Ivey and Imberger, 1991; Osborn, 1980). They are not consistent, however, with measurements from gridgenerated turbulence at high values of $\frac{\varepsilon}{v N^{2}}$ (Barry et al., 2000), or observations from 


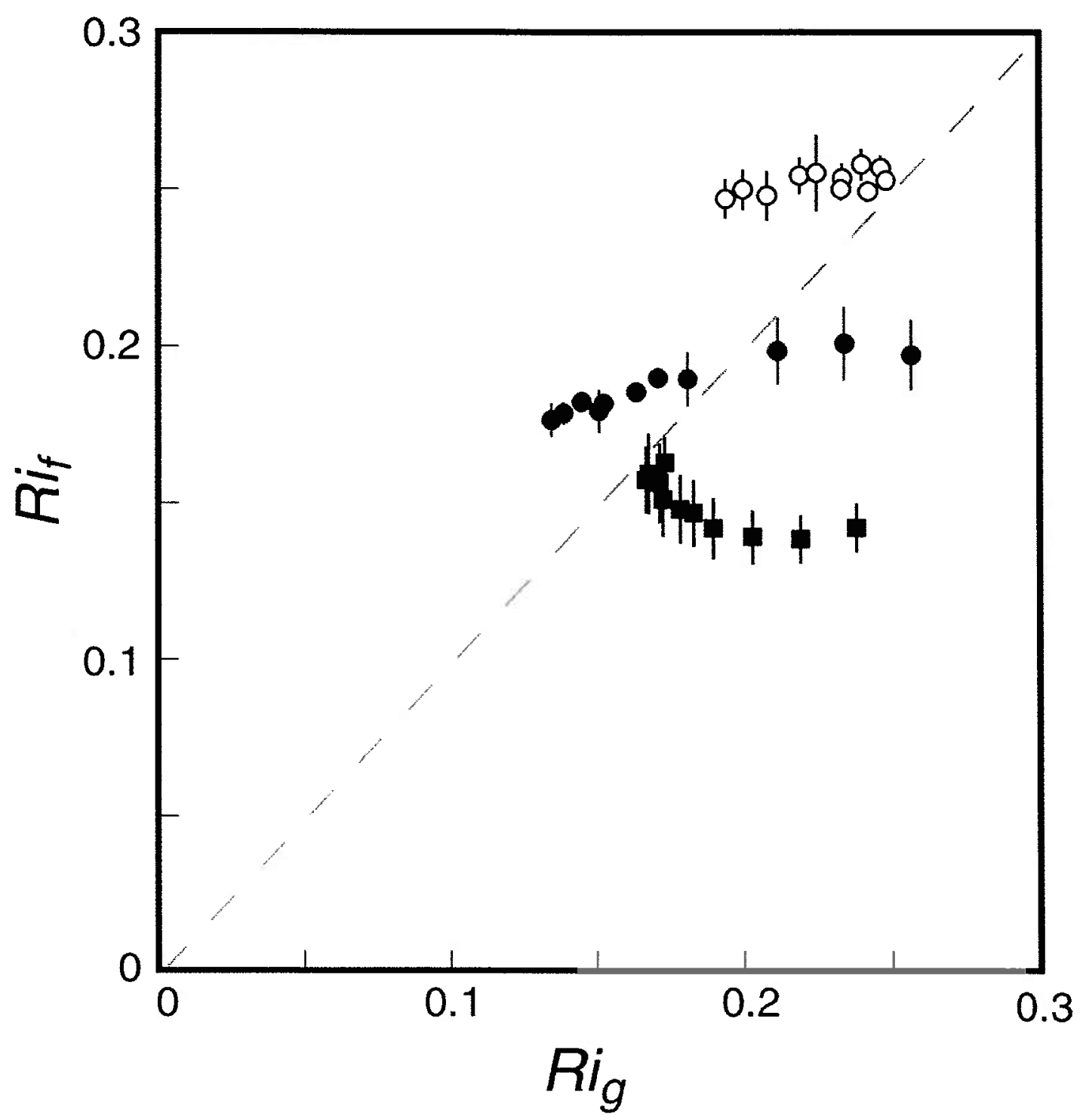

Figure 3.13: Flux Richardson number $(\mathrm{B} / \mathrm{P})$ as a function of the gradient Richardson number for Passes A (open circles), B (gray filled circles), and C (black filled squares). Points shown represent values at isohalines from 5 to 15 psu. Error bars represent standard deviation, as in Figure 3.8. The dashed line represents a 1:1 relationship between $R i_{f}$ and $R i_{g}$. 
regions where turbulence is generated by the breaking of internal waves not related to local shear (Etemad-Shahidi and Imberger, 2001). These comparisons further suggest that the magnitude of buoyancy flux is highly influenced by the nature of the mechanisms responsible for generating the turbulence.

\subsection{Lateral Effects}

In addition to measurement errors associated with the raw variables, a potential source of error to the control volume calculations is the lateral import or export of volume, salt and momentum. A separate analysis of data from the 2000 freshet, as discussed in Chapter 2, has suggested that the flow in this region of the channel is nearly two-dimensional, particularly above the $20 \mathrm{psu}$ isohaline, based on a three-dimensional salt balance and the relative contribution of lateral (southward-directed) salt flux to the overall salt budget in the region. However, due to the complexities of the interactions between entrainment and turbulence in generating the vertical transport, there are multiple pathways through which lateral fluxes may impact the calculations. An estimate of the magnitude of the contributions of lateral flux to the overall volume, momentum, and salt budgets is, therefore, an important goal.

An estimate of this magnitude can be obtained using the observed cross-stream velocity profile, and estimating a cross-channel length scale, $l_{c}$, which represents the distance to the channel boundary across which the lateral flow must decrease to zero. A 
profile of lateral volume influx can be estimated by integrating the cross-stream velocity profile:

$$
\hat{V}(h)=\int_{x_{1}}^{x_{2}} \int_{h}^{0} \nu\left(\frac{b}{l_{c}}\right) d z d x
$$

where $\hat{V}$ represents the lateral volume influx, and $v$ is the cross-stream velocity.

The first panel of Figure 3.14 shows profiles of cross-stream velocity averaged across the control volume for each of the passes. In the second panel, the ratio of lateral volume influx to the maximum flux divergence in the streamwise direction (in this case representing the lowest calculation point in the water column) is plotted. The curves in the second panel were generated using a depth dependent value of $l_{c}$ based on local bathymetry. The cross-stream velocity profiles in the first panel show relatively small and balanced velocities in the top $4 \mathrm{~m}$ of the water column, with more intense southwarddirected velocities, approaching $30 \mathrm{~cm} \cdot \mathrm{s}^{-1}$, in the lower portion of the water column. It is the flux in this lower region that supplies most of the salt to the lift-off zone, as discussed in Chapter 2, and is most likely to influence the salt balance and thus impact the turbulence calculations.

As expected, based on the velocity profiles in the first panel, the ratio in the second panel of Figure 3.14 increases rapidly below about 5 meters, with errors that are generally negligible in the upper half of the water column. The negative sign of the ratio indicates that the calculated values of $w_{h}$ and $w_{j}$ are likely to be overestimated, and that velocities may begin to decrease below about $5 \mathrm{~m}$ rather than the monotonic increase suggested by the curves in Figure 3.8 . 


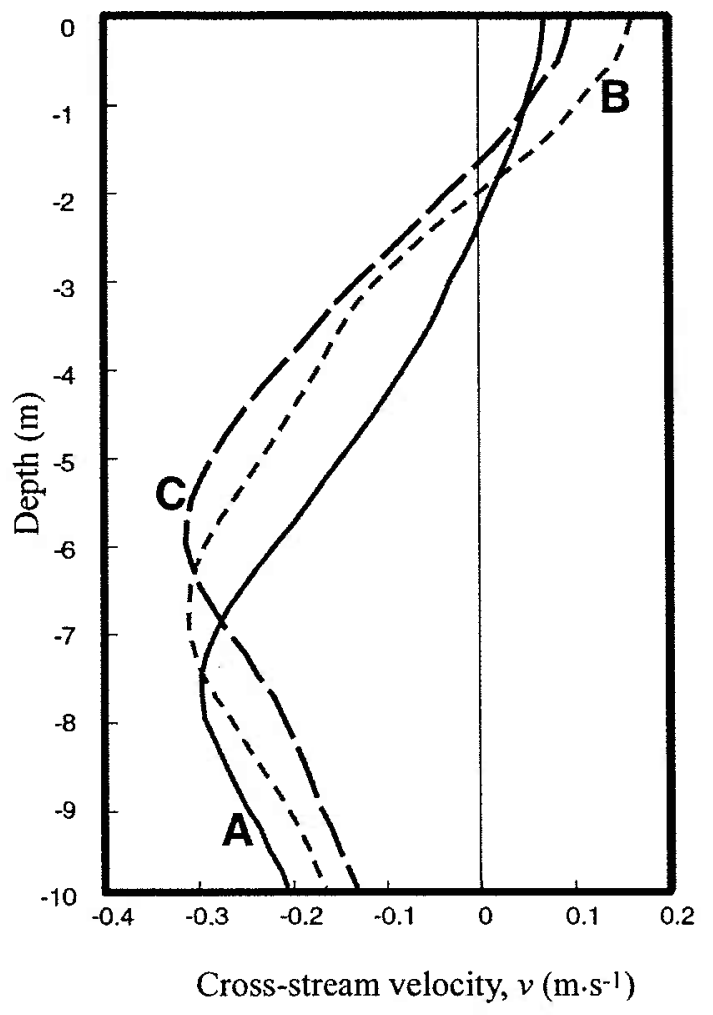

(a)

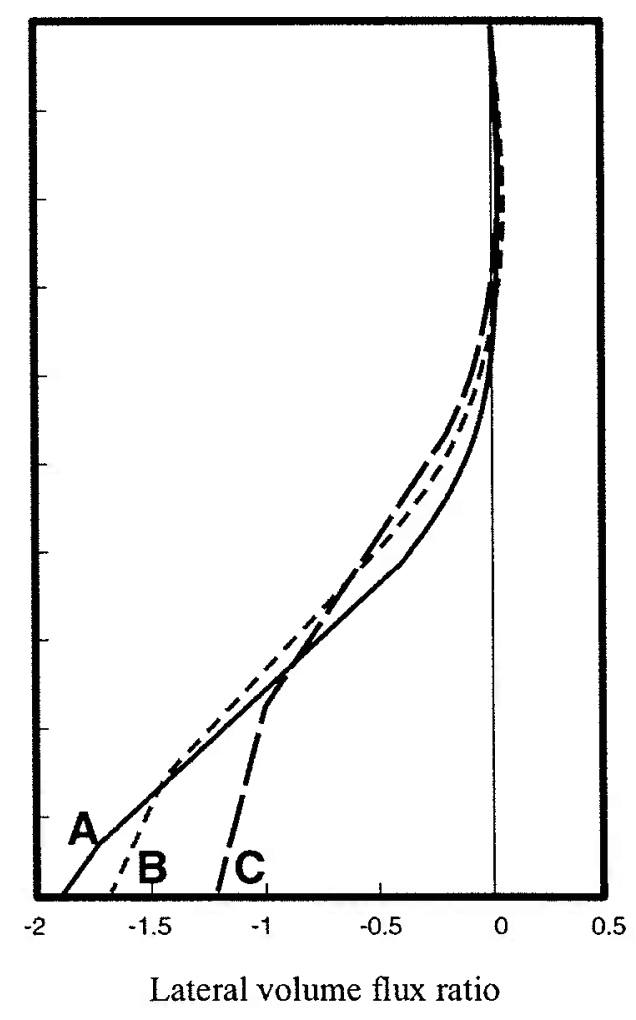

(b)

Figure 3.14: (a) Vertical profiles of the cross stream velocity, positive values directed to the right of the discharging flow (northward). (b) Profile of the estimated lateral volume flux to the maximum observed streamwise volume flux divergence, as described in the text. 
Uncertainty in the value of the vertical entrainment velocity propagates into the turbulence flux estimates directly through the mean transport terms of Equations (3.17) and (3.20), where it is amplified by the mean value of the transported quantity. Hence, potential errors in the lower portion of the water column due to errors in the vertical velocity are greatly amplified in the turbulent salt flux calculations due to the high value of salinity at depth. Errors are similarly reduced in the momentum flux calculations due to the small along-channel velocities at depth.

Additional errors are introduced to the turbulence calculations through the direct lateral flux of momentum and salt. These errors are typically of opposite sign to the entrainment related errors, reducing the overall impact of the lateral influx. Figure 3.15 presents plots similar to those shown in the second panel of Figure 3.14, but representing the net effect of lateral influx (both the entrainment and direct components) on both the salt budget and momentum budget calculations. These plots, combined with the curves presented in Figure 3.14, and the assessment of direct effects to the streamline calculations clearly indicate that a high degree of uncertainty will be associated with all calculations at depths below approximately five to six meters. Further discussion of these results will generally be limited to those within the upper region of the water column where errors associated with lateral fluxes are small. 


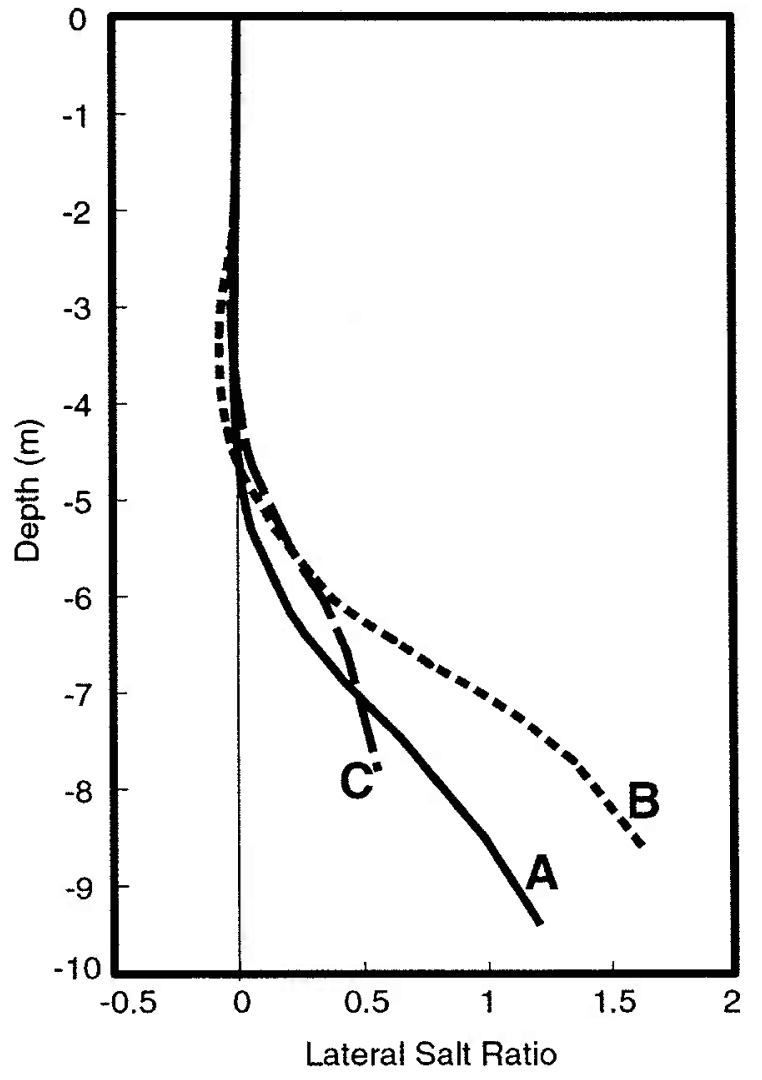

(a)

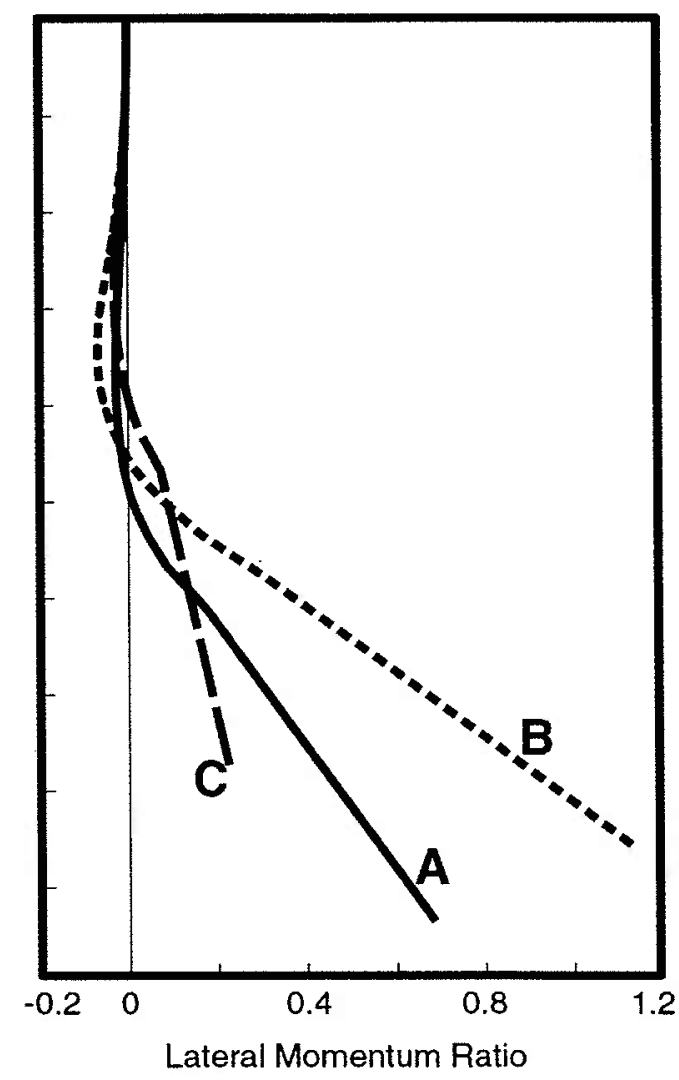

(b)

Figure 3.15: Lateral influx ratios for both salt (a) and momentum (b). The ratios represent the cumulative effect of lateral influx (including both the direct lateral flux divergence, and the indirect effect of lateral flux induced entrainment) divided by the maximum calculated values of $\overline{S^{\prime} w^{\prime}}$ and $\overline{u^{\prime} w^{\prime}}$, as shown in Figures 3.9 and 3.11. Significant errors are identifiable in both cases in the lower portion of the water column below approximately 6 meters depth, with the salt flux calculations more susceptible to lateral errors than the momentum calculations. 


\subsection{Turbulence, Entrainment and Closure}

The results shown in Figure 3.12 place the magnitude of the dissipation rate in the Fraser lift-off zone on the order of $10^{-3} \mathrm{~m}^{2} \mathrm{~s}^{-3}$. Corresponding values of $\frac{\varepsilon}{v N^{2}}$ are on the order of $10^{4}$. These TKE production rates represent a large amount of turbulent energy in comparison to other studies from ocean and coastal environments. In particular, Gregg(1989), reports that dissipation rates within the oceanic thermocline typically fall within a range of order $10^{-10}$ to $10^{-6} \mathrm{~m}^{2} \mathrm{~s}^{-3}$. Higher rates have been observed in tidal channels and in estuaries, but rarely exceeding $10^{-4} \mathrm{~m}^{2} \mathrm{~s}^{-3}$, and typically several orders of magnitude lower (see Grant, Stewart and Moillet, 1962; Gargett and Moum, 1995; and Peters,1999). For comparison, turbulent eddy diffusivities are on the order of $0.5 \times 10^{-2}$ $\mathrm{m}^{2} \mathrm{~s}^{-1}$, as shown in Figures 3.9 and 3.12. These values are more typical of observed $K_{z}$ values in ocean and coastal environments (e.g., Ledwell et al, 1993; Osborn, 1980).

A third expression of turbulent intensity, and one that can be corroborated with independent observations, is the outer length scale of the turbulence, represented by the Ozmidov scale, as in (3.9). Based on a representative dissipation rate of approximately $0.5 \times 10^{-3} \mathrm{~m}^{2} \mathrm{~s}^{-3}$, and a representative buoyancy frequency of $0.17 \mathrm{~s}^{-1}$ (equivalent to a change in salinity of 15 psu across $4 \mathrm{~m}$ ), an associated Ozmidov scale is equal to approximately $30 \mathrm{~cm}$. This scale is the scale of the largest eddies that can exist without being appreciably constrained by the stratification (e.g., Smyth and Moum, 2000).

Observations of displacement distances associated with overturns recorded in the CTD to-yo profiles (approximately 5 to $10 \mathrm{~cm}$ resolution, interpolated to $5 \mathrm{~cm}$ ), indicate 
an overturn scale of approximately $20 \mathrm{~cm}$. This scale was calculated as the rms value of all displacement values between 5 and $20 \mathrm{psu}$, including zero displacements, within the three control volumes. Maximum observed displacements were on the order of 1 to $2 \mathrm{~m}$. The outer turbulent length scale, as represented by the Ozmidov scale, is approximately $50 \%$ larger than the observed rms displacement scale. This implies that the ratio of the Ozmidov and overturn scales may not be close to one (Baumert and Peters, 2000). As discussed by Smyth and Moum (2000), there appears to be a consensus that the ratio of overturn scale to Ozmidov scale evolves in time, and that the ratio could be a useful tool for diagnostically interpreting the age of turbulence fields. However, the nature of the temporal evolution is poorly understood, to the point where there is disagreement over the direction of the evolution, from small to large values, or vice versa (Wijesekera and Dillon, 1997; Gibson, 1998). The turbulence field observed in the Fraser lift-off zone is likely to be evolving through the control volume region, and the observed overturn to Ozmidov scale ratio may be representative of this evolution.

\subsubsection{Local Production vs. Advected TKE}

The observed production in the pycnocline is on the same order as production due to near bottom boundary layer effects in the unstratified portion of the channel. Boundary layer shear production in the unstratified channel can be estimated assuming a log-layer shear profile as $P=\frac{u_{*}{ }^{3}}{\kappa z}$, where $u_{*}$ is a local shear velocity $\left(u_{*}{ }^{2} \approx C_{d} \bar{u}^{2}, C_{d}\right.$ is a bottom drag coefficient, typically on the order of $3 \times 10^{-3}$ ), $\kappa$ is von Karmans constant (equal to approximately 0.4 ), and $z$ is the distance above the bed. This suggests that the observed 
production could potentially be advected into the stratified region from the unstratified boundary layer. However, a length scale for such advection can also be estimated, assuming that advection balances the observed production, yielding an extinction length

scale, $L_{x}=\frac{\kappa z}{\sqrt{C_{d}}}$. The length scale for a representative height above the bed of $2.5 \mathrm{~m}$ is on the order of $20 \mathrm{~m}$, nearly two orders of magnitude smaller than the streamwise length scale of the lift-off region. These simple estimates indicate that the turbulent production observed in the lift-off region is produced locally, and not advected seaward from the boundary layer of the unstratified channel.

Observation of enhanced local TKE production in the liftoff zone is also consistent with the Froude number profiles shown in Figure 3.4, which imply significant energy losses through the steady decline in Froude number seaward of approximately $-1.5 \mathrm{~km}$. This general conclusion suggests that analytical (e.g., Armi and Farmer, 1986; Farmer and Armi, 1986) and numerical models (e.g., Garvine, 1982) of river plumes that incorporate inviscid conditions near the lift-off region may result in a significant overestimate of outflow velocities. An alternative scheme for parameterizing mixing and dissipation in these regions is needed. Towards that end, a discussion of the mechanisms responsible for transferring energy into the turbulence from the mean horizontal current, and the relationship of these mechanisms to bulk quantities of the flow, is undertaken in the remainder of this chapter. 


\subsubsection{Mean Vertical Velocities}

Representative values of both $w_{h}$ and $w_{j}$ near the middle of the water column are on the order of 0.1 to $0.2 \mathrm{~cm} \cdot \mathrm{s}^{-1}$, as shown in Figure 3.8. Although these velocities are small relative to the mean horizontal velocities, they provide a significant vertical transport of both salt and momentum. Vertical entrainment across density surfaces, as described by $w_{h}$, is related to gradients in mixing intensity, as discussed in Section 3.1. Entrainment into the body of the plume, represented by $w_{j}$, is augmented by a flow divergence in the plume interior due to the seaward acceleration of ambient Strait of Georgia water through turbulent processes.

The ratio of the observed entrainment velocity to a representative upper layer velocity is analagous to the entrainment coefficient, $E$, defined by Ellison and Turner (1959), and is on the order of $1 \times 10^{-3}$ in the Fraser lift-off region. This value is roughly an order of magnitude smaller than values of $E$ predicted by Ellison and Turner based on laboratory experiments of plumes. Ellison and Turner suggested that entrainment should be a function of a bulk Richardson number, $R i_{B}=\frac{g^{\prime} H}{U^{2}} \cos \alpha$, where $H$ and $\mathrm{U}$ are the length and velocity scales of the flow, respectively, and $\alpha$ is the bottom slope. This bulk Richardson number is equivalent to the inverse square of the layer Froude number introduced in Chapter 1, and plotted for the three lift-off passes in Figure 3.4. The Froude numbers in Figure 3.4 correspond to bulk Richardson numbers of order 0.5 to 1 , with an average value on the order of 0.7 . As the plume in this case is surface attached, $\alpha$ would represent the surface slope, which can be considered equal to zero. 
Although the results of Ellison and Turner did not include bulk Richardson numbers as high as the range present in the Fraser lift-off, their data suggest a decreasing trend in $E$ with increasing $R i_{B}$, towards values consistent with the present observations. The results of other laboratory studies, as compiled by Christodoulou (1986), confirm the trend suggested by the data of Ellison and Turner, extending the range of observations to values of the bulk Richardson number as high as $10^{2}$. This expanded data set suggests a value of the entrainment coefficient on the order of $10^{-3}$ for $R i_{B} \sim 1$, which is consistent with the present observations. In situations where flow conditions continuously adjust to drive Froude numbers towards unity, which appears to be the case in the Fraser lift-off, the entrainment coefficient would be approximately constant at this value.

\subsubsection{Mechanisms of Turbulence Generation}

The most likely mechansim for the input of energy from the mean horizontal flow into turbulence is the generation of Kelvin-Helmholz billows through the propagation of shear instabilities. The development and collapse of these instabilities in a laboratory flow similar to the Fraser lift-off is well described by Pawlak and Armi (2000).

Equation (3.10) presented scaling relationships for dissipation and mixed layer thickness, using the two independent variables in a shear stratified flow, $\Delta u$ and $g^{\prime}$. Based on the velocity and salinity observations shown in Figure 3.6, representative values of $\Delta u$ and $g^{\prime}$ for the Fraser liftoff are approximately $2 \mathrm{~m} \cdot \mathrm{s}^{-1}$, and $0.15 \mathrm{~m} \cdot \mathrm{s}^{-2}$, respectively. Using these values, and following (3.10), the coefficient $\varphi$, which represents the ratio of the mixed layer thickness, $\delta$, to a length scale generated from $\Delta u$ and $g^{\prime}$, is on the order of 0.2 to 0.25 , consistent with subcritical values of $R i_{g}$. In fact, 
substitution of the second equation in (3.10) into the definition of the gradient Richardson number yields the same result:

$$
\delta=R i_{g}\left[\frac{(\Delta u)^{2}}{g^{\prime}}\right]
$$

This indicates that the coefficient $\varphi$ in (3.10) is equal to $R i_{g}$. Because stability issues and energy considerations (Miles, 1961; Howard, 1961; Thorpe, 1973) tend to result in a relatively constant value for the gradient Richardson number in regions of active turbulence, the mixed layer thickness, $\delta$, is not an independent variable in this type of system.

The value of the coefficient $\gamma$ in (3.10), which represents the ratio of the observed dissipation rate to a scaled dissipation rate, is on the order of $2 \times 10^{-3}$.

$$
\gamma=\frac{\varepsilon}{\Delta u g^{\prime}} \sim 2 \times 10^{-3}
$$

It can be shown that this coefficient, $\gamma$, is proportional to an interfacial drag coefficient, $C_{D i}$, divided by the gradient Richardson number,

$$
\gamma=\left(1-R i_{f}\right) \frac{C_{D i}}{R i_{g}}
$$

suggesting a value of the interfacial drag coefficient on the order of $5 \times 10^{-4}$. Both the drag coefficient and the coefficient $\gamma$ relate to the efficiency with which energy is extracted from the mean flow and converted into turbulent energy, suggesting that the turbulent energy is on the order of three orders of magnitude lower than the mean flow energy. Because turbulence and entrainment are related phenomena, as discussed in 
Section 3.1, there may be a dependence of the drag coefficient on the bulk Richardson number, similar to the relationship that appears to hold for the entrainment coefficient (e.g., Ellison and Turner, 1959; Christodoulou, 1986). In addition, the interfacial drag coefficient may be constrained by lateral influences affecting the plume expansion rate. In a dynamic system controlled by conservation of volume, mass, and momentum, and a mixing threshold associated with the gradient Richardson number, the drag coefficient could also be affected by limitations on the plume width and expansion. Such limitations could be imposed by local geometry, or ambient conditions in the receiving waters.

The use of these scaling relationships in context with observed turbulent quantities requires the assumption that the turbulence in the shear stratified layer is fully evolved. As discussed in context with the discrepency between the overturn and Ozmidov scales, it is unclear how fully developed the turbulence in the Fraser lift-off is. Observed values of the appropriate coefficients are presented here, however, for future comparison with observations from other similar shear stratified environments.

\subsection{Concluding Remarks on Mixing in the Lift-Off Zone}

This chapter has provided a robust control volume approach for estimating TKE quantities in a shear-stratified flow. The resulting estimates of turbulent dissipation, on the order of $10^{-3} \mathrm{~m}^{2} \mathrm{~s}^{-3}$, are high compared to most other observations of dissipation in oceanic and coastal environments. These results are reasonable, however, in light of the fact that there are only two independent variables in a shear-stratified flow, $\Delta u$ and $g^{\prime}$. In the Fraser lift-off both of these independent variables are high, at $2 \mathrm{~m} \cdot \mathrm{s}^{-1}$ and 
$0.15 \mathrm{~m} \cdot \mathrm{s}^{-2}$, respectively. The high values of dissipation observed in the lift-off region are consistent, as dissipation must scale with the product of these two variables.

Further contributions of this chapter are based on the results of these turbulent calculations, and the highly energetic setting of the study site, which is characterized by values of $\frac{\varepsilon}{v N^{2}}$ on the order of $10^{4}$. First, mixing efficiencies, as expressed by the flux Richardson number, were observed to be consistent with previous laboratory and field observations representing far less energetic conditions (Ivey and Imberger, 1991;Osborn, 1980). Specifically, it was seen that most mixing occurred at gradient Richardson numbers between 0.15 and 0.25 , with flux Richardson values within the same range. The entrainment coefficient for the flow was found to be on the order of $10^{-3}$, which is not inconsistent with trends suggested by previous studies (Christodoulou, 1986). Due to feedback mechanisms that tend to keep the Froude number and bulk Richardson number of a plume close to unity, the entrainment coefficient is expected to be relatively constant for a wide range of estuarine plumes. This is also suggested by the relative dynamical simplicity of a fully developed shear-stratified flow, which can be adequately characterized by only two independent variables, $\Delta u$ and $g^{\prime}$. In the same vain, the issue of turbulence closure was discussed with respect to these two independent variables, resulting in the formulation of a drag coefficient for the flow on the order of $C_{D i} \sim 5 \times 10^{-4}$. The combination of these simple general expressions describing entrainment and turbulence in the Fraser lift-off region may prove useful for predicting flow evolution for a wide variety of shear-stratified flows based on mean flow properties. 


\section{Chapter 4}

\section{The Variability of Vertical Salt Flux in a Highly Stratified Estuarine Channel}




\begin{abstract}
The temporal and spatial variability of vertical salt flux in the dynamics of the highly stratified Fraser River Estuary, British Columbia, was investigated observationally, using several different direct and indirect indicators of buoyancy flux. Data were collected from the estuary using shipboard instrumentation, primarily an acoustic Doppler current profiler (ADCP), and a towed conductivity, temperature, depth (CTD) unit. Estimates of buoyancy flux were made from along channel control volume analyses and from measurements of overturn scales in the vertical salinity profiles. The temporal evolution of the salt wedge structure through a tidal cycle was evaluated using the results of these buoyancy flux calculations, as well as gradient Richardson number, Froude number, and stratification profiles.

Vertical salt flux, as opposed to seaward advection of high salinity fluid, was found to be the dominant mechanism responsible for removal of salt from the estuarine channel during each tidal cycle. Buoyancy flux was highly variable in time and space, however, with vertical salt flux during ebb tides on the order of 2 to 3 times greater than that occurring during floods. This is due to an increase in vertical velocity shear and a sharp increase in stratification, which was found to occur during early ebb. Mixing during all phases of the tide was considered significant, however. Enhanced mixing was observed spatially within a region dominated by a channel constriction in which the channel narrows by approximately $25 \%$.
\end{abstract}




\subsection{Introduction}

Estuaries are an important component of the coastal ocean, providing nutrient-rich waters that form the foundation of fertile and productive ecosystems. In the coastal ocean, estuaries continue to play an important role, with a significant impact on the distribution of land-derived nutrients, contaminants and sediment. These far field distributions are driven to a large extent by localized mixing processes within an estuarine channel, where the confluence of energy from river and tidal sources can generate sufficient turbulence to overcome local stratification. Early studies of estuarine physics (e.g., Schijf and Schonfeld, 1953; Pritchard, 1952, 1954, 1956) recognized estuarine mixing as an important component of estuarine circulation. However, even today, attempts to quantify mixing rates continue to represent a major technical challenge.

Mixing in estuaries can be highly variable, dependent on diurnal and fortnightly variations in tides, seasonal cycles of river discharge and variations in channel topography. Simpson et al. (1990) found that in the partially mixed estuary of Liverpool Bay, intense mixing occurs primarily near the end of the flood, particularly near spring tides. Recent measurements of mixing in the Hudson River Estuary (Peters, 1999), another partially mixed estuary, indicate that about $30 \%$ of the total fortnightly vertical salt flux occurs during spring ebbs, with the majority of the remainder provided during floods throughout the fortnightly cycle. A recent numerical study of mixing in a partially mixed estuary (MacCready and Geyer, 2001) indicates that mixing is most intense during the peak ebb, but more vertical salt flux occurs during the peak flood due to an extended 
along-channel length of the isopycnals. In the Tacoma Narrows section of Puget Sound, a large fjord-like estuary, channel curvature and the flow dynamics over a sill were found to produce strong vertical mixing (Seim and Gregg, 1997), reiterating the often observed importance of topographic features to mixing processes (e.g., Geyer and Canon, 1982; Farmer and Armi, 1999; Wesson and Gregg, 1994).

While significant progress has been made in identifying mixing in different estuarine environments, fundamental questions still remain regarding the mechanisms responsible for mixing such as where and when the mixing actually occurs, and how these processes are dynamically controlled. Conditions in highly stratified estuaries may be fundamentally different than in other types of estuaries. For instance, steep density gradients can provide an increased resistance to mixing. However, these steep gradients may also result in more productive vertical salt flux once the turbulence is energetic enough to overcome the stratification.

Several previous studies have described the mixing climate of the highly stratified Fraser River (Geyer and Smith, 1987; Geyer, 1988; Geyer and Farmer, 1989). These studies have suggested that mixing is more prominent in the Fraser during ebbs, and at localized constrictions in the channel width (Geyer, 1985), but no attempts to directly assess mixing rates within the estuary were attempted. It is still unclear to what degree mixing on the flood is significant in natural salt-wedge systems, and, specifically with regards to the Fraser River Estuary, how much mixing actually occurs during a typical tidal cycle, and where and when that mixing is most intense. 
This chapter focuses on data from a cruise in the Fraser River Estuary during the summer of 2000. As discussed in Chapter 1, the Fraser Estuary is a highly energetic salt wedge estuary, characterized by a strongly diurnal tide, with amplitudes exceeding $4 \mathrm{~m}$ during spring tides, and a river discharge that can peak at over $10,000 \mathrm{~m}^{3} \mathrm{~s}^{-1}$ during the summer freshet. These conditions combine to generate a salt wedge that advances into the channel some 15 to $20 \mathrm{~km}$ landward of the mouth during the flood portion of every tidal cycle, retreating to the mouth once a day during the largest of the two daily ebbs. The dates of the 2000 cruise were chosen to coincide with spring tides and the summer freshet, in order to observe the oscillating salt wedge under maximal energy conditions.

The objectives of understanding the dynamics of the estuarine circulation within the channel are addressed in this chapter by focusing on the key processes of shear induced mixing, the straining of isopycnals by velocity shear, and advection. The interaction of these processes within the Fraser estuary is responsible for setting and maintaining the degree of stratification within the estuary. A specific goal of the chapter is to address temporal variations in stratification and diapycnal mixing through the tidal cycle, particularly the relative strength of mixing on both the flood and ebb portions of the tidal cycle. A second and related goal is to determine the dominant processes responsible for the evacuation of salt from the channel during the ebb; that is, horizontal advection driven by tidal oscillation, or vertical mixing and subsequent transport in the upper water column. Spatial variations in mixing intensity will also be evaluated, both with respect to their importance to the temporal variability, and to previous observations of localized mixing events at channel constrictions (Geyer, 1985). 


\subsection{The Mechanics of Shear-Induced Mixing}

There are several important concepts which are useful in framing any discussion regarding turbulence and mixing. Many of these concepts have been introduced in earlier chapters of this thesis, but are briefly re-introduced here to provide a context for observations from the estuarine channel of the Fraser River.

\subsubsection{Gradient Richardson Number}

The gradient Richardson number was originally introduced in Chapter 1. It represents a comparison of the strength of the local buoyancy gradient to the strength of the local velocity gradient:

$$
R i_{g}=\frac{-\frac{g}{\rho} \frac{\partial \rho}{\partial z}}{\left(\frac{\partial u}{\partial z}\right)^{2}}
$$

where $\rho$ represents density, $u$ the fluid velocity, $g$ gravitational acceleration, and $z$ is the vertical coordinate. Flows with Richardson numbers below a critical value of $1 / 4$ are generally believed to be capable of sustaining interfacial instabilities and vertical mixing. Miles (1961) and Howard (1961) showed analytically that a subcritical gradient Richardson number was a necessary, but not sufficient, condition for the generation of turbulence. Many subsequent observations and experiments have suggested that a subcritical Richardson number can be a robust indicator for turbulent mixing (e.g., Thorpe, 1973). 


\subsubsection{Froude Numbers and Hydraulic Theory}

The Froude number represents the ratio of fluid speed to local wave speed. In density stratified fluids, the internal wave speed is of primary importance, and a composite Froude number, $G$, can be defined, as in Chapter 1:

$$
G^{2}=F_{1}^{2}+F_{2}^{2}, \quad F_{j}^{2}=\frac{u_{j}^{2}}{g^{\prime} h_{j}}
$$

where the subscripts 1 and 2 refer to the upper and lower layers, respectively, $F_{j}$ is the layer Froude number, $h_{j}$ is the layer thickness, and $g^{\prime}=g\left(\rho_{1}-\rho_{2}\right) \rho_{\mathrm{o}}^{-1}$ is a reduced gravity. The importance of the Froude number with respect to hydraulic control at an arrested front was discussed in Chapter 2, and the importance of the Froude number to turbulent mixing was discussed in Chapter 3. Here, the Froude number will be used in conjunction with the Richardson number and other parameters, to develop an understanding of the importance of specific physical processes to the density structure observed in the estuarine channel.

\subsubsection{Tubulent Salt Transport and Buoyancy Flux}

The rate of buoyancy flux provides a direct measure of the vertical flux of density difference. In regimes where density is dominated by salinity, such as the Fraser River Estuary, it represents the magnitude of vertical salt flux:

$$
B=\frac{g}{\rho} \overline{\rho^{\prime} w^{\prime}}=g \beta \overline{S^{\prime} w^{\prime}}
$$

where S represents and salinity, and $\beta=\frac{1}{\rho} \frac{\partial \rho}{\partial S}=0.77 \times 10^{-3} \mathrm{psu}^{-1}$, for temperatures near $10^{\circ} \mathrm{C}$. Buoyancy flux provides a useful measure of mixing intensity in terms of energy, 
and represents the buoyant production term in the one-dimensional turbulent kinetic energy (TKE) equation, which was introduced in Chapter 3 as equation (3.1).

Two other parameters often used in discussion of mixing intensity are the turbulent eddy diffusivity $K_{z}^{\rho}$, and a representative entrainment velocity scale, $w_{t}$. The turbulent eddy diffusivity, which represents the rate of mixing relative to a spatial gradient, can be related to buoyancy flux as (Osborn, 1980):

$$
K_{z}^{\rho}=\frac{B}{N^{2}}
$$

where $\mathrm{N}$ is the buoyancy frequency, described by:

$$
N^{2}=\frac{-g}{\rho} \frac{\partial \rho}{\partial z}
$$

A representative entrainment velocity scale, that is similar in magnitude to the diahaline velocity presented in Chapter 3 can be written as

$$
w_{t}=\frac{B}{g^{\prime}}
$$

This scale represents the vertical velocity that would be required to deliver a specified salt flux across an isopyenal.

\subsubsection{Overturn Scales}

The overturn scale, $L_{t}$, was introduced in Chapter 3 as an observational tool for identifying the outer length scales of stratified turbulence. As described by Thorpe (1977), it is equal to the rms value of the observed vertical displacement distances within an observed density profile as compared to its stably sorted counterpart. The outer scales of the turbulence can also be represented by the Ozmidov scale, $L_{o}$, which can be scaled 
directly from turbulent quantities. This scale is interpreted as the maximum vertical scale that can be achieved by an eddy given the ambient stratification and rate of turbulent kinetic energy (TKE) dissipation, $\varepsilon$, and is defined as $L_{O}=\left(\varepsilon / N^{3}\right)^{\frac{1}{2}}$ (Peters et al, 1988). Thorpe (1977) initially proposed that his measurement of the overturn scale should be related to the Ozmidov scale, and several investigations as to the nature of that relationship have been conducted since. Dillon (1982) found a ratio $\left(L_{t} / L_{O}\right)$ equal to 0.8 deep within the interior of an actively mixing surface layer, but speculated that the value of this ratio was a function of the local gradient Richardson number. Other studies have yielded values from 0.66 (Crawford, 1986) to 0.95 (Ferron et al., 1998), primarily based on oceanic measurements. Wijesekera et al (1993), and Baumert and Peters (2000), have both suggested relationships between $R i_{g}$ and $\mathrm{L}_{\mathrm{t}} / \mathrm{L}_{\mathrm{O}}$. Baumert and Peters' relationship is of the form

$$
\frac{L_{t}}{L_{O}}=3.6 R i_{g}^{3 / 4}
$$

based on a two-equation turbulence model, and supported by field data, which, while widely scattered, supports the general trend. Using this formulation, an overturn scaleOzmidov scale ratio of approximately 1.27 is consistent with a critical Richardson number value of 0.25 , and equality between the two length scales would be found at a value of $R i_{g}=0.18$. Additionally, field observations (Wijesekera and Dillon, 1997), and numerical simulations (Smyth and Moum, 2000) indicate that the ratio in (4.7) may decrease over time as shear induced turbulence ages, from values on the order of 8 down to values on the order of 0.2 . Although it appears that the $L_{t} / L_{O}$ ratio is generally of 
order 1 , a more precise value is difficult to constrain, as it is highly dependent on the Richardson number, and perhaps other parameters.

Equivalency between these two length scales will be assumed for purposes of estimating buoyancy flux in this study, allowing the easily obtained overturn scale to serve as a reasonable estimator for mixing intensity. An expression for buoyancy flux in terms of the Ozmidov scale, can be written as:

$$
B_{t}=\left(\frac{R i_{f}}{1-R i_{f}}\right) L_{O}{ }^{2} N^{3}
$$

where $R i_{f}$ is the flux Richardson number, representing the ratio of the buoyancy flux and shear production terms in the TKE equation, as discussed in Chapter 3. The analyses undertaken in Chapter 3 indicated that values of $R i_{f}$ in the highly energetic lift-off zone at the river mouth are consistent with previous laboratory studies (e.g., Ivey and Imberger, 1991), at values between 0.15 and 0.2 .

\subsubsection{Stratification}

Simpson et al. (1990) proposed that the degree of stratification in an estuary is established primarily by the interaction of two competing mechanisms: the stratifying effects of velocity-induced straining, and the homogenizing effects of shear-induced mixing. Simpson et al. (1990) quantify stratification by the amount of energy input needed to homogenize a vertical density profile. While this method is useful in some respects, it can often be misleading, particularly in areas of intense interfacial wave activity, as its value changes depending on the vertical location of the layer interface relative to mid-depth. 
Here, stratification will instead be gauged by a non-dimensional representation of mixed layer thickness:

$$
\psi=\frac{1}{2} \frac{h}{L_{50}}, L_{50}=z_{75}-z_{25}
$$

where $h$ represents the local water column depth, and $L_{50}$ represents the vertical distance between the $75^{\text {th }}\left(z_{75}\right)$ and $25^{\text {th }}\left(z_{25}\right)$ percentiles of the system salinity range. In the Fraser system, where salinities typically range from 0 to $28 \mathrm{psu}, L_{50}$ at any location would be the vertical distance between the 7 and 21 psu isohalines. If both isohalines are not observed at a given location, the value of $L_{50}$ can be estimated based on observable salinity gradients, thus allowing values of $L_{50}$ exceeding $h$ or values of $\psi$ less than 0.5 . The dimensionless stratification parameter, $\psi$, can be interpreted as a continuum from well mixed conditions at $\psi<<1$ to highly stratified conditions at $\psi>>1$.

By analogy with the model of Simpson et al. (1990), the temporal change in the dimensionless stratification parameter can be related to the sum of a straining term, which varies as $\Delta u$, and a mixing term, which varies as $(\Delta u)^{3}$, where $\Delta u$ can be taken as the velocity difference between an upper and lower layer. The derivation of these terms is described more thoroughly in the Appendix.

\subsection{The Fraser River Estuary}

The Fraser River Estuary provides an excellent location to investigate the dynamics of two layer flows, due to strong barotropic and baroclinic forcing mechanisms and periods of intense stratification. The data utilized in this chapter was collected between 
June 30 and July 4,2000. As discussed more thoroughly in Chapter 1, this sampling period was centered around the spring tide, which occurred on July $2^{\text {nd }}$, with a tidal amplitude of $4.25 \mathrm{~m}$, and was characterized by river discharges on the order of $7,000 \mathrm{~m}^{3} \mathrm{~s}-$

1. The analyses detailed in this chapter often rely on the assumption that the dynamics of the salt wedge are similar across all of the sampling days. This assumption is reasonable based on the small variations in tidal amplitude and river discharge observed throughout the period.

Data were collected from shipboard instrumentation, primarily two hull-mounted Acoustic Doppler Current Profilers (ADCPs), operating at $1200 \mathrm{kHz}$ and $300 \mathrm{kHz}$, and a towed Ocean Sensors 200 Series conductivity, temperature, depth (CTD) unit. The data discussed in this chapter was collected primarily at an anchor station located approximately $3.4 \mathrm{~km}$ landward of Sand Heads, and from channel operations located landward of the anchor station. The lower $18 \mathrm{~km}$ of the channel are shown in Figure 4.1, with the location of the anchor station highlighted by the black triangle.

As an example of the structure of the salt wedge, two composite profiles of the salt wedge, compiled from data collected during the ebb from four days of operations in the channel, are shown in Figure 4.2. The earlier of the two profiles (2.3 hours after high tide) represents a portion of the salt wedge, from the anchor station location, landward through the bend, nearly to the entrance to Steveston Harbor, but does not include the head of the salt wedge. Contours showing the salinity distribution of this profile are shown in Figure 4.2(a). The second profile, for which the salinity distribution is shown in Figure 4.2(b), represents conditions 1 hour later and is more complete, extending from 

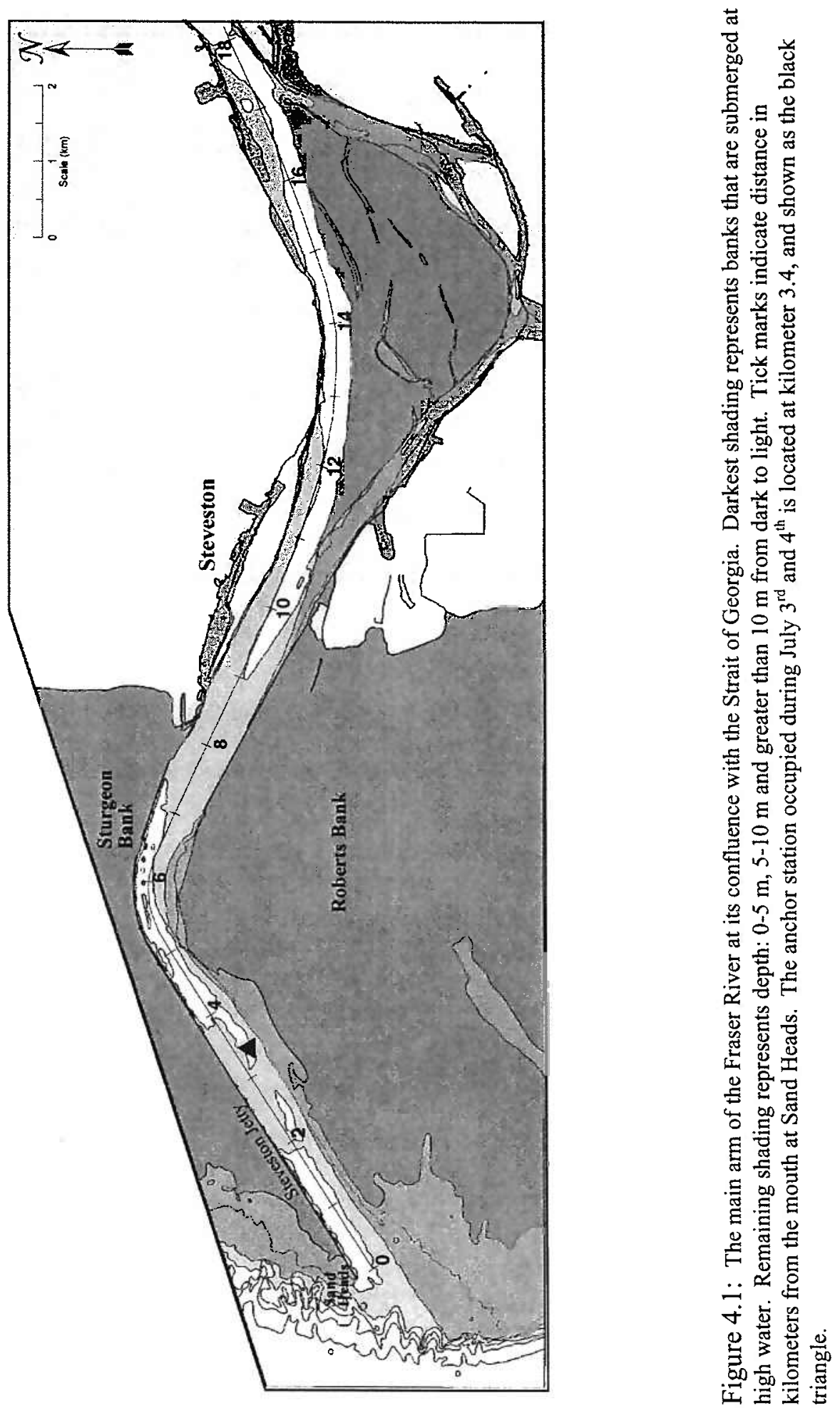


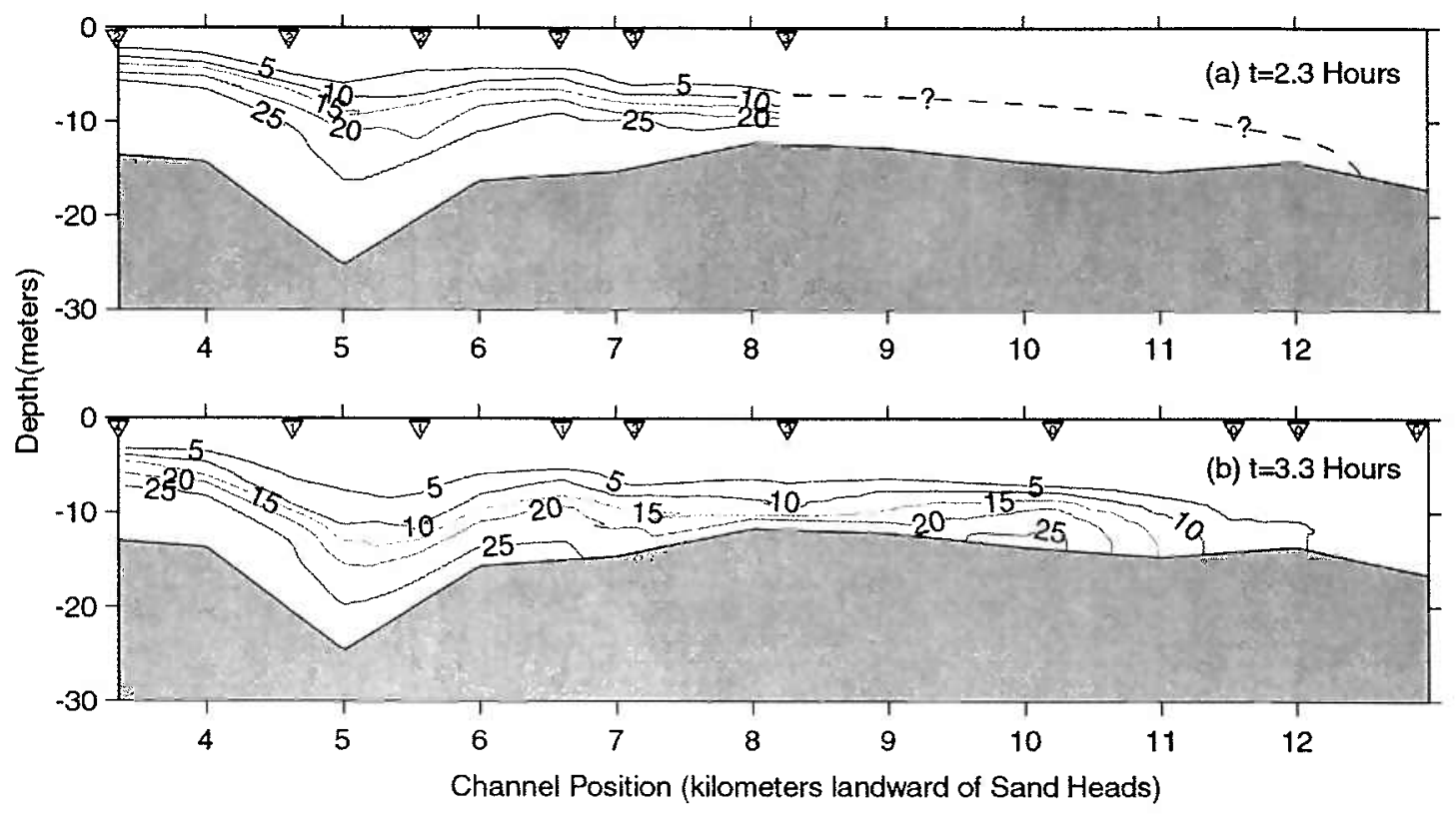

Figure 4.2: Composite profile of salt wedge salinity structure at 2.3 hours (top panel) and 3.3 hours (bottom panel) after high tide prior to the largest of two daily ebbs. Cast locations are indicated by the inverted triangles at the top of each panel. Numbers inside the triangles identify the date of the cast $[0$ June 30; 1- July1; 2- July 2; 3- July 3; 4- July 4 (Time Series Data)]. Contours are based on an interpolation scheme normalized by channel depth. 
the anchor station location landward past the head of the salt wedge, which was located approximately $10.5 \mathrm{~km}$ from the mouth.

\subsection{Data Collection During Freshet, Spring Tide Conditions}

\subsubsection{Integrated Observations From a Down-Stream Time Series}

Data were collected through a complete tidal cycle on July $3^{\text {rd }}$ and $4^{\text {th }}$, from the anchor station indicated on Figure 4.1, A CTD cast was performed approximately every 15 minutes, with both ADCPs running continuously, providing good temporal resolution across the 18 hour period during which the presence of salt was observed. Tidal stage during the anchored period is plotted with near-surface and near-bottom velocities in Figure 4.3. For convenience in comparing data from different days, all times will be referenced to the high tide leading the larger of the two daily ebbs.

The conditions observed at the anchor station location through the time series are shown in Figure 4.4, where the salinity structure is plotted, allowing an overview of the conditions through the tidal cycle. Well mixed regions forming the bulk of the upper and lower layers can be clearly seen in Figure 4.4, separated by a pycnocline, which varies in position and intensity through the tidal cycle. The dark shaded region superimposed over the salinity contours represents the regions where the gradient Richardson number, $R i_{g}$, is below $1 / 4$. The dashed line represents a contour of zero velocity. Velocities above this line are directed seaward, while regions below the line are flowing landward. The hatched region in Figure 4.4 represents the region of positive velocity shear. Within this region, velocities increase in the landward direction as one moves away from the channel 


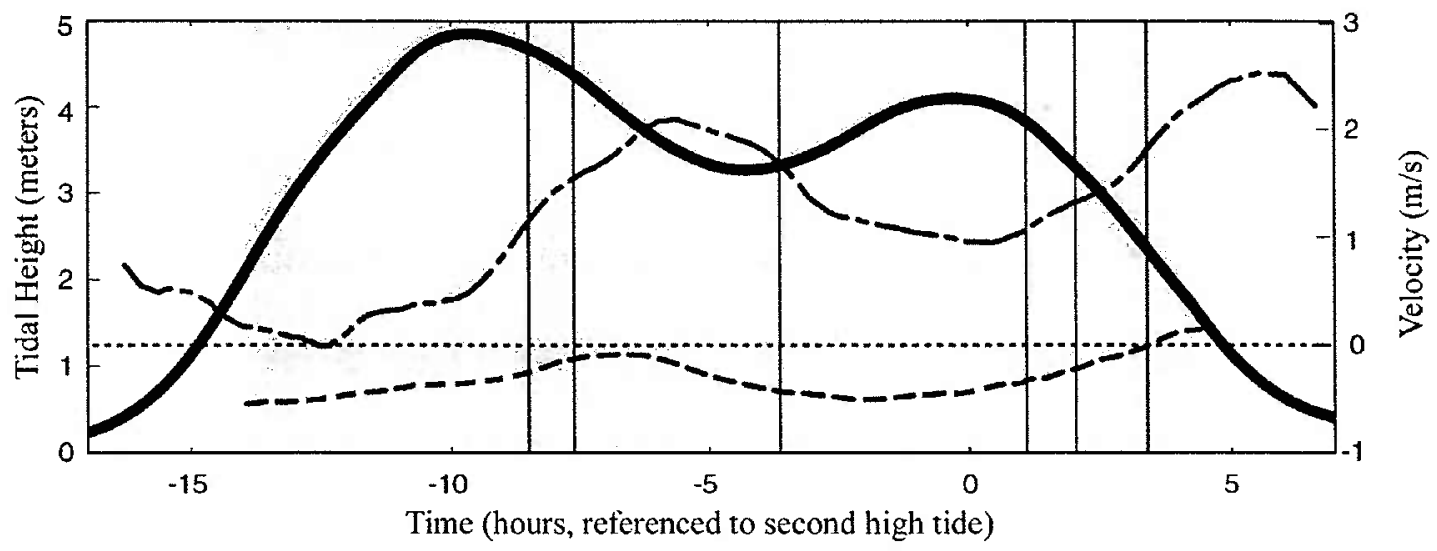

Figure 4.3: Tidal height (bold line), velocity $1.74 \mathrm{~m}$ below the surface (dash dot line, positive seaward), and velocity $2 \mathrm{~m}$ above the bed (dashed line, positive seaward) during occupancy of anchor station. Shaded region indicates presence of salt at anchor station. Vertical lines are for reference in comparison with other figures. Time is referenced to the second high tide. 


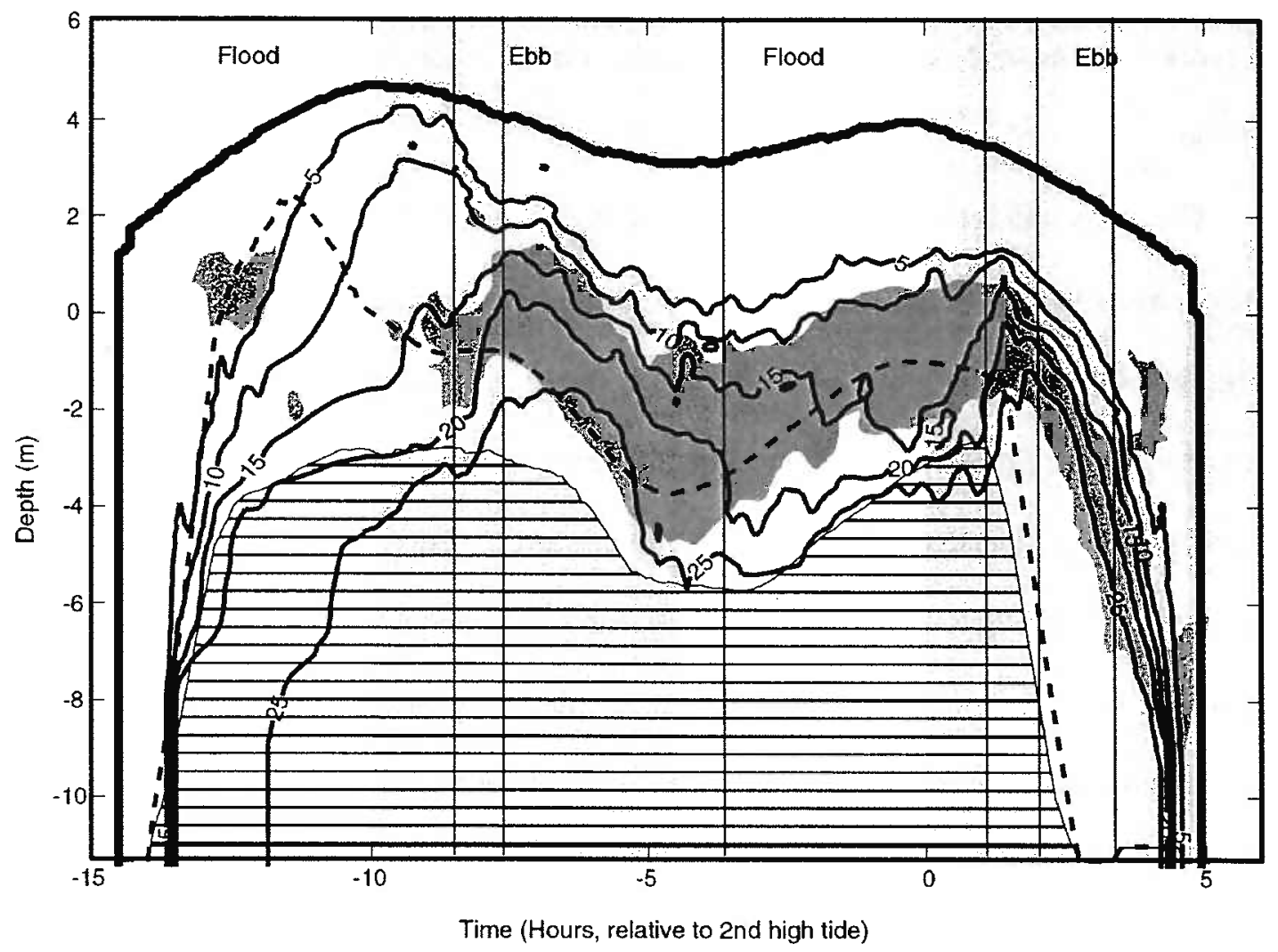

Figure 4.4: Contours of salinity (psu) as a function of depth and time through a tidal cycle at the anchor station. Light shaded areas represent ebb tides. Dark shaded area indicates $R i_{g}<1 / 4$. Dashed line represents the vertical location of $u=0$ (i.e., regions above this line are flowing seaward, and regions below the line are flowing landward). Hatched area represents the region of positive shear (i.e., velocity increasing in the landward direction away from the bed). The heavyweight line indicates the limits of the data and the surface elevation through the tidal cycle. Vertical lines are provided for reference in comparison with other figures. 
bed. Geyer and Farmer (1989) suggest that shear is most intense, and mixing most active at times when no such region exists, so that the bottom shear and interfacial shear act in unison.

The integrated influence of mixing within the estuary can be assessed by comparing the distribution of the tidal-cycle integrated landward and seaward directed salt fluxes, with respect to salinity. Such a distribution is shown by the composite bar graph in Figure 4.5, where positive values of salt mass represent landward-directed motion, and negative values represent a flux of salt in the seaward direction. Salt flux was calculated incrementally throughout the tidal cycle, adjusting for channel width based on an estimated lateral profile derived from a nautical chart. As shown on Figure 4.5, salt flux was summed independently for each of the floods and ebbs through the cycle. The solid line in Figure 4.5 represents the net salinity flux associated with each salinity bin. The seaward and landward directed salt fluxes balance to within $2 \%$ through the entire tidal cycle, suggesting that this two-dimensional treatment of the observations may be representative of conditions across the channel at the anchor station. Significant lateral variability in flux would result in considerably poorer closure.

From the figure, it is apparent that salt mass enters the control volume at high salinities, but exits across a range of lower salinities, indicating that saline water is mixed with outgoing fresh water somewhere landward of the anchor station. This asymmetry in the distributions is strong evidence that mixing, as opposed to horizontal advection induced by tidal oscillations, is the dominant mechanism responsible for removal of salt from the channel during the ebb. 


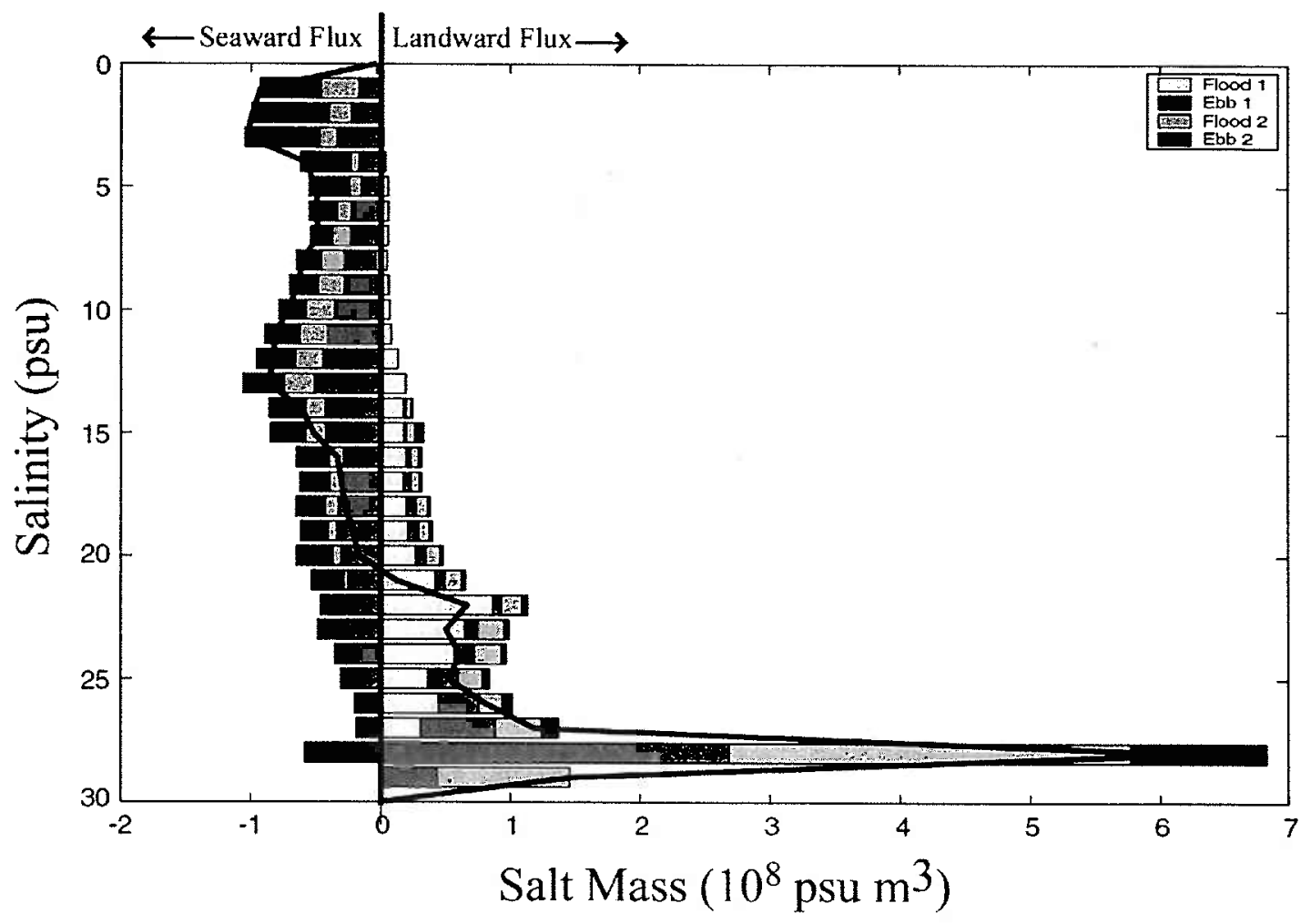

Figure 4.5: Bars represent total salt mass fluxed past the anchor station $(3.5 \mathrm{~km}$ landward of Sand Heads) during a complete tidal cycle, broken into tidal phases as shown. Positive and negative values represent landward-and seaward-directed fluxes, respectively. The solid overlying curve represents the net salt mass transport for each salinity bin. The salinity scale is inverted to approximate water column denth. 


\subsubsection{Buovancy Flux Estimates Using a Control Volume Approach}

A control volume approach for turbulence measurements was described in detail in Chapter 3. A similar approach is used here for two separate data sets: the integrated time series data discussed above, and a series of along channel control volumes defined by repeated observations at a series of fixed stations during the second ebb. In general, the method follows Equations (3.14) through (3.18), with some modifications.

In the case of the integrated time series data, mean buoyancy flux is estimated for the entire tidal cycle. The conceptual limits of the control volume for this analysis extend from the anchor station location to a point beyond the landward extent of the salt wedge excursion. The time series data adequately constrains the salt balance as all salt must enter and exit the control volume at the anchor station location. Extending the limits of the analysis across the entire period that salt is present also eliminates the time dependent term. Because the volume balance cannot be constrained by this data set, due to unknown freshwater velocities landward of the salt wedge, a vertical profile of entrainment velocity cannot be generated, as described in Chapter 3. Thus, it must be assumed that $\overline{S^{\prime} w^{\prime}}$ is equivalent to $\overline{S w}$, with the implication that the diahaline velocity, $w_{h}$, is zero. In fact, $w_{h}$ must be exactly zero at some level near the middle of the water column. This can be seen by combining equations (3.8) and (3.3),

$$
w_{h} \frac{\partial S}{\partial z}=\frac{\partial}{\partial z}\left(\overline{S^{\prime} w^{\prime}}\right)
$$

from which it follows that the diahaline velocity must be zero at the level of maximum

$\overline{S^{\prime} w^{\prime}}$. Furthermore, due to the change of sign of diahaline velocity through the water 
column, the assumption should also be reasonable with respect to averages taken in the vertical or across salinity space.

With these considerations, the salt equation for the time series control volume reduces to

$$
\overline{S^{\prime} w^{\prime}}(\tilde{S})=\frac{\int_{0}^{z_{\tilde{S}}} \int_{x y c l e} S_{\text {sub }} b d t d z}{\int_{\text {cycle }} A_{S I} d t}
$$

where $S, u, b$, and $z_{\widetilde{s}}$ are the mean salinity, mean along channel velocity, cross-channel width, and the observed depth of the given isohaline, respectively. The numerator of (4.11) represents the net gain or loss of salt mass above the chosen salinity threshold, $\tilde{S}$, which is represented in Figure 4.5 by the area under the solid curve. The projected area of a given isopycnal surface, represented as $A_{S I}$ in the denominator of (4.11), requires an estimate of the landward extent of the salinity intrusion beyond the anchor station. This was accomplished by taking the difference between running integrals of inbound and outbound salt flux to identify the mass of salt present in the channel as a function of time. Combining this information with an observation of the location of the head of the salt wedge during an earlier tidal cycle allowed the length of the salinity intrusion to be estimated. To generate a time series of isopycnal area, a representative channel width of $700 \mathrm{~m}$ was applied to the time series of salt wedge length.

Vertical turbulent salt flux, as evaluated in (4.11), can be converted to a buoyancy flux using (4.3). A profile of buoyancy flux relative to salinity for the time series data is presented in Figure 4.6. These values represent a temporal and spatial mean of the 


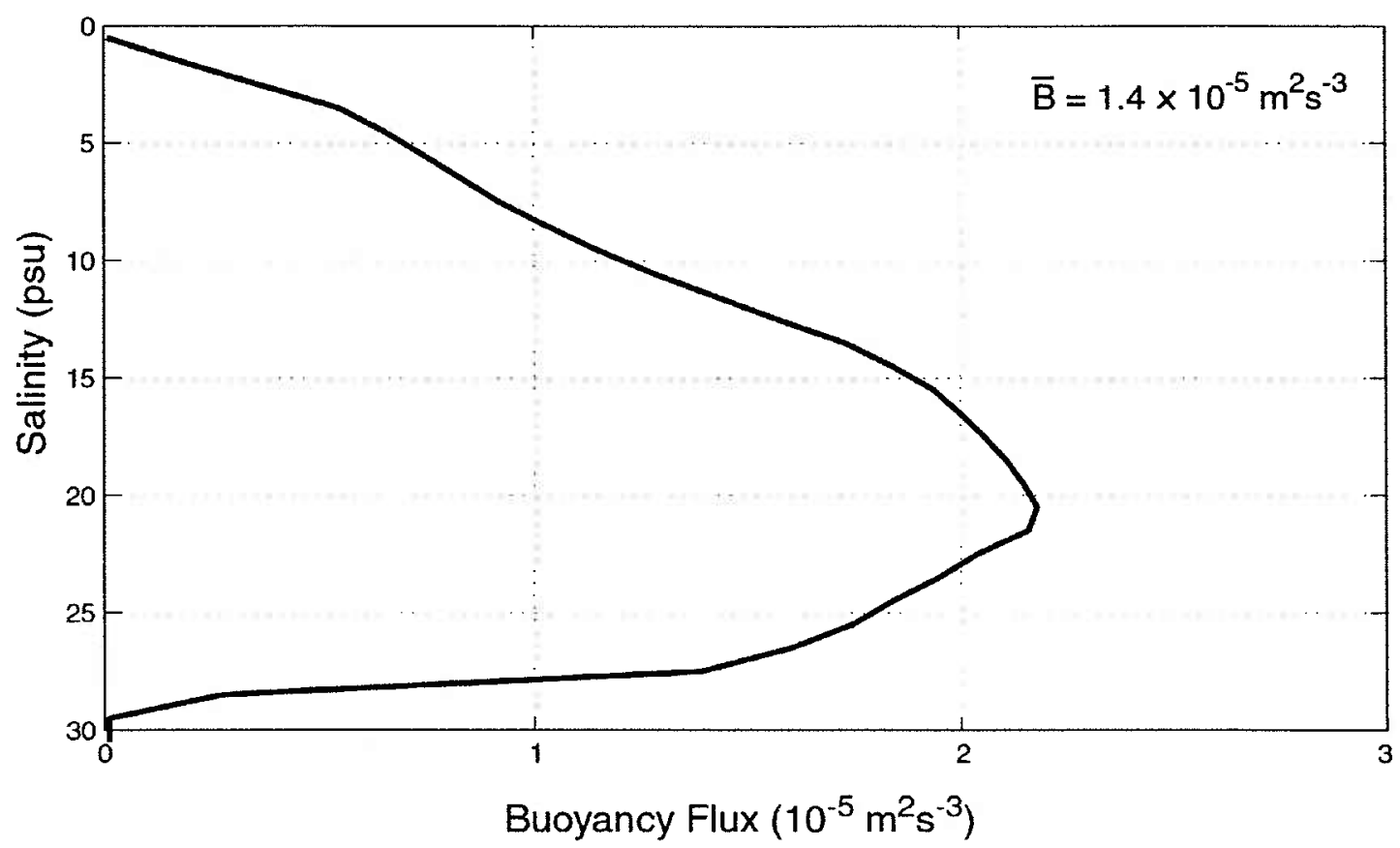

Figure 4.6: Profile of temporally and spatially averaged buoyancy flux with respect to salinity. The average buoyancy flux across salinity space is $1.4 \times 10^{-5} \mathrm{~m}^{2} \mathrm{~s}^{-3}$. 
buoyancy flux across the entire tidal cycle and the extent of the salinity intrusion.

Extending the mean across salinity space yields a value of $(1.3 \pm 0.1) \times 10^{-5} \mathrm{~m}^{2} \mathrm{~s}^{-3}$. Note that this mixing is approximately an order of magnitude less intense that the mixing observed seaward of the front at the end of the ebb, as discussed in Chapter 3. Assuming an average estimated buoyancy frequency of $N=0.12 \mathrm{~s}^{-1}$, and a reduced gravity of $g^{\prime}=0.15 \mathrm{~ms}^{-2}$, estimates of the mean turbulent eddy diffusivity and entrainment velocity scale through the tidal cycle landward of the anchor station are $K_{z}^{\rho}=9 \times 10^{-4} \mathrm{~m}^{2} \mathrm{~s}^{-1}$, and $w_{t}=9 \times 10^{-5} \mathrm{~ms}^{-1}$, respectively.

The control volume approach utilized in the upstream channel is more consistent with the method as described in Chapter 3. Data were collected during the second ebb from a series of cross-channel CTD "fences", typically consisting of three to four CTD stations coupled with ADCP velocity data. A circuit consisting of multiple fences was sampled repeatedly during the course of the observation period. Analysis of two adjacent fences (typically separated by a half to one kilometer) was conducted by summing fluxes in the cross channel direction assuming each station was representative of conditions across a specific width. This procedure resulted in the integration of any cross channel variability, allowing the volume and salt budgets to be constrained, and an estimate of the Reynolds salt flux to be generated following (3.17) as

$$
\overline{S^{\prime} w^{\prime}}=\overline{S w}-\bar{S} \bar{w}
$$

Using this approach, combined with equation (4.3), profiles of buoyancy flux across salinity space were generated for 15 locations in time and space. All measurements were 
taken during the largest daily ebb on July $1^{\text {st }}, 2^{\text {nd }}$, and $3^{\text {rd }}$, and were located between the anchor station discussed above and the entrance to Steveston Harbor, approximately 8.7 $\mathrm{km}$ landward of Sand Heads. At each location, estimates were generated for each isopycnal between 1 and $28 \mathrm{psu}$, with a resolution of $1 \mathrm{psu}$.

Unlike the steady state situation described in Chapter 3, the conditions in the upstream channel during the ebb are highly time-dependent. In an attempt to account for the non-synoptic nature of the measurements, data from different locations used for a specific calculation were linearly interpolated to the same point in time prior to evaluation of the salt and volume balance terms. Errors can enter this process as a result of the frequency at which stations were sampled, and also through the estimation of the time dependent term based solely on information at the limits of the control volume.

The magnitude of the total error for each control volume analysis was assessed by determining if salt was balanced within each section. Observed errors were distributed linearly in $z$ to close the salt balance, and force $\overline{S^{\prime} w^{\prime}}$ to zero at the channel bottom. In order to minimize the impact of these time-dependent errors, individual estimates of buoyancy flux were averaged across many realizations in time and/or space. Mean values of the control volume estimates of buoyancy flux during the ebb are shown in Figure 4.7. These values represent averages of the corrected profiles taken vertically across the entire depth, temporally across the limits of the data during the large daily ebb, and spatially within the limits of $1 \mathrm{~km}$ along-channel bins. The upper limits of error shown in Figure 4.7 represent standard error. The lower limits represent the mean of both the corrected and uncorrected profiles. A mean buoyancy flux value for all of the 


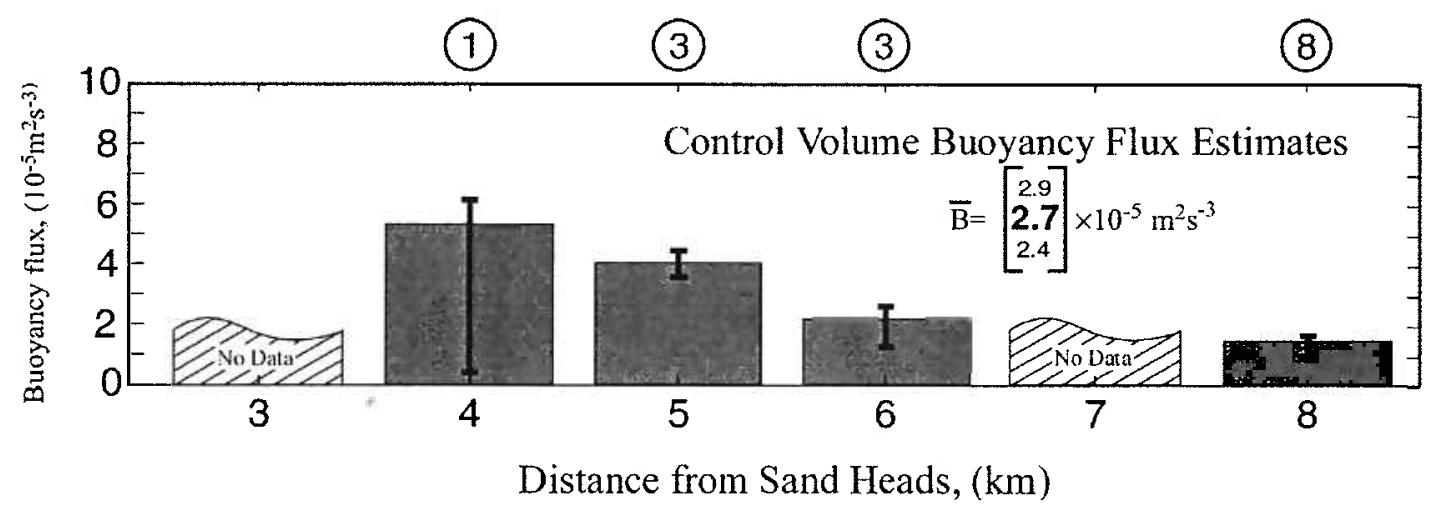

Figure 4.7: Spatial variability of buoyancy flux, $B$, as estimated from the control volume analysis, for observations made during the larger of the two daily ebbs (June 30 - July 4, 2000). All values represent the vertical and horizontal mean of all profiles generated within the $1 \mathrm{~km}$ long regions specified by the bars. The numbers in circles at the top of the panel indicate the number of profiles represented in each mean. Profiles were adjusted so that flux at the bottom was equal to zero. Errors were attributed mainly to the temporally evolving nature of the salt wedge during this period. The upper error bound represents standard error. The lower error bound represents the mean of both corrected and uncorrected profiles, as described in the text. 
control volume calculations during the ebb landward of the time series location, averaged across all dimensions (space, time, and salinity), is $2.7 \times 10^{-5} \mathrm{~m}^{2} \mathrm{~s}^{-3}$, with estimated error bounds as shown on Figure 4.7.

\subsubsection{Overturn Scale Estimates}

The overturn scale analysis utilized CTD data from the anchor station time series, as well as other casts performed between $3 \mathrm{~km}$ and $8 \mathrm{~km}$ from the mouth during the second ebb, including those utilized for the channel control volume calculations. Each individual CTD cast, resampled to $5 \mathrm{~cm}$ in the vertical, was sorted in order of increasing salinity, and compared with the original. A vertical offset was identified for each point as the smallest vertical displacement between an observed salinity, and the location of that salinity value within the sorted profile. The mean overturn scale for the cast was then taken as the rms value of all displacements (including 0, or non-displacements) within the cast. This method provided a natural scaling for stable regions of the water column, and proved a more effective method for identifying a mean cast overturn scale than averaging individual values of $L_{t}$ for each observed overturn (as described in Thorpe, 1977). The mean overturn scale for the cast was then combined with similar results from other casts to generate broader based estimates by averaging across time and space.

The top panel of Figure 4.8 shows the mean overturn scale observed through the tidal cycle at the anchor station, with temporal means taken across 2.67 -hour periods. The mean overturn scale through the entire tidal cycle is $25 \pm 2 \mathrm{~cm}$, with individual time period means ranging from 9 to $39 \mathrm{~cm}$. The bottom panel represents similarly averaged values of the overturn scale derived buoyancy flux, $B_{t}$, using (4.8). For these calculations, a 

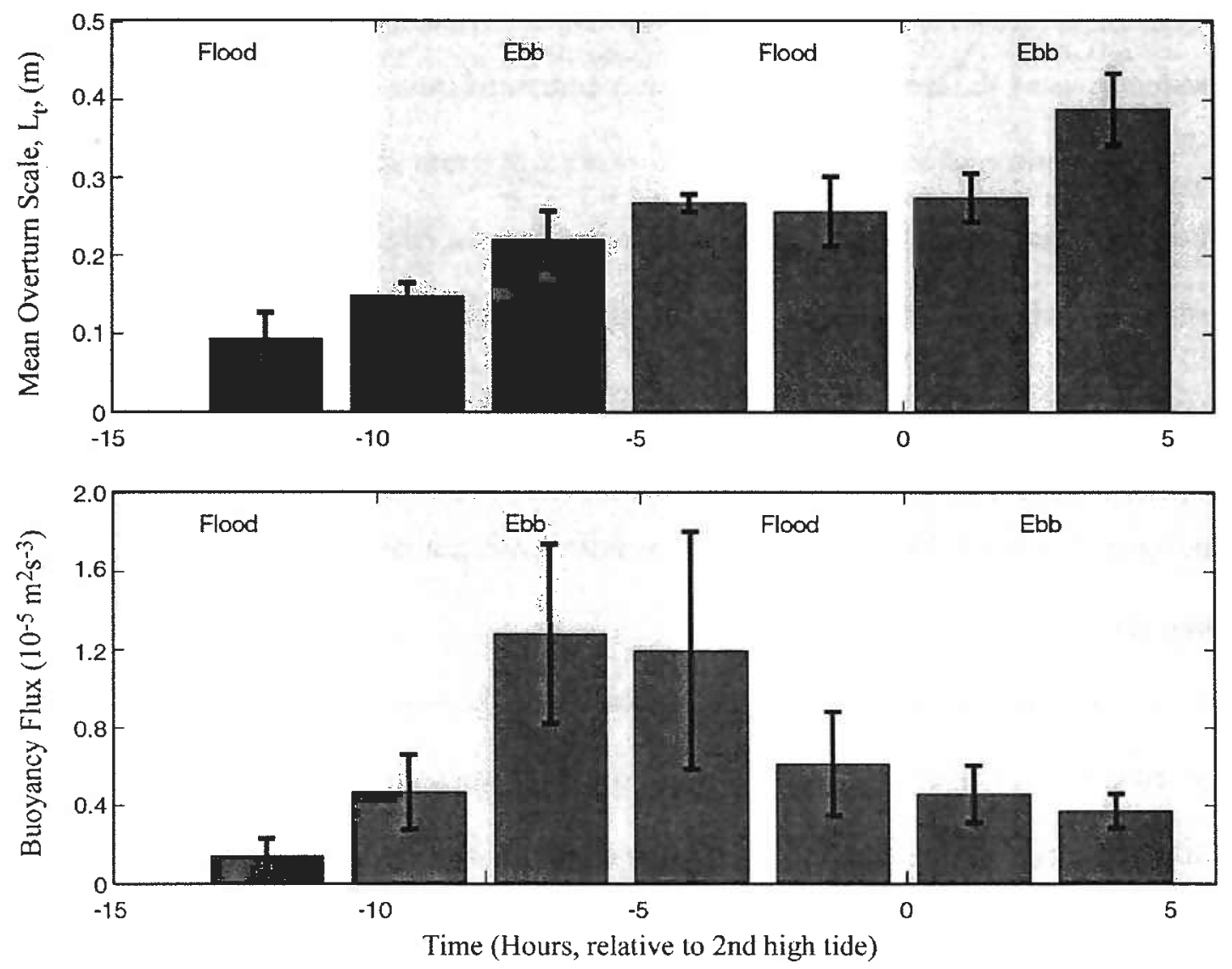

Figure 4.8: Temporal variability of the overturn scale, $L_{t}$, (top panel), and the associated buoyancy flux, derived using equation (4.8), through a tidal cycle at the time series location. Shaded regions represent ebb tides. 
buoyancy flux value was calculated for each individual displacement length, prior to averaging, using the local buoyancy frequency computed from the sorted profile.

A comparison of these results indicates that the overturn size steadily increases through the first flood and ebb, reaching a plateau near the beginning of the second flood, and then peaking sharply towards the end of the second ebb. However, estimates of buoyancy flux indicate that mixing is at a maximum during the later part of the first ebb, followed by a steady decline through the remainder of the tidal cycle. The decrease in buoyancy flux is not due to a decrease in overturn scale, but rather to a decrease in stratification.

This is illustrated in Figure 4.9, using a cast from the time series at the beginning of the second ebb as an example. The second panel compares the overturn displacement with buoyancy frequency, and shows that the majority of the displacements are happening within a well-mixed bottom water layer. In this case, a significant amount of "neutral" mixing (mixing of initially mixed water) is occurring, which is not operating against a significant density gradient, and therefore contributes little to the increase in potential energy described by the buoyancy flux. In contrast, the large overturn seen near $z=-3 \mathrm{~m}$ occurs within a region of high density gradient and is responsible for the majority of the mixing observed in the cast. The average buoyancy flux associated with the $Z=-3 \mathrm{~m}$ overturn is $1.4 \times 10^{-5} \mathrm{~m}^{2} \mathrm{~s}^{-3}$, with an average overturn scale of $12 \mathrm{~cm}$. For comparison, the average overturn scale across the entire cast is $23 \mathrm{~cm}$, with a corresponding mean buoyancy flux of only $8.0 \times 10^{-7} \mathrm{~m}^{2} \mathrm{~s}^{-3}$. 

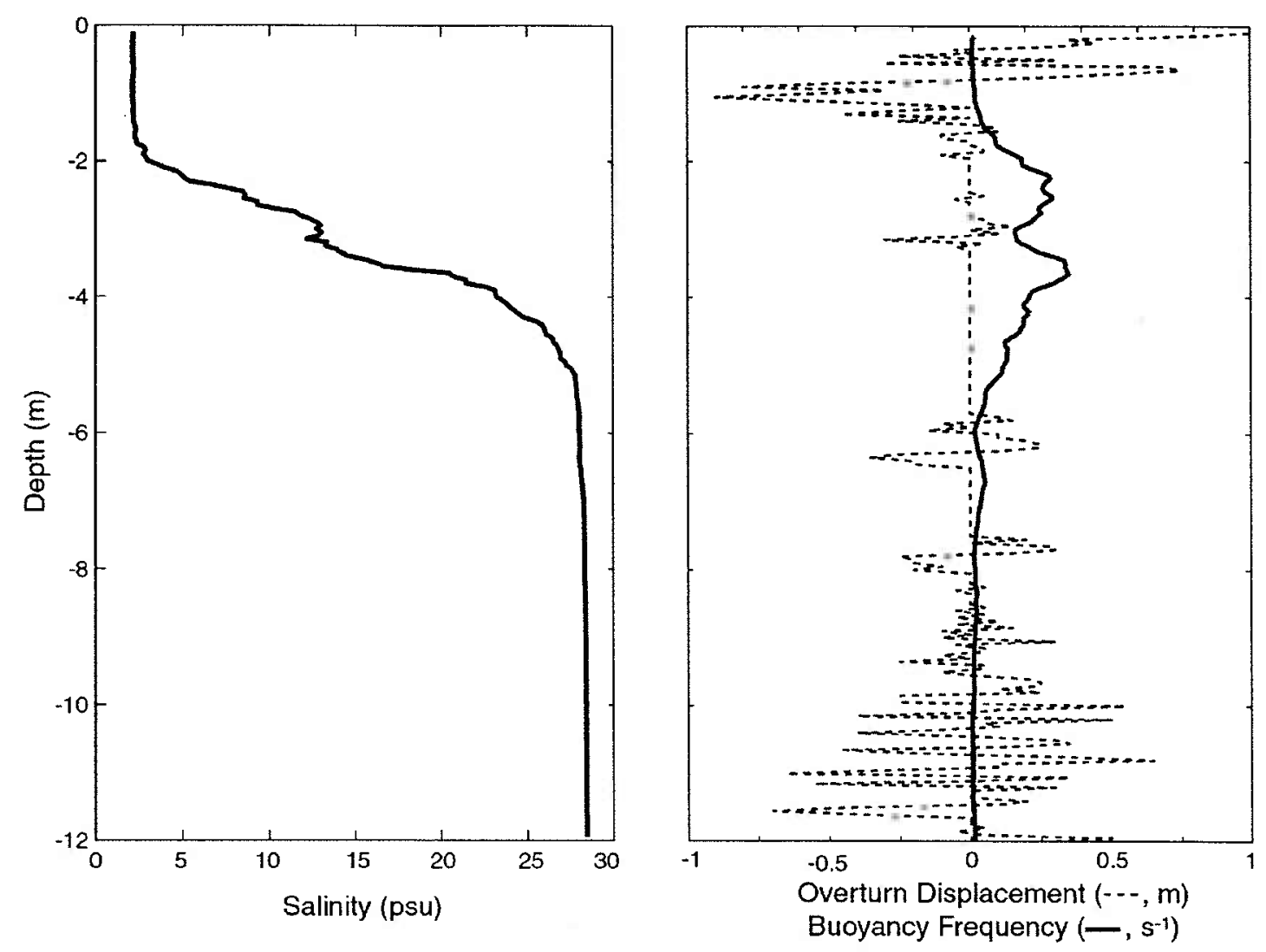

Figure 4.9: Salinity profile for CTD cast at the anchor station, two hours after second high tide. The second panel compares overturn displacement and buoyancy frequency. Mean buoyancy flux for the entire cast is equal to $8 \times 10^{-7} \mathrm{~m}^{2} \mathrm{~s}^{-3}$; the mean is equal to $3 \times 10^{-6} \mathrm{~m}^{2} \mathrm{~s}^{-3}$ across the entire pycnocline; and $1 \times 10^{-5} \mathrm{~m}^{2} \mathrm{~s}^{-3}$ for the isolated overturn located at approximately 3 meters depth. This cast is typical for the period, with large displacements occurring in regions of negligible density gradient. 
Spatial means were generated by grouping channel casts into along channel bins with a dimension of $1 \mathrm{~km}$, as shown in the top panel of Figure 4.10 . The overall mean value for the channel region between 3 and $8 \mathrm{~km}$ from the mouth during the ebb portion of the tidal cycle was $38 \pm 4 \mathrm{~cm}$. Local means of $B_{t}$ are shown in the second panel of Figure 4.10 , with a mean value of $(1.4 \pm 0.6) \times 10^{-5} \mathrm{~m}^{2} \mathrm{~s}^{-3}$. The estimates of $B_{t}$ in the second panel are compared to the estimates of B generated from the control volume analysis that were presented in Figure 4.7. The two methods provide consistent results, within the limits of the errors associated with each method, and indicate that enhanced mixing is occurring within the $5 \mathrm{~km}$ bin. This location is just seaward of the bend, within a channel constriction.

\subsubsection{Stratification Profiles}

The stratification parameter, $\psi$, provides an integrated assessment of the combined effect of mixing and straining mechanisms, which are both dependent on the shear $(\Delta u)$ as described above. Figure 4.11 shows the evolution of $\psi$ and $\Delta u$ through a tidal cycle as observed during the time series. Focusing initially on the stratification parameter, $\psi$, we see that the water column is stratified throughout the tidal cycle, with stratification increasing dramatically to highly stratified conditions near the middle of each ebb tide, and conditions approaching a more partially mixed situation during floods. Comparison with the velocity difference indicates that high shears during the later portions of the ebbs may be responsible for limiting the increase in stratification that occurs during the early portions of both ebbs. Stratification corresponding to the spatial profiles of the second 

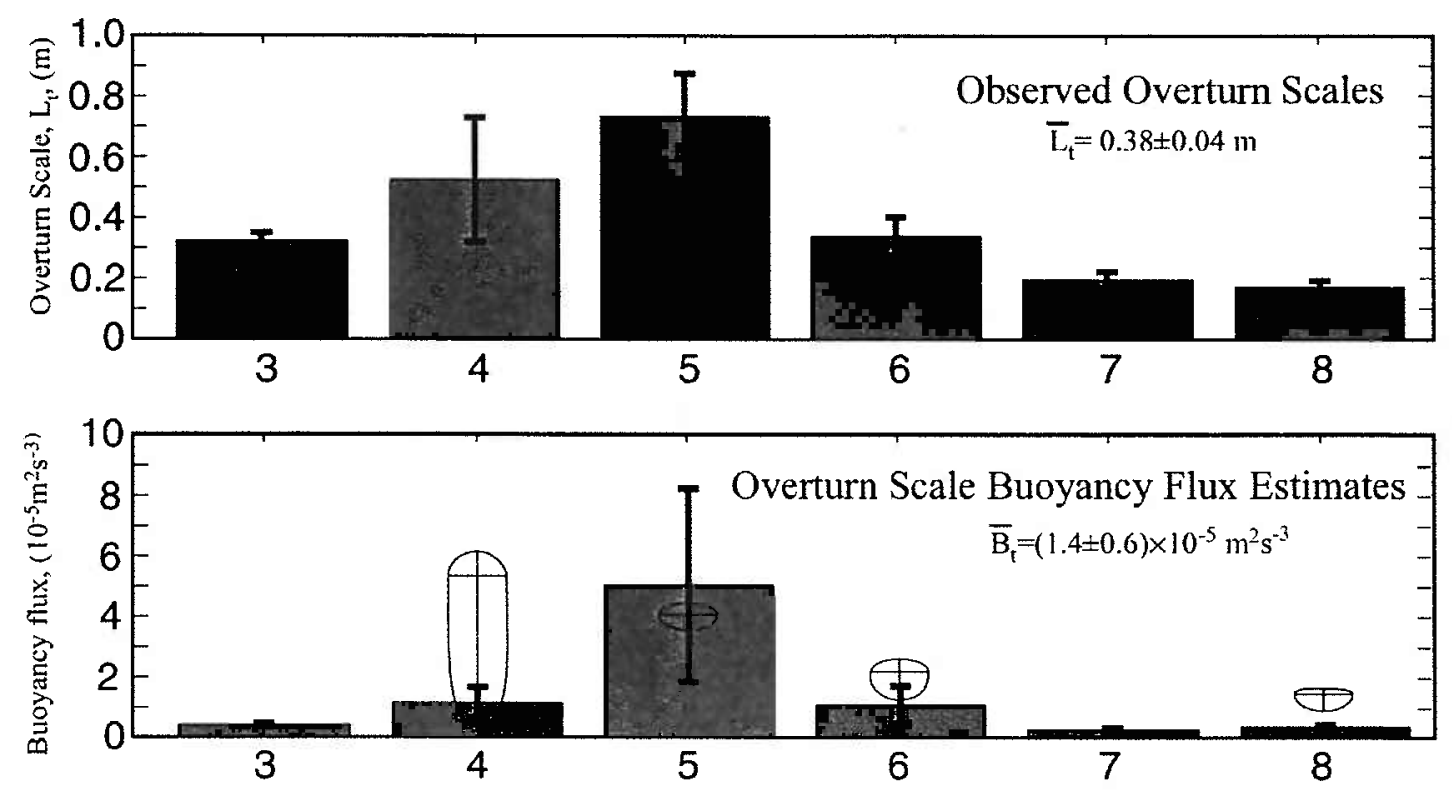

Figure 4.10: Spatial variability of the observed overturn scale (top panel), and buoyancy flux, $B_{t}$, as estimated from the overturn. All values represent the mean of observations from the larger of the two daily ebbs (June 30 - July 4, 2000). The error bars shown represent standard error. Crosses in the bottom panel represent the mean and error limits from the control volume method (Figure 4.7) for comparison. 


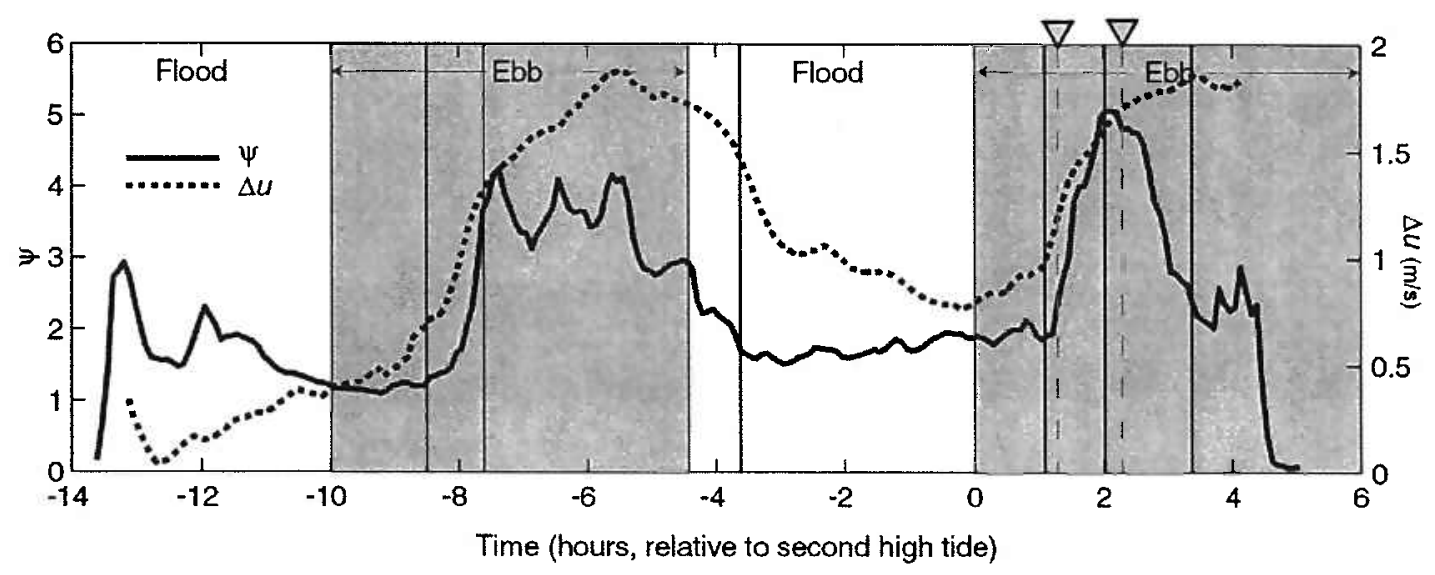

Figure 4.11: Stratification parameter, $\psi$, and velocity difference between upper and lower layer, $\Delta u$, at time series location, approximately $3.4 \mathrm{~km}$ landward of Sand Heads. Shaded regions represent ebb tides. Vertical lines are for reference in comparison with other figures. Triangles at top of figure identify the times of the two ebb profiles shown in Figure 4.2. 
ebb shown in Figure 4.2 are not plotted, but values of $\psi$ are consistently between 2 and 3 for both profiles, indicating a uniform highly stratified condition.

\subsubsection{Richardson Number Profiles}

The gradient Richardson number was evaluated for both the time series data and the two salinity profiles from the ebb portion of the tidal cycle presented in Figure 4.2. As discussed above, a value of $R i_{g}$ at or below $1 / 4$ is considered a necessary condition for the onset of turbulence. However, turbulence in regions of weak stratification results in "neutral" mixing, where the weak gradients limit effective contributions to the buoyancy flux. In order to focus on regions that can result in a positive buoyancy flux an additional criteria of $d S / d z>0.3 \mathrm{psu} \cdot \mathrm{m}^{-1}$ was applied to the gradient Richardson number results. Figure 4.12 shows a profile of $R i_{g}(\%)$ for the time series. This parameter represents the percentage of the water column that is both subcritical with respect to the local gradient Richardson number and meets the salinity gradient criteria.

An alternative to this approach is to plot the average gradient Richardson number across the pycnocline. This value, known as a bulk Richardson number, was calculated using the $L_{50}$ length scale of the pycnocline as introduced above:

$$
R i_{B}=\frac{g \frac{\Delta \rho}{\rho} L_{50}}{(\Delta u)^{2}}
$$

where $\Delta$ represents a difference across the limits of the pycnocline defined by $L_{50}$. The value of $R i_{B}$ through the time series is plotted in Figure 4.13.

The $R i_{g}(\%)$ distribution for the time series data, shown in Figure 4.12, shows an increase in mixing propensity during both ebbs, with water column percentages of 25 to 


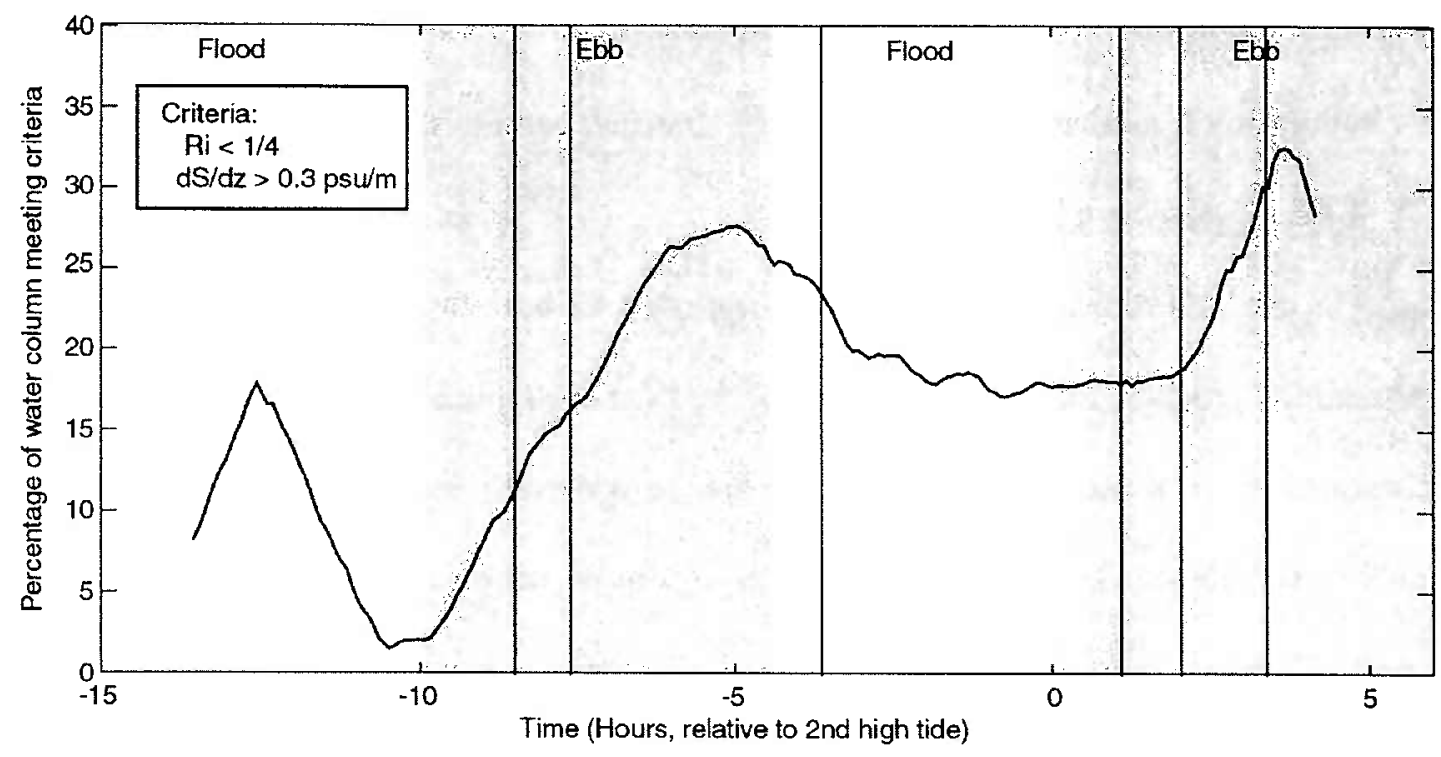

Figure 4.12: Percent of water column with Richardson number $\left(R i_{g}\right)$ less than $1 / 4$, and $\partial S / \partial z$ greater than a minimum threshold value of $0.3 \mathrm{psu} / \mathrm{m}$ for the time series data through one complete tidal cycle (July 3-4, 2000). Shaded areas represent ebb tides. Vertical lines are for reference in comparison with other figures. 
$30 \%$ meeting the threshold criteria. Note that the interfacial region, where the greatest buoyancy flux is likely to occur, rarely spans more than a third of the water column, as indicated by the stratification parameter in Figure 4.11. Thus, values of 15 to 30 percent and greater are significant, as they indicate that conditions are conducive to turbulent mixing across the majority of this region. An additional, smaller peak is observed at the beginning of the time series, indicating a tendency for lower Richardson numbers associated with the head of the salt wedge. This peak is immediately followed by a period of high Richardson numbers as the percentage of the water column satisfying the $R i_{g}$ and $d S / d z$ criteria drops to less than $3 \%$. Little relaxation of the curve is seen during the second flood, where water column percentages of 15 to $20 \%$ are observed.

The $R i_{B}$ profile plotted in Figure 4.13 is generally consistent with the profile in Figure 4.12 , with the notable exception of the later portions of the second ebb, where $R i_{B}$ values are greater than $1 / 4$, but a high percentage of the water column is observed with subcritical Richardson numbers. This suggests that the region of active mixing has been depressed beneath the region identified by the $L_{50}$ scale (approximately 7 psu to $21 \mathrm{psu}$ ).

The spatial variability of the Richardson number during the ebb is shown by plotting $R i_{g}(\%)$, similar to the plot in Figure 4.12. Both ebb profiles (2.3 and 3.3 hours) are shown in one panel on Figure 4.14. A larger portion of the water column shows a propensity for mixing near the salt wedge head $(x \approx 10 \mathrm{~km})$, and seaward of about $x \approx 5, \mathrm{~km}$, than in the intermediate region. Both of the profiles in Figure 4.14 are similar to the buoyancy flux estimates, in that lower mixing activity is observed within the bend 

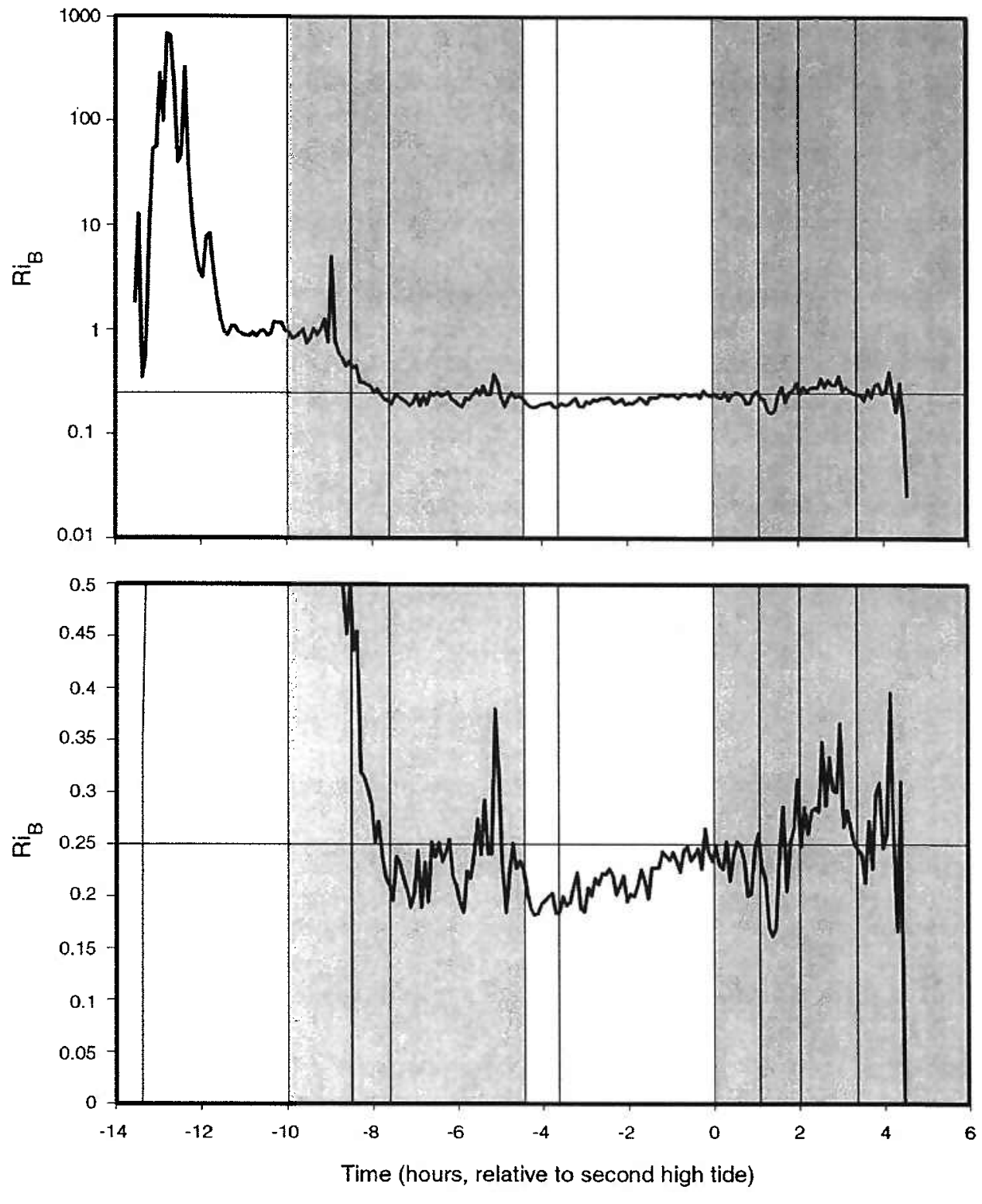

Figure 4.13: Bulk Richardson number across the pycnocline defined by $L_{50}$ (approximately 7 psu to $21 \mathrm{psu}$ ). Top panel is plotted on a log scale, and bottom on a linear scale to provide increased resolution near the value of $1 / 4$. In both panels, $R i_{B}=1 / 4$ is indicated by the horizontal line. Vertical lines are provided for reference in comparison with other figures. 


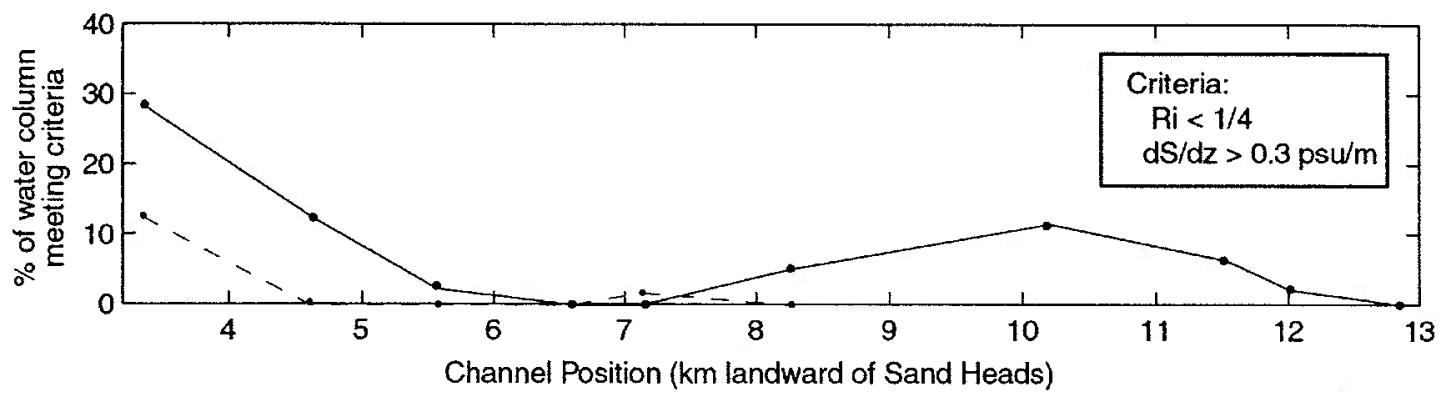

Figure 4.14: Percent of water column with Richardson number (Ri) less than $1 / 4$ and $\partial S / \partial z$ greater than $0.3 \mathrm{psu} / \mathrm{m}$, for ebb profiles at 2.3 hours (dashed line) and 3.3 hours (solid line) after second high tide. Note that the profile at 2.3 hours does not extend to the head of the salt wedge. Richardson number profiles were calculated at the cast locations indicated on Figure 4.2. 
$(x \approx 6,000 \mathrm{~m})$, than seaward of the bend. Values of $R i_{B}$ are not shown, but are consistent with the plot in Figure 4.14.

\subsubsection{Froude Number Profiles}

Profiles of the individual layer Froude numbers and composite Froude number through the time series are shown in Figure 4.15. The flow is seen to be significantly supercritical during both ebbs, and subcritical during floods. It is interesting to note that the lower layer is observed to be the active layer until several hours into the first ebb, at which point the lower layer Froude number drops nearly to zero. Although these values increase during the second flood, the upper layer values never decrease sufficiently to allow the lower layer to again dominate the dynamics of the two-layer system.

The composite Froude numbers for both channel profiles are shown in Figure 4.16. In both profiles the Froude number expresses a tendency towards critical values $(G=1)$ across the majority of the region, which may indicate a feedback mechanism between accelerating upper layer velocities and mixing. A distinct increase towards significantly supercritical values is observed in the vicinity of the narrows at $5 \mathrm{~km}$, however, consistent with the supercritical values $(G \sim 2)$ observed during the ebb at the anchor station, as shown in Figure 4.15. These supercritical Froude numbers are likely reflective of a local acceleration due to the channel constriction, an acceleration that is substantially greater than in other regions of the channel. Although mixing is enhanced due to this acceleration, the length scales required to return the Froude number to a critical value of unity appear to be on the order of several kilometers, at least, and a significant region of supercritical Froude numbers persists in the channel during this period. In this manner, 


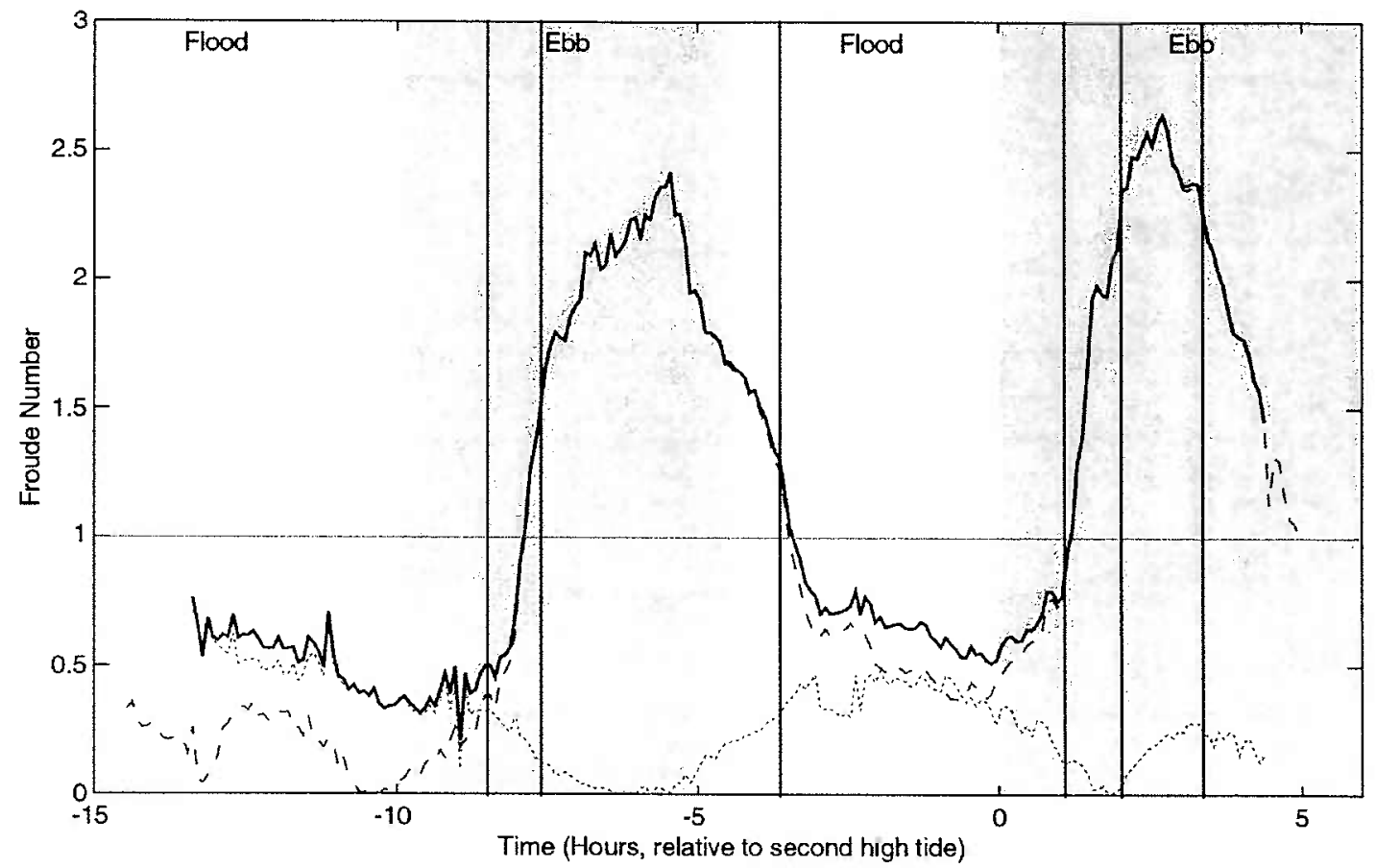

Figure 4.15: Internal composite Froude number (solid line), and individual layer Froude numbers (upper layer- dashed; lower layer- dotted) for the time series through one complete tidal cycle (July 3-4) at the anchor station located $3.4 \mathrm{~km}$ landward of Sand Heads. Shaded areas represent ebb tides. Vertical lines are provided for reference in comparison with other figures. 


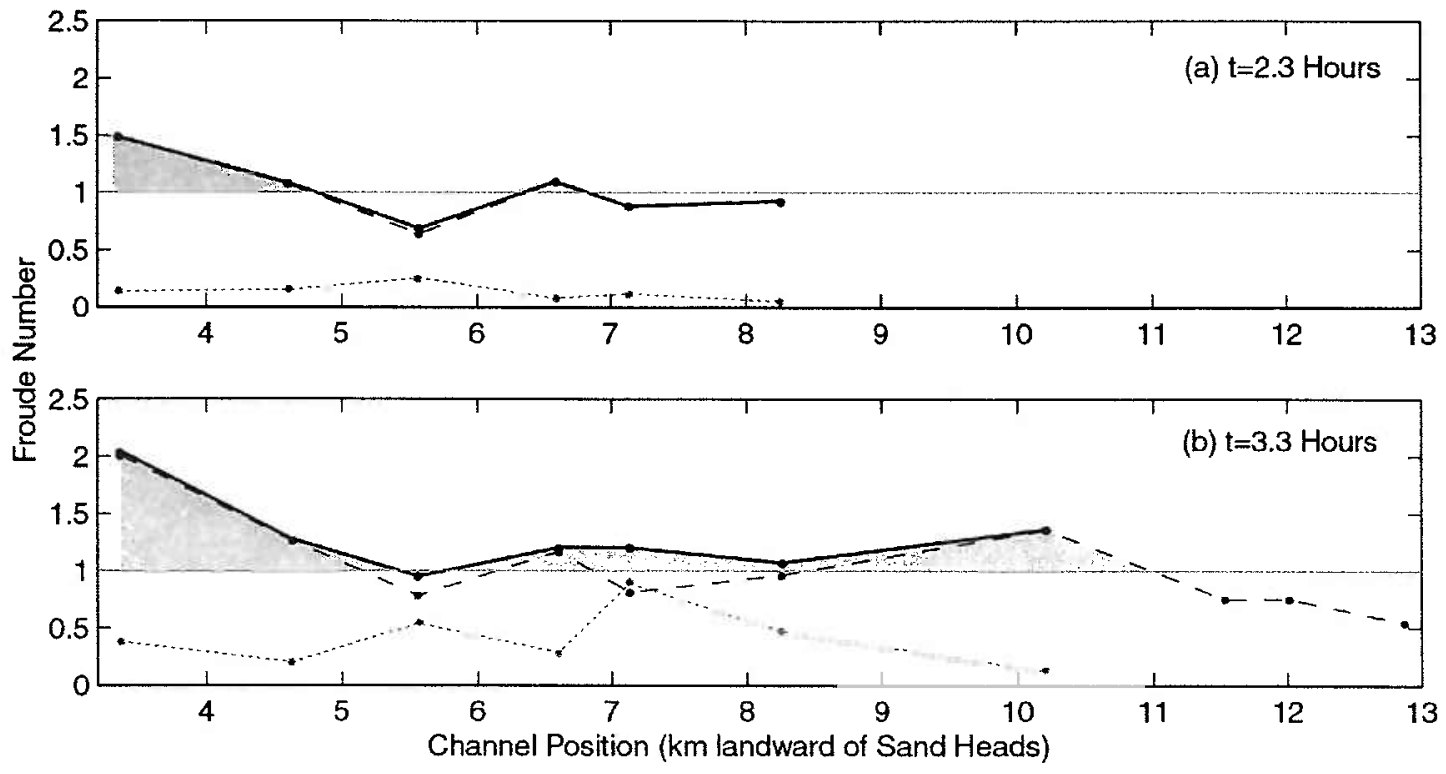

Figure 4.16: Internal composite Froude number (solid line), and individual layer Froude numbers (upper layer- dashed; lower layer- dotted) for the ebb profiles shown in Figure 4.2. Froude number values were calculated at the cast locations indicated on Figure 4.2. 
regions of significantly supercritical Froude numbers $(G>2)$ may be an effective indicator of enhanced mixing processes.

\subsection{Temporal Variability of Mixing Processes}

In the following subsections, the temporal evolution of the salt wedge structure is discussed with respect to the four main periods of the tidal cycle, by referring to the time series data presented in Section 4.4 .

\subsubsection{Initial Flood}

During the initial flood, mixing rates are low, as evidenced by the plots of $B_{t}$ (Figure 4.8), $R i_{g}(\%)\left(\right.$ Figure 4.12), and $R i_{B}$ (Figure 4.13). Stratification, as shown in Figure 4.11, is nearly constant during this period at values that are low relative to the range of values observed through the tidal cycle, indicating the presence of a significant quantity of mixed water. This mixed water is not the result of local mixing processes, but delivered landward through advective processes, as shown by the temperature-salinity (T-S) diagram in Figure 4.17. This figure shows the T-S curves for each of the CTD casts performed at the anchor station in the upper panel. The lower panel indicates the mean deviation of the water column from a conservative mixing line connecting the two endpoints of fresh, warm, river water $\left(0 \mathrm{psu}, 15^{\circ} \mathrm{C}\right)$ with cold, saline, Georgia Strait Water $\left(28 \mathrm{psu}, 11.5^{\circ} \mathrm{C}\right)$. It is evident from this figure that a third water mass is contributing to mixed water observed on the initial flood, most likely warm, brackish water from the shallow regions adjacent to Sand Heads, that moves in on the advancing tide. 

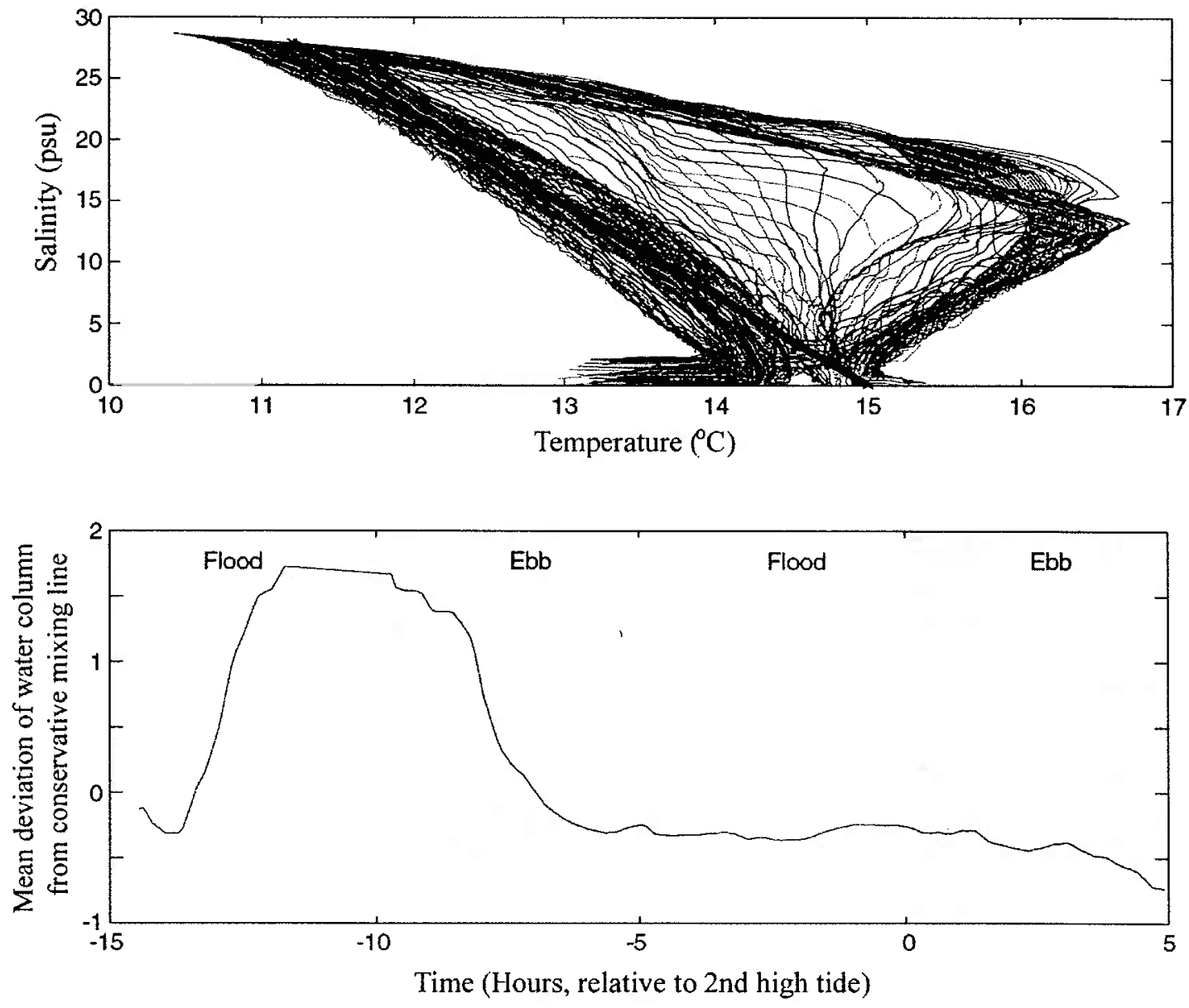

Figure 4.17: Temperature salinity relationships for time series CTD casts. The upper panel represents a composite plot of all CTD casts. A conservative mixing line, connecting cold, saline Georgia Strait water with warmer, fresh river water is shown in bold. In the bottom panel, the mean deviation of each cast from the conservative mixing line in the upper panel is plotted. 


\subsubsection{First Ebb}

The interaction of dynamic processes during the first ebb is best considered by dividing the ebb into three periods, as indicated by the vertical lines on the plots of time series data. The first hour and a half has conditions similar to the initial flood, with relatively constant stratification and low surface velocities. The plot of $R i_{g}(\%)$ indicates that isolated bursts of mixing may be occurring, despite generally large values of $R i_{B}$, which contribute to the slight increase in $B_{t}$.

During the next hour the surface velocity begins to accelerate rapidly, driving the flow towards supercritical, and initiating an estuary-wide straining mechanism that serves to flush mixed water seaward within the upper layer, and increase the local stratification. During this period, mixing continues to be suppressed, as indicated by $R i_{B}$ values that are maintained above $1 / 4$. As the value of $R i_{B}$ drops below $1 / 4$, mixing expands across the pycnocline, stemming the increase in stratification. The nearly constant value of the stratification parameter for the next two hours suggests equilibrium between the rates of mixing and straining.

\subsubsection{Second Flood}

The last hour of the ebb, and first hour of the second flood are characterized by a decrease in stratification, representing an adjustment due to significant changes in $\Delta u$, similar to that observed during the first ebb. In this case, straining begins to decrease with $\Delta u$, but intense mixing is maintained until the pycnocline begins to broaden. Eventually, a new equilibrium is established at stratification levels similar to the initial flood. Mixing continues through the flood, driven by shears that never fall below $1 \mathrm{~m} \cdot \mathrm{s}^{-1}$, 
maintaining subcritical bulk Richardson numbers. Mixing decreases, however, due to weakening gradients within the pycnocline, as shown by the $B_{t}$ estimates. Although the salinity structure during the second flood looks qualitatively similar to the first, as seen in Figure 4.4, the structure observed during the second flood cannot be attributed to the landward advection of mixed water because velocities within the region are primarily directed seaward as shown by the dashed line in Figure 4.4. The mixed water must be produced by mixing occurring at or landward of the anchor station.

\subsubsection{Final Ebb}

Between hours 1 and 2 of the final ebb, a third adjustment period is observed, similar to that observed during the first ebb. During this period, a subcritical spike occurs in the $R i_{B}$ profile, indicating a brief period of potentially intense mixing, but by the end of this period $R i_{B}$ is greater than $1 / 4$. The profile of $R i_{g}(\%)$ indicates an opposing trend, as it continues to increase for several hours. This suggests a depression of the active turbulent region below the $L_{50}$ pycnocline, consistent with the shaded region in Figure 4.4, and a corresponding drop in buoyancy flux due to relatively weak gradients. The majority of the mixed water during the later portion of the ebb is advected seaward without shears large enough to initiate significant mixing. This advection dramatically reduces the local stratification.

Approximately 1.5 to 2 hours prior to the removal of salt from the channel, the nearbottom velocities reverse, as shown in Figures 4.3 and 4.4, allowing the seaward advection of deep water for the first time since the beginning of the tidal cycle. These velocities can only account for approximately $1 \mathrm{~km}$, or less than $10 \%$, of the total wedge 
length, suggesting that the vast majority of the wedge is removed through a vertical collapse, consistent with conclusions derived from Figure 4.5.

\subsection{Spatial Variability of Mixing Processes During Ebb Tide}

The control volume and overturn scale estimates of buoyancy flux in the upstream channel during the ebb are compared in Figure 4.10. In most cases, the error bounds from the two estimates overlap, and both along channel profiles suggest a region of enhanced mixing associated with the channel constriction approximately $5 \mathrm{~km}$ landward of Sand Heads. This region is also characterized by an increase of Froude numbers to supercritical values from values that are otherwise nearly critical (Figure 4.16), and an increase in $R i_{g}(\%)$.

Geyer (1985, Figure 47) suggested that mixing during ebbs in the Fraser channel is focused near constrictions, providing visual evidence from echosounder output for four significant constrictions in the lower $20 \mathrm{~km}$ of the estuary. The data presented here provide quantitative support for these observations. The ebb profiles investigated here are composites from several days of operations in the channel, and represent conditions at 2.3 and 3.3 hours after high tide. The time of these observations is indicated on the stratification diagram in Figure 4.11, where it can be seen that the first profile coincides with the beginning of the increase in stratification, and the second with the peak in stratification. Both of these occur during periods when mixing is relatively suppressed, and no data is available to evaluate Froude numbers and Richardson numbers during the highly active mixing period that results in the collapse of the salt wedge. 
Another potential mixing mechanism worth consideration is the possibility of secondary circulation processes associated with the large bend in the channel at $6 \mathrm{~km}$. The establishment of strong secondary circulation in the bend could force deep water southward, and up onto the shallow areas at the inside of the bend, where enhanced shears due to bottom friction could provide an efficient mechanism for mixing, as shown in Figure 4.18. Due to the dangers of maneuvering the research vessel in shallow water, this region was not sampled during the field study, so it is possible that the overall estimates of buoyancy flux during the ebb in the upstream channel may be underestimated.

\subsection{A Summary of Mixing Through the Tidal Cycle}

\subsubsection{Mixing in the Fraser}

The structure of the salt wedge in the Fraser River is initially established through advective processes, and subsequently modified through an equilibrium between mixing and straining mechanisms. Each phase of the tide is characterized by an adjustment period on the order of 1 to 3 hours, where changes in shear trigger a change in the equilibrium between mixing and straining. Increases in shear move the system toward more highly stratified conditions, while stratification is relaxed during periods of lesser shear.

Mixing is active through the entire tidal cycle, with the exception of the very initial stages of the first flood, but enhanced during ebbs. An estimate of average mixing during the later portions of the first ebb (hours -7.9 to -4.5 ) was accomplished using a method 


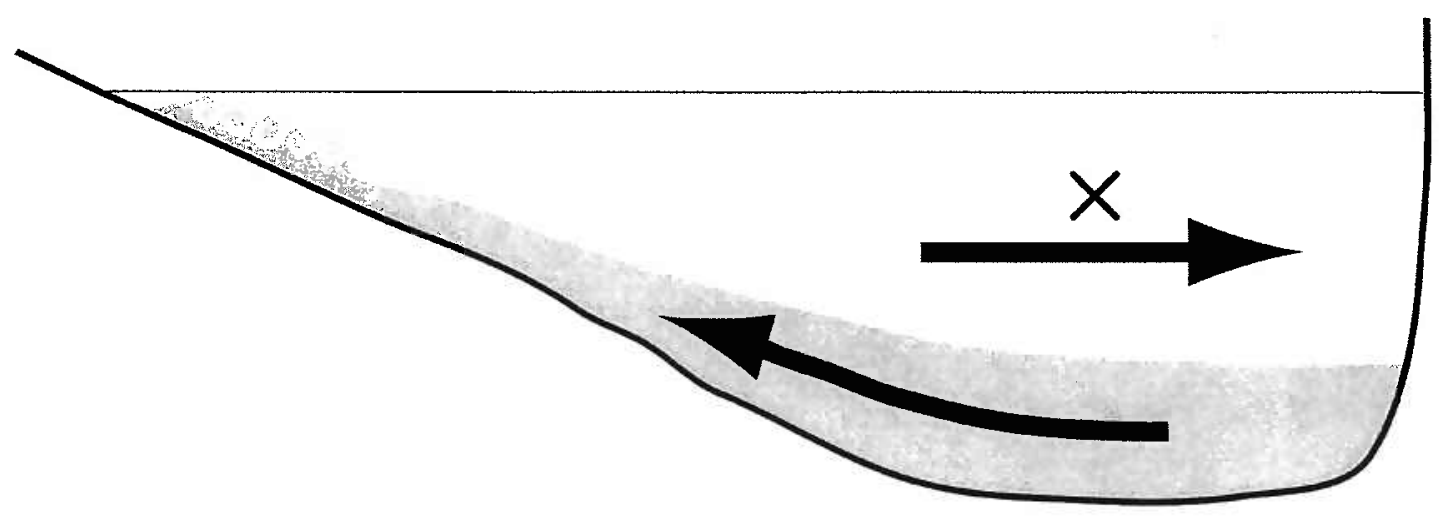

Figure 4.18: Schematic representation of the enhancement of boundary mixing due to secondary circulation. In this figure, the primary component of the fresh water velocity is directed into the page, and the channel is bending to the left. The induced cross-channel circulation pattern that develops is responsible for driving dense fluid up into the shallow regions of the channel located on the inside of the bend, where mixing is more intense due to the increased importance of bottom friction. 
similar to the modified control volume method employed for the entire tidal cycle in Section 4.4. In this case, the observed outflux during the period was compared to the total influx distribution observed through -4.5 hours. Using Equations (4.11) and (4.3), and the estimated salt wedge length described in Section 4.2, an estimated profile of buoyancy flux with a mean of $1.6 \times 10^{-5} \mathrm{~m}^{2} \mathrm{~s}^{-3}$, and a peak of $3 \times 10^{-5} \mathrm{~m}^{2} \mathrm{~s}^{-3}$ was obtained. These values represent a conservative estimate because it was assumed that no water mixed during the period remained upstream of the anchor station.

Observations by Geyer (1985) suggest that the salt wedge is uniform and highly stratified prior to the final mixing events that result in the ultimate collapse of the wedge. This point in time is coincident with the peak in stratification observed near hour +2 , shown on Figure 4.11, and is consistent with the salt wedge salinity profiles shown in Figure 4.2. An estimated buoyancy flux profile for the second ebb was generated using an output distribution equal to the total outflux of salt observed at the anchor station after +2 hours. The input distribution required for Equation (4.11) was estimated using the total mass of seaward directed salt after +2 hours, and a hypothetical vertical salinity profile based on the longitudinal profiles of Figure 4.2 and Geyer (1985, Figure 9). This exercise suggested a peak buoyancy flux during the final collapse of the salt wedge equal to $5 \times 10^{-5} \mathrm{~m}^{2} \mathrm{~s}^{-3}$, with a mean value of approximately $3 \times 10^{-5} \mathrm{~m}^{2} \mathrm{~s}^{-3}$. These values are slightly larger than, but generally consistent with, the average buoyancy flux values observed in the upstream channel during the ebb, that were presented in Figure 4.7.

The vast majority of the salt wedge is removed from the channel through a rapid and energetic collapse of the wedge during the second ebb. High shears, associated with the 
ebbing discharge, and increased within localized channel constrictions lead to this collapse, as suggested by Geyer (1985). This conclusion has been supported by observations of buoyancy flux within the channel during this study, as well as indications that Froude numbers become significantly supercritical within the narrows. It is likely that a similar collapse may occur during the first ebb, but the barotropic forcing mechanisms associated with the weaker ebb are insufficient to flush the remaining salt from the channel.

Given the overall estimate of mean buoyancy flux associated with the salt wedge that was calculated in Section 4.4, the estimates for the two ebbs presented above, and the overturn and control volume estimates of buoyancy flux during the ebb presented in Figures 4.7 and 4.10, a mean buoyancy flux value for the remaining portions of the tidal cycle (both floods in addition to the first few hours of each ebb) can be estimated. Table 4.1 summarizes the previous estimates, as well as the length of time for which each estimate is considered representative. The last column in table 4.1 is the product of this time and a representative salt wedge length. As shown on line 6 of Table 4.1, a mean buoyancy flux for the ebb portions of the tidal cycle is approximately $(2.2 \pm 0.8) \times 10^{-5}$ $\mathrm{m}^{2} \mathrm{~s}^{-3}$, representing approximately 6.6 hours of the tidal cycle. A representative buoyancy flux value for the remaining portions of the tidal cycle can then be estimated using the mean ebb estimate from line 6 and the tidal cycle average shown on line 1 , yielding a buoyancy flux value of $(1.0 \pm 0.5) \times 10^{-5} \mathrm{~m}^{2} \mathrm{~s}^{-3}$.

It is likely that there is still considerable variability within the remaining 11.8 hours of the tidal cycle. For example, lower rates of buoyancy flux may be associated with the 


\begin{tabular}{|c|c|c|c|}
\hline Description of Estimate & $\begin{array}{c}\text { Mean B } \\
\left(\times 10^{-5} \mathrm{~m}^{2} \mathrm{~s}^{-3}\right)\end{array}$ & Hours & $\begin{array}{c}\int L d t^{2} \\
(\times \mathbf{1 0} \mathbf{m} \cdot \mathbf{h o u r})\end{array}$ \\
\hline 1Anchor Station Tidal Cycle Average & 14 & 184 & 170 \\
\hline 2Anchor Station First Ebb Estimate & 1.6 & 3.5 & 3.7 \\
\hline 3 Anchor Station Final Ebb Estimate & 3.1 & 3.1 & $\overline{2.2}$ \\
\hline & & & \\
\hline 4) Overturn Ebb Estimate & 1.4 & 7 & - \\
\hline 5. Control Volume Final Ebb Estimate & 2.7 & 3.3 & - \\
\hline 6 Mean Ebb Estimate ${ }^{\mathrm{b}}$ & $2.2 \pm 0.8$ & 6.6 & 5.9 \\
\hline & & & \\
\hline 7 Remainder Estimate (Mean) & $1.0 \pm 0.5$ & 11.8 & 11.1 \\
\hline
\end{tabular}

Notes: a. Integral represents product of wedge length, $L$, and time.

b. Mean B is average of lines 2 through 5 . Remaining columns are sum of lines 2 and 3 ,

Table 4.1: Summary of buoyancy flux estimates through the tidal cycle. Lines 1,2, and 3 are derived from the integrated anchor series flux calculations. Lines 4 and 5 correspond to the overturn and control volume analyses shown in Figures 4.7 and 4.10. The mean ebb estimate in line 6 represents the average of lines 2 through 5. The estimate for the remainder of the tidal cycle, in line 7 , is derived from lines 1 and 6 so that a weighted mean of lines 6 and 7 is equal to the mean buoyancy flux estimate on line 1 .

initial flood, as compared to the second flood, based on the observations of $R i_{B}$ and $R i_{g}$ at the anchor station. Nevertheless, this estimate is generally consistent with the temporal evolution of mixing presented in Section 4.5, where estimates of $B_{t}$ through the tidal cycle indicate a similar difference between flood and ebb, on the order of a factor of two. Buoyancy flux rates observed at the anchor station location are generally lower than the averages identified upstream, which is consistent with the idea that the majority of buoyancy flux occurs at local constrictions, but the ratio between ebb and flood intensities at the anchor station may be indicative of conditions throughout the channel. 
This analysis indicates that mixing processes associated with the salt wedge are important through all phases of the tide. Although differences between buoyancy flux rates on flood and ebb may be significant, and as high as a factor of six, the mixing that occurs during floods cannot be considered negligible.

\subsubsection{Comparisons With Other Estuaries}

Many studies of estuarine dynamics have focused on partially mixed estuaries, such as the Hudson River. Although many aspects of estuarine dynamics are applicable across the wide physical spectrum of estuaries, differences in stratification can result in differences in the magnitude and timing of mixing within an estuary. In general the highest buoyancy fluxes in the Fraser channel were seen during ebbs, both locally and at critical mixing locations within the channel (e.g., channel constrictions). This is consistent with previous analyses of the temporal variation of mixing in estuaries, both in highly stratified (Partch and Smith, 1978), and partially mixed regimes (Nepf and Geyer, 1996). Previous work in the Fraser (Geyer and Farmer, 1989) also suggests that the ebb dominates contributions to the buoyancy flux, and that the most productive mixing occurred as a response to lateral constrictions in the channel.

Numerical simulations of a partially mixed estuary (MacCready and Geyer, 2001) indicate that while the maximum rates of buoyancy flux occur locally during ebbs, the flood is the most productive period of vertical salt flux due to the elongation of the isohalines and a larger area over which the weaker mixing processes present on the flood can act. This is consistent with the results of Peters(1999) who found that, across the tidal cycle, floods were the most effective period for mixing in the Hudson River Estuary, 
with the exception of spring ebbs. Such a case is unlikely to be found in the Fraser River (at least during spring tide, freshet conditions) because the isohaline structure is substantially eroded during ebb tides, and the floods begin with a salt wedge that is considerably shorter in length than is present at the beginning of the ebb tides. A rebuilding of the salt wedge, fed by landward advection of dense near-bottom water, occurs throughout the flood.

\subsection{Concluding Remarks on Mixing Variability}

Diapycnal mixing plays a vital role in the dynamics of the Fraser River estuary, and is the primary mechanism responsible for the daily purge of salt from the estuarine channel during high flow, spring tide periods. The nature of the mixing climate is highly variable, both spatially and temporally. Spatial heterogeneity in mixing intensity is due primarily to variations in channel width, as described by Geyer (1985). Temporal variability is highly influenced by the strength of barotropic forcing, and initial conditions set up by prior phases of the tide. Average mixing during ebbs, primarily focused at localized channel constrictions, appears to be on the order of 2 to 6 times larger than the average mixing observed during the remainder of the tidal cycle, but mixing processes appear active and important to the system dynamics throughout the tidal cycle. 
Chapter 5

\section{Conclusions}


This thesis has presented an investigation of the highly stratified estuarine dynamics associated with the Fraser River outflow, with conclusions that are widely applicable to other energetic, shear-stratified flows. Hydrographic and bathymetric conditions in the Fraser during the summer freshet provide an ideal setting for studying the fluid dynamics specific to highly sheared and stratified systems. A turbulent Reynolds number, $\varepsilon / v N^{2}$, is often used to characterize such systems. Most previous research has been conducted in the laboratory and at relatively low energy field sites, where values of $\varepsilon / \nu N^{2}$ rarely exceed $10^{2}$. The conditions observed in the Fraser estuary can be characterized by values of $\varepsilon / v N^{2}$ on the order of $10^{4}$. Clearly, these observations from the Fraser have provided insight into the dynamics of shear-stratified flows at scales that are not reproducible in the laboratory, but can be quite common in environmental flows.

\subsection{The Dynamic Cycle of the Fraser River}

A typical tidal cycle in the Fraser River Estuary during the summer freshet can be separated into two distinct dynamical periods: a short period of several hours near the end of the largest daily ebb, characterized by a nearly steady-state salt front near the estuary mouth, and the remainder of the tidal cycle, which is characterized by the time dependent development of a salt wedge within the channel. The second period can be further dynamically segmented into flood and ebb components. Chapters 2 and 3 of this thesis focused on the steady state period of the tidal cycle, specifically addressing frontal structure and stratified turbulence seaward of the front, respectively. Chapter 4 addressed issues of variability in buoyancy flux during the salt wedge portion of the tidal cycle. 
Extending from earlier studies (e.g., Geyer, 1985; Geyer and Farmer, 1989), Chapter 4 provides an improved understanding of the temporally and spatially dependent dynamics within the channel.

The structure of the front during the steady-state period is well described by twolayer hydraulic theory, modified for three-dimensions. A Froude angle, defined as the arcsine of the inverse Froude number, was found to describe the angle of the front with respect to the streamlines of the discharging flow. In this sense, a critical Froude number of one is only observed at the front when calculated along a frame of reference perpendicular to the front. An ambient, sub-pycnocline transverse flow, driven by the response of the Strait of Georgia to the ebbing tide, provides the influx of salt and volume required to feed the mixing processes within the plume.

Turbulence, mixing and entrainment seaward of the front during the steady-state period were investigated in Chapter 3 . High rates of TKE production and buoyancy flux were observed in the first kilometer behind the front, with typical values of production on the order of $10^{-3} \mathrm{~m}^{2} \mathrm{~s}^{-3}$. The partitioning of the produced TKE into potential energy (through processes quantified by the buoyancy flux), and dissipation, is described by the flux Richardson number, which typically falls between 0.15 and 0.25 , consistent with laboratory studies performed at much lower values of $\varepsilon / \nu N^{2}$. Entrainment processes, which draw ambient fluid into the expanding plume, were also quantified, and related to the mean outflow velocity by an entrainment coefficient on the order of $10^{-3}$. This value is also consistent with the results of laboratory experiments performed under substantially less energetic conditions. 
As the steady state period wanes, the dynamics near the front are affected by a substantial pressure gradient in the ambient fluid, and the salt wedge begins its daily advance into the channel. The wedge advances some 10 to $20 \mathrm{~km}$ landward during the initial flood, persisting in the channel during the minor ebb and flood, until the system finally clears itself of salt during the dominant ebb. Observations from a time series discussed in Chapter 4 suggest that the majority of salt is removed from the channel through mixing processes, as opposed to an advective retreat of the salt wedge. The time series analysis also shows that the average rate of buoyancy flux in the channel through the tidal cycle is approximately an order of magnitude lower than observed at the mouth in Chapter 3.

The temporal evolution of the salt wedge was related to equilibrium between straining and mixing processes. Mixing may play an important role throughout the tidal cycle, but it appears to be more significant during ebb tides. Buoyancy flux rates during the flood may be of the same order as those observed during the ebb, or up to a factor of six smaller, but are likely on the order of two to three times less intense that those observed during the ebb. Geyer (1985) suggested that mixing during the ebb might be focused spatially at constrictions or other locations of potential hydraulic control. Spatial quantification of mixing processes during the ebb supported this conclusion, as described in Chapter 4.

The overall influence of mixing through the tidal cycle results in the flushing of salt from the channel several hours prior to the beginning of the dominant flood tide, providing an opportunity for the system to reset initial conditions on each tidal cycle. 
This cycle, modulated due to the fortnightly variations in tidal height, and variations in river discharge continues daily through the freshet period, which typically lasts for one to two months during the early summer.

\subsection{Contributions of the Thesis}

Specific information regarding the details of the daily tidal cycle in the Fraser River are ultimately of importance only to those residing in the Lower Delta whose lives are influenced by the Fraser and the nuances of its daily, seasonal and yearly cycles. However, the fluid dynamics observed in the Fraser River have wide applicability to flows in other estuaries and coastal systems, as well as flows in other realms, such as the deep ocean, fresh water reservoirs, and the atmosphere. To that end, the most significant contributions of the thesis can be summarized as follows.

\subsubsection{Three-Dimensional Extension of Two-Layer Hydraulic Theory}

Two-layer hydraulic theory, as presented by Armi and Farmer (1986) and Farmer and Armi (1986), is based on a channel that is uniform in the transverse direction. Most

real estuaries and straits exhibit cross-channel variability to some degree, however, no extension of two layer hydraulic theory has been developed to address the three dimensional nature of these flows. In Chapter 2, the Froude angle was presented as a means of expanding two layer hydraulic theory to three dimensions. Application of this extended theory to the data from the Fraser is consistent with the observations, in contrast to the two-dimensional theory. 
The hydraulic control principles discussed in Chapter 2 are likely relevant to all manner of hydraulic control situations, and not only to the arrested front scenario observed at the mouth of the Fraser River. Application of these principles to other regimes may allow for an increased understanding of flow through straits at many scales, from the relatively small scales observed in estuaries, to large overflows in the deep ocean. Observations of these types of flows are often confined to two-dimensions, usually focused on the deepest path through the strait. As described in Chapter 2, there must be some region within any hydraulically controlled flow that is locally twodimensional in order to anchor the control. Typically, this must occur within the deepest part of the channel, or near a channel boundary. Therefore, the apparent twodimensionality observed in many straits may only be a local effect, while unobserved three dimensional aspects of the flow may have a significant influence over the hydraulic capacity of the strait. The discussion in Chapter 2 provides a means for predicting the location and shape of the control, given three-dimensional knowledge of the strait hydrography.

\subsubsection{Development of Control Volume Method For Turbulent Flux Calculations}

The calculation procedure developed in Chapter 3 provides a means of deriving turbulent flux estimates from mean velocity and density profile measurements, which proved a robust alternative to microstructure measurements (e.g., Gargett and Moum, 1995; Peters, 1999), overturn methods (e.g., Thorpe, 1979; Osborn, 1980; Dillon, 1982), and ADCP variance techniques (Stacey et al., 1999). The key to the method's success lies in its ability to separate entrainment processes from turbulent processes. This method 
has limitations, however, and can only be successful in regions where lateral influxes are small and the turbulence signal is large.

\section{$\underline{\text { 5.2.3 Mixing Efficiencies }\left(R i_{f}\right) \text { in Energetic Shear-Stratified Flows }}$}

Although the nature of shear-stratified flows has been well studied in the laboratory (i.e., Ivey and Imberger, 1991), with flux Richardson numbers consistently observed in the vicinity of 0.2 , few field scale observations have been available from highly energetic systems, and it has been unclear if the laboratory results are representative of larger scale natural systems. The observations presented in Chapter 3 indicate that the majority of mixing in the Fraser lift-off region occurs at gradient Richardson numbers between 0.2 and 0.25 , with flux Richardson numbers between approximately 0.15 and 0.25 . As this region is characterized by values of $\varepsilon / v N^{2}$ on the order of $10^{4}$, two to three orders of magnitude higher than most laboratory studies and other field observations, this study has provided a valuable extension of the understanding of shear-stratified flows to larger energy containing scales.

\subsubsection{Simple Turbulent Closure Scheme for Pure Shear-Stratified Flows}

As noted by Imberger and Ivey (1991), there should be only two independent variables in a pure shear-stratified flow: the shear, represented by $\Delta U$, and the stratification, represented by $\Delta \rho$, or $g^{\prime}$. Based on these parameters, the turbulence and entrainment associated with the flow should be fully characterized by an entrainment coefficient and a stratified drag coefficient, as described in Chapter 3. From these two ratios, estimates of turbulent quantities can be generated as follows: 


$$
\begin{aligned}
& \overline{u^{\prime} w^{\prime}}=C_{D i}(\Delta U)^{2} \\
& w_{e}=E(\Delta U) \\
& P=\frac{C_{D i}}{R i_{g}}(\Delta U) g^{\prime} \\
& B=R i_{f} \cdot P
\end{aligned}
$$

The issue of turbulence closure has been a key issue in fluid dynamics for decades (e.g., Mellor and Yamada, 1982), with important ramifications for modeling and other predictive analyses in many diverse applications. The discussion in Chapter 3 suggested that a simple parameterization scheme for entrainment and turbulent processes within a pure shear-stratified flow, such as described in (5.1), should be relevant within regions of fully developed turbulence. The turbulence observed in the Fraser may not have been fully developed, and the evolution of turbulent scales through the lift-off zone may complicate the simplicity of the relationships in (5.1). Further complications could arise from the presence of ambient conditions that might limit plume expansion, or otherwise introduce additional constraints on the system dynamics. Nevertheless, a mean value for the entrainment coefficient identified for the lift-off zone was on the order of $10^{-3}$, with a shear-stratified drag coefficient on the order of $5 \times 10^{-4}$.

\subsubsection{Importance of Mixing Throughout the Tidal Cycle}

Observations of salt wedge structure throughout the tidal cycle in Chapter 4 suggest that mixing is more intense during ebbs, but that mixing during floods may remain a significant contributor to the mean tidal-cycle integrated buoyancy flux. This is consistent with recent findings in the Hudson (Peters, 1999) and recent numerical simulations of a partially stratified estuary by MacCready and Geyer (2001). The present 
study also validated observations by Geyer (1985) that mixing is enhanced within localized channel constrictions.

\subsection{Unresolved Issues}

Although this thesis has provided an enhanced understanding of the stratified dynamics within the Fraser River, and made significant contributions to the general understanding of hydraulic control and shear-stratified turbulence, some issues remain which may require additional investigation to adequately resolve. In Chapter 2, application of a Froude angle was found to predict the location of the arrested estuarine front at the mouth of the Fraser quite well. Further studies in controlled environments, either in the laboratory or other field experiments, may be necessary to demonstrate the broader applicability of this concept and its implications. Additionally, while it has been suggested that similar dynamics may affect hydraulic control in situations where both layers are active, three-dimensional field observations of such flows are limited.

Observations of the lateral structure of hydraulic control in such venues as Knight Inlet (Klymak and Gregg, 2001), the Straits of Gibraltar (Farmer and Armi, 1988), and the Denmark Strait Overflow (Girton et al, 2001), for example, could be useful in identifying the generality of the Froude angle concept.

In Chapter 3, the control volume method for estimating turbulent fluxes was found to be robust in the top five to six meters of the water column, due to relatively small lateral effects. Interesting comparisons could be generated through the use of microstructure profilers to provide alternative profiles of turbulent fluxes. Such measurements could 
corroborate the results obtained using the control volume method in the upper portion of the water column, and, perhaps more importantly, provide better insight into the characteristics of the turbulence deeper in the water column, at the base of the plume. Additional observations of other pure shear-stratified environmental flows are also necessary to evaluate the effectiveness of the simple closure schemes proposed in

\section{Chapter 3.}

Further investigation into the nature of the mixing processes in the Fraser River channel are necessary to address the potential for mixing mechanisms driven by secondary flows within the vicinity of Steveston Bend. Such mechanisms could contribute significantly to the total buoyancy flux budget in the channel, particularly during the ebb. These studies might incorporate similar measurement techniques to those used in the present study, but with sampling focused on the shallow areas to the inside of the bend, which would require the use of smaller vessels. The results would provide a better understanding of the mechanisms specific to mixing in the Fraser River estuary, and also allow comparisons with other regions where secondary flows appear to be important to the mixing climate, such as the Tacoma Narrows region of Puget Sound (Seim and Gregg, 1997), and San Francisco Bay (Lacy and Monismith, 2001).

Ultimately, a greater understanding of the general dynamics of the Fraser River Estuary does not preclude the region from further study. To the contrary, it provides a more controlled setting for studying specific fluid dynamic principles at large scales, and under highly energetic conditions. The Fraser River Estuary will continue to serve as an ideal natural laboratory, even as the body of knowledge about the system is increased. 


\section{Appendix: A New Stratification Parameter}

The degree of stratification maintained in an estuary is the result of a balance between two competing mechanisms (Simpson et al., 1990): the stratifying effects of velocity-induced straining, including both estuarine circulation and tidal motion, and the homogenizing effects of mixing. The concept of velocity-induced straining involves the tilting, or elongating, of isopycnals when acted upon by some sheared horizontal velocity profile. As described by Simpson et al. (1990), this action can result in a time rate of change of a stratification parameter, $\phi$, which represents the amount of energy input needed to homogenize the vertical density profile:

$$
\frac{\partial \phi}{\partial t}=\frac{g}{h} \frac{\partial \rho}{\partial x} \int_{h}^{\rho}(u-\hat{u}) z d z
$$

where $h$ represents the depth of the fluid, $\rho$ is the fluid density, $u$ is the along-channel velocity component, $\hat{u}$ is the vertically averaged along-channel velocity, and $x$ and $z$ are the along-channel and vertical coordinates, respectively. While $\phi$ can be a useful parameter for gauging stratification in estuaries, it can also be misleading, particularly in areas of intense interfacial wave activity, as its value changes depending on the vertical location of the layer interface relative to mid-depth. A non-dimensional representation of mixed layer thickness is proposed as an alternative stratification parameter:

$$
\psi=\frac{1}{2} \frac{h}{L_{50}}, \quad L_{50}=z_{75}-z_{25}
$$

where $h$ represents the water column depth, and $L_{50}$ represents the vertical distance between the $75^{\text {th }}\left(z_{75}\right)$ and $25^{\text {th }}\left(z_{25}\right)$ percentiles of the system salinity range. If both 
isohalines are not observed at a given location, the value of $L_{50}$ can be estimated based on observable salinity gradients, thus allowing values of $L_{50}$ exceeding $h$ and values of $\psi$ less than 0.5 . The dimensionless stratification parameter, $\psi$, can be interpreted as follows:

$$
\begin{array}{ll}
\psi<<1 & \text { Well mixed } \\
\psi \sim 1 & \text { Partially stratified } \\
\psi \gg>1 & \text { Highly stratified }
\end{array}
$$

In general, the effect of velocity-induced straining on this new stratification parameter can be represented as:

$$
\left(\frac{\partial \psi}{\partial t}\right)_{\text {straining }}=\left(\frac{h}{\Delta S_{\max }}\right) \frac{\partial u}{\partial z} \frac{\partial S}{\partial x} .
$$

where $\Delta S_{\max }$ represents the span of the system salinity range. Depending on the shape of the velocity profile and the structure of the isopycnals, the effect of straining can either strengthen or weaken the stratification. However, the mechanism remains the primary means of enhancing or creating stratification, and is balanced primarily by turbulent mixing.

The physical act of mixing is typically accomplished by turbulence acting against a stable density gradient. The shear production, $P$, of turbulent kinetic energy (TKE) can be expressed as:

$$
P=\overline{u^{\prime} w^{\prime}} \frac{\partial u}{\partial z}
$$

where the primed values represent turbulent fluctuations, and the overbar represents a 
mean. Some of this TKE is stored as potential energy, as salt mass is physically lifted higher in the water column, and can be quantified as buoyant production, or buoyancy flux, $B$ :

$$
B=\frac{g}{\rho} \overline{\rho^{\prime} w^{\prime}}=g \beta \overline{S^{\prime} w^{\prime}}
$$

where $\mathrm{g}$ represents the gravitational acceleration, and $\beta=\frac{1}{\rho} \frac{\partial \rho}{\partial S}=0.77 \times 10^{-3} \mathrm{psu}^{-1}$. The third major term in the steady state, one-dimensional TKE equation is the TKE dissipation term, $\varepsilon, P=B+\varepsilon$. If a flux Richardson number is defined as the ratio of shear production to buoyant production, $R_{f}=P B^{-1}$, then a mixing efficiency, equal to the ratio of buoyant production to dissipation, $E$, can be expressed as $E=R_{f} /\left(1-R_{f}\right)=B \varepsilon^{-1}$. Expressing the vertical turbulent density flux as an interfacial stress, $\tau_{i}=\rho \overline{u^{\prime} w^{\prime}}$, an expression relating interfacial stress to buoyancy flux can be derived by combining equations (A.4) and (A.5), and making use of the flux Richardson number:

$$
\tau_{i} \frac{\partial u}{\partial z}=\frac{1}{R_{f}} g \overline{\rho^{\prime} w^{\prime}}
$$

If the interfacial stress is parameterized using a drag coefficient, $C_{D}$, then:

$$
B=R_{f} C_{D} \frac{\partial u}{\partial z}|\Delta u| \Delta u
$$

Using this parameterization of the buoyancy flux, and assuming $\partial S / \partial z$ is a constant through the water column, the effect of mixing on stratification can be expressed as: 


$$
\left(\frac{\partial \psi}{\partial t}\right)_{\text {mixing }}=\frac{12 R_{f} C_{D} \frac{\partial u}{\partial z}|\Delta u| \Delta u}{g \beta(\Delta S)_{\max } h}
$$

This formulation, particularly the parameterization of vertical turbulent density flux as interfacial stress, and the resulting equation (A.6), should be effective so long as mixing is enabled. In situations where the $R i_{g}$ is not less than $1 / 4$, existing interfacial stress may not be sufficient to initiate mixing, and any observed buoyancy flux may be the result of other mixing mechanisms (i.e., not shear induced mixing). In these cases the formulation expressed in (A.8) would be invalid.

In addition to the local processes of straining and mixing, the local stratification can also be significantly affected by the advection of stratification into the local region, which was set and maintained by non-local processes. The impact of advection on local stratification can be expressed as:

$$
\left(\frac{\partial \psi}{\partial t}\right)_{\text {advection }}=u \frac{\partial \psi}{\partial x}
$$




\section{Bibliography}

Adams E.E., and K.D. Stolzenbach, 1977: Analysis of a buoyant surface discharge over a shallow sloping bottom. Proceedings of the XVII International Association of Hydraulic Research (IAHR) Congress, Baden Baden, 363-370.

Armi, L., 1986: The Hydraulics of two flowing layers with different densities. J. Fluid Mechanics, vol. 163, 27-58.

Armi, L., and D.M. Farmer, 1986: Maximal two-layer exchange through a contraction with barotropic net flow. J. Fluid Mechanics, vol. 164, 27-51.

Atkinson, J.F., 1993: Detachment of buoyant surface jets discharged on slope. J. of Hydraulic Engineering, ASCE, vol. 119(8), 878-894.

Baines, P.G., 1995: Topographic Effects in Stratified Flows. Cambridge University Press, UK, 88-90.

Balmforth, N.J., S.G. Llewellyn Smith, and W.R. Young, 1998: Dynamics of interfaces and layers in a stratified turbulent fluid. J. Fluid Mechanics, vol. 355, 329-358.

Barry, M.E., G.N. Ivey, K.B. Winters, and J. Imberger, 2001: Measurements of diapycnal diffusivities in stratified fluids. J. Fluid Mechanics, vol. 442, 267-291.

Bates, C.C., 1953: Rational theory of delta formation. Bulletin of the American Association of Petroleum Geologists, vol. 37(9), 2119-2161.

Baumert H. and H. Peters, 2000: Second-moment closures and length scales for weakly stratified turbulent shear flows. Journal of Geophysical Research, vol. 105(C3), 6453-6468.

Benjamin, T.B., 1968: Gravity currents and related phenomena. J. Fluid Mechanics, vol. $31(2), 209-248$.

Bondar, C., 1970: Considerations theoriques sur la dispersion d'un courant liquide de densite reduite et a niveau libre, dans un basin contenant un liquide d'une plus grande densite, Symposium on the Hydrology of Deltas, UNESCO, vol. 11, 246256. 
Bowden, K.F., 1967: Circulation and diffusion, in Estuaries, G.H. Lauff ed., American Association for the Advancement of Science, 15-36.

Caspers, H., 1967: Estuaries: Analysis of definitions and biological considerations, in Estuaries, G.H. Lauff ed., American Association for the Advancement of Science, 6-8.

Chapman, A.J., and W.F. Walker, 1971. Introductory Gas Dynamics. Holt, Rinehart and Winston, Inc., New York, 369-391.

Christodoulou, G.C., 1986: Interfacial mixing in stratified flows. Journal of Hydraulic Research, vol. 24(2), 77-92.

Crawford, W.R., 1986: A comparison of length scales and decay times of turbulence in stably stratified flows. J. Physical Oceanography, vol. 16, 1847-1854.

Crean, P.B., T.S. Murty, and J.A. Stronach, 1988: Mathematical Modelling of Tides and Estuarine Circulation. Springer-Verlag, Berlin, 30.

Dallimore, C.J., J. Imberger, and T. Ishikawa, 2001: Entrainment and turbulence in saline underflow in Lake Ogawara. Journal of Hydraluic Engineering, ASCE, vol. 127(11), 937-948.

Dalziel, S.B., 1991: Two-layer hydraulics: a functional approach. J. Fluid Mechanics, vol. 223, 135-163.

Dillon, T.M., 1982: Vertical overturns: a comparison of Thorpe and Ozmidov length scales. Journal of Geophysical Research, vol. 87(C12), 9601-9613.

Ellison, T.H. and J.S. Turner, 1959: Turbulent entrainment in stratified flows. J. Fluid Mechanics, vol. 6, 423-448.

Etemad-Shahidi, A, and J. Imberger, 2001: Anatomy of turbulence in thermally stratified lakes. Limnology and Oceanography, vol. 46(5), 1158-1170.

Farmer, D., and L. Armi, 1999: Stratified flow over topography: the role of small-scale entrainment and mixing in flow establishment. Proc. R. Soc. London A, vol. 455, 3221-3258.

Farmer, D.M., and L. Armi, 1988: The flow of Mediterranean water through the Strait of Gibraltar, and Armi, L. and D.M. Farmer, 1988: The flow of Atlantic water through the Strait of Gibraltar. Progress in Oceanography, vol. 21, 1-105.

Farmer, D.M., and L. Armi, 1986: Maximal two-layer exchange over a sill and through the combination of a sill and contraction with barotropic flow. J. Fluid Mechanics, vol. 164, 53-76. 
Ferron, B, H. Mercier, K. Speer, A. Gargett, and K. Polzin, 1998: Mixing in the Romanche Fracture Zone. J. Physical Oceanography. vol. 28(10), 1929-1945.

Fong, D.A., 1998: Dynamics of freshwater plumes: observations and numerical modeling of the wind-forced response and alongshore freshwater transport. Ph.D. Thesis, MIT/WHOI Joint Program in Oceanography, Woods Hole, Massachusetts.

Gargett, A.E. and J.N. Moum, 1995: Mixing efficiencies in turbulent tidal fronts: results from direct and indirect measurements of density flux. J. Physical Oceanography, vol. 25, 2583-2608.

Garvine, R.W., 1974: Physical features of the Connecticut River outflow during high discharge. Journal of Geophysical Research, vol. 79(6), 831-846.

Garvine, R.W., 1982: A steady state model for buoyant surface plume hydrodynamics in coastal waters. Tellus, vol. 34, 293-306.

Gerdes, F., C. Garrett, and D. Farmer, 2002: On internal hydraulics with entrainment. $J$. Physical Oceanography. vol. 32, 1106-1111.

Geyer W.R., 1993: Three-dimensional tidal flow around headlands. Journal of Geophysical Research, vol. 98(C1), 955-966.

Geyer, W.R. and G.A. Cannon, 1982: Sill processes related to deep water renewal in a fjord. Journal of Geophysical Research, vol. 87, 7985-7996.

Geyer, W.R. and G.C. Kineke, 1995: Observations of currents and water properties in the Amazon frontal zone. Journal of Geophysical Research, vol. 100(C2), 23212339.

Geyer, W.R., 1985: The Time-Dependent Dynamics of a Salt Wedge. Ph.D. Thesis, University of Washington, Seattle, Washington.

Geyer, W.R., 1988: The advance of a salt wedge front: Observations and dynamical model. Physical Processes in Estuaries, Job Dronkers and Wim van Leussen, eds. Springer-Verlag, Berlin,181-195.

Geyer, W.R., and D.M. Farmer, 1989: Tide-induced variation of the dynamics of a salt wedge estuary. J. Physical Oceanography. vol. 19, 1060-1072.

Geyer, W.R., and J.D. Smith, 1987: Shear instability in a highly stratified estuary. $J$. Physical Oceanography, vol. 17(10), 1668-1679.

Geyer, W.R., R.P. Signell and G.C. Kineke,1998: Lateral trapping of sediment in a partially mixed estuary. 8th International Biennial Conference on Physics of 
Estuaries and Coastal Seas, 1996, Dronkers and Scheffers (eds), Balkema, Rotterdam, pp 115-124.

Gibson, C., 1998: Intermittency of internal wave shear and turbulence dissipation. In Physical Processes in Lakes and Oceans, J. Imberger (ed), AGU, Washington D.C., 363.

Girton, J.B., T.B. Sanford, and R.H. Käse, 2001: Synoptic sections of the Denmark Strait overflow. Geophys. Res. Letters, vol. 28, 1619-1622.

Grant, H.L., R.W. Stewart, and A. Moilliet, 1962: Turbulence spectra from a tidal channel. J. Fluid Mechanics, vol. 12, 241-268.

Gregg, M.C., 1987: Diapycnal mixing in the thermocline: A review. J. Geophysical Research, vol. 92, 5249-5286.

Gregg, M.C., 1989: Scaling turbulent dissipation in the thermocline. J. Geophysical Research, vol. 94 (C7), 9686-9698.

Henderson, F.M., 1966: Open Channel Flow, Macmillan, New York.

Hickey, B.M., L.F. Pietrafesa, D.A. Jay, and W.C. Boicourt, 1998: The Columbia River plume study: subtidal variability in the velocity and salinity fields. Journal of Geophysical Research, vol. 103(C5), 10339-10368.

Howard, L.N., 1961: Note on a paper by J.W. Miles. J. Fluid Mechanics., vol. 10, 509512.

Huzzey, L.M., 1982: The dynamics of a bathymetrically arrested estuarine front. Estuarine, Coastal and Shelf Science, vol. 15, 537-552.

Imberger, J. and G.N. Ivey, 1991: On the nature of turbulence in a stratified fluid. Part II: application to lakes. J. Physical Oceanography, vol. 21, 659-680.

Ippen, A.T., 1936: An Analytical and Experimental Study of High Velocity Flow in Curved Sections of Open Channels. Ph.D. Thesis, California Inst. Tech., Pasadena.

Ivey, G.N. and J. Imberger, 1991: On the nature of turbulence in a stratified fluid. Part I: the energetics of mixing. J. Physical Oceanography, vol. 21, 650-658.

Jirka, G.H., and A. Masamitsu, 1987: Density currents or density wedges: boundarylayer influence and control methods. J. Fluid Mechanics, vol. 177, 187-206.

Jirka, G.H., E.E. Adams, and K.D.Stolzenbach, 1981: Buoyant surface jets. Journal of the Hydraulics Division, A.S.C.E., vol. 107, 1467-1487. 
Kashiwamura , M. and S. Yoshida, 1967: Outflow pattern of fresh water issued from a river mouth. Coastal Engineering in Japan, vol. 10, 109-115.

Kashiwamura, M. and S. Yoshida, 1978: Outflow dynamics at a river mouth. Proceedings of the $16^{\text {th }}$ Coastal Engineering Conference, A.S.C.E., 2925-2944.

Klymak, J.M., and M.C. Gregg, 2001: Three-dimensional nature of flow near a sill. $J$. Geophysical Research, vol. 106(C10), 22,295-22,311.

Lacy, J.R., and S.G. Monismith, 2001: Secondary currents in a curved, stratified, estuarine channel. J. Geophys. Res., vol. 106(C12), 31283-31302.

Ledwell, J.R., A.J. Watson and C.S. Law, 1993: Evidence for slow mixing across the pycnocline from an open-ocean tracer-release experiment. Nature, vol. 364, 701702.

Lentz, S.J., 2001: The influence of stratification on the wind-driven cross-shelf circulation over the North Carolina shelf. J. Physical Oceanography, vol. 31, 2749-2760.

Liggett, J.A., 1994: Fluid Mechanics. McGraw-Hill, New York, 319-326.

Linden, P.F., 1979: Mixing in stratified fluids. Geophy. Astrophy. Fluid Dynamics, vol. $13,3-23$.

MacCready P. and W.R. Geyer, 2001: Estuarine salt flux through an isohaline surface, Journal of Geophysical Research, vol. 106(C6), 11629-11637.

McDougall, T.J., 1984: The relative roles of diapycnal and isopycnal mixing on subsurface water mass conversion. J. Physical Oceanography, vol. 14, 15771589.

Mellor, G.L., and T. Yamada, 1982: Development of a turbulence closure model for geophysical fluid problems. Reviews of Geophysics and Space Physics, vol. 20(4), 851-875.

Miles, J.W., 1961: On the stability of heterogeneous shear flow. J. Fluid Mechanics, vol. 10, 496-508.

Morton, B.R., G. Taylor, and J.S. Turner, 1956: Turbulent gravitational convection from maintained and instantaneous sources. Proc., Royal Soc., London A, vol. 234, 1 23.

Moum, J.N., 1996: Efficiency of mixing in the main thermocline. J. Geophys. Res., vol. $101,12057-12069$. 
Nepf, H.M. and W.R. Geyer, 1996: Intratidal variations in stratification and mixing in the Hudson estuary. Journal of Geophysical Research, vol. 101(C5), 1207912086.

Osborn, T.R., 1980: Estimates of the local rate of vertical diffusion from dissipation measurements, J. Physical Oceanography, vol. 10, 83-89.

Park, C., J. Kim, and S Chang, 1993: The characteristics on the mixing of freshwater in Suyoung Bay. Bulletin of Korean Fisheries Society, vol. 26(4), 353-362.

Partch, E.N. and J.D. Smith, 1978: Time dependent mixing in a salt wedge estuary. Estuarine and Coastal Marine Science, vol. 6, 3-19.

Pawlak, G. and L. Armi, 2000: Mixing and entrainment in developing stratified currents. J. Fluid Mechanics, vol. 424, 45-73.

Peters, H., 1999: Spatial and temporal variability of turbulent mixing in an estuary. Journal of Marine Research, vol. 57(6), 805-845.

Peters, H., M.C. Gregg, and J.M. Toole, 1988: On the parameterization of equatorial turbulence. Journal of Geophysical Research, vol. 93(C2), 1199-1218.

Pritchard, D.W., 1952: Salinity distribution and circulation in the Chesapeake Bay estuarine system. J. Marine Research, vol. 11, 106-123.

Pritchard, D.W., 1954: A study of the salt balance in a coastal plain estuary. J. Marine Research, vol. 13, 133-144.

Pritchard, D.W., 1956: The dynamic structure of a coastal plain estuary. J. Marine Research, vol. 15, 33-42.

Schijf, J.B. and J.C. Schonfeld, 1953: Theoretical considerations on the motion of salt and fresh water. Proceedings of the Minnesota International Hydraulics Convention, $5^{\text {th }}$ Congress I.A.H.R., 321-333

Seim, H.E. and M.C. Gregg, 1997: The importance of aspiration and channel curvature in producing strong vertical mixing over a sill. Journal of Geophysical Research, vol. 102(C2), 3451-3472.

Simpson, J.H., J. Brown, J. Matthews, and G. Allen, 1990: Tidal straining, density currents, and stirring in the control of estuarine stratification. Estuaries, vol. 13(2), 125-132.

Smyth, W.D. and J.N. Moum, 2000: Length scales of turbulence in stably stratified mixing layers. Physics of Fluids, vol. 12(6), 1327-1342. 
Sommerfield, C.K. and C.A. Nittrouer, 1999: Modern accumulation rates and a sediment budget for the Eel shelf: A flood-dominated depositional environment. Marine Geology vol. 154, 227-241.

Stacey, M.T., S.G. Monismith, J.R. Burau, 1999: Measurements of Reynolds stress profiles in tidal flows. Journal of Geophysical Research, vol.104(C5), 1093310949.

Stillinger, D.C., K.N. Helland, and C.W. Van Atta, 1982: Experiments on the transition of homogeneous turbulence to internal waves in a stratified fluid. J. Fluid Mechanics, vol. 131, 91-122.

Stommel, H., 1953: Computation of pollution in a vertically mixed estuary. Sewage and Industrial Wastes, vol. 25, 1065-1071.

Stommel, H., and H.G. Farmer, 1952: Abrupt change in width in two-layer open channel flow. J. Marine Research, vol. 11, 205-214.

Takano, K., 1954: On the velocity distribution off the mouth of a river. J. Oceanographic Society of Japan, vol. 10(2), 60-64.

Takano, K., 1955: A complementary note on the diffusion of the seaward flow off the mouth of a river. J. Oceanographic Society of Japan, vol. 11(4), 1-3.

Tennekes, H. and J.L. Lumley, 1972: A First Course in Turbulence. MIT Press, Cambridge, Massachusetts.

Thorpe S.A., 1977: Turbulence and mixing in a Scottish loch. Philos. Trans. Roy. Soc. London A, vol. 286, 125-181.

Thorpe, S.A., 1971: Experiments on the instability of stratified shear flows: miscible fluids. J. Fluid Mechanics, vol. 46, 299-319.

Thorpe, S.A., 1973: Experiments on instability and turbulence in a stratified shear flow. J. Fluid Mechanics, vol. 61, part 4, 731-751.

Wesson, J. C., and M. C. Gregg, 1994: Mixing at Camarinal Sill in the Strait of Gibraltar. Journal of Geophysical Research, vol. 99, 9847-9878.

Wijesekera, H. W., and T. M. Dillon, 1997: Shannon entropy as an indicator of age for turbulent overturns in the oceanic thermocline. Journal of Geophysical Research, vol. 102, 3279-3291.

Wijesekera, H.W., T.M. Dillon, and L. Padman, 1993: Some statistical and dynamical properties of turbulence in the oceanic pycnocline. Journal of Geophysical Research, vol. 98, 22665-22679. 
Wright, L.D. and J.M. Coleman, 1971: Effluent expansion and interfacial mixing in the presence of a salt wedge, Mississippi River Delta. J. Geophysical Research, vol. $36,8649-8661$.

Yankovsky, A.E. and D.C. Chapman, 1997: A simple theory for the fate of buoyant coastal discharges, J. Physical Oceanography, vol. 27, 1386-1401,

Yin, K., P.J. Harrison, S. Pond, and R.J. Beamish, 1995: Entrainment of nitrate in the Fraser River estuary and its biological implications. I. Effects of the salt wedge. Estuarine, Coastal and Shelf Science, vol. 40, 545-558. 